Florida International University FIU Digital Commons

$5-12-2017$

\title{
Multinucleon Short-range Correlation Model for Nuclear Spectral Functions
}

Oswaldo Artiles

Florida International University, oarti001@fiu.edu

DOI: $10.25148 /$ etd.FIDC001903

Follow this and additional works at: https://digitalcommons.fiu.edu/etd

Part of the Nuclear Commons

\section{Recommended Citation}

Artiles, Oswaldo, "Multinucleon Short-range Correlation Model for Nuclear Spectral Functions" (2017). FIU Electronic Theses and Dissertations. 3384.

https://digitalcommons.fiu.edu/etd/3384

This work is brought to you for free and open access by the University Graduate School at FIU Digital Commons. It has been accepted for inclusion in FIU Electronic Theses and Dissertations by an authorized administrator of FIU Digital Commons. For more information, please contact dcc@fiu.edu. 


\section{FLORIDA INTERNATIONAL UNIVERSITY}

Miami, Florida

MULTINUCLEON SHORT-RANGE CORRELATION MODEL FOR NUCLEAR SPECTRAL FUNCTIONS

A dissertation submitted in partial fulfillment of the

requirements of the degree of

DOCTOR OF PHILOSOPHY

in

PHYSICS

by

Oswaldo Artiles

2017 
To: Dean Michael R. Heithaus

College of Arts, Sciences and Education

This dissertation, written by Oswaldo Artiles, and entitled Multinucleon Short-range Correlation Model for Nuclear Spectral Functions, having been approved in respect to style and intellectual content, is referred to you for judgment.

We have read this dissertation and recommend that it be approved.

Oren W. Maxwell

Werner Boeglin

Gueo Grantcharov

Misak M. Sargsian, Major Professor

Date of Defense: May 12, 2017

The dissertation of Oswaldo Artiles is approved.

Dean Michael R. Heithaus
College of Arts, Sciences and Education

Andrés G. Gil

Vice President for Research and Economic Development and Dean of the University Graduate School

Florida International University, 2017 


\section{DEDICATION}

This dissertation is dedicated to Rita Elisa and Ana Rita, my grandmother and my mother, who gave me love and cared for me everyday of my childhood, and who taught me the values of honesty, respect for life, and love for hard work, values which have guided me during my entire life. 


\section{ACKNOWLEDGMENTS}

I would like to acknowledge all the teachers and professors that help me and taught me during all years of my life as a student.

I am indebted to the members of my dissertation committee: Oren W. Maxwell, Werner Boeglin, and Gueo Grantcharov, who helped me during all my research. My special acknowledgment to my adviser, Misak Sargsian, who taught me a lot of physics and was always patient with my ignorance.

I wish to acknowledge the graduate program directors, Brian Raue, Rajamani Narayanan, and Jorge Rodriguez, as well the administrative staff of the the FIU physics department, specially Elizabeth Bergano-Smith, Omar Tolbert, Maria Martinez, John Omara, Ofelia Adan-Fernandez, and Robert Brown who support me with all their administrative and technical expertise.

I also thank Frank Vera, Dhiraj Maheswari, and Christopher Leon, my fellow PhD students of the group of nuclear theoretical physics, for the very helpful physics and mathematics discussions that I have had with them.

Finally, my greatest debt to my wife Ivia, for giving me love and for taking care of me with total dedication, and to my daughters Livia and Claudia and all my grandchildren: Mauricio, Ana, Isabella e Ignacio, for all their love and for giving me the most beautiful reasons to live.

This research would not have been possible without financial support. I would like to acknowledge the Department of Energy for providing the grant that supported my research assistantship. 


\section{ABSTRACT OF THE DISSERTATION \\ MULTINUCLEON SHORT-RANGE CORRELATION MODEL FOR NUCLEAR SPECTRAL FUNCTIONS \\ by}

Oswaldo Artiles

Florida International University, 2017

Miami, Florida

Professor Misak M. Sargsian, Major Professor

The main goal of the research presented in my dissertation was to develop a theoretical model for relativistic nuclear spectral functions at high missing momenta and removal energies based on the multi-nucleon short-range correlation (SRC) model. The nuclear spectral functions are necessary for the description of high energy nuclear processes currently being studied at different labs such as JLAB, LHC and FNAL.

The model followed the effective Feynman diagrammatic approach in order to account for the relativistic effects important in the SRC domain. In addition to the two-nucleon (2N) SRC with center of mass motion contribution, the contribution of the three-nucleon SRCs to the spectral functions was also derived. The latter was modeled based on the assumption that the 3N SRCs are a product of two sequential short range nucleon-nucleon (NN) interactions.

The nuclear spectral functions models were derived from two theoretical frameworks for evaluating covariant Feynman diagrams: In the first, referred to as the virtual nucleon approximation, the Feynman diagrams were reduced to the time ordered non-covariant diagrams by evaluating the nucleon spectators in the SRC at their positive energy poles, neglecting explicitly the contribution from vacuum diagrams. In the second approach, referred to as the lightfront approximation, the boost invariant nuclear spectral function was formulated in the lightfront reference frame in which case the vacuum diagrams are kinematically suppressed and the bound nucleon is described by its light-front variables such as momentum fraction, transverse momentum and invariant mass. 
On the basis of the derived nuclear spectral functions, the corresponding computational models were developed from which the numerical estimates of the SRC spectral functions, the SRC momentum distributions, and the SRC density matrices were obtained. 


\section{TABLE OF CONTENTS}

CHAPTER

PAGE

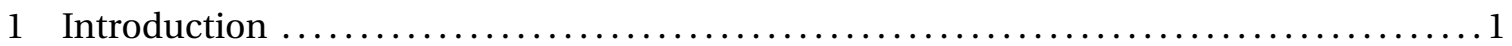

1.1 Lepton-nucleus inclusive scattering cross section and nuclear spectral function ...5

1.2 The Nuclear EuropeanMuon Collaboration (EMC) Effect $\ldots \ldots \ldots \ldots \ldots \ldots \ldots \ldots . \ldots$

1.3 Multinucleon short-range correlations in nuclei $\ldots \ldots \ldots \ldots \ldots \ldots \ldots \ldots \ldots \ldots \ldots \ldots \ldots \ldots$

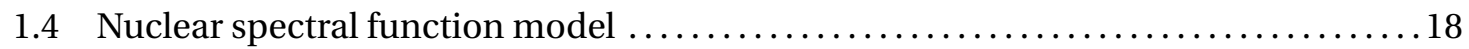

2 Multinucleon short-range correlation model for nuclear spectral functions: Theoretical

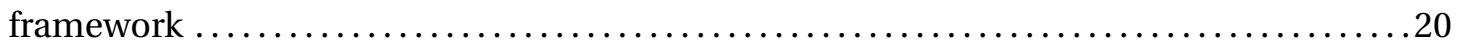

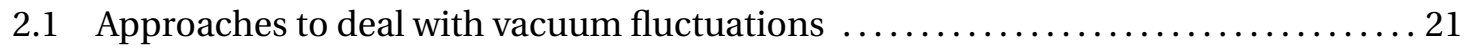

2.2 Diagrammatic approach for modeling nuclear spectral functions $\ldots \ldots \ldots \ldots \ldots \ldots 26$

2.3 Nuclear spectral function in virtual nucleon approximation $\ldots \ldots \ldots \ldots \ldots \ldots \ldots . \ldots . \ldots . .31$

2.4 Nuclear spectral function in light-front approximation $\ldots \ldots \ldots \ldots \ldots \ldots \ldots \ldots \ldots . \ldots . \ldots . \ldots 4$

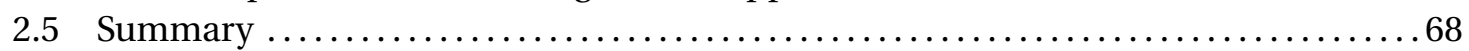

3 Multinucleon short-range correlation model for nuclear spectral functions: Models for

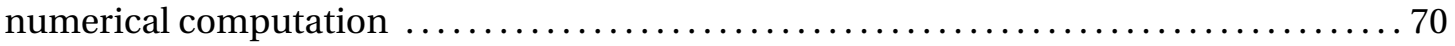

3.1 Computational models for SRC nuclear spectral function in virtual nucleon

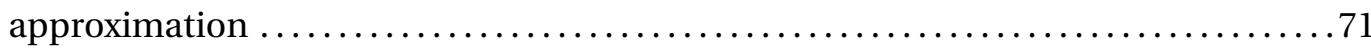

3.2 Computational models for SRC nuclear spectral function in light-front

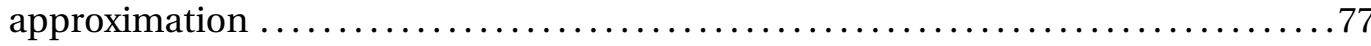

3.3 Computational models for SRC nuclear spectral function in nonrelativistic

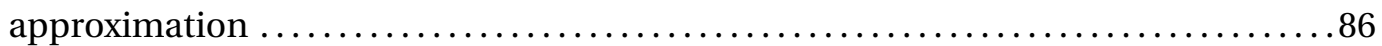

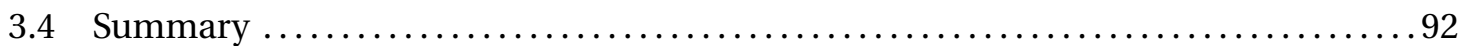

4 Numerical estimates of nuclear spectral functions, density matrices and momentum

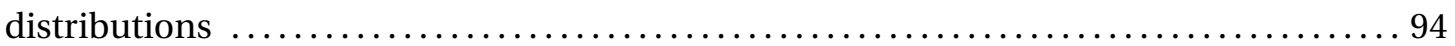

4.1 Parameters for numerical estimates of the nuclear spectral functions ...........95

4.2 Momentum distribution and light-front density matrix for deuteron $\ldots \ldots \ldots \ldots . .97$

4.3 Numerical estimates for SRC nuclear spectral function in virtual nucleon

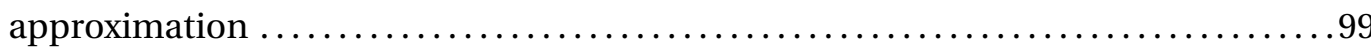

4.4 Numerical estimates for SRC nuclear spectral function in light-front

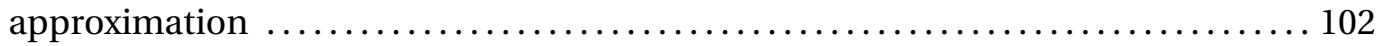

4.5 Numerical estimates for SRC density matrices in light-front approximation ..... 105

4.6 Numerical estimates for SRC momentum distribution in virtual nucleon

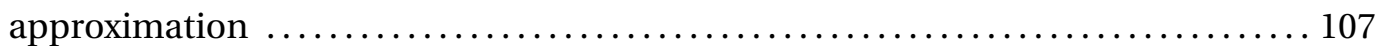

4.7 Parametrization of SRC and mean field momentum distribution $\ldots \ldots \ldots \ldots \ldots \ldots$

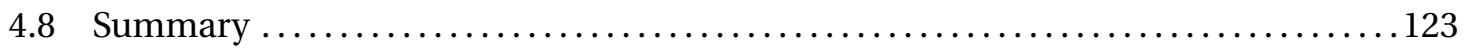

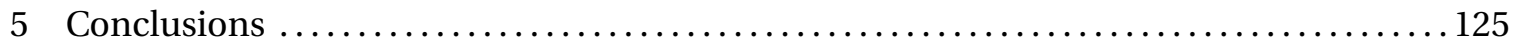

5.1 Application of the multi-nucleon short range correlation model for the nuclear

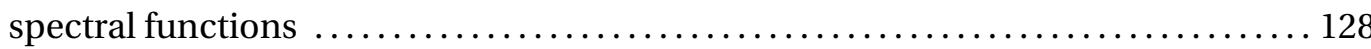




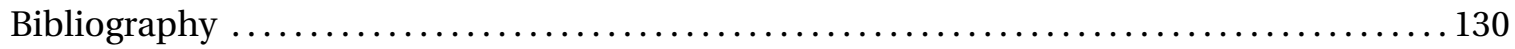

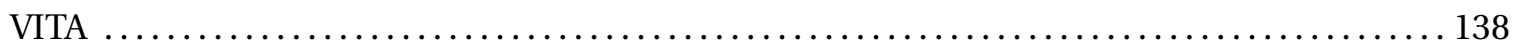




\section{LIST OF TABLES}

TABLE

PAGE

4.1 2N SRC parameters for light and heavy Nuclei $\left(\mathrm{k}_{F}\right.$ in $\left.\mathrm{GeV}\right) \ldots \ldots \ldots \ldots \ldots \ldots \ldots$

$4.23 \mathrm{~N}$ SRC parameters for light and heavy nuclei $\ldots \ldots \ldots \ldots \ldots \ldots \ldots \ldots \ldots \ldots \ldots \ldots$

4.3 Parameters for the non-relativistic mean-field momentum distribution for light

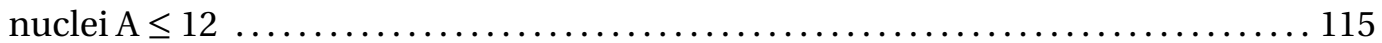

4.4 Parameters for the non-relativistic mean-field momentum distribution for heavy

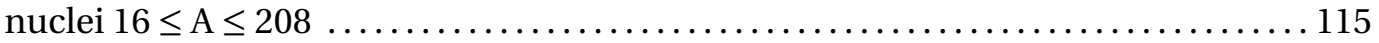

4.5 Parameters for the SRC momentum distribution in the nonrelativistic limit of the VNA

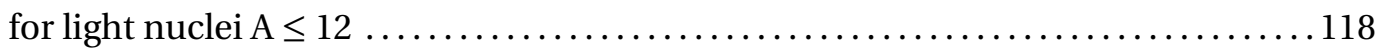

4.6 Parameters for the SRC momentum distribution in the nonrelativistic limit of the VNA

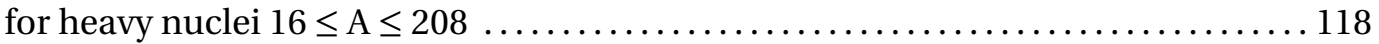

4.7 Parameters for the mean-field momentum distribution in VNA for light nuclei $\mathrm{A} \leq 12$

4.8 Parameters for the mean-field momentum distribution in VNA for heavy nuclei

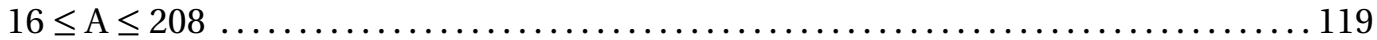

4.9 Parameters for the SRC momentum distribution in VNA for light nuclei A $\leq 12 \ldots 120$

4.10 Parameters for the SRC momentum distribution in VNA for heavy nuclei $16 \leq \mathrm{A} \leq 208$ 


\section{LIST OF FIGURES}

FIGURE

PAGE

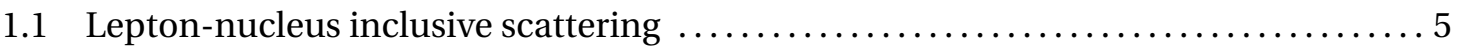

1.2 Lepton-nucleus deep inelastic inclusive scattering regions $\ldots \ldots \ldots \ldots \ldots \ldots \ldots \ldots$

1.3 Theoretical predictions for the Fermi motion corrections of the bound nucleon structure functions $F_{2}^{N}$ for iron. Figure from [38] $\ldots \ldots \ldots \ldots \ldots \ldots \ldots \ldots \ldots \ldots \ldots$

1.4 EMC experimental results for the ratio of the structure functions of iron nucleus and deuterium $\left(F_{2}^{N}(F e) / F_{2}^{N}(D)\right)$. Figure from $[38] \ldots \ldots \ldots \ldots \ldots \ldots \ldots \ldots \ldots \ldots \ldots \ldots$

1.5 Carbon EMC ratios for the highest $Q^{2}$ settings $\left(Q^{2}\right.$ quoted at $\left.\mathrm{x}=0.75\right)$. Uncertainties are the combined statistical and point-to-point systematic. The solid curve is the SLAC fit to the Carbon EMC ratio. Figure from [91] ...................... 12

$1.62 \mathrm{~N}$ and $3 \mathrm{~N}$ scaling regions for nucleus $\mathrm{A}$. Figure from Ref. [84] $\ldots \ldots \ldots \ldots \ldots \ldots \ldots$

1.7 The EMC slopes versus the SRC scale factors. Figure from Ref. [97] . ............ 14

$1.82 \mathrm{~N}$ and $3 \mathrm{~N}$ SRC models for nuclear spectral functions $\ldots \ldots \ldots \ldots \ldots \ldots \ldots \ldots$

2.1 Representation of the covariant Feynman amplitude through the sum of the time ordered amplitudes. Panel (b) corresponds to the scenario in which first, the bound nucleon is resolved in the nucleus which interacts with the incoming probe $h$. In panel (c), initially, the incoming probe produces a $\bar{N} N$, anti-nucleon and nucleon pair, with subsequent absorption of the $\bar{N}$ anti-nucleon in the nucleus. .. 22

2.2 Expansion of the nuclear spectral function into the contributions of the mean field (a), the $2 \mathrm{~N}$ (b) and the $3 \mathrm{~N}$ (c) SRCs. For each case the initial nuclear transition vertices are different, corresponding to transition of $A \rightarrow N, A-1 ; A \rightarrow$ $N N, A-1$ and $A \rightarrow N N N, A-3$ for the mean field, the $2 \mathrm{~N}$ and the $3 \mathrm{~N}$ SRCs respectively. The NN (b) and NNN (c) labels identify the $2 \mathrm{~N}$ and the $3 \mathrm{~N}$ SRCs with effective $\mathrm{NN}$ and $\mathrm{NNN}$ vertices elaborated in the text $\ldots \ldots \ldots \ldots \ldots \ldots \ldots \ldots \ldots 27$

2.3 Diagram corresponding to the 3N SRC contribution to the nuclear spectral func-

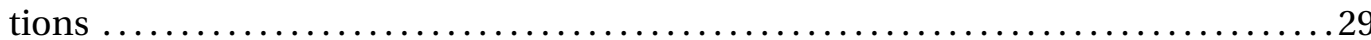

4.1 The momentum distribution of deuteron (s-wave: dot-dash, d-wave:dash) $\ldots . . .98$

4.2 Upper graph: Deuteron momentum distribution. Lower graph: Deuteron light-

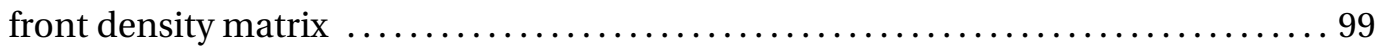

4.3 Nuclear spectral function for ${ }^{9} \mathrm{Be}$ in the nonrelativistic limit of the VNA $\ldots \ldots \ldots 100$ 
4.4 Nuclear spectral function for ${ }^{56} \mathrm{Fe}$ in the nonrelativistic limit of the VNA $\ldots \ldots \ldots 101$

4.5 Nuclear spectral function, with constant values of momentum for ${ }^{3} \mathrm{He}$ and ${ }^{9} \mathrm{Be}$, in VNA and in the nonrelativistic limit of the VNA ...................... 102

4.6 Nuclear spectral function for ${ }^{9} \mathrm{Be}$ in light-front approximation, for $\tilde{M}_{N}^{2}=0.5 \mathrm{GeV}^{2} 103$

4.7 Nuclear spectral function for ${ }^{197} \mathrm{Au}$ in light-front approximation, for $\tilde{M}_{N}^{2}=0.2 \mathrm{GeV}^{2}$

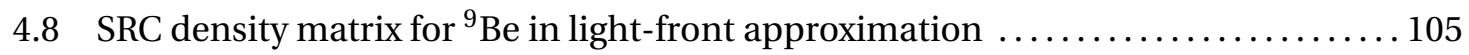

4.9 SRC density matrix for ${ }^{197} \mathrm{Au}$ in light-front approximation $\ldots \ldots \ldots \ldots \ldots \ldots \ldots \ldots$

4.10 SRC momentum distribution for proton in ${ }^{3} \mathrm{He}$, in VNA and in the nonrelativistic limit of the VNA, the predictions in the nonrelativistic limit of the VNA are compa-

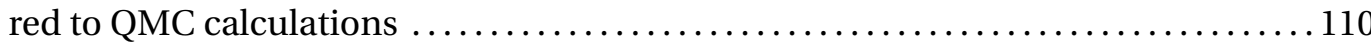

4.11 SRC momentum distribution for ${ }^{8} \mathrm{He}$ in the nonrelativistic limit of the VNA compa-

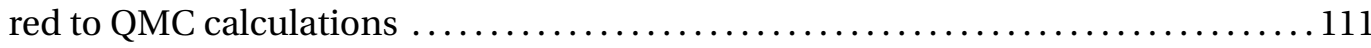

4.12 SRC momentum distribution for neutron in ${ }^{9} \mathrm{Be}$, in VNA and in the nonrelativistic limit of the VNA, the predictions in the nonrelativistic limit of the VNA are compared to QMC calculations ......................................... 112

4.13 SRC momentum distribution for ${ }^{56} \mathrm{Fe}$ in the nonrelativistic limit of the VNA compared to 2N SRC momentum distribution for proton in Ciofi/Simula [52] ........ 113

4.14 SRC momentum distribution for ${ }^{208} \mathrm{~Pb}$ in VNA compared to predictions in the non-

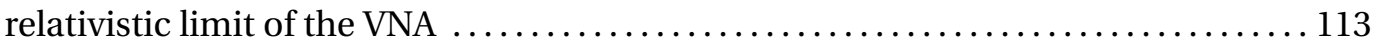

4.15 Momentum distribution for light nuclei calculated with the SRC nuclear spectral functions in the nonrelativistic limit of the VNA, compared to the curve fitting obtained with the mean field parameters in Table 4.3 and SRC parameters in Table

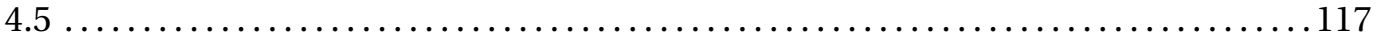

4.16 Momentum distribution for heavy nuclei calculated with the SRC nuclear spectral functions in the nonrelativistic limit of the VNA, compared to the curve fitting obtained with the mean field parameters in Table 4.4 and SRC parameters in Table

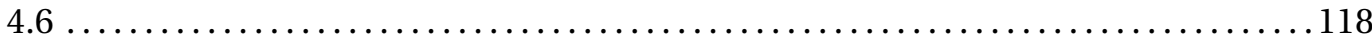

4.17 Momentum distribution for light nuclei calculated with the SRC nuclear spectral functions for VNA, compared to the curve fitting obtained with the mean field parameters in Table 4.7 and SRC parameters in Table 4.9 
4.18 Momentum distribution for heavy nuclei calculated with the SRC nuclear spectral functions for VNA, compared to the curve fitting obtained with the mean field parameters in Table 4.8 and SRC parameters in Table $4.10 \ldots \ldots \ldots \ldots \ldots \ldots . \ldots . \ldots . \ldots . \ldots 23$ 


\section{CHAPTER 1}

\section{Introduction}

Scattering experiments have been the most important experimental tools to reveal the structure of visible matter, specially the structure of atoms and nucleus. In 1911, Rutherford, through scattering experiments of alpha particles off atomic targets, discovered the atomic nucleus [1]. Experiments of disintegration of nitrogen nuclei in 1919, led Rutherford to conclude that the nucleus was a composite system of particles held together by a strong force [2]. Rutherford also gave the name of proton to the hydrogen nucleus, and predicted the neutron in 1920 [3]. The prediction of Rutherford was confirmed by Chadwick [5] and Curie-Joliot [6] who, independently, showed the existence of the neutron in 1932. The atomic nucleus has since been considered a system composed of strong interacting nucleons ${ }^{1}[7]$, held together by strong shortrange forces, of a different nature than those of the electromagnetic and gravitational forces. Since the atomic nucleus is the basic component of all the visible matter in the universe, a continuous and very important experimental and theoretical efforts have been ongoing since 1932 to understand and explain the nuclear and the nucleon structures at the fundamental level.

In 1935, Yukawa described the interaction of nucleons in the nucleus by means of a field of force associated with a particle or a quantum which was a carrier of this interaction [8]. Two particles, the pion+ and the pion-, were discovered in 1947 [9], and the neutral pion was isolated in 1950, completing a trilogy of particles that were identified as the carrier particles predicted by Yukawa.

The availability of high energy particle accelerators in the middle fifties and early sixties, allowed the discovery of a multitude of strong interacting particles collectively known as hadrons, that formed groups of particles with similar properties [55]. The first formal group classification of the hadrons was given by Gell Man [16] and independently by Yuval Ne'Man [17] in the so-called "Eightfold Way", that classified hadrons into subgroups identified with octet representations of the SU(3) group. The classification of hadrons proposed by Gell Man was confirmed by the 1964 discovery of the omega-minus particle, predicted in 1962. After this

\footnotetext{
${ }^{1}$ a common name for protons and neutrons
} 
discovery Gell-Mann [20] and George Zweig [21,22] independently predicted that hadrons were composed of elementary fermions named as quarks [54].

The ever increasing energy of modern particle accelerators and the complete knowledge of the electromagnetic interaction between electrons and nucleons or nuclei were essential to prove the existence of the quarks as fundamental components of hadrons in general, and nucleons in particular. In 1968, the first results from deep inelastic scattering experiments demonstrated that protons and neutrons are composite structures made up of particles with fractional electric charge as well as neutral particles [56,57]. The charged particles were later identified with quarks predicted by Gell-Mann [20] and Zweig [21,22], and the neutral particles with the massless gluons, the carriers of the strong interaction between quarks in hadrons.

The above mentioned experimental and theoretical efforts resulted in the formalization and acceptance of the quantum field theory of strong interactions: quantum chromodynamics (QCD), within QCD the vast majority of physical hadrons are many-body, highly relativistic systems composed of light quarks and massless gluons [54, 85]. Quantum Chromodynamics (QCD) is a non-abelian gauge field theory (Yang and Mills theory [13] ) that describes the strong interactions of colored quarks and gluons fields. Quantum Chromodynamics is the $\mathrm{SU}(3)_{c}$ component of the $\mathrm{SU}(3)_{c} \mathrm{xSU}(2)_{L} \mathrm{xU}(1)_{Y}$ of the Standard Model of Particle Physics.

Quarks are strongly interacting fermions with spin $1 / 2$ and, by convention positive parity. The electric charges of the quarks are $-1 / 3$ and $2 / 3$. There are six different types of flavors of quarks: up (u), down (d), strange (s), charm (c), top (t) and bottom (b). In order to have antisymmetric wave functions (Pauli exclusion principle), the quarks carry color charges, which for convention are called: red (R), green $(\mathrm{G})$ and blue(B). The color of quarks is a quantum number that was first proposed by Fritzsch and Gell-Man [31] who identified the extra symmetry of QCD with the color symmetry. As a result of the color charges, quarks are said to be in the fundamental representation of the $\mathrm{SU}(3)_{c}$ color Lie group.

Gluons are massless gauge bosons, with spin 1 and two polarization states, which mediate color interactions among quarks. They, represented through non-Abelian gauge fields in QCD, are responsible for binding the quarks together. Gluons carry color charges and hence 
interact with each other even in the absence of quarks [40]. Gluons transform under the adjoint representation of the Lie group $\mathrm{SU}(3)_{c}[117]$.

Even though hadrons are composite systems of quarks and gluons, the understanding of the nuclear structure is mainly based on the nucleonic degrees of freedom. Hence, a nucleus of mass number $A$ and atomic number $Z$, is commonly represented as a system of A nucleons, $Z$ of which are protons and $(A-Z)$ neutrons. The main picture of nuclei as a composite system of nucleons is taken from the mean field approximation, on which each nucleon is modeled as an independent particle moving in the mean field potential of the remaining nucleons [80]. However, the picture breaks down when the momentum of the bound nucleon exceeds $k_{F} \simeq$ $250 \mathrm{MeV} / c$, where $k_{F}$ is the characteristic Fermi momentum of the nucleus described as a degenerate Fermi gas [28].

One of the major issues of modern nuclear physics is to understand the nuclear structure above the $k_{F}$ domain. The issue is directly related to understanding the nucleon-nucleon interaction at short distances inside the nucleus. The interaction prevents two nucleons from coming very close together demanding the existence of high momentum components in the nuclear ground state wave function. The close nucleon-nucleon interactions cannot be described in the context of nuclear mean field models and are commonly called multi-nucleon short-range correlations (SRC) [99].

Deep inelastic scattering (DIS) of leptons off the nucleus is one of the methods for probing nuclear structures at small distance scales. Before 1983, it was predicted that the quarks inside the nucleus followed the rules of the mean field model. According to which the quark-gluon dynamics in each nucleon was not influenced by the nuclear mean field. The fact that the quark momentum distributions for individual nucleons are modified in the nuclear medium was first observed by the European Muon Collaboration (EMC) experiment and is usually known as the EMC effect [38]. The discovery of the EMC effect is considered the starting event of a new era in nuclear QCD.

The importance of the high momentum properties of bound nucleon for nuclear EMC effects follows from the recent observations of apparent correlation between the medium modification of the quark momentum distributions and the strength of the two-nucleon short range 
correlations (SRCs) in nuclei $[97,100]$. In order to improve our knowledge of the role of the QCD interactions in the nuclear dynamics, it is crucial to understand the role of the SRCs in the EMC effects [75].

As a result of the QCD evolution of nuclear parton distributions functions(PDFs) ( see Eq. (1.3)), it is expected that at very large $Q^{2}$ the knowledge of the high momentum component of the bound nucleons becomes important because of the contribution of quarks with momentum fractions $(x)$ larger than the ones provided by an isolated nucleon (i.e. partons with $x>1)[125,126]$.

The same is true for the reliable interpretation of neutrino-nuclei scattering experiments in which case both the medium modification of PDFs as well as the realistic treatment of SRCs are essential. One of the main results of the experiments is the discrepancy found between measurements of $\sin ^{2} \theta_{w}\left(\theta_{w}\right.$ is the weak mixing angle) involving free particles and those involving bound nucleons in the nuclear medium, the so-called NuTev anomaly $[72,73,77,102]$. A possible explanation to the corresponding results is derived from the presence of more energetics protons in neutron rich, large A, asymmetric nuclei which implies that u-quarks are more modified than d-quarks, resulting in a negative correction for the experimental value of $\sin ^{2} \theta_{W}$ for bound nucleons [111].

All the above described areas of research require models to predict the high momenta and binding energies of bound nucleons in the nuclei. Such models are described through the nuclear spectral functions that define the joint probability of finding a nucleon in the nucleus with momentum p and removal (binding) energy $E_{m}$. With the advent of the Large Hadron Collider and expected construction of electron-ion colliders as well as several ongoing neutrino-nuclei experiments, the knowledge of such spectral functions will be an important part of the theoretical interpretation of the data involving nuclear targets.

The present dissertation is divided in four major chapters. In chapter 2, the theoretical framework for the multinucleon short-range correlation model for nuclear spectral functions is developed. The diagrammatic method of calculation of the spectral function is described, as well as the mathematical models for the spectral function in two approaches: Virtual nucleon and light front approximations. From the mathematical models of chapter 2, computational 
models to obtain numerical estimates for the spectral functions, density matrices, and nucleon momentum distributions are developed in chapter 3 . The numerical estimates of the spectral functions, density matrices, and nucleon momentum distributions are presented in chapter 4 . Finally, the main conclusions and an outline of future projects are included in chapter 5.

In the rest of chapter 1, the relationship between lepton-nucleus scattering cross section and the nuclear spectral function is presented. Then, a brief description of the EMC effect and its relation to $2 \mathrm{~N}$ SRC is included. The assumptions for modeling two and three nucleon shortrange correlations are also described. Finally, some definitions and the range of validity of the nuclear spectral functions, including the treatment of the relativistic effects and the model for 2N SRC center of mass motion are presented.

\subsection{Lepton-nucleus inclusive scattering cross section and nuclear spectral function}

In the lepton-nucleus inclusive scattering reaction $\left(l+A \longrightarrow l^{\prime}+X+(A-1)^{*}\right)$, shown in Fig.1.1(a), $\mathrm{X}$ is the hadron final state and (A-1) the recoil system of bound nucleons, and only the final lepton is detected.

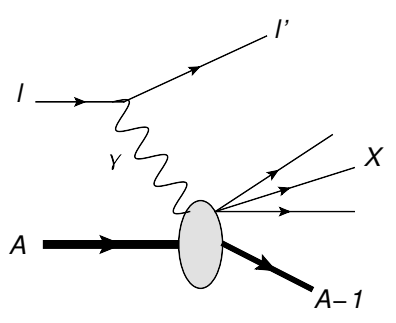

(a) Lepton-Nucleus Scattering

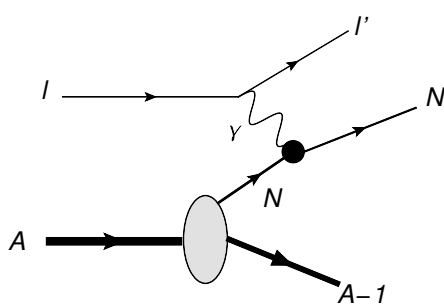

(b) Quasi-elastic Scattering

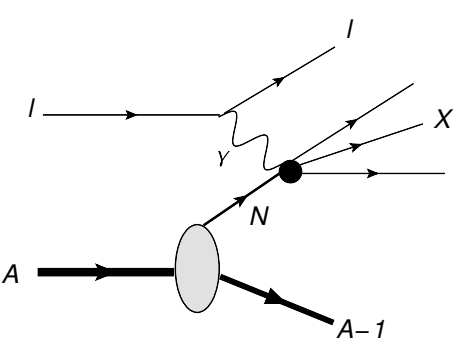

(c) Deep-Inelastic Scattering

Figure 1.1: Lepton-nucleus inclusive scattering.

In the one-boson exchange approximation, if 1 and l' are the same leptons ( electrons or muons) the reaction is mediated by either the weak neutral gauge boson, $Z^{0}$, or the photon $\gamma$. If $l$ and l' differs by one unit of charge, for instance (muon, neutrino) or (anti-muon, antineutrino), the reaction is mediated by the weak charged gauge bosons, $W^{-}$, or $W^{+}$.

The kinematic variables for the lepton-nucleus inclusive scattering in the lab frame (nucleus rest frame) are: $p_{A}=\left(M_{A}, 0\right)$, for the four-momentum vector of the nucleus of mass $M_{A}$; 
$k_{l}^{\mu}=\left(E_{l}, \mathbf{k}_{l}\right)$ and $k_{l^{\prime}}^{\mu}=\left(E_{l^{\prime}}, \mathbf{k}_{l^{\prime}}\right)$, for the four-momentum vectors of the initial 1 and final $l^{\prime}$ leptons, respectively. $q^{\mu} \equiv\left(q^{0}, \mathbf{q}\right)=\left(E_{l}-E_{l^{\prime}}, \mathbf{k}_{l}-\mathbf{k}_{l^{\prime}}\right)$, is the four-momentum vector of the gauge boson, with $Q^{2} \equiv-\left(q^{\mu}\right)^{2}$ defined as the virtuality of the gauge boson. If $q^{0} \ll M_{Z}, M_{W}$, where $M_{Z}\left(M_{W}\right)$ is the mass of the $\mathrm{Z}\left(W^{+}\right)$gauge boson, and l=l' (muon or electron) the reaction is defined by one-photon exchange.

Theoretical studies demonstrated that If $Q^{2} \geqslant M_{N}^{2}$, where $M_{N}$ is the mass of a nucleon. the size of the probe $\left(\sim 1 / Q^{2}\right)$ is smaller than the size of the nucleon, hence it can be considered that the lepton scatters off a bound nucleon inside the nucleus (see Fig.1.1(b) and (c)). In Fig 1.1 , it is assumed that the final state interactions of the hadron final state $(X)$ can be neglected. Such an assumption is referred to as the plane wave impulse approximation (PWIA) [39, 82]. The PWIA is justified for inclusive processes with large $Q^{2}\left(Q^{2} \geq 2 \mathrm{GeV}^{2}\right)$ such as deep inelastic scattering [113].

The total energy of the lepton-nucleon scattering (Fig. 1.1(b)) is defined as $W_{N}^{2} \equiv\left(p_{N}^{\mu}+\right.$ $\left.q^{\mu}\right)^{2}$. If $W_{N}^{2} \sim M_{N}^{2}$ then the hadron final state $\mathrm{X}$ is a nucleon and the corresponding leptonnucleus scattering is defined as quasi-elastic. If $W_{N}^{2}>\left(M_{N}+M_{\pi}\right)^{2}$, the hadron final state $\mathrm{X}$ may consist of a nucleon and a pion $(\pi)$ or baryonic excited resonances. In the region of deep inelastic scattering (DIS), $W_{N}>2.5 \mathrm{GeV}$ and $Q^{2}>2.0 \mathrm{GeV}^{2}$, the target nucleon breaks down in a final state $\mathrm{X}$ consisting of a collection of hadrons.

Deep inelastic lepton-nucleon scattering (DIS) has been fundamental in unveiling the structure of nucleons. In the parton model with very large $Q^{2}[26,29]$, the virtual photon interacts with one of the quarks of the bound nucleon (Fig 1.1(c)). Such a picture is relevant in the infinite momentum frame (IMF) of the nucleon. The lepton-nucleon center of mass frame is a good approximation to such IMF, on which the quarks (partons) within the nucleons are slowed down by Lorentz time dilation effects. In the IMF, therefore, the struck quark can be considered free, and characterized by the momentum fraction of the fast nucleon defined as the invariant Bjorken scaling variable $x=Q^{2} /(2 p q),(0 \leq x \leq 1)$, [26].

The one-photon exchange differential cross section for unpolarized lepton-nucleon inclusive inelastic scattering can be expressed through two independent nucleon structure func- 
tions $F_{1}^{N}$ and $F_{2}^{N}$ as $[11,23,24,101]$

$$
\left.\frac{d^{2} \sigma}{d E_{l^{\prime}} d \Omega_{l^{\prime}}}\right|_{l a b}=\left(\frac{d \sigma}{d \Omega}\right)_{M o t t} \frac{1}{v}\left[F_{2}^{N}\left(x, Q^{2}\right)+\frac{2 v}{M_{N}} \tan ^{2}\left(\theta_{l} / 2\right) F_{1}^{N}\left(x, Q^{2}\right)\right],
$$

where $v=q^{0}=Q^{2} /\left(2 M_{N} x\right)$, and $\left(\frac{d \sigma}{d \Omega}\right)_{M o t t}=\frac{\alpha^{2} \cos ^{2}\left(\theta_{l} / 2\right)}{4 E_{l}^{2} \sin ^{4}\left(\theta_{l} / 2\right)}$ is the Mott cross section for electronpoint charge scattering [4] , and $\theta_{l}$ the scattering angle. In the elastic scattering kinematics, $F_{1}^{N}$ and $F_{2}^{N}$ can be expressed as functions of the so called electric $\left(G_{E}\right)$ and magnetic $\left(G_{M}\right)$ form factors of the nucleon $[15,18,35,40]$.

In the inelastic scattering kinematics, $F_{1}^{N}$ and $F_{2}^{N}$ are functions of the two independent variables $x$ and $Q^{2}$. However within the partonic model of the nucleon, it was predicted that in deeps inelastic scattering (large $Q^{2}$ ):

$$
\begin{aligned}
& F_{1}^{N}\left(x, Q^{2}\right) \rightarrow F_{1}^{N}(x), \\
& F_{2}^{N}\left(x, Q^{2}\right) \rightarrow F_{2}^{N}(x),
\end{aligned}
$$

where the dimensionless nucleon structure functions, $F_{1,2}^{N}$ are independent of $Q^{2}$, that is independent of any mass scale (scale invariant), signaling the presence of free point-like quarks in the nucleons by satisfying the Bjorken scaling property [26].

The nucleon structure functions contain the information about the parton's (quark's) momentum distribution in the nucleon, namely

$$
\begin{aligned}
& F_{2}^{N}(x)=\sum_{i} e_{i}^{2} x f_{i}(x), \\
& F_{1}^{N}(x)=\frac{1}{2 x} F_{2}^{N}(x),
\end{aligned}
$$

where $e_{i}$ is the quark electric charge, and $f_{i}(x)$ is the parton longitudinal momentum distribution function in the infinite momentum frame, that is the probability that the struck quark $i$ carries a fraction $x$ of the nucleon momentum $p$. Hence, the nucleon structure function $F_{2}^{N}$ gives the weighted, by the square of the parton electric charge, probability of finding a parton in the nucleon that carries a fraction $x$ of the total nucleon momentum. 
The parton momentum distribution functions $f_{i}(x)$ are normalized as:

$$
\sum_{i^{\prime}} \int x f_{i^{\prime}}(x) d x=1
$$

where $i^{\prime}$ sums over all the partons, not just the charged ones that interact with the photon $[34,40,108]$.

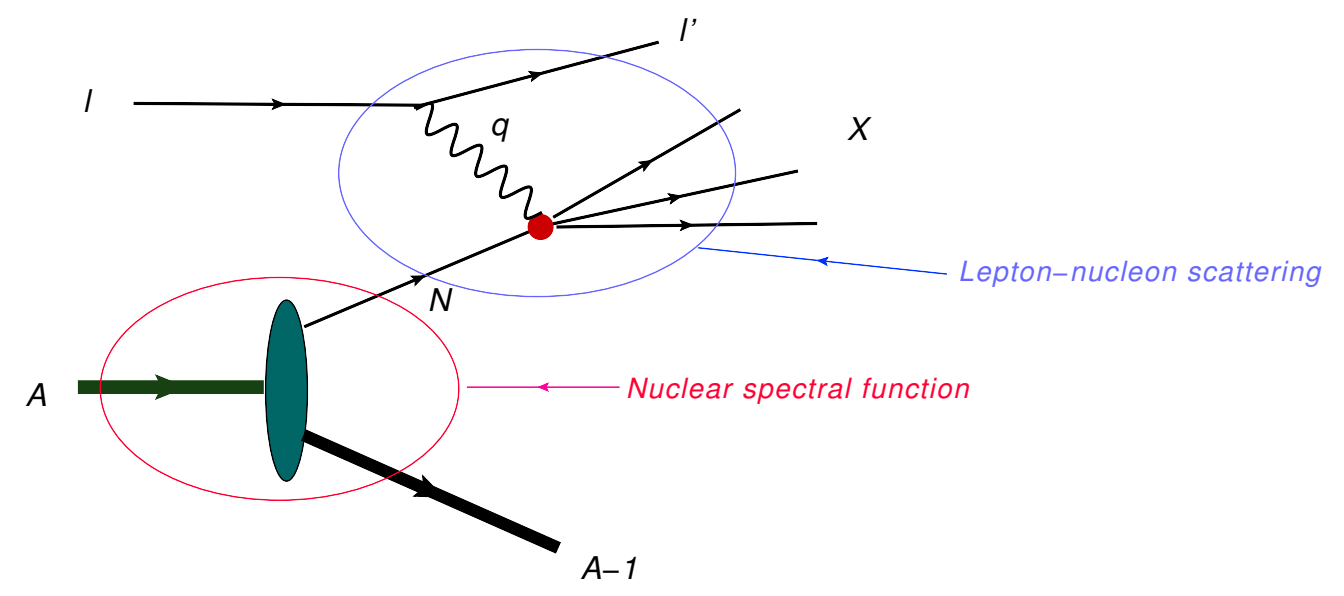

Figure 1.2: Lepton-nucleus deep inelastic inclusive scattering regions

Similar to the free nucleon case, the inclusive cross section for lepton-nucleus scattering can be expressed as follows

$$
\left.\frac{d^{2} \sigma}{d E_{l^{\prime}} d \Omega_{l^{\prime}}}\right|_{l a b}=\left(\frac{d \sigma}{d \Omega}\right)_{M o t t} \frac{1}{v}\left[F_{2}^{A}\left(x_{A}, Q^{2}\right)+\frac{2 v}{M_{A}} \tan ^{2}\left(\theta_{l} / 2\right) F_{1}^{A}\left(x_{A}, Q^{2}\right)\right]
$$

where $F_{1}^{A}$ and $F_{2}^{A}$ are the nuclear structure functions, that, within the above discussed plane wave impulse approximation (PWIA), can be expressed as a function of the nucleon structure functions, $F_{1}^{N}$ and $F_{2}^{N}$ and of the nuclear spectral function (as depicted in Fig. 1.2 for leptonnucleus deep inelastic inclusive scattering), by the following convolution integrals [70]

$$
F_{1}^{A}\left(x, Q^{2}\right)=\frac{A M_{N}}{M_{A}} \sum_{N} \int S_{A}^{N}\left(\mathrm{p}, E_{m}\right)\left[F_{1}^{N}\left(\tilde{x}, Q^{2}\right)+\frac{p_{\perp}^{2}}{2 M_{N} v} F_{2}^{N}\left(\tilde{x}, Q^{2}\right)\right] d^{4} p
$$




$$
\begin{aligned}
F_{2}^{A}\left(x, Q^{2}\right) & =\frac{A M_{N}}{M_{A}} \sum_{N} \int S_{A}^{N}\left(\mathrm{p}, E_{m}\right) \frac{v}{\tilde{v}}\left\{F _ { 2 } ^ { N } ( \tilde { x } , Q ^ { 2 } ) \left[\frac{(1+\cos \delta)^{2}}{M_{N}^{2}}\left(p^{+}+q^{+} \frac{p q}{q^{2}}\right)^{2}\right.\right. \\
& \left.\left.+\frac{\mathbf{p}_{\perp}^{2}}{2 M_{N}^{2}} \sin ^{2} \delta\right]\right\} d^{4} p,
\end{aligned}
$$

where $S_{A}^{N}\left(\mathrm{p}, E_{m}\right)$ is the nuclear spectral function which defines the joint probability of finding a nucleon in the nucleus with momentum $\mathrm{p}$ and removal energy $E_{m}, M_{A}$ is the mass of the nucleus, $x_{A}=Q^{2} /\left(2 q^{0} M_{A} / A\right), \tilde{v}=v+\left(p^{2}-M_{N}^{2}\right) /\left(2 M_{N}\right), \tilde{x}=Q^{2} /(2 p q)$, and $\cos \delta=v / \sqrt{v^{2}-q^{2}}$. The four vector momentum of the bound nucleon in the light-front coordinate frame is defined as $p^{\mu}=\left(p^{-}, p^{+}, \mathbf{p}_{\perp}\right)$, with $p^{+}=\mathrm{p}^{0}+\mathrm{p}_{z}, p^{-}=\mathrm{p}^{0}-\mathrm{p}_{z}$, and $\mathbf{p}_{\perp}=\left(\mathrm{p}_{x}, \mathrm{p}_{y}\right)$.

\subsection{The Nuclear European Muon Collaboration (EMC) Effect}

Since the binding energy of the nucleus is very small compared to the energy scales in deep inelastic scattering, it was assumed that, except for nucleon Fermi motion, the nucleus acted as a collection of slowly moving weakly bound nucleons, with their internal properties unchanged compared to the free nucleon case. Therefore the following ratio for the per nucleon inelastic structure functions $F_{2}^{A}$ to the inelastic structure functions $F_{2}^{p}$ and $F_{2}^{n}$ of a free proton and neutron

$$
R=A F_{2}^{A} /\left[Z F_{2}^{p}+(A-Z) F_{2}^{n}\right]
$$

was expected to rise for $\mathrm{x} \gtrsim 0.2$, and be about $1.2-1.3$ for $\mathrm{x}=0.65$, as it is shown in Fig. 1.3, in which Fermi motion corrections were included. Hence, it was expected that the nuclear deep inelastic inclusive scattering cross section will be completely defined by partonic distributions of free nucleons, with the nucleon Fermi motion as the only nuclear effect. It was also predicted that Eqs. (1.6) and (1.7), would give the same results for all nuclei. In summary, it was predicted that the nuclear cross section would be the sum of the cross sections of the number of nucleons inside the target $[38,76,108]$.

The above assumption was demonstrated to be wrong when the European Muon Collaboration (EMC) experiment discovered that in the muon-nucleus DIS, the ratio of the scattering 


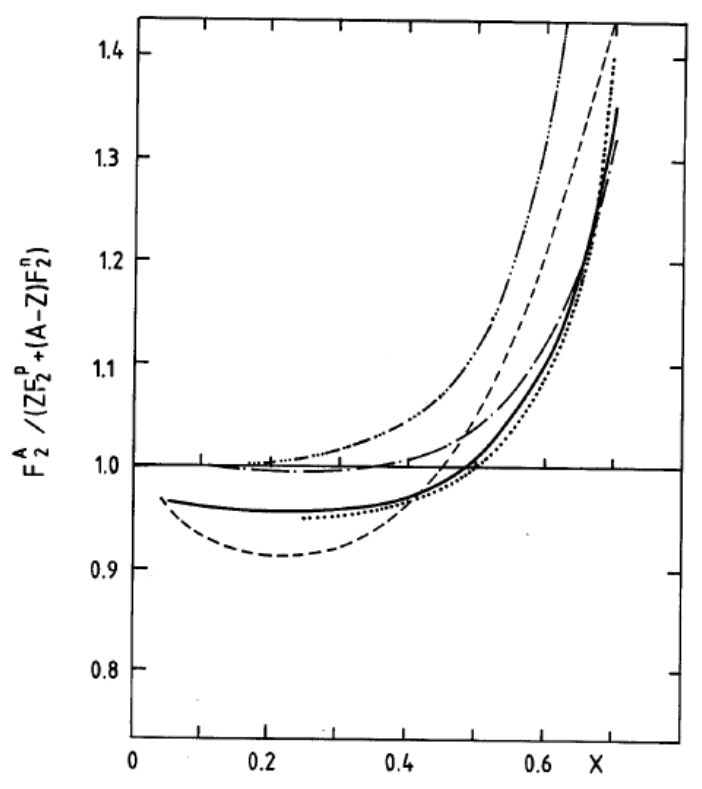

Figure 1.3: Theoretical predictions for the Fermi motion corrections of the bound nucleon structure functions $F_{2}^{N}$ for iron. Figure from [38].

cross section from nuclei to the deuteron, which is close to the ratio $\mathrm{R}$ in Eq. (1.8), was in strong disagreement with the predictions of Fig. 1.3. In the initial experiments, the ratio of the structure functions of iron nucleus and deuteron $\left(\mathrm{F}_{2}^{N}(\mathrm{Fe}) / \mathrm{F}_{2}^{N}(\mathrm{D})\right)$ was experimentally found to be decreasing and substantially different than unity in the region $0.05 \leq x \leq 0.65$ (see Fig. 1.4). The interpretation of the experimental results was that the inelastic structure functions of nucleons measured in nuclei are different from those of quasi-free nucleons in the deuteron [38, 42]. It was also found that this difference was growing with the mass number A of the nucleus, which indicated that the nucleon structure modification is proportional to the nuclear density. The modification of the quark momentum distribution of bound nucleons in the nucleus, as compared to that of free nucleons, became known as the nuclear European Muon Collaboration ( EMC) effect. 


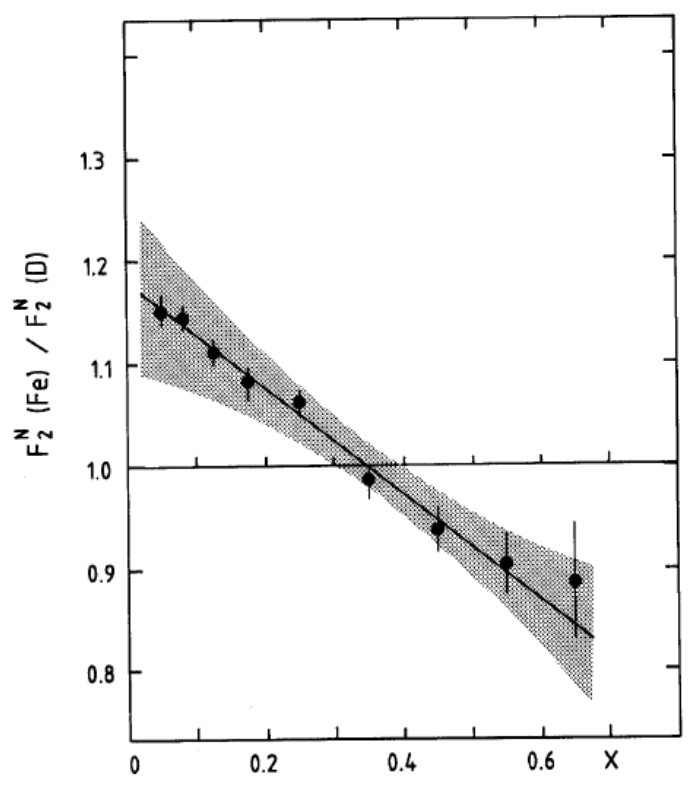

Figure 1.4: EMC experimental results for the ratio of the structure functions of iron nucleus and deuterium $\left(\mathrm{F}_{2}^{N}(\mathrm{Fe}) / \mathrm{F}_{2}^{N}(\mathrm{D})\right)$. Figure from [38].

Recent measurements at the Jefferson lab (shown in Fig. 1.5 for Carbon) verified the nuclear EMC effect with unprecedented accuracy for a wide range of nuclei, confirming that the EMC ratio $\mathrm{R}_{E M C}=2\left(\sigma_{e A}\right)_{i s} /\left(\mathrm{A} \sigma_{e D}\right)$ ( where $\left(\sigma_{e A}\right)_{i s}$ is the average cross section for isoscalar nucleon and $\sigma_{e D}$ is the cross section for deuteron) is below unit for all the targets studied [91].

\subsection{Multinucleon short-range correlations in nuclei}

The main theoretical picture that describes the bulk properties of nuclei, is that the nucleons are independent particles moving in an average or mean field generated by the remaining $(A-$ 1) nucleons in the nucleus. As a result each nucleon is independent of the exact instantaneous position of all other nucleons. The simple mean field model has been successful in correctly 


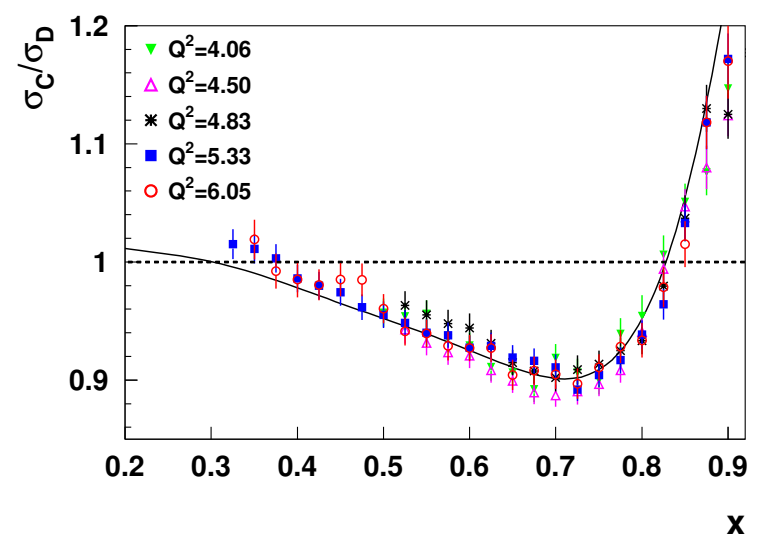

Figure 1.5: Carbon EMC ratios for the highest $Q^{2}$ settings $\left(Q^{2}\right.$ quoted at $\left.\mathrm{x}=0.75\right)$. Uncertainties are the combined statistical and point-to-point systematic. The solid curve is the SLAC fit to the Carbon EMC ratio. Figure from [91].

predicting all nuclear magic numbers, as well as in describing a large amount of nuclear data $[14,19,50]$.

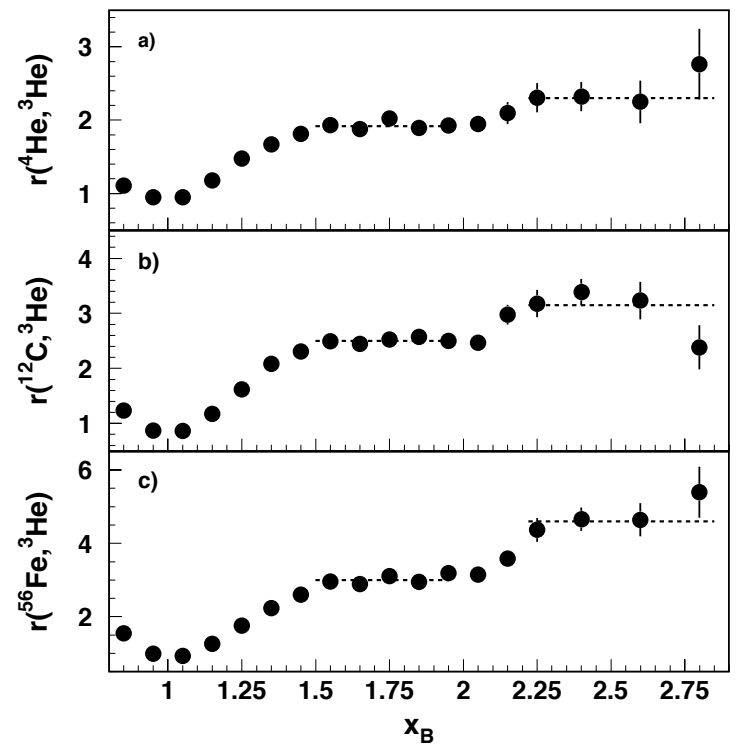

Figure 1.6: $2 \mathrm{~N}$ and $3 \mathrm{~N}$ scaling regions for nucleus A. Figure from Ref. [84]

The nuclear shell model, based on the above described mean field picture of the nucleus, is valid for long range ( $\geq 2 \mathrm{fm}$ ) mutual separation of nucleons, which in the momentum space corresponds to the nucleon momentum being less that $k_{F} \sim 250 \mathrm{MeV} / c$ ( [60]. The nuclear shell model however was found to break down for inter nucleon distances smaller than $2 r_{N}$, 
where $r_{N} \approx 0.85 \mathrm{fm}$ is the radius of the nucleon, on which two nucleons start to overlap and the notion of the mean field become invalid. Their dynamics are mainly defined by the NN interaction at short distances which is dominated by the tensor interaction $(\sim 0.8-1.2 \mathrm{fm})$ and repulsive core $(\leq 0.5-0.7 \mathrm{fm})[32,60,86]$. Such configurations are generally referred to as $2 \mathrm{~N}$ Short Range Correlations (SRCs) in the nucleus [46, 48, 89, 99, 133].

Theoretical analysis show that the nucleons belonging to such 2N SRCs have large (greater than $k_{F}$ ) relative momentum and low (smaller than $k_{F}$ ) center of mass momentum. There are also lower probability configurations such as three (3N) or multi-nucleon (MN) SRCs, a very important high density structures present in the ground state wave function of the nucleus, responsible for high momentum nucleons much above the Fermi momentum $k_{F}$. Any experiment designed to access such MN SRCs must probe the bound nucleon in the nucleus at very large momenta. Lepton-nucleus scattering, at large values of the Bjorken parameter, $x_{B}$, is the most appropriate experiment to prove such MN SRC nuclear structures.

The kinematic region for lepton-free nucleon scattering is $0<x_{B}<1$, whereas for bound nucleon in a nucleus $\mathrm{A}$ is $0<x_{B}<\mathrm{A}$. It is expected that scattering from $\mathrm{j}$-nucleon SRC will dominate at $\mathrm{j}-1<x_{B}<\mathrm{j}[49]$. If a lepton scatters off the nucleon from $\mathrm{j}$-nucleon SRCs, then it is expected that the cross section ratio

$$
R\left(A_{1}, A_{2}\right)=\frac{\sigma\left(A_{1}, Q^{2}, x_{B}\right) / A_{1}}{\sigma\left(A_{2}, Q^{2}, x_{B}\right) / A_{2}}
$$

where $\sigma\left(A_{1}, Q^{2}, x_{B}\right)$ and $\sigma\left(A_{2}, Q^{2}, x_{B}\right)$ are the inclusive lepton scattering cross sections of nucleus $A_{1}$ and $A_{2}$ respectively, will scale, that is to be constant. The scaling results from the dominance of MN SRCs in the high momentum component of the nuclear wave function. Hence, plateaus are expected in the ratio of the inclusive cross sections of heavy nucleus to light nuclei such as ${ }^{3} \mathrm{He}$, showing that the momentum distributions at high momenta have the same shape for all nuclei differing only by a scale factor $[43,49,78]$.

Recent experimental studies of high energy $e A$ and $p A$ processes $[64,74,78,84,85,90$, $103,119]$ resulted in a significant progress in understanding the dynamics of 2N SRCs in nuclei. The series of electron-nucleus inclusive scattering experiments $[78,84,103]$ have confirmed the 
prediction of the scaling for the ratios of inclusive cross sections of a nucleus to the deuteron $\left({ }^{3} \mathrm{He}\right.$ ) in the kinematic region $x_{B}>1$ (dominated by the scattering from the bound nucleons with momenta $p>k_{F} \sim 250 \mathrm{MeV} / c$ ) Within the 2N SRC model, these ratios allowed to extract the parameter $a_{2}(A, Z)$ which characterizes the probability of finding $2 \mathrm{~N}$ SRC in the nucleus relative to the deuteron. Results of the above mentioned experiments with $Q^{2} \geq 1.5 \mathrm{GeV}^{2}$, are shown in Fig. 1.6.The cross section ratios in Fig. 1.6 scales initially in the region $1.5<\mathrm{x}<2.0$, which indicates dominance of $2 \mathrm{~N}$ SRC in this region, and scales a second time for $\mathrm{x}>2.25$, indicating dominance of 3N SRC.

\subsubsection{Strong Correlation Between Nucleon-Nucleon Short Range Correlations and the EMC Effect}

Since both the EMC effect and the 2N SRCs depend on the mass number A and on the nuclear density, it was predicted that they were strongly correlated [92]. The prediction was probed by experiments on which the correlation between the strength of the nuclear EMC effect and the strength of 2N SRCs was observed as it is shown in Fig. 1.7 [97]. The strength of the EMC effect

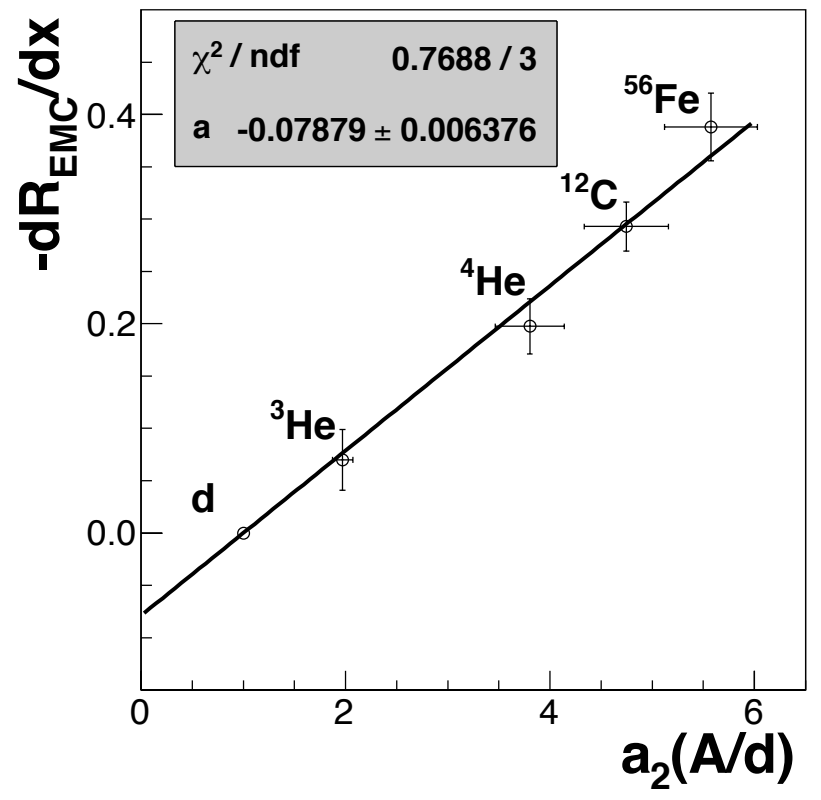

Figure 1.7: The EMC slopes versus the SRC scale factors. Figure from Ref. [97] 
for a nucleus A in Fig. 1.7 is represented by the slope of the EMC ratio $\left(\mathrm{R}_{\mathrm{EMC}}\right)$ of the per-nucleon deep inelastic cross section of nucleus A relative to the deuteron, $\mathrm{dR}_{\mathrm{EMC}} / \mathrm{dx}$, in the region 0.35 $\leq \mathrm{x} \leq 0.7$ [92]; and the strength of the 2N SRCs is represented by the nuclear scale factor of the nucleus A relative to the deuteron $\mathrm{a}_{2}(\mathrm{~A} / \mathrm{d})$, which represents the probability of having $2 \mathrm{~N}$ SRCs in the nucleus A.

The EMC-SRC correlation is a very important experimental result that provides new insight into the origin of the EMC effect. Since the SRC structure in the nucleus implies high momentum bound nucleons, it indicates that the EMC effect is only the result of the high momentum component of the nuclear wave function, so that the possible modification of the parton distributions in nucleons in the nucleus occurs only in nucleons belonging to SRCs [107, 108].

Hence, a further understanding of the dynamical origin of the observed EMC-SRC correlation requires a theoretical model for the nuclear spectral function, $S_{A}^{N}\left(\mathrm{p}, E_{m}\right)$, that describes the $2 \mathrm{~N}$ and $3 \mathrm{~N}$ SRCs in a consistent way. The model will allow the calculation of the cross sections for lepton -nucleus scattering from Eqs. (1.5), (1.6), and (1.7). Thus, the main motivation of the research presented in this dissertation was to develop a self consistent theoretical model for calculation of the nuclear spectral functions in the domain of $2 \mathrm{~N}$ and $3 \mathrm{~N}$ short range correlations. One of the important requirements in developing such models was that the calculated spectral functions should include all recent findings that have been made in experimental and theoretical studies of SRCs in nuclei as those described in the following sections (1.3.2 and 1.3.3) of the present chapter.

\subsubsection{Model for $2 \mathrm{~N}$ and $3 \mathrm{~N}$ short-range correlations in nuclei}

Despite impressive recent progress in ab initio calculations of nuclear structure for the mean field (shell) model (see e.g. Ref. [118]), their relevance to the development of the spectral functions at large momenta and removal energies, where the dominance of SRCs is expected, is rather limited. Not only the absence of relativistic effects but also the impossibility of identifying the relevant nucleon-nucleon $(\mathrm{NN})$ interaction potentials makes such a program unrealistic. One way for progress is to develop theoretical models that use the short-range NN correlation approach in the description of the high momentum part of the nuclear wave func- 


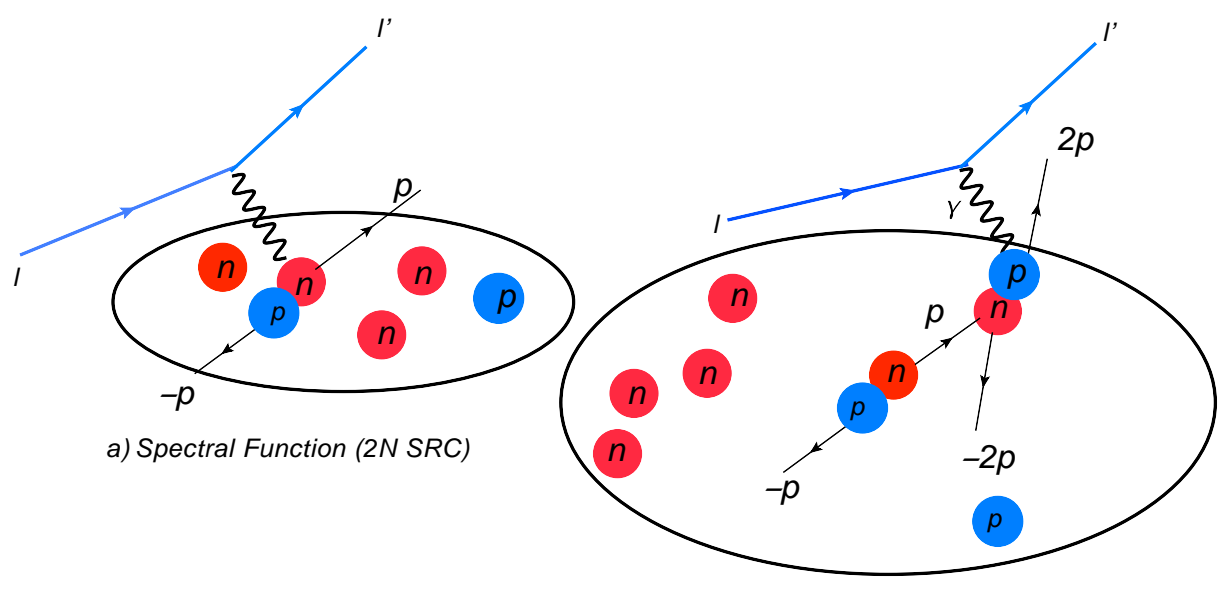

b) Spectral Function (3N SRC)

Figure 1.8: 2N and 3N SRC models for nuclear spectral functions

tion (see, e.g., $[36,45,52,105,109,110,116,124,128,129])$. In such an approach, it is possible to consider the empirical knowledge of SRCs acquired from different high energy scattering experiments thus reducing in some degree the theoretical uncertainty related to the description of high momentum nucleon in the nucleus.

The main goal of chapter 2 of the present dissertation is to develop a model for high momentum nuclear spectral function using the several phenomenological observations obtained in recent years in studies of the properties of two-nucleon SRCs $[78,84,85,88,90,103,106,119$, 130,131]. A model is first developed describing the nuclear spectral function at large momenta and missing energies dominated by $2 \mathrm{~N}$ SRCs with their center of mass motion generated by the mean field of the $A-2$ residual nuclear system.

Even though some experiments have shown evidence for 3N SRCs [84], there are other experiments [103] that did not see such evidence. Considering the experimental ambiguity, a theoretical framework for calculating the contribution of 3N SRCs to the nuclear spectral function is developed, using a model in which such correlations are generated by two sequential $2 \mathrm{~N}$ short range correlations as it is shown in Fig. 1.8. Hence, the phenomenological knowledge of the properties of $2 \mathrm{~N}$ SRCs is sufficient to calculate both the $2 \mathrm{~N}$ and $3 \mathrm{~N}$ SRC contributions to the model of nuclear spectral function. 
The model is assumed to be valid for nucleon momenta $k_{s r c} \leq p \leq 1000 \mathrm{MeV} / \mathrm{c}$, where $k_{s r c}$ is a momentum characteristic to the $2 \mathrm{~N}$ SRC. It is sufficiently large that $2 \mathrm{~N}$ SRCs can be factorized from residual mean field interaction. As a result the model will have limited validity in the transitional region of $k_{F} \leq p<k_{s r c}$ where the role of the long-range correlations are more relevant.

\subsubsection{Phenomenology of two nucleon short-range correlations in nuclei}

High energy semi-inclusive experiments $[85,90]$ probed for the first time the isospin composition of 2N SRCs, observing strong (by factor of 20) dominance of the $p n$ SRCs in nuclei, as compared to the $p p$ and $n n$ correlations, for internal momentum range of $\sim 250-650 \mathrm{MeV} / c$. The experimental results are understood by considering the dominance of the tensor forces in the NN interaction at the momentum range corresponding to the average nucleon separations of $\sim 1.1 \mathrm{fm}[82,85]$. The tensor interaction projects the NN SRC part of the wave function to the isosinglet - relative angular momentum, $L=2$, state, almost identical to the high momentum part of the $D$-wave component of the deuteron wave function. As a result $p p$ and $n n$ components of the NN SRC are strongly suppressed since they are dominated by the central NN potential with relative angular momentum $L=0[33,60,86]$.

On the basis of the above observation of the strong dominance of $p n$ SRCs, it was predicted that single proton or neutron momentum distributions in the $2 \mathrm{~N}$ SRC domain are inversely proportional to their relative fractions in nuclei $[104,112]$. The prediction is in agreement with the results of variational Monte-Carlo calculation of momentum distributions of light nuclei [120] as well as for medium to heavy nuclei following the SRC model calculations of Ref. [124]. The recent finding of the $p n$ dominance in heavy nuclei (up to ${ }^{208} \mathrm{~Pb}$ ) [119] validates the universality of the above prediction for the whole spectrum of atomic nuclei. The inverse proportionality of the high momentum component to the relative fraction of the proton or neutron is important for asymmetric nuclei and they need to be included in the modeling of nuclear spectral functions in the 2N SRC region.

The $p n$ dominance in the SRC region and its relation to the high momentum part of the deuteron wave function makes the studies of the deuteron structure at large internal momenta 
a very important part for the SRC studies in nuclei. In this respect, the recent experiments $[96,127]$ and planned new measurements [115] of high energy exclusive electro-disintegration of the deuteron opens up new possibilities in the extraction of the deuteron momentum distribution at very large momenta. The measured distributions can then be utilized in the calculation of the nuclear spectral functions in the multi-nucleon SRC region.

Finally, another progress relevant to the SRC studies was the extraction of the center of mass momentum distribution of $2 \mathrm{~N}$ SRCs from the data on triple coincidence scattering in $A(p, p p n) X[79]$ and $A\left(e, e^{\prime}, p n\right) X[87,121]$ reactions. The Gaussian form and the width of the extracted distributions were in a good agreement with the predictions made in Ref. [52], which were based on the estimate of the mean kinetic energy of the NN pair in shell-model description of nuclei. Similar results have been also obtained within the correlated wave function method of Ref. [122].

\subsection{Nuclear spectral function model}

The above discussed phenomenology will provide the necessary empirical input for modeling nuclear spectral functions in the SRC region. The model for high momentum nuclear spectral function, developed in chapter 2 of this dissertation, have two regions determined by the range of momentum considered. For momenta below the Fermi momentum, $k_{F}$, a mean field spectral function is constructed by using a nonrelativistic approach to estimate the ground state wave functions [80]. For momenta above $400 \mathrm{MeV}$, a relativistic multi-nucleon short range correlation model of the spectral function is obtained, which describes the high momentum and high missing energy of two and three nucleons in short range correlations (2N and 3N SRC), for symmetric and asymmetric nuclei.

Since the domain of multi-nucleon SRCs is characterized by the relativistic momenta of the probed nucleon, special care should be given to the treatment of relativistic effects. To identify the relativistic effects, in Sec.2, the nuclear spectral function is defined as a quantity which is extracted in the semi-exclusive high energy process whose scattering amplitude can be described through the covariant effective Feynman diagrams. The covariance here is important to consistently trace the relativistic effects related to the propagation of the bound nucleon. 
Then, the part of the covariant diagram which reproduces the nuclear spectral function is precisely identified. Two approaches are adopted for modeling the nuclear spectral function: virtual nucleon and light-front approximations, general features of which are described in Sec.2.1. Section 2.2 outlines the calculation of nuclear spectral functions using the effective Feynman diagrammatic method, identifying the diagrams corresponding to the mean field, 2N SRC with center of mass motion and 3N SRC contributions.In Secs.2.3 and 2.4, the detailed derivation of the nuclear spectral functions within the virtual nucleon and the light-front approximations are presented.

Chapters 3 and 4 are dedicated to the development of computational models to evaluate nuclear spectral functions, density matrices and momentum distributions for a wide range of light and heavy nuclei. The results of the spectral function models will be compared with $a b$ initio, nonrelativistic quantum Monte Carlo calculations (QMC) (for A $\leq 11$ ) $[51,67,71,120]$. The values of $a_{2}$ and $a_{3}$ are also predicted, which represent the probability of having $2 \mathrm{~N}$ and $3 \mathrm{~N}$ SRCs in the nuclear ground state wave function, respectively. 


\section{CHAPTER 2}

\section{Multinucleon short-range correlation model for nuclear spectral functions: Theo- retical framework}

The definition of nuclear spectral functions used in the present dissertation is derived by identifying a nuclear "observable" which can be extracted from the cross section of the large momentum ( $\gg$ nucleon mass) transfer semi-inclusive $h+A \rightarrow h^{\prime}+N+(A-1)^{*}$ reaction in which the $N$ can be unambiguously identified as a struck nucleon carrying almost all the energy and momentum transferred to the nucleus by the probe $h$. The reaction is specifically chosen to be semi-inclusive so that it allows, in the approximation in which no final state interactions are considered, to relate the missing momentum and energy of the reaction to the properties of bound nucleon in the nucleus. When those conditions are satisfied the extracted "observable", referred to as a nuclear spectral function, represents a joint probability of finding a bound nucleon in the nucleus with given missing momentum $p$ and removal energy $E_{m}$.

The models of nuclear spectral functions developed in the present dissertation must be relativistic, since they will be used to describe bound nucleons with high momenta and high removal energies. The relativistic effects are accounted for by using effective Feynman diagrammatic approaches similar to those developed in Refs. [53, 61, 68, 81]. One problem associated with the relativistic domain is the existence of vacuum fluctuations that implies the existence of negative energy components which are not related to the probability amplitude of finding a nucleon with a given momentum in the nucleus, and therefore, are not components of the nuclear spectral function.

Chapter 2 is organized as follows. The two approaches to deal with vacuum fluctuations: virtual nucleon (VN) and light-front (LF) approximations are described in section 2.1. Section 2.2 outlines the modeling of nuclear spectral functions using the effective Feynman diagrammatic method, identifying the diagrams and the corresponding amplitudes which represent partial contributions to the total amplitude by nucleons in the nuclear mean field, in two nucleons short-range correlation with center of mass motion, and in three nucleons short-range correlation. The steps for the calculation of the models of the nuclear spectral function are 
also defined in section 2.2. Sections 2.3 and 2.4 include the detailed derivations of the models of nuclear spectral functions within the virtual nucleon and the light-front approximations respectively. Section 2.5 summarizes the results of the chapter.

\subsection{Approaches to deal with vacuum fluctuations}

The vacuum fluctuations are a purely relativistic phenomena associated with the existence of particles and antiparticles that can pop out from the vacuum and then disappear into it. The notion of the antiparticle was first proposed by Dirac in 1932 to explain the solutions of the Klein-Gordon equation with negative energies. The positron was predicted as the antiparticle of the electron with a positive charge and a negative energy.

Stuckelberg in 1941 and Feynman in 1948 proposed that a negative energy solution describes a particle which propagates backward in time, or equivalently a positive energy antiparticle propagating forward in time. This concept was incorporated in the Feynman diagrams, a very powerful method of calculation in quantum field theory. With the help of Feynman diagrams it is possible to show that, for certain time ordering of process, a pair particle- antiparticle may appear spontaneously from the vacuum, and unless there is some external energy carried by a probe, the pair will disappear back into it [40].

The models, developed in the present dissertation, for relativistic nuclear spectral functions are derived from an effective Feynman diagrammatic approach for calculation of the $h+A \rightarrow h^{\prime}+N+(A-1)^{*}$ reactions (Fig.2.1) derived in Refs. [53, 61, 68]. In the approach the covariant Feynman scattering amplitude is expressed through the effective nuclear vertices, vertices which are related to the scattering of the probe $h$ with the bound nucleon, as well as vertices related to the final state of the reaction.

The nuclear vertices related with the bound nucleon can not be associated $a$ priori with the single nucleon wave function of the nucleus, since they contain negative energy components which are related to the vacuum fluctuations rather than the probability amplitude of finding nucleon with given momentum in the nucleus. The problem of vacuum fluctuations is illustrated in the diagrammatic representation of the reaction shown in Fig. 2.1, in which the covariant diagram (a) is a sum of two non-covariant time ordered scattering diagrams (b) and 


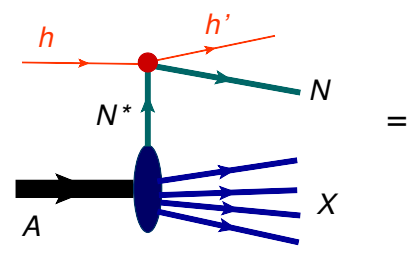

(a)

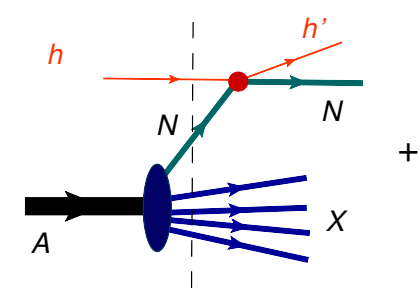

(b)

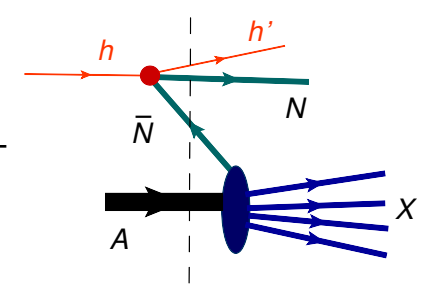

(c)

Figure 2.1: Representation of the covariant Feynman amplitude through the sum of the time ordered amplitudes. Panel (b) corresponds to the scenario in which first, the bound nucleon is resolved in the nucleus which interacts with the incoming probe $h$. In panel (c), initially, the incoming probe produces a $\bar{N} N$, anti-nucleon and nucleon pair, with subsequent absorption of the $\bar{N}$ anti-nucleon in the nucleus.

(c). Here, for the calculation of the Lorentz invariant amplitude of Fig. 2.1(a), the Feynman diagrammatic rules (given in Ref. [68]) can be applied. However the nuclear spectral function can only be formulated for the diagram of Fig. 2.1(b), where the time ordering is such that it first exposes the nucleus as being composed of a bound nucleon and residual nucleus, followed by an interaction of the incoming probe $h$ off the bound nucleon. The other time ordering [Fig. 2.1(c)] presents a very different scenario of the scattering in which the probe produces a $\bar{N} N$, anti-nucleon and nucleon pair with subsequent absorption of the $\bar{N}$ anti-nucleon in the nucleus. The later is usually referred to as a $Z$-graph and is not related to the nuclear spectral function. It is worth noting that the $Z$-graph contribution is a purely relativistic effect and does not appear in the non-relativistic formulation of the nuclear spectral function. The $Z$-graph contribution however increases with an increase of the momentum of the bound nucleon [see e.g. Ref. [36]].

The above discussion indicates that while defining the nuclear spectral function is straightforward in the non-relativistic domain (no $Z$-graph contribution), its definition becomes increasingly ambiguous with an increase of the momentum of the bound nucleon. The ambiguity is reflected in the lack of uniqueness in defining the nuclear spectral function in the domain where it is expected to probe SRCs inside the nucleus.

In the present dissertation two approaches are considered to deal with the vacuum fluctuations, so that a unique definition of the nuclear spectral function from the covariant scattering amplitude is obtained. In the first approach, referred to as the virtual nucleon (VN) 
approximation, the $Z$-graph contribution is neglected $[93,98]$. In the second approach, referred to as the light front (LF) approximation, the $Z$-graph contribution is kinematically suppressed $[25,36,46,66]$.

\subsubsection{Virtual nucleon (VN) approximation approach}

In the virtual nucleon $(\mathrm{VN})$ approximation approach, the $Z$-graph contribution is neglected by considering only the positive energy pole for the bound nucleon propagator in the nucleus. The energy and momentum conservation in the $\mathrm{VN}$ approach requires the interacting nucleon to be virtual which renders certain ambiguity in treating the propagator of the bound nucleon. The ambiguity is solved by recovering the energy and momentum of the interacting nucleon from kinematic parameters of on-shell spectators [see Ref. [37] for general discussion of the spectator model of relativistic bound states].

The advantage of the $\mathrm{VN}$ approximation is that the spectral function is expressed through the nuclear wave function defined in the rest frame of the nucleus which in principle can be calculated using conventional NN potentials.

One shortcoming of the $\mathrm{VN}$ approximation is that while it satisfies the baryonic number conservation law, the momentum sum rule is not satisfied reflecting the virtual nature of the probed nucleon in the nucleus.

\subsubsection{Light-front (LF) approximation approach}

The light-front representation of the space-time was first proposed by Dirac [10] as a form of relativistic Hamiltonian dynamics. The three forms of Hamiltonian dynamics described by Dirac were: the instant form, the front form, and the point form. These forms differ in the hypersphere on which the fields are analyzed [62]. In the front form the hypersphere is a plane tan-

gent to the light-cone, that is the three-dimensional surface in space-time formed by a plane wave frame advancing with the velocity of light, such a surface was called a front by Dirac. 
The space-time coordinates in the light-front are defined as [62,63]:

Lorentz Vectors: The contravariant four-vectors of position $x^{\mu}$ are written as

$$
x^{\mu}=\left(x^{+}, x^{-}, x^{1}, x^{2}\right)=\left(x^{+}, x^{-}, \mathbf{x}_{\perp}\right)
$$

Its time-like and space-like components are related to the instant form by:

$$
\begin{aligned}
& x^{+}=x^{0}+x^{3}, \\
& x^{-}=x^{0}-x^{3},
\end{aligned}
$$

respectively, and referred to as the light-front time and light-front longitudinal position. The null plane is defined by $\mathrm{x}^{+}=0$, that is, this condition defines the hyperplane that is tangent to the light-cone. The initial boundary conditions for the dynamics in the light-front are defined on this hyperplane. The axis $\mathrm{x}^{+}$is perpendicular to the plane $\mathrm{x}^{+}=0$. Therefore a displacement of such hyperplane for $x^{+}>0$ is analogous to the displacement of a plane in $t=0$ to $t>0$ of the four-dimensional space-time. With this analogy, $\mathrm{x}^{+}$is recognized as the time in the lightfront, or equivalently, as the light-front time $\tau=x^{0}+x^{3}$. The contravariant four-vectors of momentum $p^{\mu}$ are written as

$$
p^{\mu}=\left(p^{+}, p^{-}, p^{1}, p^{2}\right)=\left(p^{+}, p^{-}, \mathbf{p}_{\perp}\right)
$$

Its time-like and space-like components are related to the instant form by:

$$
\begin{aligned}
& p^{+}=p^{0}+p^{3}, \\
& p^{-}=p^{0}-p^{3} .
\end{aligned}
$$

The scalar product between two 4-vectors is defined by:

$$
x p=x^{\mu} p_{\mu}=x^{+} p_{+}+x^{-} p_{-}+x^{1} p_{1}+x^{2} p_{2}=\frac{1}{2}\left(x^{+} p^{-}+x^{-} p^{+}\right)-\mathbf{x}_{\perp} \mathbf{p}_{\perp} .
$$


if $x=p$, then the following very useful relation is obtained:

$$
p^{+}=\frac{M^{2}+\mathrm{p}_{\perp}^{2}}{p^{-}},
$$

where $M$ is the mass of the particle with momentum $p$.

The four-dimensional phase space differential element is defined as

$$
d^{4} x=d x^{0} d^{2} \mathbf{x}_{\perp} d x^{3}=\frac{1}{2} d x^{+} d x^{-} d^{2} \mathbf{x}_{\perp}
$$

In the light-front (LF) approximation the nuclear spectral function is defined on the light front which corresponds to a reference frame in which the nucleus has infinite momentum. Weinberg [25] showed that in the infinite momentum frame all diagrams with negative energy, like the the $Z$-graph, are kinematically suppressed. Then, as a result, the invariant sum of the two light-cone time ordered amplitudes in Fig. 2.1 is equal to only the contribution from the graph of Fig. 2.1 (b). Therefore, the boost invariant LF nuclear spectral function defines the joint probability of finding a nucleon in the nucleus with given light-front momentum fraction, transverse momentum, and invariant mass [58].

It is worth noting that the LF approximation satisfies the baryonic conservation law, as well as the momentum sum rules, thus providing a better framework for studies of the effects associated with the nuclear medium modification of interacting particles.

The LF approach developed in the present dissertation is field-theoretical, that is the Feynman diagrams are constructed with effective interaction vertices and the spectral functions are extracted from the imaginary part of the covariant forward scattering nuclear amplitude. Another approach, in LF approximation, is the construction of the nuclear spectral function based on the relativistic Hamiltonian dynamics representing the interaction of fixed number on-mass shell constituents [132]. 


\subsection{Diagrammatic approach for modeling nuclear spectral functions}

The application of the Feynman diagrammatic rules of Ref. [68] to obtain the mathematical model of nuclear spectral functions starts by identifying the effective interaction vertices $\hat{V}$ shown in Fig. 2.2, such that the imaginary part of the covariant forward scattering nuclear amplitude will reduce to the nuclear spectral function either in VN or LF approximations. The specific form of the vertices can be established by considering the amplitude of Fig. 2.1(b), taking into account the kinematics of the mean field, the $2 \mathrm{~N}$, and the $3 \mathrm{~N}$ SRC scattering within VN and LF approximations, and with subsequent factorization of the scattering factors related to the external probe $h$. As a result the $\hat{V}$ vertices will be different for mean field, $2 \mathrm{~N}$, and $3 \mathrm{~N}$ SRCs. They will also depend on the VN or the LF approximations used to calculate the scattering amplitude.

In applying the diagrammatic approach, the forward nuclear scattering amplitude $A$ can be expressed as a sum of the mean field and the multinucleon SRC contributions as presented in Fig. 2.2, with (a), (b), and (c) corresponding to the contributions from nucleons in the nuclear mean-field, and from the $2 \mathrm{~N}$ and the $3 \mathrm{~N}$ short-range correlations respectively:

$$
A=A^{M F}+A^{2 N}+A^{3 N},
$$

where $A^{M F}, A^{2 N}$, and $A^{3 N}$ correspond to the contributions from the diagrams of Fig. 2.2 (a)2.2(c) respectively.

Since the mean field contribution is dominated by the momenta of interacting nucleon below the characteristic Fermi momentum, $k_{F}$, it is valid to approximate the corresponding nuclear spectral function to the result following from nonrelativistic calculation. Hence, both the $\mathrm{VN}$ and the LF approximations are expected to give very close results.

For the $2 \mathrm{~N}$ and $3 \mathrm{~N}$ SRCs, the momenta of probed nucleon is in the range $k_{F}<p \leq 600-$ $1000 \mathrm{MeV} / c$ and the nonrelativistic approximation is increasingly invalid. 


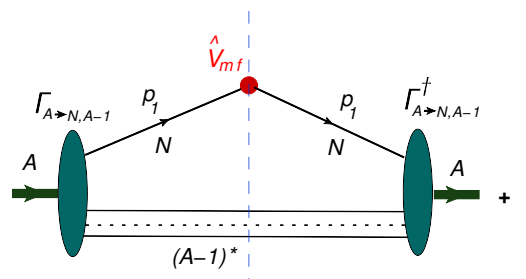

(a)

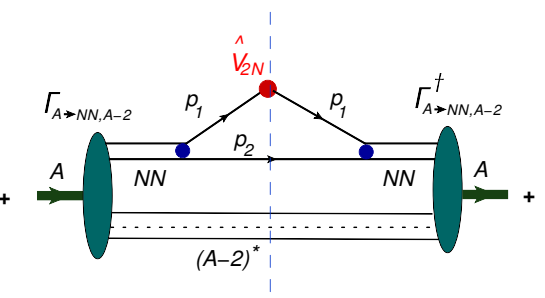

(b)

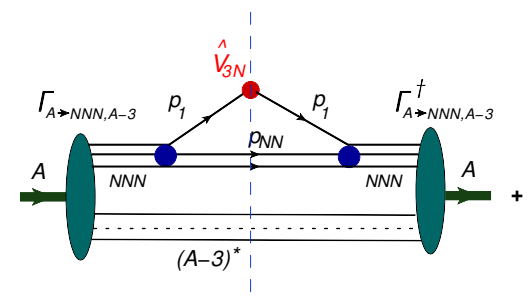

(c)

Figure 2.2: Expansion of the nuclear spectral function into the contributions of the mean field (a), the $2 \mathrm{~N}$ (b) and the $3 \mathrm{~N}$ (c) SRCs. For each case the initial nuclear transition vertices are different, corresponding to transition of $A \rightarrow N, A-1 ; A \rightarrow N N, A-1$ and $A \rightarrow N N N, A-3$ for the mean field, the $2 \mathrm{~N}$ and the $3 \mathrm{~N}$ SRCs respectively. The NN (b) and NNN (c) labels identify the $2 \mathrm{~N}$ and the $3 \mathrm{~N}$ SRCs with effective vertices elaborated in the text.

\subsubsection{Covariant amplitude for a nucleon in the nuclear mean field}

In the mean field approximation, the bound nucleon interacts with the nuclear mean field induced by the $A-1$ nuclear residual system. In such approximation, the nuclear spectral function corresponds to a configuration in which the residual nuclear system is identified as a coherent $A-1$ state with excitation energy in the order of tens of $\mathrm{MeV}$.

Applying the effective Feynman rules [68] to the diagram of Fig. 2.2(a) corresponding to the mean field contribution of nuclear spectral function, the following covariant amplitude is obtained:

$$
\begin{aligned}
\operatorname{Im} A^{M F}=\quad \operatorname{Im} & \int \chi_{A}^{s_{A}, \dagger} \Gamma_{A \rightarrow N, A-1}^{\dagger} \frac{\not p_{1}+M_{N}}{p_{1}^{2}-M_{N}^{2}} \hat{V}_{M F} \frac{\not p_{1}+M_{N}}{p_{1}^{2}-M_{N}^{2}}\left[\frac{G_{A-1}\left(p_{A-1}, \alpha\right)}{p_{A-1}^{2}-M_{A-1}^{2}+i \varepsilon}\right]^{o n} \\
& \times \Gamma_{A \rightarrow N, A-1} \chi_{A}^{s_{A}} \frac{d p_{A-1}^{0}}{i(2 \pi)} \frac{d^{3} \mathbf{p}_{A-1}}{(2 \pi)^{3}}
\end{aligned}
$$

where $M_{N}$ and $M_{A-1}$ are the masses of the nucleon and of the $A-1$ nuclear residual system respectively, $\chi_{A}$ is the nuclear spin wave function, $\Gamma_{A \rightarrow N, A-1}$ represents the covariant vertex of the $A \rightarrow N+(A-1)$ transition, $G_{A-1}$ describes the propagation of the $A-1$ nuclear residual system in the intermediate state having an excitation $\alpha$. Following the effective Feynman rules [68], the propagator of the form $\left(p_{A-1}^{2}-M_{A-1}^{2}+i \varepsilon\right)^{-1}$ and a factor $(2 \pi)^{-4}$ have been assigned to the spectator $A-1$. The label $[\cdots]^{o n}$ indicates that the cut diagram is estimated so that the 
residual nuclear system is on mass shell. The abbreviated notation $\not p_{1}=\gamma^{\mu} p_{\mu}$, where $\gamma^{\mu}$ are the Dirac $\gamma$ matrices, has been used [40]

\subsubsection{Covariant amplitude for two nucleons in short-range correlation}

For two-nucleons in short-range correlation, it is assumed that the intermediate nuclear state consists of two correlated fast $\left(>k_{F}\right)$ nucleons and a slow $\left(<k_{F}\right)$ coherent $A-2$ nuclear residual system.

Applying the effective Feynman rules [68] to the diagram of Fig. 2.2(b) corresponding to the $2 \mathrm{~N}$ SRC contribution of nuclear spectral function, the following covariant amplitude is obtained:

$$
\begin{aligned}
& \operatorname{Im} A^{2 N}= \\
& \quad \operatorname{Im} \int \chi_{A}^{s_{A}, \dagger} \Gamma_{A \rightarrow N N, A-2}^{\dagger} \frac{G\left(p_{N N}, s_{N N}\right)}{p_{N N}^{2}-M_{N N}^{2}} \Gamma_{N N \rightarrow N, N}^{\dagger} \frac{\not p_{1}+M_{N}}{p_{1}^{2}-M_{N}^{2}} \hat{V}_{2 N} \frac{\not p_{1}+M_{N}}{p_{1}^{2}-M_{N}^{2}}\left[\frac{\not p_{2}+M_{N}}{p_{2}^{2}-M_{N}^{2}+i \varepsilon}\right]^{o n} \\
& \quad \times \Gamma_{N N \rightarrow N, N} \frac{G\left(p_{N N}, s_{N N}\right)}{p_{N N}^{2}-M_{N N}^{2}}\left[\frac{G_{A-2}\left(p_{A-2}, s_{A-2}\right)}{p_{A-2}^{2}-M_{A-2}^{2}+i \varepsilon}\right]^{o n} \Gamma_{A \rightarrow N N, A-2} \chi_{A}^{s_{A}} \\
& \quad \times \frac{d p_{2}^{0}}{i(2 \pi)} \frac{d^{3} \mathbf{p}_{2}}{(2 \pi)^{3}} \frac{d p_{A-2}^{0}}{i(2 \pi)} \frac{d^{3} \mathbf{p}_{A-2}}{(2 \pi)^{3}},
\end{aligned}
$$

where $M_{N N}$ is the mass of the $2 \mathrm{~N}$ SRC system, $\Gamma_{A \rightarrow N N, A-2}$ now describes the transition of the nucleus A to the $N N$ SRC and coherent $A-2$ nuclear residual state, while the $\Gamma_{N N \rightarrow N, N}$ vertex describes the short range $N N$ interaction that generates two-nucleon correlation in the nuclear spectral function.

\subsubsection{Covariant amplitude for three nucleons in short-range correlation}

The nuclear spectral function that results from $3 \mathrm{~N}$ short-range correlations is described in Fig. 2.2(c) in which the intermediate state consists of three fast $\left(>k_{F}\right)$ nucleons and a slow $\left(<k_{F}\right)$ coherent $A-3$ nuclear residual system.

The dynamics of the 3N SRCs allow more complex interactions than that of the 2N SRCs. One of the complexities is the irreducible three-nucleon forces that can not be described by the $\mathrm{NN}$ interaction only. Such interactions may contain inelastic transitions such as the $N N \rightarrow N \Delta$ 
interaction. Some studies demonstrated [82] that irreducible three-nucleon forces predominantly contribute at very large magnitudes of missing energy characteristic to the $\Delta$ excitations $\sim 300 \mathrm{MeV} / c$. Thus for nuclear spectral functions for which the missing energy does not exceed the $\Delta$ resonance threshold $\sim M_{\Delta}-M_{N}$, only the contributions of the $N N \rightarrow N N$ interactions need to be considered.

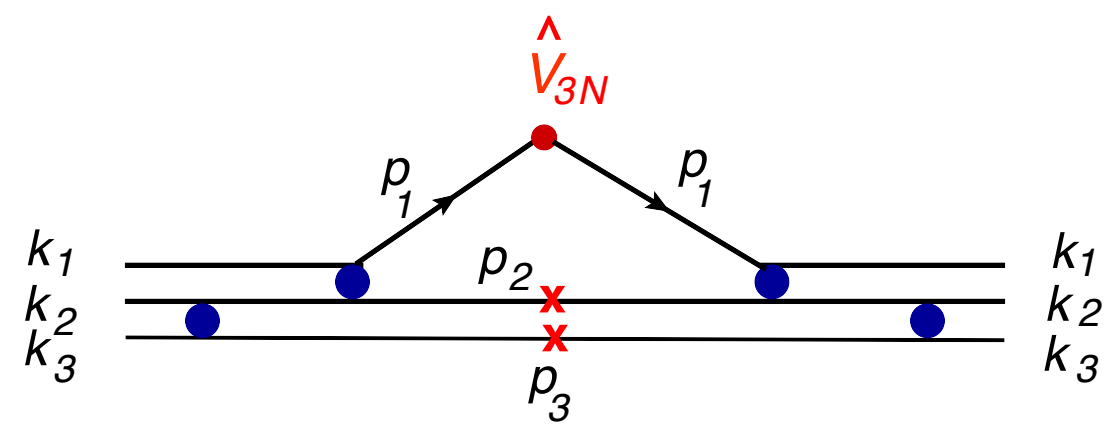

Figure 2.3: Diagram corresponding to the 3N SRC contribution to the nuclear spectral functions.

In the two sequential NN short-range interaction scenario for the generation of 3N SRCs, the spectral function can be represented through the diagram of Fig. 2.3, where it is assumed that the contribution of the low momentum $A-3$ nuclear residual system is negligible. The assumption results from the fact that a much larger momenta are involved in the $3 \mathrm{~N}$ SRCs as compared to the momenta in the $2 \mathrm{~N}$ SRCs discussed in the previous section. As a result the effects due to the center of mass motion of the $A-3$ system can be safely neglected.

In the collinear approximation, the initial three collinear nucleons undergo two shortrange NN interactions generating one nucleon with much larger momenta than the other two. It is assumed that the momentum fraction of the 3N SRCs carried by each initial nucleon is unity and that their total transverse momenta is neglected [see Eq. (2.113)]. Within the VN approximation, the collinear approximation assumes that the initial total momentum of the three nucleons is much smaller than $k_{s r c}$-momenta characteristic to NN SRC- and therefore can be neglected $\left(\mathbf{k}_{1}+\mathbf{k}_{2}+\mathbf{k}_{3}=0\right)$. The collinear approximation is commonly used in the calculation of the quark structure function of the nucleon in the valence quark region. Hence the calculations in the present dissertation for the LF approximation are analytically similar to the QCD calculation of the nucleon structure function. 
Applying the effective Feynman rules [68] to the diagram of Fig. 2.3 corresponding to the $3 \mathrm{~N}$ SRC contribution of the nuclear spectral function, the following covariant amplitude is obtained:

$$
\begin{aligned}
\operatorname{Im} A^{3 N}= & \operatorname{Im} \int \bar{u}\left(k_{1}, \lambda_{1}\right) \bar{u}\left(k_{2}, \lambda_{2}\right) \bar{u}\left(k_{3}, \lambda_{3}\right) \Gamma_{N N \rightarrow N, N}^{\dagger} \frac{\not p_{2^{\prime}}+M_{N}}{p_{2^{\prime}}^{2}-M_{N}^{2}} \Gamma_{N N \rightarrow N, N}^{\dagger} \frac{\not p_{1}+M_{N}}{p_{1}^{2}-M_{N}^{2}} \hat{V}_{3 N} \frac{\not p_{1}+M_{N}}{p_{1}^{2}-M_{N}^{2}} \\
& \times\left[\frac{\not p_{2}+M_{N}}{p_{2}^{2}-M_{N}^{2}+i \varepsilon}\right]^{o n} \Gamma_{N N \rightarrow N, N} \frac{\not p_{2^{\prime}}+M_{N}}{p_{2^{\prime}}^{2}-M_{N}^{2}}\left[\frac{\not p_{3}+M_{N}}{p_{3}^{2}-M_{N}^{2}+i \varepsilon}\right]^{o n} \Gamma_{N N \rightarrow N, N} \\
& \times u\left(k_{1}, \lambda_{1}\right) u\left(k_{2}, \lambda_{2}\right) u\left(k_{3}, \lambda_{3}\right) \frac{d p_{2}^{0}}{i(2 \pi)} \frac{d^{3} \mathbf{p}_{2}}{(2 \pi)^{3}} \frac{d p_{3}^{0}}{i(2 \pi)} \frac{d^{3} \mathbf{p}_{3}}{(2 \pi)^{4}}
\end{aligned}
$$

where " 2 " labels the intermediate state of the nucleon 2 after the first short-range NN interaction, $\lambda_{i}$ is the spin of the $i$ th nucleon and the $\Gamma_{N N \rightarrow N, N}$ is the same short range NN interaction vertex included in Eq. (2.10).

Note that there are several other 3N SRC diagrams which differ from that of Fig. 2.3 by the ordering of the two sequential NN short range interactions. In collinear approximation these diagrams result in the same analytic form both in VN and LF approximations (see, e.g., Ref. [125]), thus their contribution can be absorbed in the definition of the parameter $n_{3 N}^{N}$ [see Eq. (2.48)], which defines the contribution of the norm of the 3N SRCs to the total normalization of the nuclear spectral function.

\subsubsection{Models calculation of nuclear spectral functions}

The first step to calculate the nuclear spectral functions from the forward scattering amplitudes given by Eqs. (2.9)-(2.11) is to define the effective vertices $\hat{V}$ which identify the bound nucleon in the mean field, in the $2 \mathrm{~N}$ or in the $3 \mathrm{~N}$ SRCs, as well as to define the poles at which the cut propagators of the intermediate states are estimated. Both will depend on the approach used to deal with vacuum fluctuations, that is virtual nucleon or light-front approximation.

After the vertex definition, the effective Feynman rules [68] are applied to the covariant forward scattering amplitudes corresponding to the mean field, the two-, or the three-nucleon SRC contributions (Fig. 2.2) separately. Within the VN or the LF approximation, the loopintegrals are calculated through the on-mass shell conditions of intermediate states, by inte- 
gration through the positive poles of the cut propagators of the corresponding intermediate states. Finally, the numerators of the propagators are estimated by using sum rules representing the completeness relation for the wave functions of the intermediate states.

The following step is the definition of transition wave functions for the nucleus to nucleons and to nuclear residuals systems. Such definitions are formulated by the identification of the interaction diagrams for the bound states with the corresponding equations for the bound state wave function. For example in the non-relativistic limit, the interaction diagrams for the bound state, calculated based on the effective Feynman diagrammatic rules, are identified with the Lippmann-Schwinger equation $[27,30]$ in the non-relativistic limit. In the relativistic case, similar identifications are made with the Bethe-Salpeter type [12,37] (for VN approximation) or the Weinberg type [25] (for LF approximation) equations for the relativistic bound state wave function.

Finally, the mathematical models for the SRC nuclear spectral functions are simplified by introducing effective momentum distributions for $2 \mathrm{~N} \mathrm{SRC}$ which are function of the deuteron momentum distribution, as well as approximate Gaussian distribution models for the center of mass motion of the 2N SRC. For the mean field nuclear spectral functions, a nonrelativistic approximation obtained from the conventional mean field calculations for a single nucleon is applied.

\subsection{Nuclear spectral function in virtual nucleon approximation}

The main assumptions of the model for nuclear spectral function in virtual nucleon approximation are that the nucleus is in the laboratory frame, the interacting bound nucleon is described as a virtual particle, and the spectators are put on their mass-shells.

The nuclear spectral function, $S_{A}^{N}\left(\mathrm{p}, E_{m}\right)$, in virtual nucleon approximation is defined as the joint probability of finding a nucleon in the nucleus with momentum $\mathrm{p}$ and removal energy $E_{m}$. The conventional definition of the removal energy is

$$
E_{m}=E_{A-1}+M_{N}-M_{A}-\frac{\mathrm{p}^{2}}{2 M_{A-1}}
$$


where $E_{A-1}$ and $M_{A-1}$ are the energy and the mass of the $A-1$ residual nuclear system respectively, and the nonrelativistic expression for the kinetic energy of $A-1$ system is subtracted. However, in practice the kinetic energy of the $A-1$ system depends on the mean field, the $2 \mathrm{~N}-\mathrm{SRC}$ or the $3 \mathrm{~N}$ - SRC picture of the nuclear wave function, hence the corresponding kinetic energy will be accordingly defined for each particular case.

The following normalization condition for the nuclear spectral function in virtual nucleon approximation is defined with base on the conservation of baryonic number of the nucleus in hadron-nucleus scattering [41]:

$$
\sum_{N=1}^{A} \int S_{A}^{N}\left(\mathrm{p}, E_{m}\right) \alpha d^{3} \mathbf{p} d E_{m}=A
$$

where $\alpha$ is the ratio of the flux factors of the (external probe)-(bound nucleon) and (external probe)-(nucleus) systems, which in the high momentum limit of the probe (hadron or virtual photon) yields

$$
\alpha=\frac{E_{N}+\mathrm{p}_{z}}{M_{A} / A}=A \frac{p^{+}}{p_{A}^{+}}
$$

Here, $p^{+}$and $p_{A}^{+}$are the light-front longitudinal momenta of the bound nucleon and of the nucleus respectively, $E_{N}$ is the energy of the bound nucleon and the $z$ direction is defined opposite to the direction of the incoming probe.

Following the decomposition of Fig. 2.2, the mean field, the 2N, and the 3N SRC contributions to the nuclear spectral function are separately considered. In the VN approximation, the cut diagrams of Figs. 2.2 and 2.3 will be evaluated at the positive energy poles of the spectator residual system. For the mean field contribution, it corresponds to the positive energy pole of the coherent $A-1$ nuclear residual system. For the 2N SRC contribution, they correspond to the positive energy poles of the correlated nucleon and the $A-2$ nuclear residual system, whereas for the 3N SRC contribution, they correspond to the positive energy poles of the two correlated nucleons. 


\subsubsection{Nuclear spectral function in virtual nucleon approximation for a nucleon in the nuclear mean field}

In the mean-field approximation [Fig. 2.2(a)] the missing momentum $\mathbf{p}_{\mathbf{m}} \equiv-\mathbf{p}_{\mathbf{1}}$ and the missing energy $E_{m}$ characterizes the total momentum and the excitation energy of the $A-1$ residual nuclear system. In the nuclear shell model, $E_{m}$ also defines the energy needed to remove the nucleon from a particular nuclear shell. For such a situation, the energy and the momentum of the interacting nucleon can be recovered from the kinematic parameters of the on-shell spectator.

The calculation of the nuclear spectral function in virtual nucleon approximation for a nucleon in the mean field of the $A-1$ residual nuclear system, starts with the following definition of the effective vertex $\hat{V}_{M F}$ in the mean field covariant amplitude Eq.(2.9)

$$
\hat{V}_{M F}=i \bar{a}\left(p_{1}, s_{1}\right) \delta\left(\mathbf{p}_{1}+\mathbf{p}_{A-1}\right) \delta\left(E_{m}-E_{\alpha}\right) a\left(p_{1}, s_{1}\right)
$$

where $E_{\alpha}$ is the characteristic energy of the given nuclear shell, and the delta functions represent the momentum and the energy conservation in the vertex. The creation, $\bar{a}\left(p_{1}, s_{1}\right)$, and the annihilation, $a\left(p_{1}, s_{1}\right)$, operators in Dirac space are defined in such a way that they obey the following relation:

$$
a\left(p_{1}, s_{1}\right)\left(\not p_{1}+M_{N}\right)=\bar{u}\left(p_{1}, s_{1}\right) \text { and }\left(\not p_{1}+M_{N}\right) \bar{a}\left(p_{1}, s_{1}\right)=u\left(p_{1}, s_{1}\right)
$$

where $u\left(p_{1}, s_{1}\right)$ is the spinor of the virtual nucleon.

Since in the VN approximation the vacuum fluctuations are neglected, the integral in Eq. (2.9), needs only to be calculated through the positive energy pole of the propagator of the on-

shell $A-1$ residual nuclear system. Since this pole is displaced slightly below the real axis by the infinitesimal factor $i \varepsilon$, the integral by $d p_{A-1}^{0}$ is obtained by applying the Cauchy's residue theorem to the lowest-half complex plane semicircle enclosing the displaced pole in the positive 
sense (clockwise direction), namely

$$
\oint \frac{d p_{A-1}^{0}}{p_{A-1}^{2}-M_{A-1}^{2}+i \varepsilon}=\oint \frac{d p_{A-1}^{0}}{E_{A-1}^{2}-\left(M_{A-1}^{2}+\mathbf{p}_{A-1}^{2}-i \varepsilon\right)}=-\left.\frac{2 \pi i}{2 E_{A-1}}\right|_{E_{A-1}=\sqrt{M_{A-1}^{2}+\mathbf{p}_{A-1}^{2}}},
$$

where $p_{A-1}^{0}=E_{A-1}$.

Let $\chi_{A-1}$ be the the spin wave function of the on-shell $A-1$ residual nuclear system, then the numerator of the propagator in Eq. (2.9), can be expressed by the following sum rule representing the completeness relation for the wave function:

$$
G_{A-1}\left(p_{A-1}, \alpha\right)=\sum_{s_{A-1}} \chi_{A-1}\left(p_{A-1}, s_{A-1}, E_{\alpha}\right) \chi_{A-1}^{\dagger}\left(p_{A-1}, s_{A-1}, E_{\alpha}\right)
$$

It is important to note that in the relativistic treatment, the spin wave functions are momentum dependent as it is indicated in the argument of $\chi_{A-1}$. Such a momentum dependence is also accounted for the spin wave function of other particles discussed in the present dissertation.

The definition of the vertex (2.15) follows the convention (see, e.g., Ref. [62]) for which the annihilation operator projects the nucleon propagator to the positive energy state, and the nuclear transition vertex produces the Fock component of the nuclear wave function. Note that the above definition is different from the conventional definition (see e.g. Ref. [128]) in which the annihilation operator acts over the nuclear wave function to produce nucleon-hole states. However the final results in both approaches are similar in the nonrelativistic limit.

Hence, using the above convention and from Eqs. (2.16)- (2.18), the single nucleon wave function, $\psi_{N / A}$, for the given nuclear shell $E_{\alpha}$ is defined as

$$
\psi_{N / A}^{s_{A}}\left(p_{1}, s_{1}, p_{A-1}, s_{A-1}, E_{\alpha}\right)=\frac{\bar{u}\left(p_{1}, s_{1}\right) \chi_{A-1}^{\dagger}\left(p_{A-1}, s_{A-1}, E_{\alpha}\right) \Gamma_{A \rightarrow N, A-1} \chi_{A}^{s_{A}}}{\left(M_{N}^{2}-p_{1}^{2}\right) \sqrt{(2 \pi)^{3} 2 E_{A-1}}}
$$

Inserting the above wave function and the mean field vertex (2.15) into Eq.(2.9), and summing over all possible nuclear shells, $\alpha$, and spin projection, $s_{1}$ and $s_{A-1}$, gives the following expression for the nuclear spectral function in virtual nucleon approximation for a nucleon in the 
mean field

$$
S_{A, M F}^{N}\left(\mathrm{p}_{1}, E_{m}\right)=\sum_{\alpha} \sum_{s_{1}, s_{A-1}} \int\left|\psi_{N / A}^{s_{A}}\left(p_{1}, s_{1}, p_{A-1}, s_{A-1}, E_{\alpha}\right)\right|^{2} \delta\left(E_{m}-E_{\alpha}\right) \delta^{3}\left(\mathbf{p}_{1}+\mathbf{p}_{A-1}\right) d^{3} \mathbf{p}_{A-1},
$$

which defines the joint probability of finding a nucleon in the mean field of the nucleus with momentum $\mathrm{p}_{1}$ and removal energy $E_{m}$.

Integration of Eq. (2.20) over $d^{3} \mathbf{p}_{A-1}$ and through the delta function, $\delta^{3}\left(\mathbf{p}_{1}+\mathbf{p}_{A-1}\right)$, yields

$$
S_{A, M F}^{N}\left(\mathrm{p}_{1}, E_{m}\right)=\sum_{\alpha} \sum_{s_{1}, s_{A-1}}\left|\psi_{N / A}^{s_{A}}\left(p_{1}, s_{1}, p_{A-1}, s_{A-1}, E_{\alpha}\right)\right|^{2} \delta\left(E_{m}-E_{\alpha}\right)
$$

In order to obtain numerical estimates of the nuclear spectral function (2.21), it is important to consider that in the mean field approximation, the substantial strength of the wave function $\psi_{N / A}^{s_{A}}$ comes from the momentum range $\mathrm{p}_{1} \leq \mathrm{k}_{F}$. Hence, the nonrelativistic approximation is valid, so that the mean field wave function (2.19) can be approximated by the nonrelativistic wave function obtained from the conventional mean field calculations of the single nucleon wave functions.

Additionally, in the nonrelativistic limit $\alpha \approx 1+\frac{\mathrm{p}_{1, z}}{M_{A} / A}$, and, in Eq.(2.13), the $\frac{\mathrm{p}_{\mathrm{l}, z}}{M_{A} / A}$ part does not contribute to the integral, resulting in the condition for the nonrelativistic normalization:

$$
\int S_{A, M F}^{N}\left(\mathrm{p}, E_{m}\right) d E_{m} d^{3} \mathbf{p}=n_{M F}^{N}
$$

where $n_{M F}^{N}$ is the mean field contribution to the total normalization of the nuclear spectral function.

\subsubsection{Nuclear spectral function in virtual nucleon approximation for two nucleons in short-range correlation}

The calculation of the nuclear spectral function in virtual nucleon approximation for two nucleons in short-range correlation, starts with the definition of the removal energy $E_{m}^{2 N}$ for the $2 \mathrm{~N} \mathrm{SRC}$ in which the correlated NN pair has a total momentum $\mathbf{p}_{N N}=\mathbf{p}_{1}+\mathbf{p}_{2}=-\mathbf{p}_{A-2}$, in the 
mean field of the $A-2$ residual nuclear system. The magnitude of $E_{m}^{2 N}$ is therefore defined as

$$
E_{m}^{2 N}=E_{t h r}^{(2)}+T_{A-2}+T_{2}-T_{A-1}=E_{t h r}^{(2)}+\frac{\mathrm{p}_{A-2}^{2}}{2 M_{A-2}}+T_{2}-\frac{\mathrm{p}_{1}^{2}}{2 M_{A-1}},
$$

where $E_{t h r}^{(2)}$ is the threshold energy needed to remove two nucleons from the nucleus, with an approximated value of $E_{t h r}^{(2)} \approx 2 M_{N}+M_{A-2}-M_{A}$. Furthermore, $T_{A-1}$ and $T_{A-2}$ are the nonrelativistic kinetic energies of the $A-1$ and the $A-2$ residual nuclear systems respectively, and $T_{2}$ is the relativistic kinetic energy of the correlated nucleon 2. The expression for $T_{A-1}$ follows from the fact that in the rest frame of the nucleus $\mathbf{p}_{1}=-\mathbf{p}_{A-1}$, as well as from the definition of the removal energy in Eq. (2.12).

The effective vertex $\hat{V}_{2 N}$ in the 2N SRC covariant amplitude (2.10) is defined as

$$
\hat{V}_{2 N}=i \bar{a}\left(p_{1}, s_{1}\right) \delta^{3}\left(\mathbf{p}_{1}+\mathbf{p}_{2}+\mathbf{p}_{A-2}\right) \delta\left(E_{m}-E_{m}^{2 N}\right) a\left(p_{1}, s_{1}\right)
$$

where the creation, $\bar{a}\left(p_{1}, s_{1}\right)$, and the annihilation, $a\left(p_{1}, s_{1}\right)$, operators of the nucleon with fourmomentum $p_{1}$ and spin $s_{1}$ satisfy the relations of Eq. (2.16). The delta functions represent the momentum and energy conservation in the vertex.

Similarly to the propagator integration described in section 2.3.1, the integrations by $d p_{2}^{0}$ and $d p_{A-2}^{0}$ in Eq. (2.10) through the positive energy poles of the propagators of the on-shell particle 2, and $A-2$ nuclear residual system respectively, yields

$$
\begin{gathered}
\oint \frac{d p_{2}^{0}}{p_{2}^{2}-M_{N}^{2}+i \varepsilon}=\oint \frac{d p_{2}^{0}}{E_{2}^{2}-\left(M_{N}^{2}+\mathbf{p}_{2}^{2}-i \varepsilon\right)}=-\left.\frac{2 \pi i}{2 E_{2}}\right|_{E_{2}=\sqrt{M_{N}^{2}+\mathbf{p}_{2}^{2}}} \\
\oint \frac{d p_{A-2}^{0}}{p_{A-2}^{2}-M_{A-2}^{2}+i \varepsilon}=\oint \frac{d p_{A-2}^{0}}{E_{A-2}^{2}-\left(M_{A-2}^{2}+\mathbf{p}_{A-2}^{2}-i \varepsilon\right)}=-\left.\frac{2 \pi i}{2 E_{A-2}}\right|_{E_{A-2}=\sqrt{M_{A-2}^{2}+\mathbf{p}_{A-2}^{2}}}
\end{gathered}
$$

Let $\chi_{A-2}$ be the the spin wave function of the on-shell $A-2$ residual nuclear system, then the numerator of its propagator in Eq. (2.10), can be expressed by the following sum rule representing the completeness relation for the wave function:

$$
G\left(p_{A-2}, s_{A-2}\right)=\sum_{s_{A-2}} \chi_{A-2}\left(p_{A-2}, s_{A-2}\right) \chi_{A-2}^{\dagger}\left(p_{A-2}, s_{A-2}\right)
$$


where $s_{A-2}$ is the spin projection of the $A-2$ residual nuclear system. Similarly, the numerator of the propagator for the on-shell particle 2 is given by the completeness relation

$$
\not p_{2}+M_{N}=\sum_{s 2} u\left(p_{2}, s_{2}\right) \bar{u}\left(p_{2}, s_{2}\right)
$$

where $u\left(p_{2}, s_{2}\right)$ is the spinor of particle 2 with momentum $p_{2}$ and spin projection $s_{2}$ [40].

It is assumed that the center of mass momentum of the $2 \mathrm{~N} \mathrm{SRC} \mathrm{is} \mathrm{small} \mathrm{compared} \mathrm{to} \mathrm{the}$ relative motions of the nucleons in this type of correlation, then the numerator of its propagator in Eq. (2.10), can be expressed by the following sum rule representing the completeness relation for the wave function:

$$
G\left(p_{N N}, s_{N N}\right)=\sum_{s_{N N}} \chi_{N N}\left(p_{N N}, s_{N N}\right) \chi_{N N}^{\dagger}\left(p_{N N}, s_{N N}\right)
$$

where $\chi_{N N}$ is the spin wave function, and $s_{N N}$ is the projection of the total spin of the NN correlation with the three-momentum, $\mathbf{p}_{\mathrm{NN}}=-\mathbf{p}_{\mathrm{A}-2}$ in the rest frame of the nucleus.

Inserting Eqs. (2.16) and (2.25) -(2.28) in the covariant 2N SRC amplitude [Eq. (2.10)], and summing over all possible spin projections $s_{2}, s_{A-2}$ and $s_{N N}$, reduces the latter to the 2N SRC part of the nuclear spectral function in $\mathrm{VN}$ approximation, namely:

$$
\begin{aligned}
S_{A, 2 N}^{N}\left(\mathrm{p}_{1}, E_{m}\right)= & \sum_{s-1, s_{2}, s_{A-2}, s_{N N}, s_{N N}^{\prime}} \int \chi_{A}^{s_{A}, \dagger} \Gamma_{A \rightarrow N N, A-2}^{\dagger} \frac{\chi_{N N}\left(p_{N N}, s_{N N}\right) \chi_{N N}^{\dagger}\left(p_{N N}, s_{N N}\right)}{p_{N N}^{2}-M_{N N}^{2}} \Gamma_{N N \rightarrow N, N}^{\dagger} \\
& \times \frac{u\left(p_{1}, s_{1}\right)}{p_{1}^{2}-M_{N}^{2}} \delta^{3}\left(\mathbf{p}_{1}+\mathbf{p}_{2}+\mathbf{p}_{A-2}\right) \delta\left(E_{m}-E_{m}^{2 N}\right) \frac{\bar{u}\left(p_{1}, s_{1}\right)}{p_{1}^{2}-M_{N}^{2}} \\
& \times \frac{u\left(p_{2}, s_{2}\right) \bar{u}\left(p_{2}, s_{2}\right)}{2 E_{2}} \Gamma_{N N \rightarrow N, N} \frac{\chi_{N N}\left(p_{N N}, s_{N N}\right) \chi_{N N}^{\dagger}\left(p_{N N}, s_{N N}\right)}{p_{N N}^{2}-M_{N N}^{2}} \\
& \times \frac{\chi_{A-2}\left(p_{A-2}, s_{A-2}\right) \chi_{A-2}^{\dagger}\left(p_{A-2}, s_{A-2}\right)}{2 E_{A-2}} \Gamma_{A \rightarrow N N, A-2} \chi_{A}^{s_{A}} \frac{d^{3} \mathbf{p}_{2}}{(2 \pi)^{3}} \frac{d^{3} \mathbf{p}_{A-2}}{(2 \pi)^{3}},
\end{aligned}
$$

which defines the joint probability of finding a nucleon in NN SRC with momentum $\mathrm{p}_{1}$ and removal energy $E_{m}$.

The introduction of the transition wave function of the center of mass of the $2 \mathrm{~N}$ SRC Eq. (2.28), implies the decoupling of the slow moving center of mass of the $2 \mathrm{~N}$ SRC from the fast 
moving relative motion of the nucleons in the correlation. Then, the $2 \mathrm{~N}$ SRC center of mass wave function for the transition $A \rightarrow(N N)+(A-2)$ in the rest frame of the nucleus is defined as

$$
\psi_{C M}^{s_{A}}\left(p_{N N}, s_{N N}, p_{A-2}, s_{A-2}\right)=\frac{\chi_{N N}^{\dagger}\left(p_{N N}, s_{N N}\right) \chi_{A-2}^{\dagger}\left(p_{A-2}, s_{A-2}\right) \Gamma_{A \rightarrow N N, A-2} \chi_{A}^{s_{A}}}{\left(M_{N N}^{2}-p_{N N}^{2}\right) \sqrt{2 E_{A-2}(2 \pi)^{3}}},
$$

and the wave function for the transition $(N N) \rightarrow N+N$, that represents the relative motion of nucleons in 2N SRC in the rest frame of the 2N SRC (see e.g. Ref. [68]), is defined as

$$
\psi_{N N}^{s_{N N}}\left(p_{1}, s_{1}, p_{2}, s_{2}\right)=\frac{\bar{u}\left(p_{1}, s_{1}\right) \bar{u}\left(p_{2}, s_{2}\right) \Gamma_{N N \rightarrow N, N} \chi_{N N}\left(p_{N N}, s_{N N}\right)}{\left(M_{N}^{2}-p_{1}^{2}\right) \sqrt{2 E_{2}(2 \pi)^{3}}} .
$$

inserting the above defined wave functions in the 2N SRC nuclear spectral function (2.29) yields

$$
\begin{aligned}
S_{A, 2 N}^{N}\left(\mathrm{p}_{1}, E_{m}\right)= & \sum_{\substack{s_{1}, s_{2}, s_{A-2}, s_{N N}, s_{N N}^{\prime} \\
s_{A}}} \int \psi_{C M}^{s_{A}, \dagger}\left(p_{N N}, s_{N N}^{\prime}, s_{A-2}\right) \psi_{N N}^{s_{N N}^{\prime}, \dagger}\left(p_{1}, s_{1}, p_{2}, s_{2}\right) \\
& \times \delta^{3}\left(\mathbf{p}_{1}+\mathbf{p}_{2}-\mathbf{p}_{N N}\right) \delta\left(E_{m}-E_{m}^{2 N}\right) d^{3} \mathbf{p}_{2} d^{3} \mathbf{p}_{N N}
\end{aligned}
$$

where the relation $\mathbf{p}_{\mathrm{NN}}=-\mathbf{p}_{\mathrm{A}-2}$, in the nucleus rest frame, has been used.

The nuclear spectral function (2.32) can be integrated by $d^{3} \mathbf{p}_{2}$ through the $\delta^{3}\left(\mathbf{p}_{1}+\mathbf{p}_{2}-\right.$ $\mathbf{p}_{N N}$ ) delta function. Furthermore using the 2N SRC model in which the wave function of the relative motion is dominated by the $p n$ component with spin equal to 1 , and with the low momentum CM wave function being in the S state, the summation by $s_{A-2}$ results in $\delta_{s_{N N}, s_{N N}^{\prime}}$. Then, the 2N SRC nuclear spectral function (2.32) can be expressed as

$$
\begin{array}{r}
S_{A, 2 N}^{N}\left(\mathrm{p}_{1}, E_{m}\right)=\sum_{s_{1}, s_{2}, s_{A-2}, s_{N N}} \int\left|\psi_{C M}^{s_{A}}\left(p_{N N}, s_{N N}, s_{A-2}\right)\right|^{2}\left|\psi_{N N}^{s_{N N}}\left(p_{1}, s_{1}, p_{2}, s_{2}\right)\right|^{2} \\
\times \delta\left(E_{m}-E_{m}^{2 N}\right) d^{3} \mathbf{p}_{N N},
\end{array}
$$

where $\mathbf{p}_{\mathrm{NN}}=\mathbf{p}_{2}+\mathbf{p}_{\mathbf{1}}$.

The above expression can be represented in a more simple form by noticing that the $2 \mathrm{~N}$ SRC center of mass wave function (2.30) depends on the center of mass momentum, and that 
the wave function for the relative motion of nucleons in 2N SRC depends on their relative momentum. Thus, by introducing effective momentum distribution of the 2N SRC center of mass, $n_{C M}$, as well for the relative momentum of the nucleons in $2 \mathrm{~N}$ SRC, $n_{N N}$, and by summing over the final and averaging all possible initial polarization configurations in (2.33), the following expression is obtained for the $2 \mathrm{~N}$ SRC nuclear spectral function

$$
S_{A, 2 N}^{N}\left(\mathrm{p}_{1}, E_{m}\right)=\int n_{C M}\left(\mathrm{p}_{N N}\right) n_{N N}\left(\mathrm{p}_{r e l}\right) \delta\left(E_{m}-E_{m}^{2 N}\right) d^{3} \mathbf{p}_{N N}
$$

where $\mathbf{p}_{\text {rel }}=\frac{\mathbf{p}_{1}-\mathbf{p}_{2}}{2}$.

The normalization of the 2N SRC nuclear spectral function (2.34) should be related to the total probability of finding a nucleon in such a correlation, and can be defined from the normalization condition of Eq. (2.13), namely:

$$
\int S_{A, 2 N}^{N}\left(\mathrm{p}_{1}, E_{m}\right) \alpha_{1} d E_{m} d^{3} \mathbf{p}_{1}=n_{2 N}^{N}
$$

where for the 2N SRC model $\alpha \equiv \alpha_{1}=\frac{M_{N}-E_{m}-T_{A-1}+\mathrm{p}_{1, z}}{M_{A} / A}$, and $n_{2 N}^{N}$ is the contribution to the total normalization of the nuclear spectral function.

If the relative and the center of mass momentum distributions of the NN correlations are given, the 2N SRC part of the nuclear spectral function (2.34) can be numerically calculated. Then, since it is assumed that the center of mass momenta of the NN SRCs are small, $n_{C M}$ is approximated by the distribution obtained in Ref. [52] through the overlap of two Fermi momentum distributions which results in the simple Gaussian distribution:

$$
n_{C M}\left(\mathrm{p}_{N N}\right)=N_{0}(A) e^{-\beta(A) \mathrm{p}_{N N}^{2}}
$$

normalized to unity: $\int n_{C M}\left(p_{N N}\right) d^{3} \mathbf{p}_{N N}=1$. The parameter $\beta(A)$ is estimated from the nuclear mean field distribution, while $N_{0}(A)$ is found from the normalization condition.

The relative momentum distribution of the NN SRC, $n_{N N}\left(\mathrm{p}_{r e l}\right)$, can be modeled according to Ref. [104, 112], where the high momentum strength of the nucleon momentum distribution is predicted to be inverse proportional to the relative fraction of the nucleon in the 
nucleus. Such a distribution is in agreement with the recently observed dominance of $p n$ SRCs $[85,90,119]$ and can be expressed in the form:

$$
n_{N N}^{N}\left(\mathrm{p}_{r e l}\right)=\frac{a_{2}(A, Z)}{\left(2 x_{N}\right)^{\gamma}} \frac{n_{d}\left(\mathrm{p}_{r e l}\right) \Theta\left(\mathrm{p}_{r e l}-\mathrm{k}_{s r c}\right)}{\frac{M_{N}-E_{m}-T_{A-1}}{M_{A} / A}}
$$

where $\Theta$ is the step function; $x_{N}=N / A$ is the nucleon relative fraction [112], with $N$ being the number of protons $(Z)$ and neutrons $(A-Z)$ in the nucleus $A$; the parameter $a_{2}(A, Z)$ is related to the probability of finding $2 \mathrm{~N}$ SRC in the nucleus, $A$, relative to the deuteron; and $\gamma$ is a free parameter $\gamma \lesssim 1 . n_{d}\left(\mathrm{p}_{r e l}\right)$ is the high momentum distribution in the deuteron, and $k_{s r c} \gtrsim k_{F}$ is the momentum threshold at which a NN system with such relative momentum can be considered in short-range correlation. The factor $\frac{M_{N}-E_{m}-T_{A-1}}{M_{A} / A}$ is the generalization of the normalization scheme of [41] which enforces the normalization condition of Eq. (2.13). The normalization of the above defined distribution, $\int n_{N N}^{N}(p) d^{3} \mathbf{p}=n_{2 N}^{N}$, defines the contribution of the $2 \mathrm{~N}$ SRCs to the total norm of the momentum distribution for the nucleon $N$.

The 2N SRC nuclear spectral function in VN approximation [Eq.(2.34)], together with the momentum distribution for the NN SRC center of mass [Eq.(2.36)], the relative momentum distribution of the NN SRC [Eq.(2.37)], and the normalization condition [Eq.(2.35)], constitute the mathematical model from which the corresponding computational models and numerical estimates are obtained in the chapters 3 and 4 of the present dissertation.

It is worth mentioning that in the non-relativistic limit, and assuming an equal 2N SRC contributions from proton and neutron: $n_{N N}^{N}\left(\mathrm{p}_{r e l}\right)=a_{2}(A) n_{d}\left(\mathrm{p}_{r e l}\right)$, the $2 \mathrm{~N}$ SRC nuclear spectral function [Eq.(2.34)] reduces to the "NN SRC-CM motion" model of Ciofi-Simula [47, 52].

\subsubsection{Nuclear spectral function in virtual nucleon approximation for three nucle- ons in short-range correlation}

The calculation of the nuclear spectral function in virtual nucleon approximation for three nucleons in short-range correlation, starts with the definition of the magnitude of the removal energy $E_{m}^{3 N}$ which is calculated considering the 3N SRC model in which the recoil nuclear system consists of two fast nucleons and a slow $A-3$ residual nuclear system whose excitation 
energy is neglected. The magnitude of $E_{m}^{3 N}$ is therefore defined as

$$
E_{m}^{3 N}=E_{t h r}^{(2)}+T_{2}+T_{3}-T_{A-1}=E_{t h r}^{(3)}+T_{3}+T_{2}-\frac{\mathrm{p}_{1}^{2}}{2 M_{A-1}}
$$

where $E_{t h r}^{(3)}$ is the threshold energy needed to remove three nucleons from the nucleus, with an approximated value of $E_{t h r}^{(3)} \approx 3 M_{N}+M_{A-3}-M_{A}$ where $M_{A-3}$ is the mass of the $A-3$ residual nuclear system. Within the considered 3N SRC model, the kinetic energy of the $A-1$ residual nuclear system is the result of the kinetic energies of the correlated spectator nucleons, $T_{2}$ and $T_{3}$ which are treated relativistically. Here, as in the case of $2 \mathrm{~N} \mathrm{SRC,} \mathrm{the} \mathrm{expression} \mathrm{for} \mathrm{the} \mathrm{kinetic}$ energy $T_{A-1}$ follows from the fact that in the rest frame of the nucleus $\mathbf{p}_{1}=-\mathbf{p}_{A-1}$, as well as from the definition of the missing energy in Eq. (2.12).

For the $3 \mathrm{~N}$ SRC model in collinear approximation, the effective vertex $\hat{V}_{3 N}$ in the $3 \mathrm{~N}$ SRC covariant amplitude Eq. (2.11) is defined as

$$
\hat{V}_{3 N}=i \bar{a}\left(p_{1}, s_{1}\right) \delta^{3}\left(\mathbf{p}_{1}+\mathbf{p}_{2}+\mathbf{p}_{3}\right) \delta\left(E_{m}-E_{m}^{3 N}\right) a\left(p_{1}, s_{1}\right),
$$

where the creation, $\bar{a}\left(p_{1}, s_{1}\right)$, and the annihilation, $a\left(p_{1}, s_{1}\right)$, operators of a nucleon with fourmomentum $p_{1}$ and spin $s_{1}$ satisfy the relations of Eq. (2.16). The delta functions represent the momentum and energy conservation in the vertex.

Similar to the propagator integration described in section 2.3.2, the integrations by $d p_{2}^{0}$ and $d p_{3}^{0}$ in Eq. (2.11), are done through the positive energy poles of the propagators of the on-shell particles 2 and 3 yielding:

$$
\begin{aligned}
& \oint \frac{d p_{2}^{0}}{p_{2}^{2}-M_{N}^{2}+i \varepsilon}=\oint \frac{d p_{2}^{0}}{E_{2}^{2}-\left(M_{N}^{2}+\mathbf{p}_{2}^{2}-i \varepsilon\right)}=-\left.\frac{2 \pi i}{2 E_{2}}\right|_{E_{2}=\sqrt{M_{N}^{2}+\mathbf{p}_{2}^{2}}} \\
& \oint \frac{d p_{3}^{0}}{p_{3}^{2}-M_{N}^{2}+i \varepsilon}=\oint \frac{d p_{3}^{0}}{E_{3}^{2}-\left(M_{N}^{2}+\mathbf{p}_{3}^{2}-i \varepsilon\right)}=-\left.\frac{2 \pi i}{2 E_{3}}\right|_{E_{3}=\sqrt{M_{N}^{2}+\mathbf{p}_{3}^{2}}}
\end{aligned}
$$

The numerator of the propagator for the on-shell particle $i$ is defined, as in the 2N SRC case, by

$$
\not p_{i}+M_{N}=\sum_{s i} u\left(p_{i}, s_{i}\right) \bar{u}\left(p_{i}, s_{i}\right)
$$


where $u\left(p_{i}, s_{i}\right)$ is the spinor of particle $i$ with momentum $p_{i}$ and spin projection $s_{i}$, for $i=2,2^{\prime}, 3$, [40].

Inserting Eqs. (2.16), (2.40), and (2.41) in the covariant 3N SRC amplitude [Eq. (2.11)], and summing over all possible spin projections $s_{2}, s_{2^{\prime}}$ and $s_{3}$, reduces the latter to the 3N SRC part of the nuclear spectral function, namely:

$$
\begin{aligned}
& S_{A, 3 N}^{N}\left(\mathrm{p}_{1}, E_{m}\right)=\sum_{s_{2^{\prime}}, \tilde{s}_{2^{\prime}}, s_{2}, s_{3}} \int \bar{u}\left(k_{1}, \lambda_{1}\right) \bar{u}\left(k_{2}, \lambda_{2}\right) \bar{u}\left(k_{3}, \lambda_{3}\right) \Gamma_{N N \rightarrow N, N}^{\dagger} \frac{u\left(p_{2^{\prime}}, s_{2^{\prime}}\right) \bar{u}\left(p_{2^{\prime}}, s_{2^{\prime}}\right)}{p_{2^{\prime}}^{2}-M_{N}^{2}} \\
& \quad \times \Gamma_{N N \rightarrow N, N}^{\dagger} \frac{u\left(p_{1}, s_{1}\right)}{p_{1}^{2}-M_{N}^{2}} \delta^{3}\left(\mathbf{p}_{1}+\mathbf{p}_{2}+\mathbf{p}_{3}\right) \delta\left(E_{m}-E_{m}^{3 N}\right) \frac{\bar{u}\left(p_{1}, s_{1}\right)}{p_{1}^{2}-M_{N}^{2}} \\
& \quad \times \frac{u\left(p_{2}, s_{2}\right) \bar{u}\left(p_{2}, s_{2}\right)}{2 E_{2}} \Gamma_{N N \rightarrow N, N} \frac{u\left(p_{2^{\prime}}, \tilde{s}_{2^{\prime}}\right) \bar{u}\left(p_{2^{\prime}}, \tilde{s}_{2^{\prime}}\right)}{p_{2^{\prime}}^{2}-M_{N}^{2}} \frac{u\left(p_{3}, s_{3}\right) \bar{u}\left(p_{3}, s_{3}\right)}{2 E_{3}} \\
& \quad \times \Gamma_{N N \rightarrow N, N} u\left(k_{1}, \lambda_{1}\right) u\left(k_{2}, \lambda_{2}\right) u\left(k_{3}, \lambda_{3}\right) \frac{d^{3} \mathbf{p}_{3}}{(2 \pi)^{3}} \frac{d^{3} \mathbf{p}_{2}}{(2 \pi)^{3}}
\end{aligned}
$$

which defines the joint probability of finding a nucleon in $3 \mathrm{~N}$ SRC with momentum $\mathrm{p}_{1}$ and removal energy $E_{m}$.

In analogy with Eq. (2.31), the following $2 N$ SRC wave functions can be introduced for the nuclear spectral function (2.42), as follows:

$$
\begin{gathered}
\psi_{N N}\left(p_{2^{\prime}}, s_{2^{\prime}} ; p_{3}, s_{3} ; k_{2}, \lambda_{2}, k_{3}, \lambda_{3}\right)=\frac{\bar{u}\left(p_{2^{\prime}}, s_{2^{\prime}}\right) \bar{u}\left(p_{3}, s_{3}\right) \Gamma_{N N \rightarrow N, N} u\left(k_{2}, \lambda_{2}\right) u\left(k_{3}, \lambda_{3}\right)}{\left(M_{N}^{2}-p_{2^{\prime}}^{2}\right) \sqrt{2 E_{3}(2 \pi)^{3}}}, \\
\psi_{N N}\left(p_{1}, s_{1} ; p_{2}, s_{2} ; k_{1}, \lambda_{1}, p_{2^{\prime}}, s_{2^{\prime}}\right)=\frac{\bar{u}\left(p_{1}, s_{1}\right) \bar{u}\left(p_{2}, s_{2}\right) \Gamma_{N N \rightarrow N, N} u\left(k_{1}, \lambda_{1}\right) u\left(p_{2^{\prime}}, s_{2^{\prime}}\right)}{\left(M_{N}^{2}-p_{1}^{2}\right) \sqrt{2 E_{2}(2 \pi)^{3}}},
\end{gathered}
$$

where the wave function (2.43) represents the first 2N SRC, shown in Fig. 2.3, between particle 2 with momentum $k_{2}$, and particle 3 with momentum $k_{3}$, which results in particle $2^{\prime}$ with momentum $p_{2^{\prime}}$, and particle 3 with momentum $p_{3}$. Whereas the wave function (2.44) represents the second 2N SRC between particle 1 with momentum $k_{1}$, and particle $2^{\prime}$ with momentum $p_{2}^{\prime}$, which results in particle 1 with momentum $p_{1}$, and particle 2 with momentum $p_{2}$. 
Inserting Eqs. (2.43) and (2.44) in Eq.(2.42), the 3N SRC nuclear spectral function can be expressed as follows:

$$
\begin{aligned}
S_{A, 3 N}^{N}\left(\mathrm{p}_{1}, E_{m}\right)= & \sum_{\substack{s_{2^{\prime}}, \tilde{s}_{2^{\prime}}, s_{2}, s_{3}\\
}} \int \psi_{N N}^{\dagger}\left(p_{1}, s_{1}, p_{2}, s_{2} ; k_{1}, \lambda_{1}, p_{2^{\prime}}, s_{2^{\prime}}\right) \psi_{N N}^{\dagger}\left(p_{2^{\prime}}, s_{2^{\prime}}, p_{3}, s_{3} ; k_{2}, \lambda_{2}, k_{3}, \lambda_{3}\right) \\
& \left.\times \delta^{3}\left(\mathbf{p}_{1}+\mathbf{p}_{2}+\mathbf{p}_{3}\right) \delta\left(E_{m}-E_{m}, s_{2} ; k_{1}, \lambda_{1}, p_{2^{\prime}}, s_{2^{\prime}}\right) \psi_{N N}{ }^{3} \mathbf{p}_{3} d^{3} p_{2^{\prime}}, \tilde{s}_{2^{\prime}} ; p_{3}, s_{3} ; k_{2}, \lambda_{2}, k_{3}, \lambda_{3}\right)
\end{aligned}
$$

The nuclear spectral function (2.45) can be integrated by $d^{3} \mathbf{p}_{2}$ through the $\delta^{3}\left(\mathbf{p}_{1}+\mathbf{p}_{2}+\right.$ $\mathbf{p}_{3}$ ) delta function. Furthermore, using the 2N SRC model in which the wave function of the relative motion is dominated by the $p n$ component with spin equal to 1 , the summation over the polarizations of 2 and 3 particles, $s_{2}$ and $s_{3}$, results in in $s_{2}=s_{2^{\prime}}=\tilde{s}_{2^{\prime}}$. Then, the 3N SRC nuclear spectral function (2.45) can be expressed as

$$
\begin{aligned}
S_{A, 3 N}^{N}\left(\mathrm{p}_{1}, E_{m}\right)= & \sum_{s_{1}, s_{2}, s_{3}} \int\left|\psi_{N N}\left(p_{2^{\prime}}, s_{2}, p_{3}, s_{3} ; k_{2}, \lambda_{2}, k_{3}, \lambda_{3}\right)\right|^{2} \\
& \times\left|\psi_{N N}\left(p_{1}, s_{1} ; p_{2}, s_{2} ; k_{1}, \lambda_{1}, p_{2^{\prime}}, s_{2}\right)\right|^{2} \delta\left(E_{m}-E_{m}^{3 N}\right) d^{3} \mathbf{p}_{3} .
\end{aligned}
$$

The above expression can be represented in a more simple form by noticing that the wave function (2.43), for the first $2 \mathrm{~N} \mathrm{SRC}$, depends on the relative momentum of particles $2^{\prime}$ and

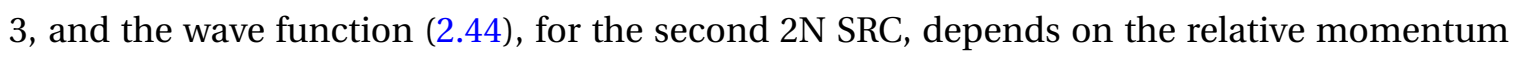
of particles 1 and 2. Thus, by introducing effective relative momentum distribution for the nucleons in the first and second 2N SRC, $n_{N N}$, and by summing over the final and averaging all possible initial polarization configurations in (2.46), the following simplified expression is obtained for the 3N SRC nuclear spectral function

$$
S_{A, 3 N}^{N}\left(\mathrm{p}_{1}, E_{m}\right)=\int n_{N N}\left(\mathrm{p}_{2^{\prime}, 3}\right) n_{N N}\left(\mathrm{p}_{12}\right) \delta\left(E_{m}-E_{m}^{3 N}\right) d^{3} \mathbf{p}_{3},
$$

where in the collinear approximation: $\mathbf{p}_{\mathbf{1 2}}=\frac{\mathbf{p}_{\mathbf{1}}-\mathbf{p}_{\mathbf{2}}}{2}=\mathbf{p}_{\mathbf{1}}+\frac{\mathbf{p}_{\mathbf{3}}}{2}$ and $\mathbf{p}_{2^{\prime}, \mathbf{3}}=\frac{\mathbf{p}_{\mathbf{2}^{\prime}}-\mathbf{p}_{\mathbf{3}}}{2} \approx \frac{-\mathbf{p}_{3}-\mathbf{p}_{3}}{2} \approx-\mathbf{p}_{\mathbf{3}}$.

The normalization of the 3N SRC nuclear spectral function (2.47) should be related to the total probability of finding a nucleon in such a correlation, and can be defined from the 
normalization condition of Eq. (2.13) which yields:

$$
\int S_{A, 3 N}^{N}\left(\mathrm{p}_{1}, E_{m}\right) \alpha_{1} d^{3} \mathbf{p}_{1} d E_{m}=n_{3 N}^{N}
$$

where $\alpha$ was defined in Eq. (2.35), and $n_{3 N}^{N}$ is the contribution to the total normalization of the nuclear spectral function.

Within the model of $p n$ dominance of two-nucleon SRCs, the 3N SRCs are predicted to be generated predominantly through two sequential short-range $p n$ interactions. Then, the overall probability of finding such correlations is predicted to be proportional to the factor $a_{2}^{2}(A, Z)$, where $a_{2}(A, Z)$ is defined in Eq. (2.37). Then, by using relations similar to those defined in Eq. (2.37), the following approximation is obtained for the product of the relative momentum distributions in Eq. (2.47)

$$
n_{N N}\left(\mathrm{p}_{2^{\prime}, 3}\right) n_{N N}\left(\mathrm{p}_{12}\right)=a_{2}^{2}(A, Z) C^{N}(A, Z) \frac{n_{d}\left(\mathrm{p}_{2^{\prime}, 3}\right) n_{d}\left(\mathrm{p}_{12}\right)}{\frac{M_{N}-E_{m}-T_{A-1}}{M_{A} / A}} \Theta\left(\mathrm{p}_{2^{\prime}, 3}-\mathrm{k}_{s r c}\right) \Theta\left(\mathrm{p}_{12}-\mathrm{k}_{s r c}\right)
$$

where $\mathrm{k}_{s r c}>\mathrm{k}_{F}$ is the relative momentum threshold at which the $N N$ system can be considered in short-range correlation. Here $C^{N}(A, Z)$ is a suppression factor which accounts for the suppression of the $3 \mathrm{~N}$ configurations with two identical spectators like $p p$ and $n n$ pairs, that is, effects associated with the isospin structure of two-nucleon recoil system. Namely, in the collinear approximation two recoil nucleons emerge with small relative momenta (or invariant mass). In Ref. [82] it was demonstrated that the $N N$ system with small relative momenta is strongly dominated in the isosinglet $p n$ channel. The dominance introduces an additional restriction on the isospin composition of the $3 \mathrm{~N}$ SRCs, in which the recoil $N N$ system predominately consists of a $p n$ pair. For example, one direct consequence of such dynamics is that high momentum neutrons in ${ }^{3} \mathrm{He}$ nucleus can not be generated in 3N SRCs while protons can.

The 3N SRC nuclear spectral function in VN approximation [Eq.(2.47)], together with the product of momentum distribution of the first and second NN SRC [Eq.(2.49)], and the normalization condition [Eq.(2.48)], constitute the mathematical model from which the correspond- 
ing computational models and numerical estimates are obtained in the chapters 3 and 4 of the present dissertation.

\subsection{Nuclear spectral function in light-front approximation}

The nuclear spectral function on the light-front (LF) was for the first time defined in Ref. [43], however its calculation from first principles is impossible due to the lack of knowledge of the LF nuclear wave functions. In the present dissertation, two assumptions are formulated to calculate the LF nuclear spectral functions. The first assumption is that the nuclear mean field contribution to the LF nuclear spectral function corresponds to the nonrelativistic limit of the momentum and missing energy of a bound nucleon. As a result the mean field part of the LF nuclear spectral function can be related to the mean field contribution of the conventional nuclear spectral functions as discussed in section 2.3.1. The second assumption is that the dynamics of the LF nuclear spectral function in the high momentum domain is mainly defined by the $p n$ interaction at short distances. Thus, a model for the LF deuteron wave function at short distances is sufficient to obtain the models for the $2 \mathrm{~N}$ and the $3 \mathrm{~N}$ SRC LF nuclear spectral functions.

The first step to obtain the LF nuclear spectral functions is the definition of the kinematic parameters that characterize the bound nucleon in the light front, as well as the sum rules that the LF nuclear spectral functions should satisfy.

In defining the LF nuclear spectral function the primary requirement is that it should be a Lorentz boost invariant function in the direction of the large CM momentum of the nucleus $p_{A}$. To satisfy this condition, the bound nucleon $N$ must be described by a light-front "+" momentum fraction $\alpha_{N}=A \frac{p_{N}^{+}}{p_{A}^{+}}$, transverse (to $\mathbf{p}_{\mathbf{A}}$ ) momentum $\mathbf{p}_{\mathbf{N}, \perp}$ and an invariant mass $\tilde{M}_{N}^{2}=p_{N}^{-} p_{N}^{+}-\mathbf{p}_{N, \perp}^{2}$.

For future derivations, it is useful to present the invariant phase space of bound nucleon,

$d^{4} p_{N}$, as a function of the light-front variables $\left(\alpha_{N}, \mathbf{p}_{N, \perp}, \tilde{M}_{N}^{2}\right)$. Thus, to calculate the Jacobian 
for the change of variables $\left(\mathrm{p}_{\mathrm{N}}^{+}, \mathrm{p}_{\mathrm{N}}^{-}, \mathbf{p}_{N, \perp}\right)$ to $\left(\alpha_{N}, \tilde{M}_{N}^{2}, \mathbf{p}_{N, \perp}\right)$ the following derivatives are used

$$
\frac{\partial \alpha_{N}}{\partial \mathrm{p}_{\mathrm{N}}^{+}}=\frac{A}{\mathrm{p}_{\mathrm{A}}^{+}}, \quad \frac{\partial \alpha_{N}}{\partial \mathrm{p}_{\mathrm{N}}^{-}}=0, \quad \frac{\partial \tilde{\mathrm{M}}_{\mathrm{N}}^{2}}{\partial \mathrm{p}_{\mathrm{N}}^{+}}=\mathrm{p}_{\mathrm{N}}^{-}, \quad \frac{\partial \tilde{M}_{N}^{2}}{\partial \mathrm{p}_{\mathrm{N}}^{-}}=\mathrm{p}_{\mathrm{N}}^{+},
$$

hence, the Jacobian is given by

$$
J=\left(\frac{\partial \alpha_{N}}{\partial \mathrm{p}_{\mathrm{N}}^{+}} \frac{\partial \tilde{\mathrm{M}}_{\mathrm{N}}^{2}}{\partial \mathrm{p}_{\mathrm{N}}^{-}}-\frac{\partial \alpha_{N}}{\partial \mathrm{p}_{\mathrm{N}}^{+}} \frac{\partial \tilde{\mathrm{M}}_{\mathrm{N}}^{2}}{\partial \mathrm{p}_{\mathrm{N}}^{-}}\right)^{-1}=\left(\frac{A \mathrm{p}_{\mathrm{N}}^{+}}{\mathrm{p}_{\mathrm{A}}^{+}}\right)^{-1}=\frac{1}{\alpha_{N}} .
$$

Then, the invariant phase space $d^{4} p_{N}$ in the light-front can be expressed as follows:

$$
d^{4} p_{N}=\frac{1}{2} d \mathrm{p}_{N}^{-} d \mathrm{p}_{N}^{+} d \mathbf{p}_{N \perp}=\frac{d \alpha_{N}}{2 \alpha_{N}} d \mathbf{p}_{N \perp} d \tilde{M}_{N}^{2}
$$

After identifying the kinematic variables describing the bound nucleon, the LF nuclear spectral function, $P_{A}\left(\alpha_{N}, p_{N, \perp}, \tilde{M}_{N}^{2}\right)$, is defined as the joint probability of finding a bound nucleon in the nucleus with light-front momentum fraction $\alpha_{N}$, transverse momentum $\mathbf{p}_{\mathbf{N}, \perp}$ and invariant mass $\tilde{M}_{N}^{2}$. The normalization condition for such nuclear spectral function is defined from the requirements of the baryonic number and the total light-front momentum conservation laws $[41,43]$ :

$$
\begin{aligned}
& \sum_{N=1}^{A} \int P_{A}^{N}\left(\alpha_{N}, \mathrm{p}_{N, \perp}, \tilde{M}_{N}^{2}\right) \frac{d \alpha_{N}}{2 \alpha_{N}} d^{2} \mathbf{p}_{N, \perp} d \tilde{M}_{N}^{2}=A, \\
& \sum_{N=1}^{A} \int \alpha_{N} P_{A}^{N}\left(\alpha_{N}, \mathrm{p}_{N, \perp}, \tilde{M}_{N}^{2}\right) \frac{d \alpha_{N}}{2 \alpha_{N}} d^{2} \mathbf{p}_{N, \perp} d \tilde{M}_{N}^{2}=A,
\end{aligned}
$$

where the second relation is exact if it is assumed that all the momentum in the nucleus is carried by the constituent nucleons.

The LF density matrix $\rho_{A}^{N}\left(\alpha_{N}, \mathrm{p}_{N, \perp}\right)$ is defined as the joint probability of finding a bound nucleon in the nucleus with light-front momentum fraction $\alpha_{N}$ and transverse momentum $\mathbf{p}_{\mathbf{N}, \perp}$. Hence, the following relation between the LF nuclear spectral function and the LF density matrix is obtained

$$
\rho_{A}^{N}\left(\alpha_{N}, \mathrm{p}_{N, \perp}\right)=\int P_{A}^{N}\left(\alpha_{N}, \mathrm{p}_{N, \perp}, \tilde{M}_{N}^{2}\right) \frac{1}{2} d \tilde{M}_{N}^{2}
$$


Then, from Eqs. (2.53) and (2.54) it follows that the normalization of the LF density matrix is given by:

$$
\begin{aligned}
& \sum_{N=1}^{A} \int \rho_{A}^{N}\left(\alpha_{N}, \mathrm{p}_{N, \perp}\right) \frac{d \alpha_{N}}{\alpha_{N}} d^{2} \mathbf{p}_{N, \perp}=A \\
& \sum_{N=1}^{A} \int \alpha_{N} \rho_{A}^{N}\left(\alpha_{N}, \mathrm{p}_{N, \perp}\right) \frac{d \alpha_{N}}{\alpha_{N}} d^{2} \mathbf{p}_{N, \perp}=A
\end{aligned}
$$

To obtain the models of the LF nuclear spectral functions, similar to the VN approximation case, the mean field, the $2 \mathrm{~N}$, and the $3 \mathrm{~N}$ SRC contributions are separately considered by following the decomposition of Fig. 2.2. In the LF approximation, the cut diagrams of Fig. 2.2 and 2.3 will be evaluated at the positive light-front ("-" component) energy poles of the spectator residual system. For the mean field contribution, it corresponds to the positive lightfront energy pole of the coherent $A-1$ residual nuclear system. For the 2N SRC contribution, they correspond to the positive light-front energy poles of the correlated nucleon and the $A-2$ residual nuclear system, whereas for the 3N SRC contribution, they correspond to the positive light-front energy poles of the two correlated nucleons.

\subsubsection{Nuclear spectral function in light-front approximation for a nucleon in the nuclear mean field}

To calculate the light-front nuclear spectral function for a nucleon in the nuclear mean field a procedure similar to the $\mathrm{VN}$ approximation case may be applied. Thus, after defining the effective vertex $\hat{V}_{M F}$ in the mean field covariant amplitude Eq.(2.9), the integral of the denominator of the propagator of the on-shell $(A-1)$ residual nuclear system will be calculated. With the result of the integration plus the definition of the numerator of the propagator of the onshell $(A-1)$ system, similar to Eq. (2.18), the mean field LF nuclear spectral function will be expressed through the unknown light-front mean field wave function of the nucleus.

A different approach is used in the present dissertation that is developed because of the fact that the mean field nuclear spectral function is dominating at small momenta and small removal energies $\left(<k_{F}\right)$ of the bound nucleon, for which the application of the nonrelativistic 
limit of the light-front approximation is well justified. Then, what is only needed is a relation between the mean field LF spectral function $P_{A, M F}^{N}\left(\alpha_{1}, \mathrm{p}_{1, \perp}, \tilde{M}_{N}^{2}\right)$ and the VN approximation mean field spectral function, $S_{A, M F}^{N}\left(\mathrm{p}, E_{m}\right)$ [Eq.2.21], in the nonrelativistic limit ${ }^{1}$. The relation between $P_{A, M F}^{N}$ and $S_{A, M F}^{N}$ can be found by using the normalization condition:

$$
\int P_{A, M F}^{N}\left(\alpha_{1}, \mathrm{p}_{1, \perp}, \tilde{M}_{N}^{2}\right) \frac{d \alpha_{1}}{2 \alpha_{1}} d^{2} \mathbf{p}_{1, \perp} d \tilde{M}_{N}^{2}=\int S_{A, M F}^{N}\left(\mathrm{p}_{1}, E_{m}\right) d E_{m} d^{3} \mathbf{p}_{1}=n_{M F}^{N},
$$

where $n_{M F}^{N}$ is the contribution to the total normalization of the nuclear spectral function. The Jacobian for the change of variables from the LF phase space [Eq.2.52] to the VN phase space, $d E_{m} d^{3} \mathbf{p}_{1}$, is calculated by considering that the total energy of the on-shell $A-1$ residual nuclear system is given by

$$
E_{A-1}=\sqrt{M_{A-1}^{2}+p_{A-1}^{2}}=M_{A}-M_{N}+E_{m}+\frac{\mathrm{p}_{1}^{2}}{2 M_{A-1}^{0}},
$$

where the last part of the equation follows from the definition of the removal energy $E_{m}$ [Eq.(2.12)] which is inherently nonrelativistic. In the above expression $M_{A-1}$ is the mass of the $A-1$ system, which can be in the excited state, while $M_{A-1}^{0}$ represents the ground state mass of the residual nucleus. The above equation results in the following expressions for $\alpha_{1}$

$$
\alpha_{1}=\frac{A p_{1}^{+}}{p_{A}^{+}}=\frac{A\left(p_{A}^{+}-p_{A-1}^{+}\right)}{p_{A}^{+}}=A-\frac{E_{A-1}+\mathrm{p}_{A-1, z}}{p_{A}^{+}}=A-\frac{E_{A-1}-\mathrm{p}_{1, z}}{p_{A}^{+}}
$$

and for $\tilde{M}_{N}^{2}$ :

$$
\begin{aligned}
\tilde{M}_{N}^{2} & =p_{1}^{-} p_{1}^{+}-\mathbf{p}_{1, \perp}^{2}=p_{1}^{+}\left(p_{A}^{-}-p_{A-1}^{-}\right)-\mathbf{p}_{1, \perp}^{2}=p_{1}^{+}\left(\frac{M_{A}^{2}}{p_{A}^{+}}-\frac{M_{A-1}^{2}+\mathbf{p}_{1, \perp}^{2}}{p_{A-1}^{-}}\right)-\mathbf{p}_{1, \perp}^{2} \\
& =\frac{p_{1}^{+}}{p_{A}^{+}}\left(M_{A}^{2}-\frac{M_{A-1}^{2}+\mathbf{p}_{1, \perp}^{2}}{p_{A-1}^{-} / p_{A}^{+}}\right)-\mathbf{p}_{1, \perp}^{2}=\frac{\alpha_{1}}{A}\left(M_{A}^{2}-\frac{M_{A-1}^{2}+\mathbf{p}_{1, \perp}^{2}}{\left(A-\alpha_{1}\right) / A}\right)-\mathbf{p}_{1, \perp}^{2}
\end{aligned}
$$

where $\mathbf{p}_{A, \perp}=0$, and the relation $\alpha_{A-1}=A-\alpha_{1}$ has been applied.

\footnotetext{
${ }^{1}$ Note that hereafter $\alpha_{N}$ and $p_{N, \perp}$ will be identified with $\alpha_{1}$ and $p_{1, \perp}$ respectively, giving the subscript "1" to the bound nucleon.
} 
Using Eqs. (2.58) and (2.59) yields

$$
\begin{aligned}
\frac{\partial \alpha_{1}}{\partial \mathrm{p}_{1, \mathrm{z}}} & =\frac{A}{p_{A}^{+}}\left(1-\frac{\partial E_{A-1}}{\partial \mathrm{p}_{1, \mathrm{z}}}\right)=\frac{A}{\mathrm{p}_{\mathrm{A}}^{+}}\left(\frac{M_{A-1}^{0}-p_{1, z}}{M_{A-1}^{0}}\right) \\
\frac{\partial \alpha_{1}}{\partial E_{m}} & =\frac{\partial \alpha_{1}}{\partial E_{A-1}} \frac{\partial E_{A-1}}{\partial E_{m}}=-\frac{A}{\mathrm{p}_{\mathrm{A}}^{+}}, \\
\frac{\partial \tilde{M}_{N}^{2}}{\partial \mathrm{p}_{1, \mathrm{z}}} & =\frac{\partial \tilde{M}_{N}^{2}}{\partial \alpha_{1}} \frac{\partial \alpha_{1}}{\partial \mathrm{p}_{1, \mathrm{z}}}+\frac{\partial \tilde{M}_{N}^{2}}{\partial M_{A-1}^{2}} \frac{\partial M_{A-1}^{2}}{\partial \mathrm{p}_{1, \mathrm{z}}} \\
& =\left(\frac{M_{A-1}^{0}-\mathrm{p}_{1, \mathrm{z}}}{M_{A-1}^{0}}\right)\left[\frac{M_{A}^{2}}{\mathrm{p}_{\mathrm{A}}^{+}}-\frac{A^{2}}{\mathrm{p}_{\mathrm{A}}^{+}} \frac{M_{A-1}^{2}+\mathrm{p}_{1, \perp}^{2}}{\left(A-\alpha_{1}\right)^{2}}\right]-\left(\frac{2 \alpha_{1}}{A-\alpha_{1}}\right)\left(\frac{E_{A-1}-M_{A-1}^{0}}{M_{A-1}^{0}}\right) \mathrm{p}_{1, \mathrm{z}}, \\
\frac{\partial \tilde{M}_{N}^{2}}{\partial E_{m}} & =\frac{\partial \tilde{M}_{N}^{2}}{\partial \alpha_{1}} \frac{\partial \alpha_{1}}{\partial E_{m}}+\frac{\partial \tilde{M}_{N}^{2}}{\partial M_{A-1}^{2}} \frac{\partial M_{A-1}^{2}}{\partial E_{m}}=-\frac{M_{A}^{2}}{\mathrm{p}_{\mathrm{A}}^{+}}+\frac{A^{2}}{\mathrm{p}_{\mathrm{A}}^{+}} \frac{M_{A-1}^{2}+\mathrm{p}_{1, \perp}^{2}}{\left(A-\alpha_{1}\right)^{2}}-\left(\frac{2 \alpha_{1}}{A-\alpha_{1}}\right) E_{A-1},
\end{aligned}
$$

thus the Jacobian for the change of variables $\left(\alpha_{1}, \tilde{M}_{N}^{2}\right)$ to $\left(E_{m}, \mathrm{p}_{1, \mathrm{z}}\right)$ is given by

$$
J=\frac{\partial \alpha_{1}}{\partial \mathrm{p}_{1, \mathrm{z}}} \frac{\partial \tilde{M}_{N}^{2}}{\partial E_{m}}-\frac{\partial \alpha_{1}}{\partial E_{m}} \frac{\partial \tilde{M}_{N}^{2}}{\partial \mathrm{p}_{1, \mathrm{z}}}=-\frac{2 \alpha_{1}}{\alpha_{A-1}}\left(\frac{A \mathrm{p}_{\mathrm{A}-1}^{+}}{\mathrm{p}_{\mathrm{A}}^{+}}\right)=-2 \alpha_{1} .
$$

Then

$$
d \tilde{M}_{N}^{2} d \alpha_{1}=|J| d E_{m} d p_{1, z}=2 \alpha_{1} d E_{m} d p_{1, z}
$$

The above equation, together with Eq. (2.52), results in

$$
d^{4} p_{1}=\frac{d \alpha_{1}}{2 \alpha_{1}} d^{2} \mathbf{p}_{1, \perp} d \tilde{M}_{N}^{2}=d E_{m} d^{3} \mathbf{p}_{1}
$$

which inserted in Eq. (2.56) results in the following relation between the mean field nuclear spectral functions $P_{A, M F}^{N}\left(\alpha_{1}, \mathrm{p}_{1, \perp}, \tilde{M}_{N}^{2}\right)$ and $S_{A, M F}^{N}\left(\mathrm{p}_{1}, E_{m}\right)$

$$
P_{A, M F}^{N}\left(\alpha_{1}, \mathrm{p}_{1, \perp}, \tilde{M}_{N}^{2}\right)=S_{A, M F}^{N}\left(\mathrm{p}_{1}, E_{m}\right)
$$

where $\alpha_{1}$ and $\tilde{M}_{N}^{2}$ are expressed through $E_{m}$ and $\mathrm{p}_{1}$ according to Eqs. (2.57)-(2.59). Note that the above equation is valid for up to the overall normalization factor, since VN and LF approximations result in different normalizations for the mean field contribution to the nuclear spectral function. 


\subsubsection{Nuclear spectral function in light-front approximation for two nucleons in short-range correlation}

The calculation of the nuclear spectral function in light-front approximation for two nucleons in short-range correlation starts with the definition of the effective vertex $\hat{V}_{2 N}$ in the 2N SRC covariant amplitude (2.10) [125]:

$$
\hat{V}_{2 N}=i \bar{a}\left(p_{1}, s_{1}\right) 2 \alpha_{1}^{2} \delta\left(\alpha_{1}+\alpha_{2}+\alpha_{A-2}-A\right) \delta^{2}\left(\mathbf{p}_{1, \perp}+\mathbf{p}_{2, \perp}+\mathbf{p}_{A-2, \perp}\right) \delta\left(\tilde{M}_{N}^{2}-\tilde{M}_{N}^{(2 N), 2}\right) a\left(p_{1}, s_{1}\right)
$$

where $\left(\alpha_{2}, \mathbf{p}_{2, \perp}\right),\left(\alpha_{A-2}, \mathbf{p}_{A-2, \perp}\right)$ are the light-front momentum fractions and the transverse momenta of the correlated second nucleon and of the $A-2$ residual nuclear system respectively, and $\mathbf{p}_{A, \perp}=0$. The creation, $\bar{a}\left(p_{1}, s_{1}\right)$, and the annihilation, $a\left(p_{1}, s_{1}\right)$, operators of a nucleon with four-momentum $p_{1}$ and spin $s_{1}$ satisfy the relations of Eq. (2.16). The delta functions represent the momentum and the invariant mass conservation in the vertex.

Since in the light-front NN correlation model, the particle 2 and the $A-2$ residual nuclear

system are considered on light-front energy shells, the magnitude of the invariant mass $\tilde{M}_{N}^{(2 N), 2}$ is calculated as follows

$$
\begin{aligned}
\tilde{M}_{N}^{(2 N), 2}= & p_{1}^{-} p_{1}^{+}-\mathrm{p}_{1, \perp}^{2}=p_{1}^{+}\left(p_{A}^{-}-p_{2}^{-}-p_{A-2}^{-}\right)-\mathrm{p}_{1, \perp}^{2} \\
& =p_{1}^{+}\left(\frac{M_{A}^{2}}{p_{A}^{+}}-\frac{M_{N}^{2}+\mathrm{p}_{2, \perp}^{2}}{p_{2}^{+}}-\frac{M_{A-2}^{2}+\mathrm{p}_{A-2, \perp}^{2}}{p_{A-2}^{+}}\right)-\mathrm{p}_{1, \perp}^{2} \\
& =\frac{p_{1}^{+}}{p_{A}^{+}}\left(M_{A}^{2}-\frac{M_{N}^{2}+\mathrm{p}_{2 \perp}^{2}}{p_{2}^{+} / p_{A}^{+}}-\frac{M_{A-2}^{2}+\mathrm{p}_{A-2, \perp}^{2}}{p_{A-2}^{+} / p_{A}^{+}}\right)-\mathrm{p}_{1 \perp}^{2} \\
& =\frac{\alpha_{1}}{A}\left(M_{A}^{2}-A \frac{M_{N}^{2}+\mathrm{p}_{2, \perp}^{2}}{\alpha_{2}}-A \frac{M_{A-2}^{2}+\mathrm{p}_{A-2, \perp}^{2}}{\alpha_{A-2}}\right)-\mathrm{p}_{1, \perp}^{2} \\
& =\frac{\alpha_{1}}{A}\left[M_{A}^{2}-A \frac{M_{N}^{2}+\left(\mathbf{p}_{A-2, \perp}-\mathbf{p}_{1, \perp}\right)^{2}}{A-\alpha_{1}-\alpha_{A-2}}-A \frac{M_{A-2}^{2}+\mathrm{p}_{A-2, \perp}^{2}}{\alpha_{A-2}}\right]-\mathrm{p}_{1, \perp}^{2},
\end{aligned}
$$

where $\mathbf{p}_{A-2, \perp}=\mathbf{p}_{1, \perp}+\mathbf{p}_{2, \perp}$, and the $z$ axis in the reference frame is taken in the $\mathbf{p}_{A}$ direction.

Similar to the propagator integration described in section 2.3.1, the integrations by $d p_{2}^{-}$ and $d p_{A-2}^{-}$in Eq. (2.10) are performed through the " - " components of the positive poles 
of the propagators of the on-shell particle 2 and the $A-2$ residual nuclear system respectively, provided that their " + " components are large and positive, so that the $Z$ diagram in this scheme will be suppressed by the inverse power of large " + " component of the nucleon's four-momentum [25]. The integration by $d p_{2}^{-}$of the propagator of particle 2 yields

$$
\begin{aligned}
\oint \frac{d^{4} p_{2}}{p_{2}^{2}-M_{N}+i \varepsilon} & =\oint \frac{d p_{2}^{-} d p_{2}^{+} d^{2} \mathrm{p}_{2, \perp}}{2\left(p_{2}^{+} p_{2}^{-}-\mathrm{p}_{2, \perp}^{2}-M_{N}\right)+i \varepsilon} \\
& =\oint \frac{d p_{2}^{-} d p_{2}^{+} d^{2} \mathrm{p}_{2, \perp}}{2 p_{2}^{+}\left[p_{2}^{-}-\left(\mathrm{p}_{2, \perp}^{2}+M_{N}\right) / p_{2}^{+}\right]+i \varepsilon}=-\left.\frac{2 \pi i d p_{2}^{+} d^{2} \mathbf{p}_{2, \perp}}{2 p_{2}^{+}}\right|_{p_{2}^{-}=\frac{\mathrm{p}_{2 \perp}^{2}+M_{N}}{p_{2}^{+}}} \\
& =-\left.\pi i \frac{d \alpha_{2}}{\alpha_{2}} d^{2} \mathbf{p}_{2, \perp}\right|_{p_{2}^{-}=\frac{\mathrm{p}_{2, \perp}^{2}+M_{N}}{p_{2}^{+}}} .
\end{aligned}
$$

Similarly, integration by $d p_{A-2}^{-}$in Eq.(2.10) over the positive pole of the propagator of the $A-2$ residual nuclear system yields

$$
\begin{aligned}
\oint \frac{d^{4} p_{A-2}}{p_{A-2}^{2}-M_{A-2}+i \varepsilon} & =\oint \frac{\mathrm{d}^{2} \mathrm{p}_{A-2}^{-} \mathrm{dp}_{A-2}^{+} \mathrm{d}_{A-2, \perp}}{2\left(\mathrm{p}_{A-2}^{+} \mathrm{p}_{A-2}^{-}-\mathrm{p}_{A-2, \perp}^{2}-M_{A-2}\right)+i \varepsilon} \\
& =-\left.\pi i \frac{d \alpha_{A-2}}{\alpha_{A-2}} d^{2} \mathbf{p}_{A-2, \perp}\right|_{\mathrm{p}_{A-2}^{-}}=\frac{\mathrm{p}_{A-2, \perp}^{2}+M_{A-2}}{p_{A-2}^{+}}
\end{aligned}
$$

The above integrations project the intermediate state to the positive light-front energy state thus excluding the contribution from the Z graph of Fig. 2.1(c). With the diminished contribution from the $Z$ graph, the 2N SRC covariant amplitude [Eq. (2.10) ] will result in the 2N SRC light-front spectral function, $P_{A, 2 N}^{N}\left(\alpha_{1}, p_{1, \perp}, \tilde{M}_{N}^{2}\right)$.

As in the VN approximation, if $\chi_{A-2}$ is the LF spin wave function of the on-shell $A-2$ residual nuclear system, then the numerator of its propagator in Eq. (2.10), can be expressed by the following sum rule representing the completeness relation for the wave function:

$$
G\left(p_{A-2}, s_{A-2}\right)=\sum_{s_{A-2}} \chi_{A-2}\left(p_{A-2}, s_{A-2}\right) \chi_{A-2}^{\dagger}\left(p_{A-2}, s_{A-2}\right)
$$


where $s_{A-2}$ is the spin projection of the $A-2$ residual nuclear system. Similarly, the numerator of the propagator for the on-shell particle 2 is given by

$$
\not p_{2}+M_{N}=\sum_{s 2} u\left(p_{2}, s_{2}\right) \bar{u}\left(p_{2}, s_{2}\right)
$$

where $u\left(p_{2}, s_{2}\right)$ is the spinor of particle 2 with momentum $p_{2}$ and spin projection $s_{2}$ [40].

In the nonrelativistic limit for the center of mass motion of the $2 \mathrm{~N} \mathrm{SRC,} k_{C M} \ll M_{N N}$ [for $k_{C M}$ see Eq. (2.89) below], the propagator $G\left(p_{N N}, s_{N N}\right)$ can be approximated as

$$
G\left(p_{N N}, s_{N N}\right) \approx \sum_{s_{N N}} \chi_{N N}\left(p_{N N}, s_{N N}\right) \chi_{N N}^{\dagger}\left(p_{N N}, s_{N N}\right)
$$

where $\chi_{N N}$ is the spin wave function of the center of mass motion of the $2 \mathrm{~N}$ SRC.

Inserting Eqs. (2.16) and (2.67) -(2.71) in the covariant 2N SRC amplitude [Eq. (2.10)], and summing over all possible spin projections $s_{2}, s_{A-2}$ and $s_{N N}$, reduces the latter to the $2 \mathrm{~N}$ SRC part of the nuclear spectral function in LF approximation, namely:

$$
\begin{aligned}
P_{A, 2 N}^{N}\left(\alpha_{1}, \mathrm{p}_{1, \perp}, \tilde{M}_{N}^{2}\right) & =\sum_{s_{2}, s_{N N}, s_{A-2}} \int \chi_{A}^{s_{A}, \dagger} \Gamma_{A \rightarrow N N, A-2}^{\dagger} \chi_{A-2}\left(p_{A-2}, s_{A-2}\right) \frac{\chi_{N N}\left(p_{N N}, s_{N N}\right)}{p_{N N}^{2}-M_{N N}^{2}} \\
& \times \chi_{N N}^{\dagger}\left(p_{N N}, s_{N N}\right) \Gamma_{N N \rightarrow N N}^{\dagger} \frac{u\left(p_{1}, s_{1}\right) u\left(p_{2}, s_{2}\right)}{p_{1}^{2}-M_{N}^{2}} \\
& \times 2 \alpha_{1}^{2} \delta\left(\alpha_{1}+\alpha_{2}+\alpha_{A-2}-A\right) \delta^{2}\left(\mathbf{p}_{1, \perp}+\mathbf{p}_{2, \perp}+\mathbf{p}_{A-2, \perp}\right) \delta\left(\tilde{M}_{N}^{2}-\tilde{M}_{N}^{(2 N), 2}\right) \\
& \times \frac{\bar{u}\left(p_{1}, s_{1}\right) \bar{u}\left(p_{2}, s_{2}\right)}{p_{1}^{2}-M_{N}^{2}} \Gamma_{N N \rightarrow N, N} \chi_{N N}\left(p_{N N}, s_{N N}\right) \\
& \times \frac{\chi_{N N}^{\dagger}\left(p_{N N}, s_{N N}\right)}{p_{N N}^{2}-M_{N N}^{2}} \chi_{A-2}^{\dagger}\left(p_{A-2}, s_{A-2}\right) \Gamma_{A \rightarrow N N, A-2} \chi_{A}^{s_{A}} \\
& \times \frac{d \alpha_{2}}{\alpha_{2}} \frac{d^{2} \mathbf{p}_{2, \perp}}{2(2 \pi)^{3}} \frac{d \alpha_{A-2}}{\alpha_{A-2}} \frac{d^{2} \mathbf{p}_{A-2, \perp}}{2(2 \pi)^{3}}
\end{aligned}
$$




\section{Light-front wave function of the NN SRC}

The calculation of the light-front wave function of the NN SRC is based on the consideration of the following expression, as well as its complex conjugated form, in Eq. (2.72):

$$
\frac{\bar{u}\left(p_{1}, s_{1}\right) \bar{u}\left(p_{2}, s_{2}\right)}{p_{1}^{2}-M_{N}^{2}} \Gamma_{N N \rightarrow N N} \chi_{N N}\left(p_{N N}, s_{N N}\right) .
$$

On the basis of the light-front momentum and energy conservation at the $\Gamma_{N N \rightarrow N, N}$ vertex, and on the on-shell condition of particle 2, the propagator in Eq. (2.73) can be expressed as

$$
\begin{aligned}
p_{1}^{2}-M_{N}^{2} & =\left(p_{N N}-p_{2}\right)^{2}-M_{N}^{2}=\left(p_{N N}^{+}-p_{2}^{+}\right)\left(p_{N N}^{-}-p_{2}^{-}\right)-\left(\mathbf{p}_{N N, \perp}-\mathbf{p}_{2, \perp}\right)^{2}-M_{N}^{2} \\
& =\left(p_{N N}^{+}-p_{2}^{+}\right)\left(p_{N N}^{-}-p_{2}^{-}-\frac{M_{N}^{2}+\mathrm{p}_{1 \perp}^{2}}{p_{N N}^{+}-p_{2}^{+}}\right) \\
& =p_{1}^{+}\left(\frac{M_{N N}^{2}+\mathrm{p}_{N N, \perp}^{2}}{p_{N N}^{+}}-\frac{M_{N}^{2}+\mathrm{p}_{2, \perp}^{2}}{p_{2}^{+}}-\frac{M_{N}^{2}+\mathrm{p}_{1, \perp}^{2}}{p_{1}^{+}}\right) \\
& =\frac{\alpha_{1}}{\alpha_{N N}}\left\{M_{N N}^{2}+\mathrm{p}_{N N, \perp}^{2}-\frac{\alpha_{N N}}{\alpha_{1} \alpha_{2}}\left[\left(M_{N}^{2}+\mathrm{p}_{2, \perp}^{2}\right) \alpha_{1}-\left(M_{N}^{2}+\mathrm{p}_{1, \perp}^{2}\right) \alpha_{2}\right]\right\},
\end{aligned}
$$

where it has been assumed that $k_{C M} \ll M_{N N}$, which justifies the approximation, $p_{N N}^{-} \approx \frac{M_{N N}^{2}+\mathrm{p}_{N N, 1}^{2}}{p_{N N}^{+}}$. Eq. (2.74) can be further simplified by using the relations $\alpha_{1}+\alpha_{2}=\alpha_{N N}$ and $\mathbf{p}_{1, \perp}+\mathbf{p}_{2, \perp}=\mathbf{p}_{N N, \perp}$, namely

$$
\begin{aligned}
p_{1}^{2}-M_{N}^{2} & =\frac{\alpha_{1}}{\alpha_{N N}}\left[M_{N N}^{2}-\frac{\alpha_{N N}^{2}}{\alpha_{1} \alpha_{2}}\left(M_{N}^{2}+\mathrm{p}_{1, \perp}^{2}+\frac{\alpha_{1}^{2}}{\alpha_{N N}^{2}} \mathrm{p}_{N N, \perp}^{2}-\frac{2 \alpha_{1}}{\alpha_{N N}} \mathbf{p}_{1, \perp} \mathbf{p}_{N N, \perp}\right)\right] \\
& =\frac{\alpha_{1}}{\alpha_{N N}}\left\{M_{N N}^{2}-\frac{\alpha_{N N}^{2}}{\alpha_{1} \alpha_{2}}\left[M_{N}^{2}+\left(\mathbf{p}_{1 \perp}-\frac{\alpha_{1}}{\alpha_{N N}} \mathbf{p}_{N N, \perp}\right)^{2}\right]\right\} . \\
& =\frac{\alpha_{1}}{\alpha_{N N}}\left(M_{N N}^{2}-s_{N N}\right),
\end{aligned}
$$

where the invariant squared energy of the $N N$ system, $s_{N N}$, is given by

$$
\begin{aligned}
s_{N N} & =p_{N N}^{2}=\left(p_{1}+p_{2}\right)^{2}=\left(p_{1}^{+}+p_{2}^{+}\right)\left(p_{1}^{-}+p_{2}^{-}\right)-\left(\mathbf{p}_{1, \perp}+\mathbf{p}_{2, \perp}^{+}\right)^{2} \\
& =\frac{\alpha_{N N}^{2}}{\alpha_{1} \alpha_{2}}\left[M_{N}^{2}+\left(\mathbf{p}_{1 \perp}-\frac{\alpha_{1}}{\alpha_{N N}} \mathbf{p}_{N N, \perp}\right)^{2}\right] .
\end{aligned}
$$


The propagator [Eq.(2.75)] can be completely expressed though the internal momenta of the $N N$ system by introducing the momentum fraction of the 2N SRC carried by the nucleon $1, \beta_{1}$, and the relative transverse momentum, $\mathbf{k}_{1, \perp}$, as follows:

$$
\begin{array}{r}
\beta_{1}=2-\beta_{2}=\frac{2 \alpha_{1}}{\alpha_{N N}}, \\
\mathbf{k}_{1, \perp}=\mathbf{p}_{1, \perp}-\frac{\beta_{1}}{2} \mathbf{p}_{N N, \perp} .
\end{array}
$$

The propagator [Eq.(2.75)] can therefore be expressed as

$$
p_{1}^{2}-M_{N}^{2}=\frac{\beta_{1}}{2}\left[M_{N N}^{2}-\frac{4}{\beta_{1}\left(2-\beta_{1}\right)}\left(M_{N}^{2}+\mathrm{k}_{1, \perp}^{2}\right)\right] \text {, }
$$

and the invariant squared energy $s_{N N}$ as

$$
s_{N N}=\frac{4}{\beta_{1}\left(2-\beta_{1}\right)}\left(M_{N}^{2}+\mathrm{k}_{1, \perp}^{2}\right)=4\left(M_{N}^{2}+\mathrm{k}_{1}^{2}\right),
$$

where the relative momentum, $\mathbf{k}_{1}$, in the $N N$ center of mass frame, is invariant with respect to the Lorentz boost in the $\mathbf{p}_{\mathrm{NN}}$ direction. The relative momentum $\mathbf{k}_{1}$ will be used to set a momentum scale for the $2 \mathrm{~N}$ SRCs, requiring $\mathrm{k}_{1} \geq \mathrm{k}_{s r c}$ similar to Eq. (2.37).

Inserting Eqs. (2.79) and (2.80) in Eq. (2.73) yields

$$
\begin{gathered}
\frac{\bar{u}\left(p_{1}, s_{1}\right) \bar{u}\left(p_{2}, s_{2}\right)}{\frac{\beta_{1}}{2}\left[M_{N N}^{2}-\frac{4}{\beta_{1}\left(2-\beta_{1}\right)}\left(M_{N}^{2}+\mathrm{k}_{1}^{2}\right)\right]} \Gamma_{N N \rightarrow N N} \chi_{N N}\left(p_{N N}, s_{N N}\right) \\
=\frac{\bar{u}\left(p_{1}, s_{1}\right) \bar{u}\left(p_{2}, s_{2}\right)}{\frac{\beta_{1}}{2}\left[M_{N N}^{2}-4\left(M_{N}^{2}+\mathrm{k}_{1}^{2}\right)\right]} \Gamma_{N N \rightarrow N N} \chi_{N N}\left(p_{N N}, s_{N N}\right) .
\end{gathered}
$$

The expression in Eq. (2.81) can be presented in a more compact form by introducing the light-front wave function of the $N N$ SRC $[36,125]$ in the form:

$$
\psi_{N N}^{s_{N N}}\left(\beta_{1}, k_{1, \perp}, s_{1}, s_{2}\right)=-\frac{1}{\sqrt{2(2 \pi)^{3}}} \frac{\bar{u}\left(p_{1}, s_{1}\right) \bar{u}\left(p_{2}, s_{2}\right) \Gamma_{N N \rightarrow N N} \chi_{N N}\left(p_{N N}, s_{N N}\right)}{\frac{1}{2}\left[M_{N N}^{2}-4\left(M_{N}^{2}+\mathrm{k}_{1}^{2}\right)\right]}
$$


The above defined wave function results in the following expression for Eq. (2.73)

$$
\frac{\bar{u}\left(p_{1}, s_{1}\right) \bar{u}\left(p_{2}, s_{2}\right)}{p_{1}^{2}-M_{N}^{2}} \Gamma_{N N \rightarrow N N} \chi_{N N}\left(p_{N N}, s_{N N}\right)=-\frac{\sqrt{2(2 \pi)^{3}}}{\beta_{1}} \psi_{N N}^{s_{N N}}\left(\beta_{1}, \mathrm{k}_{1, \perp}, s_{1}, s_{2}\right) .
$$

\section{Light-front wave function of the NN - (A-2) System}

The light-front wave function of the $N N-(A-2)$ system describes the motion of the center of mass of the NN correlation. The calculation of this wave function depends on the consideration of the following expression, as well as its complex conjugated form, in Eq. (2.72):

$$
\frac{\chi_{N N}^{\dagger}\left(p_{N N}, s_{N N}\right) \chi_{A-2}^{\dagger}\left(p_{A-2}, s_{A-2}\right)}{p_{N N}^{2}-M_{N N}^{2}} \Gamma_{A \rightarrow N N, A-2} \chi_{A} .
$$

On the basis of the light-front momentum and energy conservation at the $\Gamma_{A \rightarrow N N, A-2}$ vertex, and on the on-shell conditions of the initial nucleus $A$ and the $A-2$ residual nuclear system, the propagator in Eq. (2.84) can be expressed as

$$
\begin{aligned}
p_{N N}^{2}-M_{N N}^{2} & =\left(p_{A}-p_{A-2}\right)^{2}-M_{N N}^{2}=\left(p_{A}^{+}-p_{A-2}^{+}\right)\left(p_{A}^{-}-p_{A-2}^{-}\right)-\left(\mathbf{p}_{A, \perp}-\mathbf{p}_{A-2, \perp}\right)^{2}-M_{N N}^{2} \\
& =\left(p_{A}^{+}-p_{A-2}^{+}\right)\left(p_{A}^{-}-p_{A-2}^{-}-\frac{\left(\mathbf{p}_{A, \perp}-\mathbf{p}_{A-2, \perp}\right)^{2}}{p_{A}^{+}-p_{A-2}^{+}}-\frac{M_{N N}^{2}}{p_{A}^{+}-p_{A-2}^{+}}\right) \\
& =p_{N N}^{+}\left(\frac{M_{A}^{2}+\mathrm{p}_{A, \perp}^{2}}{p_{A}^{+}}-\frac{M_{A-2}^{2}+\mathrm{p}_{A-2, \perp}^{2}}{p_{A-2}^{+}}-\frac{\mathrm{p}_{A, \perp}^{2}-2 \mathbf{p}_{A, \perp} \mathbf{p}_{A-2, \perp}+\mathrm{p}_{A-2, \perp}^{2}}{p_{N N}^{+}}-\frac{M_{N N}^{2}}{p_{N N}^{+}}\right) \\
& =\frac{\alpha_{N N}}{A}\left\{M_{A}^{2}-\frac{A^{2}}{\alpha_{N N}\left(A-\alpha_{N N}\right)}\left[M_{N N}^{2}+\frac{\alpha_{N N}}{A}\left(M_{A-2}^{2}-M_{N N}^{2}\right)+\left(p_{N N, \perp}-\frac{\alpha_{N N}}{A} p_{A, \perp}\right)^{2}\right]\right\} \\
& =\frac{\alpha_{N N}}{A}\left(M_{A}^{2}-s_{N N, A-2}\right),
\end{aligned}
$$

where the invariant squared energy $s_{N N, A-2}$, of the $N N-(A-2)$ system is given by

$$
\begin{aligned}
s_{N N, A-2} & =\left(p_{N N}+p_{A-2}\right)^{2}=\left(p_{N N}^{+}+p_{A-2}^{+}\right)\left(p_{N N}^{-}+p_{A-2}^{-}\right)-\left(\mathbf{p}_{N N, \perp}+\mathbf{p}_{A-2, \perp}\right)^{2} \\
& =\frac{A^{2}}{\alpha_{N N}\left(A-\alpha_{N N}\right)}\left[M_{N N}^{2}+\frac{\alpha_{N N}}{A}\left(M_{A-2}^{2}-M_{N N}^{2}\right)+\left(\mathbf{p}_{N N, \perp}-\frac{\alpha_{N N}}{A} \mathbf{p}_{A, \perp}\right)^{2}\right] .
\end{aligned}
$$


The relative three-momentum, $\mathbf{k}_{C M}$, in the center of mass of the $N N-(A-2)$ system is defined such that $\mathbf{k}_{C M}=\mathbf{p}_{\mathrm{NN}}=-\mathbf{p}_{\mathrm{A}-2}$, then

$$
\begin{aligned}
& E_{N N}=\sqrt{M_{N N}^{2}+\mathrm{k}_{C M}^{2}}, \\
& E_{A-2}=\sqrt{M_{A-2}^{2}+\mathrm{k}_{C M}^{2}},
\end{aligned}
$$

that implies that the invariant squared energy, $s_{N N, A-2}$ [Eq. (2.86)], is given by

$$
s_{N N, A-2}=\left(p_{N N}+p_{A-2}\right)^{2}=\left(E_{N N}+E_{A-2}\right)^{2}=M_{N N}^{2}+2 \mathrm{k}_{C M}^{2}+2 \sqrt{\left(M_{N N}^{2}+\mathrm{k}_{C M}^{2}\right)\left(M_{A-2}^{2}+\mathrm{k}_{C M}^{2}\right)},
$$

the magnitude of $\mathbf{k}_{C M}$ is therefore given by:

$$
\mathrm{k}_{C M}=\frac{\sqrt{\left[s_{N N, A-2}-\left(M_{N N}+M_{A-2}\right)^{2}\right]\left[s_{N N, A-2}-\left(M_{N N}-M_{A-2}\right)^{2}\right]}}{2 \sqrt{s_{N N, A-2}}},
$$

and the light-front momentum fraction of the $N N$ pair, in the center of mass of the $N N-(A-2)$ system, by

$$
\alpha_{N N}=A \frac{p_{N N}^{+}}{p_{A}^{+}}=\frac{A\left(E_{N N}+\mathrm{k}_{C M, z}\right)}{E_{N N}+E_{A-2}} .
$$

With the above definitions, similar to Eq. (2.82), the light-front wave function of the $N N-$ $(A-2)$ system is defined as:

$\psi_{C M}^{s_{A}}\left(\alpha_{N N}, \mathrm{k}_{N N, \perp}, s_{N N}, s_{A-2}\right)=-\frac{1}{\sqrt{\frac{A-2}{2}}} \frac{1}{\sqrt{2(2 \pi)^{3}}} \frac{\chi_{N N}^{\dagger}\left(p_{N N}, s_{N N}\right) \chi_{A-2}^{\dagger}\left(p_{A-2}, s_{A-2}\right) \Gamma_{A \rightarrow N N, A-2} \chi_{A}^{s_{A}}}{\frac{2}{A}\left[M_{A}^{2}-s_{N N, A-2}\right]}$,

where $\mathbf{k}_{N N, \perp}=\mathbf{p}_{N N, \perp}-\frac{\alpha_{N N}}{A} \mathbf{p}_{A, \perp}$. The coefficients in this wave function are chosen such that in the nonrelativistic limit, $k_{C M} \ll M_{N N}$ :

$$
\psi_{C M}^{s_{A}}\left(\alpha_{N N}, \mathrm{p}_{N N, \perp}, s_{N N}, s_{A-2}\right) \approx 2 M_{N} \psi_{C M}^{N R}\left(\mathrm{k}_{C M}\right),
$$


where $\psi_{C M}^{N R}\left(\mathrm{k}_{C M}\right)$ is the nonrelativistic wave function of the center of mass of the $N N-(A-2)$ system.

Substituting Eqs.(2.91) and (2.85) in Eq.(2.84) yields

$$
\frac{\chi_{N N}^{\dagger}\left(p_{N N}, s_{N N}\right) \chi_{A-2}^{\dagger}\left(p_{A-2}, s_{A-2}\right)}{p_{N N}^{2}-M_{N N}^{2}} \Gamma_{A \rightarrow N N, A-2} \chi_{A}=-\frac{\sqrt{\frac{A-2}{2}} \sqrt{2(2 \pi)^{3}}}{\alpha_{N N} / 2} \psi_{C M}^{s_{A}}\left(\alpha_{N N}, \mathrm{k}_{N N, \perp}, s_{N N}, s_{A-2}\right) .
$$

\section{N SRC light-front nuclear spectral function}

Inserting Eqs. (2.83) and (2.93) in Eq.(2.72), the following expression for the 2N SRC light-front nuclear spectral function is obtained

$$
\begin{aligned}
P_{A, 2 N}^{N}\left(\alpha_{1}, \mathrm{p}_{1, \perp}, \tilde{M}_{N}^{2}\right) & =\frac{A-2}{2} \sum_{s_{2}, s_{N N}, s_{A-2}} \int \psi_{C M}^{s_{A}, \dagger}\left(\alpha_{N N}, \mathrm{k}_{N N, \perp}, s_{N N}, s_{A-2}\right) \psi_{N N}^{s_{N N}, \dagger}\left(\beta_{1}, \mathrm{k}_{1, \perp}, s_{1}, s_{2}\right) \\
& \times 2 \delta\left(\alpha_{1}+\alpha_{2}+\alpha_{A-2}-A\right) \delta^{2}\left(\mathbf{p}_{1, \perp}+\mathbf{p}_{2, \perp}-\mathbf{p}_{N N, \perp}\right) \delta\left(\tilde{M}_{N}^{2}-\tilde{M}_{N}^{(2 N), 2}\right) \\
& \times \psi_{C M}^{s_{A}}\left(\alpha_{N N}, \mathrm{k}_{N N, \perp}, s_{N N}, s_{A-2}\right) \psi_{N N}^{s_{N N}}\left(\beta_{1}, \mathrm{k}_{1, \perp}, s_{1}, s_{2}\right) \\
& \times \frac{d \beta_{2}}{2-\beta_{1}} d^{2} \mathbf{p}_{2, \perp} \frac{d \alpha_{N N}}{A-\alpha_{N N}} d^{2} \mathbf{p}_{N N, \perp}
\end{aligned}
$$

where the relations $\beta_{2}=2-\beta_{1}, d \beta_{2} / \beta_{2}=d \alpha_{2} / \alpha_{2}, \mathbf{p}_{\mathbf{N N}, \perp}=-\mathbf{p}_{\mathbf{A}-\mathbf{2}, \perp}$, and $\alpha_{A-2}=A-\alpha_{N N}$ have been applied.

The nuclear spectral function [Eq.(2.94)] can be further simplified by introducing the spin averaged density matrices for the relative motion in the 2N SRC [36, 125]:

$$
\rho_{N N}^{N}\left(\beta_{1}, \mathrm{k}_{1, \perp}\right)=\frac{1}{2} \frac{1}{2 s_{N N}+1} \sum_{s_{N N}, s_{1}, s_{2}} \frac{\left|\psi_{N N}^{s_{N N}}\left(\beta_{1}, \mathrm{k}_{1, \perp}, s_{1}, s_{2}\right)\right|^{2}}{2-\beta_{1}}
$$

and for the center of mass motion of the 2N SRC:

$$
\rho_{C M}\left(\alpha_{N N}, \mathrm{k}_{N N, \perp}\right)=\frac{1}{2} \frac{A-2}{2 s_{A}+1} \sum_{s_{N N}, s_{A-2}} \frac{\left|\psi_{C M}^{s_{A}}\left(\alpha_{N N}, \mathrm{k}_{N N, \perp}, s_{N N}, s_{A-2}\right)\right|^{2}}{A-\alpha_{N N}} .
$$


With the above definitions, the 2N SRC light-front nuclear spectral function is given by

$$
\begin{array}{r}
P_{A, 2 N}^{N}\left(\alpha_{1}, \mathrm{p}_{1, \perp}, \tilde{M}_{N}^{2}\right)=\frac{1}{2} \int \rho_{N N}^{N}\left(\beta_{1}, \mathrm{k}_{1, \perp}\right) \rho_{C M}\left(\alpha_{N N}, \mathrm{k}_{N N, \perp}\right) 2 \delta\left(\alpha_{1}+\alpha_{2}-\alpha_{N N}\right) \\
\delta^{2}\left(\mathbf{p}_{1, \perp}+\mathbf{p}_{2, \perp}-\mathbf{p}_{N N, \perp}\right) \delta\left(\tilde{M}_{N}^{2}-\tilde{M}_{N}^{(2 N), 2}\right) d \beta_{2} d^{2} \mathbf{p}_{2, \perp} d \alpha_{N N} d^{2} \mathbf{p}_{N N, \perp}
\end{array}
$$

The normalization conditions for the above introduced density matrices are defined from the sum-rule conditions of Eqs. (2.53) and (2.55). For the density matrices of NN SRC, $\rho_{N N}^{N}$, the normalization conditions to satisfy the baryonic and momentum sum rules [36] yields

$$
\int \rho_{N N}^{N}\left(\beta, \mathrm{k}_{\perp}\right) \frac{d \beta}{\beta} d^{2} \mathbf{k}_{\perp}=\int \rho_{N N}^{N}\left(\beta, \mathrm{k}_{\perp}\right) \beta \frac{d \beta}{\beta} d^{2} \mathbf{k}_{\perp}=n_{2 N}^{N},
$$

where $n_{2 N}^{N}$ is the contribution to the total norm of the spectral function. Similar to Eq.(2.37), the light-front density matrix, $\rho_{N N}^{N}$, can be modeled through the high momentum part of the light-front density matrix of the deuteron $\rho_{d}\left(\beta_{1}, k_{1, \perp}\right)$ in the form

$$
\rho_{N N}^{N}\left(\beta_{1}, \mathrm{k}_{1, \perp}\right)=\frac{a_{2}(A, Z)}{\left(2 x_{N}\right)^{\gamma}} \rho_{d}\left(\beta_{1}, \mathrm{k}_{1, \perp}\right) \Theta\left(\mathrm{k}_{1}-\mathrm{k}_{s r c}\right)
$$

where $\mathrm{k}_{1}$ is defined in Eq. (2.80), and $a_{2}(A, Z)$ in Eq. (2.37). A model for the deuteron density matrix $\rho_{d}$ is given in Eq. (3.37).

For the light-front density matrix, $\rho_{C M}$, the conditions of Eq. (2.53) require the following normalization relations:

$$
\begin{gathered}
\int \rho_{C M}\left(\alpha_{N N}, \mathrm{k}_{N N, \perp}\right) \frac{d \alpha_{N N}}{\alpha_{N N}} d^{2} \mathbf{k}_{N N, \perp}=1 \\
\int \rho_{C M}\left(\alpha_{N N}, k_{N N, \perp}\right) \alpha_{N N} \frac{d \alpha_{N N}}{\alpha_{N N}} d^{2} \mathbf{k}_{N N, \perp}=2 .
\end{gathered}
$$


Since in the considered $2 \mathrm{~N}$ SRC model the CM motion is nonrelativistic $\left(\mathbf{k}_{N N} \ll 2 M_{N}\right)$, the momentum distribution $\rho_{C M}$ can be approximated as

$$
\rho_{C M}\left(\alpha_{N N}, \mathrm{k}_{N N, \perp}\right)=\frac{E_{N N} E_{A-2}}{\left(E_{N N}+E_{A-2}\right) / A} \frac{n_{C M}\left(\mathrm{k}_{C M}\right)}{A-\alpha_{N N}} \approx 2 M_{N} n_{C M}\left(\mathrm{k}_{C M}\right),
$$

where $n_{C M}$ is defined in Eq. (2.36). Then, for the "middle" form of (2.102), the normalization condition of Eq. (2.100) is exact, while Eq. (2.101) is exact only in the non-relativistic limit.

The 2N SRC nuclear spectral function in LF approximation [Eq.(2.97)], together with the density matrix for the 2N SRC center of mass [Eq.(2.102)], the density matrix for the relative motion in the 2N SRC [Eq.(2.99)], and the normalization conditions [Eq.(2.98)], [Eq.(2.100)] and [Eq.(2.101)], constitute the mathematical model from which the corresponding computational models and numerical estimates are obtained in the chapters 3 and 4 of the present dissertation.

Finally, it is worth mentioning that in the non-relativistic limit of the density matrix of $2 \mathrm{~N}$ SRC, $\rho\left(\beta_{1}, k_{1, \perp}\right) \approx n_{N N}\left(p_{1}\right) M_{N}$, the 2N SRC LF nuclear spectral function [Eq.(2.97)], similar to the VN approximation, reduces to the SRC model of spectral function of Ref. [52].

\subsubsection{Nuclear spectral function in light-front approximation for three nucleons in short-range correlation}

The calculation of the nuclear spectral function in light-front approximation for three nucleons in short-range correlation starts with the definition of the effective vertex $\hat{V}_{3 N}$ in the $3 \mathrm{~N}$ SRC covariant amplitude (2.11)

$$
\hat{V}_{3 N}=i \bar{a}\left(p_{1}, s_{1}\right) 2 \alpha_{1}^{2} \delta\left(\alpha_{1}+\alpha_{2}+\alpha_{3}-3\right) \delta^{2}\left(\mathbf{p}_{1, \perp}+\mathbf{p}_{2, \perp}+\mathbf{p}_{3, \perp}\right) \delta\left(\tilde{M}_{N}^{2}-\tilde{M}_{N}^{(3 N), 2}\right) a\left(p_{1}, s_{1}\right)
$$

where $\left(\alpha_{2}, \mathbf{p}_{2, \perp}\right),\left(\alpha_{3}, \mathbf{p}_{3, \perp}\right)$ are light-front momentum fractions and transverse momenta of correlated second and third nucleon respectively. The creation, $\bar{a}\left(p_{1}, s_{1}\right)$, and the annihilation, $a\left(p_{1}, s_{1}\right)$, operators of nucleon with four-momentum $p_{1}$ and spin $s_{1}$ satisfy the relations of Eq. 
(2.16). The delta functions represent the momentum and the invariant mass conservation in the vertex.

Since in the light-front $3 \mathrm{~N}$ correlation model, the particles 2 and 3 are considered on lightfront energy shells, the magnitude of the invariant mass $\tilde{M}_{N}^{(3 N), 2}$ is calculated as follows

$$
\begin{aligned}
\tilde{M}_{N}^{(3 N), 2} & =p_{1}^{-} p_{1}^{+}-\mathrm{p}_{1, \perp}^{2}=p_{1+}\left(p_{A-}-p_{2-}-p_{3-}-p_{A-3,-}\right)-\mathrm{p}_{1, \perp}^{2} \\
& =p_{1}^{+}\left(\frac{M_{A}^{2}}{p_{A}^{+}}-\frac{M_{N}^{2}+\mathrm{p}_{2, \perp}^{2}}{p_{2}^{+}}-\frac{M_{N}^{2}+\mathrm{p}_{3, \perp}^{2}}{p_{3}^{+}}-\frac{M_{A-3}^{2}}{p_{A-3}^{+}}\right)-\mathrm{p}_{1, \perp}^{2} \\
& =\frac{p_{1}^{+}}{3 p_{A}^{+}}\left(\frac{M_{A}^{2}}{3}-\frac{M_{N}^{2}+\mathrm{p}_{2, \perp}^{2}}{p_{2}^{+} / 3 p_{A}^{+}}-\frac{M_{N}^{2}+\mathrm{p}_{3, \perp}^{2}}{p_{3}^{+} / 3 p_{A}^{+}}-\frac{M_{A-3}^{2}}{p_{A-3}^{+} / 3 p_{A}^{+}}\right)-\mathrm{p}_{1, \perp}^{2} \\
& =\frac{\alpha_{1}}{3}\left(M_{3 N}^{2}-\frac{M_{N}^{2}+\mathrm{p}_{2, \perp}^{2}}{\alpha_{2} / 3}-\frac{M_{N}^{2}+\mathrm{p}_{3, \perp}^{2}}{\alpha_{3} / 3}\right)-\mathrm{p}_{1, \perp}^{2},
\end{aligned}
$$

with the mass of the 3N SRC defined as:

$$
M_{3 N}^{2}=\frac{3}{A} M_{A}^{2}-3 \frac{M_{A-3}^{2}}{\alpha_{A-3}}
$$

where $\alpha_{A-3}=A-3$.

Similarly to the propagator integration described in section 2.4.2, the integrations by $d p_{2}^{-}$ and $d p_{3}^{-}$in Eq. (2.11) are performed through the " - " components of the positive poles of the propagators of the on-shell particles 2 and 3 respectively, provided that their " + " components are large and positive, so that the $Z$ diagram in this scheme will be suppressed by the inverse power of large " + " component of the nucleon's four-momentum [25]. The integration by $d p_{2}^{-}$ of the propagator of the on-shell particle 2 yields

$$
\begin{aligned}
\oint \frac{d^{4} p_{2}}{p_{2}^{2}-M_{N}+i \varepsilon} & =\oint \frac{d p_{2}^{-} d p_{2}^{+} d^{2} \mathbf{p}_{2, \perp}}{2\left(p_{2}^{+} p_{2}^{-}-\mathrm{p}_{2, \perp}^{2}-M_{N}\right)+i \varepsilon} \\
& =\oint \frac{d p_{2}^{-} d p_{2}^{+} d^{2} \mathbf{p}_{2, \perp}}{2 p_{2}^{+}\left[p_{2}^{-}-\left(\mathrm{p}_{2, \perp}^{2}+M_{N}\right) / p_{2}^{+}\right]+i \varepsilon}=-\left.\frac{2 \pi i d p_{2}^{+} d^{2} \mathbf{p}_{2, \perp}}{2 p_{2}^{+}}\right|_{p_{2}^{-}=\frac{\mathrm{p}_{2, \perp}^{2}+M_{N}}{p_{2}^{+}}} \\
& =-\left.\pi i \frac{d \alpha_{2}}{\alpha_{2}} d^{2} \mathbf{p}_{2, \perp}\right|_{p_{2}^{-}=\frac{\mathrm{p}_{2, \perp}^{2}+M_{N}}{p_{2}^{+}}}
\end{aligned}
$$


and integration by $d p_{3}^{-}$of the propagator of the on-shell particle 3 yields

$$
\begin{aligned}
\oint \frac{d^{4} p_{3}}{p_{3}^{2}-M_{N}+i \varepsilon} & =\oint \frac{d p_{3}^{-} d p_{3}^{+} d^{2} \mathbf{p}_{3, \perp}}{2\left(p_{2}^{+} p_{3}^{-}-\mathrm{p}_{3, \perp}^{2}-M_{N}\right)+i \varepsilon} \\
& =\oint \frac{d p_{3}^{-} d p_{3}^{+} d^{2} \mathbf{p}_{3, \perp}}{2 p_{3}^{+}\left[p_{2}^{-}-\left(\mathrm{p}_{3, \perp}^{2}+M_{N}\right) / p_{3}^{+}\right]+i \varepsilon}=-\left.\frac{2 \pi i d p_{3}^{+} d^{2} \mathbf{p}_{3, \perp}}{2 p_{3}^{+}}\right|_{p_{3}^{-}=\frac{\mathrm{p}_{3, \perp}^{2}+M_{N}}{p_{3}^{+}}} \\
& =-\left.\pi i \frac{d \alpha_{3}}{\alpha_{3}} d^{2} \mathbf{p}_{3, \perp}\right|_{p_{3}^{-}=\frac{\mathrm{p}_{3, \perp}^{2}+M_{N}}{p_{2}^{+}}}
\end{aligned}
$$

The above integrations project the intermediate state to the positive light-front energy state thus excluding the contribution from the Z graph of Fig. 2.1(c). With the diminished contribution from the $Z$ graph, the covariant 3N SRC amplitude [Eq. (2.11)], will result in the light-front spectral function, $P_{A, 3 N}^{N}\left(\alpha_{1}, p_{1, \perp}, \tilde{M}_{N}^{2}\right)$ of the 3N SRC.

As in the LF 2N SRC approximation, the numerator of the propagator for the on-shell particles 2 and 3 in Eq. (2.11), can be expressed by the following on-shell sum rule

$$
\begin{aligned}
& \not p_{2}+M_{N}=\sum_{s_{2}} u\left(p_{2}, s_{2}\right) \bar{u}\left(p_{2}, s_{2}\right), \\
& \not p_{3}+M_{N}=\sum_{s_{3}} u\left(p_{3}, s_{3}\right) \bar{u}\left(p_{3}, s_{3}\right),
\end{aligned}
$$

where $u\left(p_{i}, s_{i}\right)$ is the spinor of the particle $i$ with momentum $p_{i}$ and spin projection $s_{i}$. Using a similar approximate relation for the $2^{\prime}$ propagator which represents the nucleon between consecutive $N N$ interaction vertices yields

$$
\not p_{2^{\prime}}+M_{N}=\sum_{s_{2^{\prime}}} u\left(p_{2^{\prime}}, s_{2^{\prime}}\right) \bar{u}\left(p_{2^{\prime}}, s_{2^{\prime}}\right)
$$

Inserting Eqs. (2.16) and (2.106) -(2.110) in the covariant 3N SRC amplitude [Eq. (2.11)], and summing over all possible spin projections $s_{2}, s_{3}$ and $s_{2^{\prime}}$, reduces the latter to the $3 \mathrm{~N}$ SRC 
part of the nuclear spectral function in LF approximation, namely

$$
\begin{aligned}
P_{A, 3 N}^{N}\left(\alpha_{1}, \mathrm{p}_{1, \perp}, \tilde{M}_{N}^{2}\right) & =\sum_{s_{2}, s_{3}, s_{2^{\prime}}, \tilde{s}_{2^{\prime}}} \int \bar{u}\left(k_{1}, \lambda_{1}\right) \bar{u}\left(k_{2}, \lambda_{2}\right) \bar{u}\left(k_{3}, \lambda_{3}\right) \Gamma_{N N \rightarrow N, N}^{\dagger} \frac{u\left(p_{2^{\prime}}, \tilde{s}_{2^{\prime}}\right) \bar{u}\left(p_{2^{\prime}}, \tilde{s}_{2^{\prime}}\right)}{p_{2^{\prime}}^{2}-M_{N}^{2}} \Gamma_{N N \rightarrow N, N}^{\dagger} \\
& \times 2 \alpha_{1}^{2} \delta\left(\alpha_{1}+\alpha_{2}+\alpha_{3}-3\right) \delta^{2}\left(\mathbf{p}_{1, \perp}+\mathbf{p}_{2, \perp}+\mathbf{p}_{3, \perp}\right) \delta\left(\tilde{M}_{N}^{2}-\tilde{M}_{N}^{(3 N), 2}\right) \\
& \times \frac{u\left(p_{1}, s_{1}\right)}{p_{1}^{2}-M_{N}^{2}} u\left(p_{2}, s_{2}\right) \bar{u}\left(p_{2}, s_{2}\right) \frac{\bar{u}\left(p_{1}, s_{1}\right)}{p_{1}^{2}-M_{N}^{2}} \Gamma_{N N \rightarrow N, N} \frac{u\left(p_{2^{\prime}}, s_{2^{\prime}}\right) \bar{u}\left(p_{2^{\prime}}, s_{2^{\prime}}\right)}{p_{2^{\prime}}^{2}-M_{N}^{2}} \\
& \times u\left(p_{3}, s_{3}\right) \bar{u}\left(p_{3}, s_{3}\right) \Gamma_{N N \rightarrow N N}^{\dagger} u\left(k_{1}, \lambda_{1}\right) u\left(k_{2}, \lambda_{2}\right) u\left(k_{3}, \lambda_{3}\right) \\
& \times \frac{d \alpha_{2}}{\alpha_{2}} \frac{d^{2} \mathbf{p}_{2, \perp}}{2(2 \pi)^{3}} \frac{d \alpha_{3}}{\alpha_{3}} \frac{d^{2} \mathbf{p}_{3, \perp}}{2(2 \pi)^{3}}
\end{aligned}
$$

\section{Light-front wave function of the first 2N SRC}

The calculation of the light-front wave function of the first 2N SRC, shown in Fig. 2.3, between particle 2 with momentum $k_{2}$, and particle 3 with momentum $k_{3}$, which results in particle $2^{\prime}$ with momentum $p_{2^{\prime}}$, and particle 3 with momentum $p_{3}$, depends on the consideration of the following expression, as well as its complex conjugated form, in Eq. (2.111):

$$
\frac{\bar{u}\left(p_{2^{\prime}}, s_{2^{\prime}}\right) \bar{u}\left(p_{3}, s_{3}\right) \Gamma_{N N \rightarrow N, N} u\left(k_{2}, \lambda_{2}\right) u\left(k_{3}, \lambda_{3}\right)}{p_{2^{\prime}}^{2}-M_{N}^{2}} .
$$

Before calculating the LF wave functions, it is convenient to define important kinematic variables and relations that result from the collinear approximation. The assumptions of collinear approximation in the diagram of Fig. 2.3 implies that the momentum fractions of the 3N SRCs carried by each initial nucleon is unity and their total transverse momenta is neglected, then

$$
\begin{aligned}
k^{+} & =k_{1}^{+}+k_{2}^{+}+k_{3}^{+}, \\
\alpha_{k} & =\frac{3 k_{1}^{+}}{k^{+}}=\frac{3 k_{2}^{+}}{k^{+}}=\frac{3 k_{3}^{+}}{k^{+}}=1 \\
\mathbf{k}_{1, \perp}+\mathbf{k}_{2, \perp}+\mathbf{k}_{3, \perp} & =0
\end{aligned}
$$


also from the energy and momentum conservation it follows that:

$$
\begin{gathered}
k_{1}+k_{2}+k_{3}=p_{1}+p_{2}+p_{3}, \\
k^{+}=p_{1}^{+}+p_{2}^{+}+p_{3}^{+}, \\
\mathbf{p}_{1, \perp}+\mathbf{p}_{2, \perp}+\mathbf{p}_{3, \perp}=0, \\
k_{2}+k_{3}=p_{2^{\prime}}+p_{3}, \\
k_{1}+p_{2^{\prime}}=p_{2}+p_{3},
\end{gathered}
$$

and the momentum fractions of the initial as well as those of the correlated nucleons are

$$
\begin{aligned}
\alpha_{i} & =\frac{3 p_{i}^{+}}{k^{+}} \quad i=1,2,3 \\
\alpha_{1}+\alpha_{2}+\alpha_{3} & =3 \\
\alpha_{2^{\prime}} & =\frac{3 p_{2^{\prime}}^{+}}{k^{+}}=\frac{3\left(k_{2}+k_{3}-p_{3}\right)}{k^{+}}=2-\alpha_{3}, \\
\beta_{1} & =\frac{2 \alpha_{1}}{\alpha_{12}}=\frac{2 \alpha_{1}}{\alpha_{1}+\alpha_{2}}=\frac{2 \alpha_{1}}{3-\alpha_{3}}, \\
\beta_{2} & =\frac{2 \alpha_{2}}{\alpha_{12}}=\frac{2 \alpha_{2}}{\alpha_{1}+\alpha_{2}}=\frac{2 \alpha_{2}}{3-\alpha_{3}}, \\
\beta_{3} & =\frac{2 \alpha_{3}}{\alpha_{2^{\prime} 3}}=\frac{2 \alpha_{3}}{\alpha_{2^{\prime}}+\alpha_{3}}=\alpha_{3}, \\
\beta_{2^{\prime}} & =\frac{2 \alpha_{2^{\prime}}}{\alpha_{2^{\prime} 3}}=\frac{2\left(2-\alpha_{3}\right)}{2}=2-\alpha_{3} .
\end{aligned}
$$

By applying the above defined relations, as well as the light-front momentum and energy conservation at the $\Gamma_{N N \rightarrow N, N}$ vertex, and the on-shell condition of initial particles as well of the 
correlated particles 2 and 3, the propagator in Eq. (2.112) can be expressed as

$$
\begin{aligned}
p_{2^{\prime}}^{2}-M_{N}^{2} & =\left(k_{2}+k_{3}-p_{3}\right)^{2}-M_{N}^{2} \\
& \left.=\left(k_{2}^{+}+k_{3}^{+}-p_{3}^{+}\right)\left(k_{2}^{-}+k_{3}^{-}-p_{3}^{-}\right)-\mathrm{p}_{3, \perp}^{2}\right)-M_{N}^{2} \\
& =p_{2^{\prime}}^{+}\left(\frac{M_{N}^{2}}{k_{2}^{+}}+\frac{M_{N}^{2}}{k_{3}^{+}}-\frac{M_{N}^{2}+\mathrm{p}_{3, \perp}^{2}}{p_{3}^{+}}-\frac{M_{N}^{2}+\mathrm{p}_{3, \perp}^{2}}{p_{2^{\prime}}^{+}}\right) \\
& =\left(2-\alpha_{3}\right)\left(2 M_{N}^{2}-\frac{M_{N}^{2}+\mathrm{p}_{3, \perp}^{2}}{\alpha_{3}}-\frac{M_{N}^{2}+\mathrm{p}_{3, \perp}^{2}}{2-\alpha_{3}}\right) \\
& =\frac{2-\beta_{3}}{2}\left[M_{23}^{2}-4 \frac{M_{N}^{2}+\mathrm{p}_{3, \perp}^{2}}{\beta_{3}\left(2-\beta_{3}\right)}\right]
\end{aligned}
$$

where the invariant mass $M_{23}^{2}$ squared is

$$
\begin{aligned}
M_{23}^{2} & =\left(k_{2}+k_{3}\right)^{2}=\left(k_{2}^{+}+k_{3}^{+}\right)\left(k_{2}^{-}+k_{3}^{-}\right) \\
& =M_{N}^{2}\left(2+\frac{k_{3}^{+}}{k_{2}^{+}}+\frac{k_{2}^{+}}{k_{3}^{+}}\right) \approx 4 M_{N}^{2} .
\end{aligned}
$$

The propagator (2.116) can be completely expressed though the internal momenta of the $N N$ system by introducing the relative momentum, $\tilde{\mathbf{k}}_{3}$, in the $N N$ center of mass frame, as follows:

$$
\tilde{\mathrm{k}}_{3}^{2}=\frac{M_{N}^{2}+\mathrm{p}_{3, \perp}^{2}}{\beta_{3}\left(2-\beta_{3}\right)}-M_{N}^{2} .
$$

The relative momentum $\tilde{\mathbf{k}}_{3}$ is invariant with respect to the Lorentz boost in the $\mathbf{p}_{\mathbf{N N}}$ direction, and will be used to set a momentum scale for the $2 \mathrm{~N}$ SRCs, requiring $\tilde{\mathrm{k}}_{3} \geq \mathrm{k}_{s r c}$ similar to Eq. (2.37).

The propagator (2.116) can therefore be expressed as

$$
p_{2^{\prime}}^{2}-M_{N}^{2}=\frac{2-\beta_{3}}{2}\left[M_{23}^{2}-4\left(M_{N}^{2}+\tilde{\mathrm{k}}_{3}^{2}\right)\right]
$$


Inserting Eq. (2.119) in Eq. (2.112), the light-front wave function of the first $N N$ SRC is defined as

$$
\psi_{N N}\left(\beta_{2^{\prime}}, \mathrm{p}_{3, \perp}, s_{2^{\prime}}, s_{3}\right)=\frac{1}{\sqrt{2(2 \pi)^{3}}} \frac{\bar{u}\left(p_{2^{\prime}}, s_{2^{\prime}}\right) \bar{u}\left(p_{3}, s_{3}\right) \Gamma_{N N \rightarrow N, N} u\left(k_{2}, \lambda_{2}\right) u\left(k_{3}, \lambda_{3}\right)}{\frac{1}{2}\left[M_{23}^{2}-4\left(M_{N}^{2}+\tilde{\mathrm{k}}_{3}^{2}\right)\right]} .
$$

The above defined wave function results in the following simplified expression for Eq.(2.112)

$$
\frac{\bar{u}\left(p_{2^{\prime}}, s_{2^{\prime}}\right) \bar{u}\left(p_{3}, s_{3}\right) \Gamma_{N N \rightarrow N N} u\left(k_{2}\right) u\left(k_{3}\right)}{p_{2^{\prime}}^{2}-M_{N}^{2}}=\sqrt{2(2 \pi)^{3}} \frac{\psi_{N N}\left(\beta_{2^{\prime}}, \mathrm{p}_{3, \perp}, s_{2^{\prime}}, s_{3}\right)}{2-\beta_{3}} .
$$

\section{Light-front wave function of the second 2N SRC}

The calculation of the light-front wave function of the second 2N SRC, shown in Fig. 2.3, between particle 1 with momentum $k_{1}$, and particle $2^{\prime}$ with momentum $p_{2^{\prime}}$, which results in particle 1 with momentum $p_{1}$, and particle 2 with momentum $p_{2}$, depends on the consideration of the following expression, as well as its complex conjugated form, in Eq. (2.111):

$$
\frac{\bar{u}\left(p_{1}, s_{1}\right) \bar{u}\left(p_{2}, s_{2}\right) \Gamma_{N N \rightarrow N, N} u\left(p_{2^{\prime}}, s_{2^{\prime}}\right) u\left(k_{1}, \lambda_{1}\right)}{p_{1}^{2}-M_{N}^{2}} .
$$

By applying the relations in Eqs. (2.113) to (2.115), as well as the light-front momentum and energy conservation at the $\Gamma_{N N \rightarrow N, N}$ vertex, and the on-shell condition of the initial particles as well of the correlated particles 2 and 3, the propagator in Eq. (2.122) can be expressed as

$$
\begin{aligned}
p_{1}^{2}-M_{N}^{2} & =\left(k_{1}+k_{2}+k_{3}-p_{2}-p_{3}\right)^{2}-M_{N}^{2} \\
& =\left(k_{1}^{+}+k_{2}^{+}+k_{3}^{+}-p_{2}^{+}-p_{3}^{+}\right)\left(k_{1}^{-}+k_{2}^{-}+k_{3}^{-}-p_{2}^{-}-p_{3}^{-}\right)-\left(\mathbf{p}_{2, \perp}+\mathbf{p}_{3, \perp}\right)^{2}-M_{N}^{2} \\
& =p_{1}^{+}\left(\frac{M_{N}^{2}}{k_{1}^{+}}+\frac{M_{N}^{2}}{k_{2}^{+}}+\frac{M_{N}^{2}}{k_{3}^{+}}-\frac{M_{N}^{2}+\mathrm{p}_{2, \perp}^{2}}{p_{2}^{+}}-\frac{M_{N}^{2}+\mathrm{p}_{3, \perp}^{2}}{p_{3}^{+}}-\frac{M_{N}^{2}+\mathrm{p}_{1, \perp}^{2}}{p_{1}^{+}}\right) \\
& =\alpha_{1}\left(3 M_{N}^{2}-\frac{M_{N}^{2}+\mathrm{p}_{2, \perp}^{2}}{\alpha_{2}}-\frac{M_{N}^{2}+\mathrm{p}_{3, \perp}^{2}}{\alpha_{3}}-\frac{M_{N}^{2}+\mathrm{p}_{1, \perp}^{2}}{\alpha_{1}}\right) \\
& =\frac{\beta_{1}}{2}\left[M_{12}^{2}-\frac{4\left[M_{N}^{2}+\left(\mathbf{p}_{1, \perp}-\frac{\beta_{1}}{2} \mathbf{p}_{12, \perp}\right)^{2}\right]}{\left(2-\beta_{1}\right) \beta_{1}}\right],
\end{aligned}
$$


where $\mathbf{p}_{\mathbf{1 2 , \perp}}=\mathbf{p}_{\mathbf{1}, \perp}+\mathbf{p}_{\mathbf{2}, \perp}$, and the invariant mass $M_{12}^{2}$ squared is

$$
\begin{aligned}
M_{12}^{2} & =\left(k_{1}+k_{2}+k_{3}-p_{3}\right)^{2} \\
& =\left(k_{1}^{+}+k_{2}^{+}+k_{3}^{+}-p_{3}^{+}\right)\left(k_{1}^{-}+k_{2}^{-}+k_{3}^{-}-p_{3}^{-}\right)-\mathrm{p}_{3, \perp}^{2} \\
& =\left(1-\frac{\alpha_{3}}{3}\right)\left(9 M_{N}^{2}-\frac{M_{N}^{2}+\mathrm{p}_{3, \perp}^{2}}{\alpha_{3} / 3}\right)-\mathrm{p}_{3, \perp}^{2} \\
& \approx M_{3 N}^{2}\left(1-\frac{\alpha_{3}}{3}\right)-3 \frac{M_{N}^{2}+\mathrm{p}_{3, \perp}^{2}}{\alpha_{3}}+M_{N}^{2} .
\end{aligned}
$$

The propagator (2.123) can be completely expressed though the internal momenta of the $N N$ system by introducing the relative momentum, $\tilde{\mathbf{k}}_{1}$, in the $N N$ center of mass frame, as follows:

$$
\tilde{\mathrm{k}}_{1}^{2}=\frac{M_{N}^{2}+\tilde{\mathrm{k}}_{1, \perp}^{2}}{\beta_{1}\left(2-\beta_{1}\right)}-M_{N}^{2} \text {, }
$$

where the relative transverse momentum is

$$
\tilde{\mathbf{k}}_{1, \perp}=\mathbf{p}_{1, \perp}-\frac{\beta_{\mathbf{1}}}{\mathbf{2}} \mathbf{p}_{12, \perp}
$$

The relative momentum $\tilde{\mathbf{k}}_{1}$ is invariant with respect to the Lorentz boost in the $\mathbf{p}_{\mathbf{N N}}$ direction, and will be used to set a momentum scale for the $2 \mathrm{~N}$ SRCs, requiring $\tilde{\mathrm{k}}_{1} \geq \mathrm{k}_{s r c}$ similar to Eq. (2.37).

The propagator (2.123) can therefore be expressed as

$$
\begin{aligned}
p_{1}^{2}-M_{N}^{2} & =\frac{\beta_{1}}{2}\left[M_{12}^{2}-\frac{4\left(M_{N}^{2}+\mathrm{k}_{1, \perp}^{2}\right)}{\left(2-\beta_{1}\right) \beta_{1}}\right] \\
& =\frac{\beta_{1}}{2}\left[M_{12}^{2}-4\left(M_{N}^{2}+\tilde{\mathrm{k}}_{1}^{2}\right)\right] .
\end{aligned}
$$


Inserting Eq. (2.127) in Eq. (2.122), the light-front wave function of the second $N N$ SRC is defined as

$$
\psi_{N N}\left(\beta_{1}, \tilde{\mathrm{k}}_{1, \perp}, s_{1}, s_{2}\right)=\frac{1}{\sqrt{2(2 \pi)^{3}}} \frac{\bar{u}\left(p_{1}, s_{1}\right) \bar{u}\left(p_{2}, s_{2}\right) \Gamma_{N N \rightarrow N, N} u\left(p_{2^{\prime}}, s_{2^{\prime}}\right) u\left(k_{1}, \lambda_{1}\right)}{\frac{1}{2}\left[M_{12}^{2}-4\left(M_{N}^{2}+\tilde{\mathrm{k}}_{1}^{2}\right)\right]} .
$$

The above defined wave function results in the following simplified expression for Eq.(2.122)

$$
\frac{\bar{u}\left(p_{1}, s_{1}\right) \bar{u}\left(p_{2}, s_{2}\right) \Gamma_{N N \rightarrow N, N} u\left(p_{2^{\prime}}, s_{2^{\prime}}\right) u\left(k_{1}\right)}{p_{1}^{2}-M_{N}^{2}}=\sqrt{2(2 \pi)^{3}} \frac{\psi_{N N}\left(\beta_{1}, \tilde{\mathrm{k}}_{1, \perp}, s_{1}, s_{2}\right)}{\beta_{1}}
$$

\section{N SRC light-front nuclear spectral function}

Inserting Eqs. (2.121) and (2.129) in Eq.(2.111), the following expression for the 3N SRC lightfront nuclear spectral function is obtained

$$
\begin{aligned}
P_{A, 3 N}^{N}\left(\alpha_{1}, \mathrm{p}_{1, \perp}, \tilde{M}_{N}^{2}\right) & =\sum_{s_{1}, s_{2}, s_{3}, s_{2^{\prime}}} \int \frac{\psi_{N N}^{\dagger}\left(\beta_{2^{\prime}}, \mathrm{p}_{3, \perp}, s_{2^{\prime}}, s_{3}\right)}{2-\beta_{3}} \frac{\psi_{N N}^{\dagger}\left(\beta_{1}, \tilde{\mathrm{k}}_{1, \perp}, s_{1}, s_{2}\right)}{\beta_{1}} \\
& \times 2 \alpha_{1}^{2} \delta\left(\alpha_{1}+\alpha_{2}+\alpha_{3}-3\right) \delta^{2}\left(\mathbf{p}_{1, \perp}+\mathbf{p}_{2, \perp}+\mathbf{p}_{3, \perp}\right) \delta\left(\tilde{M}_{N}^{2}-\tilde{M}_{N}^{(3 N), 2}\right) \\
& \times \frac{\psi_{N N}\left(\beta_{1}, \tilde{\mathrm{k}}_{1, \perp}, s_{1}, s_{2}\right)}{\beta_{1}} \frac{\psi_{N N}\left(\beta_{2^{\prime}}, \mathrm{p}_{3, \perp}, s_{2^{\prime}}, s_{3}\right)}{2-\beta_{3}} \frac{d \alpha_{2}}{\alpha_{2}} d^{2} \mathbf{p}_{2, \perp} \frac{d \alpha_{3}}{\alpha_{3}} d^{2} \mathbf{p}_{3, \perp}
\end{aligned}
$$

The nuclear spectral function (2.130) can be further simplified by summing over the final and average of all possible initial polarization configurations, similar to the one in VN approximation [Eq. (2.33)], as well as by introducing the light-front spin averaged density matrices for the relative motion in the first and second 2N SRC, similar to those defined in Eq.(2.95). Then, the 3N SRC light-front nuclear spectral function is obtained as the convolution of two NN light-front density matrices as follows:

$$
\begin{aligned}
P_{A, 3 N}^{N}\left(\alpha_{1}, \mathrm{p}_{1, \perp}, \tilde{M}_{N}^{2}\right) & =\int \frac{3-\alpha_{3}}{2\left(2-\alpha_{3}\right)^{2}} \rho_{N N}\left(\beta_{2^{\prime}}, \mathrm{p}_{3, \perp}\right) \rho_{N N}\left(\beta_{1}, \tilde{\mathrm{k}}_{1, \perp}\right) 2 \delta\left(\alpha_{1}+\alpha_{2}+\alpha_{3}-3\right) \\
& \times \delta^{2}\left(\mathbf{p}_{1, \perp}+\mathbf{p}_{2, \perp}+\mathbf{p}_{3, \perp}\right) \delta\left(\tilde{M}_{N}^{2}-\tilde{M}_{N}^{(3 N), 2}\right) d \alpha_{2} d^{2} \mathbf{p}_{2, \perp} d \alpha_{3} d^{2} \mathbf{p}_{3, \perp}
\end{aligned}
$$


The normalization conditions for the above introduced density matrices are defined from the sum-rule conditions of Eqs. (2.53) and (2.55). For the density matrices of NN SRC, $\rho_{N N}^{N}$, the normalization conditions to satisfy the baryonic and momentum sum rules [36] yields

$$
\int \rho_{N N}^{N}\left(\beta, \mathrm{k}_{\perp}\right) \frac{d \beta}{\beta} d^{2} \mathbf{k}_{\perp}=\int \rho_{N N}^{N}\left(\beta, \mathrm{k}_{\perp}\right) \beta \frac{d \beta}{\beta} d^{2} \mathbf{k}_{\perp}=n_{3 N}^{N},
$$

where $n_{3 N}^{N}$ is the contribution to the total norm of the nuclear spectral function.

In the nuclear spectral function [Eq.(2.131)], similar to Eq. (2.49) for VN approximation, the product of the two density matrices is expressed through the product of high momentum parts of the deuteron density matrices in the form:

$\rho_{N N}\left(\beta_{2^{\prime}}, \mathrm{p}_{3, \perp}\right) \rho_{N N}\left(\beta_{1}, \tilde{\mathrm{k}}_{1, \perp}\right)=a_{2}^{2}(A, Z) C^{N}(A, Z) \rho_{d}\left(\beta_{2^{\prime}}, \mathrm{p}_{3, \perp}\right) \Theta\left(\tilde{\mathrm{k}}_{1}-k_{s r c}\right) \rho_{d}\left(\beta_{1}, \tilde{\mathrm{k}}_{1, \perp}\right) \Theta\left(\tilde{k}_{3}-k_{s r c}\right)$,

where $\tilde{k}_{1}$ and $\tilde{k}_{3}$ are defined in Eqs. (2.125) and (2.118) respectively. The factors $a_{2}(A, Z)$ and $C^{N}(A, Z)$ are the same as those defined in Eq. (2.49) for the case of VN approximation. A model for the deuteron density matrix $\rho_{d}$ is given in Eq. (3.37).

The 3N SRC nuclear spectral function in LF approximation [Eq.(2.131)], together with the product of the density matrices for the relative momentum distribution of the NN SRC [Eq.(2.133)], as well as the normalization conditions [Eq.(2.132)], constitute the mathematical model from which the corresponding computational models and numerical estimates are obtained in the chapters 3 and 4 of the present dissertation.

\subsection{Summary.}

On the basis of the NN short-range correlation picture of the high-momentum component of nuclear wave function, a model has been developed for the nuclear spectral functions in the domain of high momentum and high removal energy of bound nucleon in the nucleus. In the high momentum domain, the models of nuclear spectral functions should be relativistic.

A main focus therefore in developing the models, in the present chapter, is given to the treatment of the relativistic effects which are important for the bound nucleon momenta ex- 
ceeding the characteristic Fermi momentum, $k_{F}$, in the nucleus. The relativistic effects in the present dissertation are treated by using the effective Feynman diagrammatic approach, in which the Lorentz covariant amplitudes are reduced to the nuclear spectral functions, in a process of calculation designed to trace the relativistic effects entering in such functions.

One of the main ambiguities related to the treatment of relativistic effects is the account for the vacuum fluctuations ( $Z$ graphs) which ultimately alter the definition of the spectral function as a probability of finding a bound nucleon in the nucleus with the given momentum and positive removal energy. Two approaches are employed in the present dissertation to deal with the vacuum fluctuations: virtual nucleon and light-front approximations, both suitable for an adequate treatment of the corresponding relativistic effects.

The results obtained in the present chapter within VN [Eq. (2.34)], and LF [Eq. (2.97)], represent an attempt to account for the relativistic effects in the domain of $2 \mathrm{~N}$ SRCs with center of mass motion of the NN pair. The results agree with the 2N SRC (with center of mass motion) model of Ref. [52] in the non-relativistic limit.

The above defined approaches to deal with the vacuum fluctuations are extended to calculate the contributions from three-nucleon short-range correlations. The 3N SRCs are modeled as two sequential NN short-range correlations. The corresponding models of nuclear spectral functions are based on the collinear approach in which a negligible center of mass momentum for the residual or uncorrelated $A-3$ nuclear system is assumed. The derived spectral functions within $\mathrm{VN}$ [Eq. (2.47)] and LF [Eq. (2.131)] approximations represent the results for 3N SRC contribution to the nuclear spectral functions.

The models for nuclear spectral function developed in the present chapter also account for the experimental results of the strong (by factor of 20) dominance of the $p n$ SRCs in nuclei, as compared to the $p p$ and $n n$ correlations, for internal momentum range of $\sim 250-$ $650 \mathrm{MeV} / c$. The dominance of the pn correlations implies that to quantitatively describe the obtained models of nuclear spectral functions, in a high momentum domain, only what is needed is the knowledge of the high momentum deuteron wave function, either in the laboratory frame (for $\mathrm{VN}$ approximation) or on the light front (for LF approximation). 


\section{CHAPTER 3}

\section{Multinucleon short-range correlation model for nuclear spectral functions: Models for numerical computation}

In order to obtain numerical estimates of the nuclear spectral functions, the computational models are developed in the present chapter by integration of the delta functions in the mathematical models obtained in chapter 2 . The integrals are calculated by using the following formulas [83]

$$
\int f(x) \delta(g(x)) d x=\sum_{a} \int f(x) \frac{\delta\left(x-x^{a}\right)}{\left|g^{\prime}\left(x^{a}\right)\right|_{x=x^{a}}} d x=\sum_{a} \frac{f\left(x^{a}\right)}{\left|g^{\prime}\left(x^{a}\right)\right|_{x=x^{a}}} .
$$

where $x^{a}$ are the roots of $g(x)=0$.

The prediction that single proton or neutron momentum distributions in the $2 \mathrm{~N}$ SRC domain are inversely proportional to their relative fractions in nuclei Ref. [104,112], is represented in the computational models of the nuclear spectral functions by the proton and neutron relative fraction factor $x_{p / n}$, introduced in Eq. (2.37) and given by:

$$
\begin{aligned}
& x_{p}=\frac{Z}{A}, \\
& x_{n}=\frac{A-Z}{A} .
\end{aligned}
$$

In the case of $3 \mathrm{~N}$ SRC for asymmetric nuclei, the $3 \mathrm{~N}$ SRC suppression factor $C^{N}(A, Z)$, defined in Eq. (2.49), which accounts for the effects associated with the isospin structure of two-nucleon recoil system, are given by

$$
\begin{aligned}
& C^{p}(A, Z)=\frac{2(Z-1)}{A}, \\
& C^{n}(A, Z)=\frac{2(A-Z-1)}{A} .
\end{aligned}
$$

The computational models developed in the present chapter for 2N SRC and 3N SRC in asymmetric nuclei are specifically associated to proton or to neutron through the factors given by Eqs. (3.2) and (3.3) respectively. 
The outline of the chapter is as follows. Section 3.1 presents the computational models for $2 \mathrm{~N}$ and $3 \mathrm{~N}$ SRC nuclear spectral functions in $\mathrm{VN}$ approximation. The computational models for $2 \mathrm{~N}$ and $3 \mathrm{~N}$ SRC nuclear spectral functions in LF approximation are developed in section 3.2. Section 3.3 includes the computational models for $2 \mathrm{~N}$ and $3 \mathrm{~N}$ SRC nuclear spectral functions in the non-relativistic limit of the VN approximation. Section 3.4 summarizes the results of the chapter.

\subsection{Computational models for SRC nuclear spectral function in virtual nucleon approximation}

The computational models for high momentum nuclear spectral function in virtual nucleon (VN) approximation, with two and three nucleons in short range correlation, are developed in the present section.

\subsubsection{Computational model for two nucleon SRC nuclear spectral function in virtual nucleon approximation}

The following expression for the 2N SRC nuclear spectral function in VN approximation is obtained by inserting the relative momentum distribution of the NN SRC [Eq.(2.37)] in Eq. (2.34), namely

$$
S_{A, 2 N}^{p / n}\left(\mathrm{p}_{1}, E_{m}\right)=\frac{a_{2}(A, Z)}{\left(2 x_{p / n}\right)^{\gamma}} \int \frac{n_{d}\left(\mathrm{p}_{\mathrm{rel}}\right) \Theta\left(\mathrm{p}_{\mathrm{rel}}-\mathrm{k}_{\mathrm{src}}\right)}{\frac{M_{N}-E_{m}-T_{A-1}}{M_{A} / A}} n_{C M}\left(\mathrm{p}_{\mathrm{NN}}\right) \delta\left[E_{m}-E_{m}^{2 N}\right] d \mathbf{p}_{\mathrm{NN}},
$$

where $p / n$ indicates proton and neutron respectively, the parameter $a_{2}(A, Z)$ is defined in Eq. (2.37), $x_{p / n}$ are given by Eq. (3.2), and $n_{d}$ is the deuteron momentum distribution calculated from a nonrelativistic wave function. 
The relativistic kinetic energy of the on-shell correlated nucleon 2 [Fig. 2.2 (b)], is given by

$$
\begin{aligned}
T_{2} & =E_{2}-M_{N}=\sqrt{\mathrm{p}_{2}^{2}+M_{N}^{2}}-M_{N} \\
& =\sqrt{\left(\mathbf{p}_{N N}-\mathbf{p}_{1}\right)^{2}+M_{N}^{2}}-M_{N} \\
& =\sqrt{\mathrm{p}_{1}^{2}-2 \mathrm{p}_{1} \mathrm{p}_{\mathrm{NN}} \cos \theta_{1 \mathrm{NN}}+\mathrm{p}_{\mathrm{NN}}^{2}+M_{N}^{2}}-M_{N},
\end{aligned}
$$

where $\theta_{1 \mathrm{NN}}$ is the angle between $\mathbf{p}_{1}$ and $\mathbf{p}_{\mathrm{NN}}$. Hence, the removal energy $E_{m}^{2 N}$ for the $2 \mathrm{~N}$ SRC [Eq. (2.23)] can be expressed as

$$
E_{m}^{2 N}=E_{t h r}^{(2)}+\sqrt{\mathrm{p}_{1}^{2}-2 \mathrm{p}_{1} p_{N N} \cos \theta_{1 N N}+\mathrm{p}_{N N}^{2}+M_{N}^{2}}-M_{N}+\frac{\mathrm{p}_{\mathrm{NN}}^{2}}{2 M_{A-2}}-\frac{\mathrm{p}_{1}^{2}}{2 M_{A-1}}
$$

and the argument of the delta function in the nuclear spectral function [Eq.(3.4)] is therefore

$$
E_{m}-E_{m}^{2 N}=E_{m}-E_{t h r}^{(2)}+M_{N}-\sqrt{\mathrm{p}_{1}^{2}-2 \mathrm{p}_{1} \mathrm{p}_{\mathrm{NN}} \cos \theta_{1 \mathrm{NN}}+\mathrm{p}_{\mathrm{NN}}+M_{N}^{2}}-\frac{\mathrm{p}_{\mathrm{NN}}^{2}}{2 M_{A-2}}+\frac{\mathrm{p}_{1}^{2}}{2 M_{A-1}}
$$

where $E_{t h r}^{(2)} \approx 2 M_{N}+M_{A-2}-M_{A}$.

If $\cos \tilde{\theta}_{1 \mathrm{NN}}$ is the root of $E_{m}-E_{m}^{2 N}=0$, it follows that

$$
E_{20}=\sqrt{\mathrm{p}_{1}^{2}-2 \mathrm{p}_{1} \mathrm{p}_{\mathrm{NN}} \cos \tilde{\theta}_{1 \mathrm{NN}}+\mathrm{p}_{\mathrm{NN}}+M_{N}^{2}}=E_{m}-E_{t h r}^{(2)}+M_{N}-\frac{\mathrm{p}_{\mathrm{NN}}^{2}}{2 M_{A-2}}+\frac{\mathrm{p}_{1}^{2}}{2 M_{A-1}},
$$

where $E_{20}$ is the relativistic energy of the nucleon 2 for $\cos \tilde{\theta}_{1 \mathrm{NN}}$.

$\cos \tilde{\theta}_{1 \mathrm{NN}}$ is then given by

$$
\cos \tilde{\theta}_{1 \mathrm{NN}}=\frac{\mathrm{p}_{1}^{2}+\mathrm{p}_{\mathrm{NN}}^{2}+M_{N}^{2}-E_{20}^{2}}{2 \mathrm{p}_{1} \mathrm{p}_{\mathrm{NN}}}
$$

with the restrictions

$$
\begin{aligned}
E_{20}^{2} & \geq 0 \\
\cos ^{2} \tilde{\theta}_{1 \mathrm{NN}} & \leq 1
\end{aligned}
$$


The derivative of Eq. (3.7) yields

$$
\left.\frac{d\left(E_{m}-E_{m}^{2 N}\right)}{d \cos \theta_{1 \mathrm{NN}}}\right|_{\theta_{1 \mathrm{NN}}=\tilde{\theta}_{1 \mathrm{NN}}}=\frac{\mathrm{p}_{1} \mathrm{p}_{\mathrm{NN}}}{\sqrt{\mathrm{p}_{1}^{2}-2 \mathrm{p}_{1} \mathrm{p}_{\mathrm{NN}} \cos \tilde{\theta}_{1 \mathrm{NN}}+\mathrm{p}_{\mathrm{NN}}+M_{N}^{2}}}=\frac{\mathrm{p}_{1} \mathrm{p}_{\mathrm{NN}}}{E_{20}} .
$$

Then, from Eqs.(3.1), and (3.8) to (3.11), the integration of the delta function in the nuclear spectral functions [Eq.(3.4)] over $\cos \theta_{1 \mathrm{NN}}$ yields

$$
\int \delta^{0}\left[E_{m}-E_{m}^{2 N}\right] d \mathbf{p}_{\mathrm{NN}}=2 \pi \int \delta^{0}\left[E_{m}-E_{m}^{2 N}\right] \mathrm{p}_{\mathrm{NN}}^{2} d \mathrm{p}_{\mathrm{NN}} d \cos \theta_{1 \mathrm{NN}}=2 \pi \int \frac{E_{20}}{p_{1}} \mathrm{p}_{\mathrm{NN}} d \mathrm{p}_{\mathrm{NN}}
$$

The 2N SRC nuclear spectral functions [Eq.(3.4)] are therefore given by:

$$
S_{A, 2 N}^{p / n}\left(\mathrm{p}_{1}, E_{m}\right)=2 \pi \frac{a_{2}(A, Z)}{\left(2 x_{p / n}\right)^{\gamma}} \int_{0}^{1} \frac{n_{d}\left(\mathrm{p}_{\mathrm{rel}}\right)}{\omega_{\text {rel }}} n_{C M}\left(\mathrm{p}_{\mathrm{NN}}\right) \frac{E_{20}}{p_{1}} \Theta\left(\mathrm{p}_{\mathrm{rel}}-\mathrm{k}_{\mathrm{src}}\right) \mathrm{p}_{\mathrm{NN}} d \mathrm{p}_{\mathrm{NN}},
$$

where the center of mass momentum distribution is given by Eq.(2.36), and the relative momentum of the correlated nucleons by

$$
\begin{aligned}
\mathrm{p}_{\text {rel }} & =\sqrt{\frac{\left(\mathbf{p}_{\mathbf{1}}-\mathbf{p}_{\mathbf{2}}\right)^{2}}{4}}=\sqrt{\frac{\left(2 \mathbf{p}_{\mathbf{1}}-\mathbf{p}_{\mathrm{NN}}\right)^{2}}{4}} \\
& =\sqrt{\mathrm{p}_{1}^{2}+\frac{\mathrm{p}_{\mathrm{NN}}^{2}}{4}-\mathrm{p}_{1} \mathrm{p}_{\mathrm{NN}} \cos \tilde{\theta}_{1 N N}} .
\end{aligned}
$$

Also

$$
\begin{aligned}
\omega_{\text {rel }} & =\frac{M_{N}-E_{m}-T_{A-1}}{\frac{M_{A}}{A}} \\
& =\frac{A}{M_{A}}\left(M_{N}-E_{m}-\frac{\mathrm{p}_{1}^{2}}{2 M_{A-1}}\right),
\end{aligned}
$$

and the cosine of the angle $\theta_{1 \text { rel }}$, between $\mathbf{p}_{1}$ and $\mathbf{p}_{\text {rel }}$, is given by

$$
\cos \theta_{1 \mathrm{rel}}=\frac{2 \mathrm{p}_{1}-\mathrm{p}_{\mathrm{NN}} \cos \tilde{\theta}_{1 \mathrm{NN}}}{2 \mathrm{p}_{\mathrm{rel}}}
$$


with the restriction

$$
\cos ^{2} \theta_{1 \text { rel }} \leq 1
$$

The momentum of the correlated nucleon 2 is

$$
\mathrm{p}_{2}=\sqrt{\left(\mathbf{p}_{\mathbf{1}}-\mathbf{2} \mathbf{p}_{\text {rel }}\right)^{2}}=\sqrt{\mathrm{p}_{1}^{2}+4 \mathrm{p}_{\text {rel }}^{2}-4 \mathrm{p}_{1} \mathrm{p}_{\text {rel }} \cos \theta_{1 \text { rel }}}
$$

with the restriction

$$
\mathrm{p}_{2}>\mathrm{k}_{\mathrm{F}} \sim 0.250 \mathrm{GeV} / \mathrm{c} \text {. }
$$

The 2N SRC nuclear spectral functions [Eq.(3.13)], together with Eqs. (2.36), (3.8) to (3.10), and (3.14) to (3.19), and the normalization condition [Eq.(2.35)], constitute the computational model from which numerical estimates are obtained in the chapter 4 of the present dissertation.

\subsubsection{Computational model for three nucleon SRC nuclear spectral function in virtual nucleon approximation}

The following expression for the 3N SRC nuclear spectral function in VN approximation is obtained by inserting the product of the relative momentum distributions of the NN SRC [Eq.(2.49)] in Eq. (2.47), namely

$S_{A, 3 N}^{p / n}\left(\mathrm{p}_{1}, E_{m}\right)=a_{2}^{2}(A, Z) C^{p / n}(A, N) \int \frac{n_{d}\left(\mathrm{p}_{3}\right) n_{d}\left(\mathrm{p}_{12}\right)}{\frac{M_{N}-E_{m}-T_{A-1}}{M_{A} / A}} \Theta\left(\mathrm{p}_{3}-k_{s r c}\right) \Theta\left(\mathrm{p}_{12}-k_{s r c}\right) \delta\left(E_{m}-E_{m}^{3 N}\right) d^{3} \mathbf{p}_{3}$,

where $p / n$ indicates proton and neutron respectively, and the $C^{p / n}(A, N)$ factors are given by Eq. (3.3). 
The relativistic kinetic energies of the on-shell correlated nucleons 2 and 3 [Fig. 2.3], aregiven by

$$
\begin{gathered}
T_{2}=\sqrt{\mathrm{p}_{2}^{2}+M_{N}^{2}}-M_{N}=\sqrt{\left(\mathbf{p}_{1}+\mathbf{p}_{3}\right)^{2}+M_{N}^{2}}-M_{N} \\
=\sqrt{\mathrm{p}_{1}^{2}+2 \mathrm{p}_{1} \mathrm{p}_{3} \cos \theta_{13}+\mathrm{p}_{3}^{2}+M_{N}^{2}}-M_{N}, \\
T_{3}=\sqrt{\mathrm{p}_{3}^{2}+M_{N}^{2}}-M_{N},
\end{gathered}
$$

where $\theta_{13}$ is the angle between $\mathbf{p}_{1}$ and $\mathbf{p}_{3}$. Then, the removal energy of the bound nucleon $E_{m}^{3 N}$ [Eq. (2.38)] can be expressed as

$$
E_{m}^{3 N}=E_{t h r}^{(3)}-2 M_{N}-\frac{\mathrm{p}_{1}^{2}}{2 M_{A-1}}+\sqrt{\mathrm{p}_{3}^{2}+M_{N}^{2}}+\sqrt{\mathrm{p}_{1}^{2}+2 \mathrm{p}_{1} \mathrm{p}_{3} \cos \theta_{13}+\mathrm{p}_{3}^{2}+M_{N}^{2}}
$$

The argument of the delta function in the nuclear spectral function [Eq.(3.20)] is therefore

$$
E_{m}-E_{m}^{3 N}=E_{m}-E_{t h r}^{(3)}+2 M_{N}+\frac{\mathrm{p}_{1}^{2}}{2 M_{A-1}}-\sqrt{\mathrm{p}_{3}^{2}+M_{N}^{2}}-\sqrt{\mathrm{p}_{1}^{2}+2 \mathrm{p}_{1} \mathrm{p}_{3} \cos \theta_{13}+\mathrm{p}_{3}^{2}+M_{N}^{2}}
$$

where $E_{t h r}^{(3)} \approx 3 M_{N}+M_{A-3}-M_{A}$.

Similar to the case of the 2N SRC nuclear spectral function in section (3.1.1), the integration of the delta function in the spectral functions [Eq.(3.20)] over $\cos \theta_{13}$ yields

$$
\int \delta^{0}\left[E_{m}-E_{m}^{3 N}\right] d \mathbf{p}_{3}=2 \pi \int \delta^{0}\left[E_{m}-E_{m}^{3 N}\right] \mathrm{p}_{3}^{2} d \mathrm{p}_{3} \cos \theta_{13}=2 \pi \int \frac{E_{30}}{\mathrm{p}_{1}} \mathrm{p}_{3} d \mathrm{p}_{3},
$$

where $E_{30}$ is the relativistic energy of the nucleon 3 for $\cos \tilde{\theta}_{13}$, given by

$$
E_{30}=\sqrt{\mathrm{p}_{1}^{2}+2 \mathrm{p}_{1} \mathrm{p}_{3} \cos \tilde{\theta}_{13}+\mathrm{p}_{3}^{2}+M_{N}^{2}}=E_{m}-E_{t h r}^{(3)}+2 M_{N}+\frac{\mathrm{p}_{1}^{2}}{2 M_{A-1}}-\sqrt{\mathrm{p}_{3}^{2}+M_{N}^{2}},
$$

and $\cos \tilde{\theta}_{13}$, the root of $E_{m}-E_{m}^{3 N}=0$, is

$$
\cos \tilde{\theta}_{13}=\frac{E_{30}^{2}-\mathrm{p}_{1}^{2}-\mathrm{p}_{3}^{2}-M_{N}^{2}}{2 \mathrm{p}_{1} \mathrm{p}_{3}}
$$


with the restrictions

$$
\begin{aligned}
E_{30}^{2} & \geq 0, \\
\cos ^{2} \tilde{\theta}_{13} & \leq 1 .
\end{aligned}
$$

The 3N SRC nuclear spectral functions [Eq.(3.20)] are therefore given by:

$$
S_{A, 3 N}^{p / n}\left(\mathrm{p}_{1}, E_{m}\right)=2 \pi a_{2}^{2}(A, Z) C^{p / n}(A, N) \int_{0}^{1} \frac{n_{d}\left(\mathrm{p}_{3}\right)}{\omega_{3 N}} n_{d}\left(\mathrm{p}_{12}\right) \frac{E_{30}}{\mathrm{p}_{1}} \Theta\left(\mathrm{p}_{3}-k_{s r c}\right) \Theta\left(\mathrm{p}_{12}-k_{s r c}\right) \mathrm{p}_{3} d \mathrm{p}_{3},
$$

where, by using the collinear assumption within the $\mathrm{VN}$ approximation $\left[\mathbf{p}_{\mathbf{1}}+\mathbf{p}_{\mathbf{2}}+\mathbf{p}_{\mathbf{3}}=0\right]$,

$$
\begin{aligned}
\mathrm{p}_{12} & =\sqrt{\frac{\left(\mathbf{p}_{\mathbf{1}}-\mathbf{p}_{\mathbf{2}}\right)^{2}}{4}}=\sqrt{\frac{\left(2 \mathbf{p}_{\mathbf{1}}+\mathbf{p}_{\mathbf{3}}\right)^{2}}{4}} \\
& =\sqrt{\mathrm{p}_{1}^{2}+\frac{\mathrm{p}_{3}^{2}}{4}+\mathrm{p}_{1} \mathrm{p}_{3} \cos \tilde{\theta}_{13}}
\end{aligned}
$$

and

$$
\begin{aligned}
\omega_{3 \mathrm{~N}} & =\frac{M_{N}-E_{m}-T_{A-1}}{\frac{M_{A}}{A}} \\
& =\frac{A}{M_{A}}\left(M_{N}-E_{m}-\frac{\mathrm{p}_{1}^{2}}{2 M_{A-1}}\right) .
\end{aligned}
$$

The cosine of the angle $\theta_{112}$, between $\mathbf{p}_{1}$ and $\mathbf{p}_{\mathbf{1 2}}$, is given by

$$
\cos \theta_{112}=\frac{2 \mathrm{p}_{1}+\mathrm{p}_{3} \cos \tilde{\theta}_{13}}{2 \mathrm{p}_{12}}
$$

with the restriction

$$
\cos ^{2} \theta_{112} \leq 1
$$


The momentum of the correlated nucleon 2 is

$$
\mathrm{p}_{2}=\sqrt{\left(\mathbf{p}_{\mathbf{1}}-\mathbf{2} \mathbf{p}_{\mathbf{1 2}}\right)^{2}}=\sqrt{\mathrm{p}_{1}^{2}+4 \mathrm{p}_{12}^{2}-4 \mathrm{p}_{1} \mathrm{p}_{12} \cos \theta_{112}},
$$

with the restriction

$$
\mathrm{p}_{2}>\mathrm{k}_{\mathrm{F}} \sim 0.250 \mathrm{GeV} / \mathrm{c}
$$

The 3N SRC nuclear spectral functions [Eq.(3.29)], together with Eqs. (3.26) to (3.28), and (3.30) to (3.35), and the normalization condition [Eq.(2.48)], constitute the computational model from which numerical estimates are obtained in the chapter 4 of the present dissertation.

\subsection{Computational models for SRC nuclear spectral function in light-front approximation}

The computational models for high momentum nuclear spectral function in light-front (LF) approximation, with two and three nucleons in short range correlation, are developed in the present section.

\subsubsection{Computational model for two nucleon SRC nuclear spectral function in light-front approximation}

The following expression for the 2N SRC nuclear spectral function in LF approximation is obtained by inserting the light-front density matrix for the relative motion in the 2N SRC [Eq.(2.99)] in Eq. (2.97), namely

$$
\begin{array}{r}
P_{A, 2 N}^{p / n}\left(\alpha_{1}, \mathrm{p}_{1, \perp}, \tilde{M}_{N}^{2}\right)=\frac{a_{2}(A, Z)}{\left(2 x_{p / n}\right)^{\gamma}} M_{N} \int \rho_{d}\left(\beta_{1}, \mathrm{k}_{1, \perp}\right) \Theta\left(\mathrm{k}_{1}-\mathrm{k}_{s r c}\right) n_{C M}\left(\mathrm{k}_{C M}\right) 2 \delta\left(\alpha_{1}+\alpha_{2}-\alpha_{N N}\right) \\
\delta^{2}\left(\mathbf{p}_{1, \perp}+\mathbf{p}_{2, \perp}-\mathbf{p}_{N N, \perp}\right) \delta\left(\tilde{M}_{N}^{2}-\tilde{M}_{N}^{(2 N), 2}\right) d \beta_{2} d^{2} \mathbf{p}_{2, \perp} d \alpha_{N N} d^{2} \mathbf{p}_{N N, \perp},
\end{array}
$$


where $p / n$ indicates proton and neutron respectively, $\beta_{1}$ is defined in Eq. (2.78), $x_{p / n}$ are given by Eq. (3.2), and the light-front density matrix for the deuteron is defined as

$$
\rho_{d}\left(\beta_{1}, \mathrm{k}_{1, \perp}\right)=\frac{n_{d}\left(\mathrm{k}_{1}\right)}{2-\beta_{1}} \sqrt{M_{N}^{2}+\mathrm{k}_{1}}=\frac{n_{d}\left(\mathrm{k}_{1}\right)}{2-\beta_{1}} \sqrt{\frac{s_{N N}}{4}} \Theta\left(\mathrm{k}_{1}-\mathrm{k}_{\mathrm{src}}\right)
$$

where the invariant energy $s_{N N}$ and the relative momentum $\mathbf{k}_{1}$ are defined in Eq. (2.80) and the parameter $a_{2}(A, Z)$ in Eq. (2.37). $n_{d}$ is the deuteron momentum distribution calculated from a nonrelativistic wave function. The normalization of this density matrix is given by

$$
\int \rho_{d}\left(\beta_{1}, \mathrm{k}_{1, \perp}\right) \frac{d \beta_{1}}{\beta_{1}} d^{2} \mathbf{k}_{1}=1
$$

Integration in the nuclear spectral function [Eq. (3.36)] by $d^{2} \mathbf{p}_{2, \perp}$ through $\delta^{2}\left(\mathbf{p}_{1, \perp}+\mathbf{p}_{2, \perp}-\right.$ $\left.\mathbf{p}_{N N, \perp}\right)$, and by $d \beta_{2}$ through $\delta\left(\alpha_{1}+\alpha_{2}-\alpha_{N N}\right)=2 \delta\left(\beta_{1}+\beta_{2}-2\right) / \alpha_{N N}$, and considering that $d^{2} \mathbf{p}_{N N, \perp}=\mathrm{p}_{N N, \perp} d \mathrm{p}_{N N, \perp} d \phi_{N N}$ yields

$$
\begin{gathered}
P_{A, 2 N}^{p / n}\left(\alpha_{1}, \mathrm{p}_{1, \perp}, \tilde{M}_{N}^{2}\right)=\frac{a_{2}(A, Z)}{\left(2 x_{p / n}\right)^{\gamma}} M_{N} \int \rho_{d}\left(\beta_{1}, \mathrm{k}_{1, \perp}\right) \Theta\left(\mathrm{k}_{1}-\mathrm{k}_{s r c}\right) n_{C M}\left(\mathrm{k}_{C M}\right) \\
\delta\left(\tilde{M}_{N}^{2}-\tilde{M}_{N}^{(2 N), 2}\right) \frac{4 d \alpha_{N N}}{\alpha_{N N}} \mathrm{p}_{N N, \perp} d \mathrm{p}_{N N, \perp} d \phi_{N N}
\end{gathered}
$$

where $\phi_{N N}$ is the angle between $\mathbf{p}_{1, \perp}$ and $\mathbf{p}_{N N, \perp}$, and

$$
\beta_{1}+\beta_{2}=2
$$

$$
\mathbf{p}_{1, \perp}+\mathbf{p}_{2, \perp}=\mathbf{p}_{N N, \perp}
$$


From Eqs. (3.40) and (3.41), the invariant mass $\tilde{M}_{N}^{(2 N), 2}$ [Eq.(2.66)] can be expressed as

$$
\begin{aligned}
\tilde{M}_{N}^{(2 N), 2} & =p_{1}^{-} p_{1}^{+}-\mathrm{p}_{1, \perp}^{2}=p_{1}^{+}\left(p_{N N}^{-}-p_{2}^{-}\right)-\mathrm{p}_{1, \perp}^{2} \\
& =\alpha_{1}\left(\frac{M_{N N}^{2}+\mathrm{p}_{N N, \perp}^{2}}{\alpha_{N N}}-\frac{M_{N}^{2}+\mathrm{p}_{2, \perp}^{2}}{\alpha_{2}}\right)-\mathrm{p}_{1, \perp}^{2} \\
& =\frac{2-\beta_{2}}{2}\left\{M_{N N}^{2}+\mathrm{p}_{N N, \perp}^{2}-\frac{2}{\beta_{2}}\left[M_{N}^{2}+\left(\mathbf{p}_{N N, \perp}-\mathbf{p}_{1, \perp}^{2}\right)^{2}\right]\right\}-\mathrm{p}_{1, \perp}^{2} \\
& =\frac{\beta_{1}^{2}}{2\left(2-\beta_{1}\right)}\left(\frac{2-\beta_{1}}{\beta_{1}} M_{N N}^{2}-\frac{2}{\beta_{1}} M_{N}^{2}-\mathrm{p}_{N N, \perp}^{2}+\frac{4}{\beta_{1}} \mathrm{p}_{N N, \perp} \mathrm{p}_{1, \perp} \cos \phi_{N N}-\frac{4}{\beta_{1}^{2}} \mathrm{p}_{1, \perp}^{2}\right),
\end{aligned}
$$

where $M_{N N}$ is taken as the deuteron mass.

Inserting Eq. (3.42) in the argument of the delta function in the nuclear spectral function [Eq.(3.39)] yields

$$
\tilde{M}_{N}^{2}-\tilde{M}_{N}^{(2 N), 2}=\frac{\beta_{1}^{2}}{2\left(2-\beta_{1}\right)}\left(\mathrm{p}_{N N, \perp}^{2}-\frac{4}{\beta_{1}} \mathrm{p}_{1, \perp} \mathrm{p}_{N N, \perp} \cos \phi_{N N}+\mathrm{p}_{02, \perp}^{2}\right)
$$

where

$$
\mathrm{p}_{02, \perp}^{2}=\frac{2}{\beta_{1}}\left(\frac{2}{\beta_{1}} \mathrm{p}_{1, \perp}^{2}+\frac{2-\beta_{1}}{\beta_{1}} \tilde{M}_{N}^{2}-\frac{2-\beta_{1}}{2} M_{N N}^{2}+M_{N}^{2}\right)
$$

with the restriction

$$
\mathrm{p}_{02, \perp}^{2} \geq 0
$$

The roots of $\tilde{M}_{N}^{2}-\tilde{M}_{N}^{(2 N), 2}=0$ are

$$
\begin{aligned}
& \tilde{\mathrm{p}}_{N N, \perp}^{a}=\frac{2}{\beta_{1}}\left(\mathrm{p}_{1, \perp} \cos \phi_{N N}+\frac{1}{4} \sqrt{16 \mathrm{p}_{1, \perp}^{2} \cos ^{2} \phi_{N N}-\beta_{1}^{2} \mathrm{p}_{02, \perp}^{2}}\right), \\
& \tilde{\mathrm{p}}_{N N, \perp}^{b}=\frac{2}{\beta_{1}}\left(\mathrm{p}_{1, \perp} \cos \phi_{N N}-\frac{1}{4} \sqrt{16 \mathrm{p}_{1, \perp}^{2} \cos ^{2} \phi_{N N}-\beta_{1}^{2} \mathrm{p}_{02, \perp}^{2}}\right),
\end{aligned}
$$

with the restriction

$$
16 \mathrm{p}_{1, \perp}^{2} \cos ^{2} \phi_{N N}-\beta_{1}^{2} \mathrm{p}_{02, \perp}^{2}>0 .
$$


Then, from Eqs. (3.1) and (3.46), integration of the delta function in the nuclear spectral function [Eq.(3.39)] over $d \mathrm{p}_{N N, \perp}$, yields

$$
\begin{aligned}
P_{A, 2 N}^{p / n}\left(\alpha_{1}, \mathrm{p}_{1, \perp}, \tilde{M}_{N}^{2}\right) & =\frac{a_{2}(A, Z)}{\left(2 x_{p / n}\right)^{\gamma}} M_{N} \int_{\alpha_{1}}^{A} \int_{0}^{2 \pi}\left[\rho_{d}\left(\beta_{1}, \mathrm{k}_{1, \perp}^{a}\right) n_{C M}\left(\mathrm{k}_{C M}^{a}\right) \tilde{\mathrm{p}}_{N N, \perp}^{a} \Theta\left(\mathrm{k}_{1}^{a}-\mathrm{k}_{s r c}\right)\right. \\
& \left.+\rho_{d}\left(\beta_{1}, \mathrm{k}_{1, \perp}^{b}\right) n_{C M}\left(\mathrm{k}_{C M}^{b}\right) \tilde{\mathrm{p}}_{N N, \perp}^{b} \Theta\left(\mathrm{k}_{1}^{b}-\mathrm{k}_{s r c}\right)\right] \\
& \left(\frac{2-\beta_{1}}{\beta_{1}}\right) \frac{2}{\sqrt{16 \mathrm{p}_{1, \perp}^{2} \cos ^{2} \phi_{N N}-\beta_{1}^{2} \mathrm{p}_{02, \perp}^{2}}} \frac{4 d \alpha_{N N}}{\alpha_{N N}} d \phi_{N N}
\end{aligned}
$$

where from Eqs. (2.78) and (2.80) it follows that

$$
\begin{gathered}
\mathrm{k}_{1, \perp}^{a(b)}=\sqrt{\mathrm{p}_{1, \perp}^{2}+\left(\frac{\beta_{1}}{2} \tilde{\mathrm{p}}_{N N, \perp}^{a(b)}\right)^{2}-\beta_{1} \mathrm{p}_{1, \perp} \tilde{\mathrm{p}}_{N N, \perp}^{a(b)} \cos \phi_{N N},} \\
s_{N N}^{a(b)}=\frac{4}{\beta_{1}\left(2-\beta_{1}\right)}\left[M_{N}^{2}+\left(\mathrm{k}_{1, \perp}^{a(b)}\right)^{2}\right] \\
\mathrm{k}_{1}^{a(b)}=\frac{1}{2} \sqrt{s_{N N}^{a(b)}-4 M_{N}^{2}},
\end{gathered}
$$

and from Eqs. (2.86) and (2.89) with $\mathbf{p}_{A, \perp}=0$

$$
\begin{gathered}
s_{N N, A-2}^{a(b)}=A^{2} \frac{\left[M_{N N}^{2}+\frac{\alpha_{N N}}{A}\left(M_{A-2}^{2}-M_{N N}^{2}\right)+\left(\tilde{\mathrm{p}}_{N N, \perp}^{a(b)}\right)^{2}\right]}{\alpha_{N N}\left(A-\alpha_{N N}\right)}, \\
k_{C M}^{a(b)}=\frac{\sqrt{\left[s_{N N, A-2}^{a(b)}-\left(M_{N N}+M_{A-2}\right)^{2}\right]\left[s_{N N, A-2}^{a(b)}-\left(M_{N N}-M_{A-2}\right)^{2}\right]}}{2 \sqrt{s_{N N, A-2}^{a(b)}}} .
\end{gathered}
$$


Following a similar calculation as above, integration of the delta function in the nuclear spectral function [Eq.(3.39)] by $d \phi_{N N}$ yields

$$
\begin{aligned}
P_{A, 2 N}^{p / n}\left(\alpha_{1}, \mathrm{p}_{1, \perp}, \tilde{M}_{N}^{2}\right) & =\frac{a_{2}(A, Z)}{\left(2 x_{p / n}\right)^{\gamma}} M_{N} \int_{\alpha_{1}}^{A} \int_{0}^{1} \rho_{d}\left(\beta_{1}, \mathrm{k}_{1, \perp}\right) \Theta\left(\mathrm{k}_{1}-\mathrm{k}_{s r c}\right) n_{C M}\left(\mathrm{k}_{C M}\right) \\
& \left(\frac{2-\beta_{1}}{\beta_{1}}\right) \frac{2}{\left.\sqrt{16 \mathrm{p}_{1, \perp}^{2} \mathrm{p}_{N N, \perp}^{2}-\beta_{1}^{2}\left(\mathrm{p}_{N N, \perp}^{2}+\mathrm{p}_{02, \perp}^{2}\right.}\right)^{2}} \frac{4 d \alpha_{N N}}{\alpha_{N N}} \mathrm{p}_{N N, \perp} d \mathrm{p}_{N N, \perp}
\end{aligned}
$$

with the restriction

$$
16 \mathrm{p}_{1, \perp}^{2} \mathrm{p}_{N N, \perp}^{2}-\beta_{1}^{2}\left(\mathrm{p}_{N N, \perp}^{2}+\mathrm{p}_{02, \perp}^{2}\right)^{2}>0
$$

and

$$
\cos \tilde{\phi}_{N N}=\left(\frac{\beta_{1}}{4}\right) \frac{\mathrm{p}_{N N, \perp}^{2}+\mathrm{p}_{02, \perp}^{2}}{\mathrm{p}_{1, \perp} \mathrm{p}_{N N, \perp}}
$$

is the root of $\tilde{M}_{N}^{2}-\tilde{M}_{N}^{(2 N), 2}=0$ for $\cos \phi_{N N}$, with the restriction

$$
\cos ^{2} \tilde{\phi}_{N N} \leq 1
$$

From Eq. (2.78) it follows that

$$
\mathrm{k}_{1, \perp}=\sqrt{\mathrm{p}_{1, \perp}^{2}+\left(\frac{\beta_{1}}{2} \mathrm{p}_{N N, \perp}\right)^{2}-\beta_{1} \mathrm{p}_{1, \perp} \mathrm{p}_{N N, \perp} \cos \tilde{\phi}_{N N}}
$$

The momenta $k_{1}$ and $k_{C M}$ are calculated from Eqs. (2.80), and (2.86) and (2.89) respectively, with $\mathbf{p}_{A, \perp}=0$.

The 2N SRC nuclear spectral functions [Eq.(3.54)], together with Eqs.(2.36), (2.80), (2.86), (2.89), (3.44), and (3.55) to (3.58), and the normalization conditions [Eq.(2.98)], [Eq.(2.100)] and [Eq.(2.101)], constitute the simplest computational model from which numerical estimates are obtained in the chapter 4 of the present dissertation. The Eqs. (2.36), (3.44) to (3.53), (2.98), (2.100) and (2.101), also constitute a computational model but more calculations are needed to obtain the same numerical estimates. 


\subsubsection{Computational model for three nucleon SRC nuclear spectral function in light-front approximation}

The following expression for the 3N SRC nuclear spectral function in LF approximation is obtained by inserting the product of light-front density matrices for the relative motion in the $2 \mathrm{~N}$ SRC [Eq.(2.133)] in Eq. (2.131), namely

$$
\begin{aligned}
P_{A, 3 N}^{p / n}\left(\alpha_{1}, \mathrm{p}_{1, \perp}, \tilde{M}_{N}\right) & =a_{2}^{2}(A, Z) C^{p / n}(A, Z) \int \frac{3-\alpha_{3}}{2\left(2-\alpha_{3}\right)^{2}} \rho_{d}\left(\beta_{2^{\prime}}, \mathrm{p}_{3, \perp}\right) \Theta\left(\tilde{\mathrm{k}}_{1}-\mathrm{k}_{s r c}\right) \rho_{d}\left(\beta_{1}, \tilde{\mathrm{k}}_{1, \perp}\right) \Theta\left(\tilde{\mathrm{k}}_{3}-\mathrm{k}_{s r c}\right) \\
2 & \delta\left(\alpha_{1}+\alpha_{2}+\alpha_{3}-3\right) \delta^{2}\left(\mathbf{p}_{1, \perp}+\mathbf{p}_{2, \perp}+\mathbf{p}_{3, \perp}\right) \delta\left(\tilde{M}_{N}^{2}-M_{N}^{(3 N), 2}\right) d \alpha_{2} d^{2} \mathbf{p}_{2, \perp} d \alpha_{3} d^{2} \mathbf{p}_{3, \perp},
\end{aligned}
$$

where $p / n$ indicates proton and neutron respectively, $\beta_{2^{\prime}}$ and $\beta_{1}$ are given by Eq. (2.115), the light-front density matrix for the deuteron by Eq. (3.37), and the factors $C^{p / n}(A, Z)$ by Eq. (3.3).

Integration in the nuclear spectral function [Eq. (3.59)] by $d^{2} \mathbf{p}_{2, \perp}$ through $\delta^{2}\left(\mathbf{p}_{1, \perp}+\mathbf{p}_{2, \perp}+\right.$ $\left.\mathbf{p}_{3, \perp}\right)$, and by $d \alpha_{2}$ through $\delta\left(\alpha_{1}+\alpha_{2}+\alpha_{3}-3\right)$, and considering that $d^{2} \mathbf{p}_{3, \perp}=\mathrm{p}_{3, \perp} d \mathrm{p}_{3, \perp} d \phi_{13}$ yields

$$
\begin{aligned}
P_{A, 3 N}^{p / n}\left(\alpha_{1}, \mathrm{p}_{1, \perp}, \tilde{M}_{N}\right) & =a_{2}^{2}(A, Z) C^{p / n}(A, Z) \int \frac{3-\alpha_{3}}{\left(2-\alpha_{3}\right)^{2}} \rho_{d}\left(\beta_{2^{\prime}}, \mathrm{p}_{3, \perp}\right) \Theta\left(\tilde{\mathrm{k}}_{1}-\mathrm{k}_{s r c}\right) \rho_{d}\left(\beta_{1}, \tilde{\mathrm{k}}_{1, \perp}\right) \Theta\left(\tilde{\mathrm{k}}_{3}-\mathrm{k}_{s r c}\right) \\
& \delta\left(\tilde{M}_{N}^{2}-M_{N}^{(3 N), 2}\right) d \alpha_{3} \mathrm{p}_{3, \perp} d \mathrm{p}_{3, \perp} d \phi_{13}
\end{aligned}
$$

where $\phi_{13}$ is the angle between $\mathbf{p}_{1, \perp}$ and $\mathbf{p}_{3, \perp}$, and

$$
\begin{gathered}
\alpha_{2}=3-\alpha_{1}-\alpha_{3}, \\
\mathbf{p}_{2, \perp}=-\mathbf{p}_{1, \perp}-\mathbf{p}_{3, \perp} .
\end{gathered}
$$


From Eqs. (3.61) and (3.62), the invariant mass $\tilde{M}_{N}^{(3 N), 2}[$ Eq.(2.104)] can be expressed as

$$
\begin{aligned}
\tilde{M}_{N}^{(3 N), 2} & =\frac{\alpha_{1}}{3}\left(M_{3 N}^{2}-\frac{M_{N}^{2}+\mathrm{p}_{2, \perp}^{2}}{\alpha_{2} / 3}-\frac{M_{N}^{2}+\mathrm{p}_{3, \perp}^{2}}{\alpha_{3} / 3}\right)-\mathrm{p}_{1, \perp}^{2} \\
& =\frac{\alpha_{1}}{3}\left[M_{3 N}^{2}-\frac{M_{N}^{2}+\left(\mathbf{p}_{1, \perp}+\mathbf{p}_{3, \perp}\right)^{2}}{\left(3-\alpha_{1}-\alpha_{3}\right) / 3}-\frac{M_{N}^{2}+\mathrm{p}_{3, \perp}^{2}}{\alpha_{3} / 3}\right]-\mathrm{p}_{1, \perp}^{2} \\
& =\frac{\alpha_{1}\left(3-\alpha_{1}\right)}{\alpha_{3}\left(3-\alpha_{1}-\alpha_{3}\right)} \\
& \times\left[\frac{\alpha_{3}\left(3-\alpha_{1}-\alpha_{3}\right)}{3\left(3-\alpha_{1}\right)} M_{3 N}^{2}-M_{N}^{2}-\frac{\alpha_{3}\left(3-\alpha_{3}\right)}{\alpha_{1}\left(3-\alpha_{1}\right)} \mathrm{p}_{1, \perp}^{2}-\mathrm{p}_{3, \perp}^{2}+\frac{\alpha_{3}}{\alpha_{1}-3} \mathrm{p}_{1, \perp} \cos \phi_{13} \mathrm{p}_{3, \perp}\right]
\end{aligned}
$$

where $M_{3 N}^{2}$ is given by Eq. (2.105).

Inserting Eq. (3.63) in the argument of the delta function in the nuclear spectral function [Eq.(3.60)] yields

$$
\tilde{M}_{N}^{2}-\tilde{M}_{N}^{(3 N), 2}=\frac{\alpha_{1}\left(3-\alpha_{1}\right)}{\alpha_{3}\left(3-\alpha_{1}-\alpha_{3}\right)}\left(\mathrm{p}_{3, \perp}^{2}-\frac{\alpha_{3}}{\alpha_{1}-3} \mathrm{p}_{1, \perp} \cos \phi_{13} \mathrm{p}_{3, \perp}-\mathrm{p}_{03, \perp}^{2}\right)
$$

where

$$
\mathrm{p}_{03, \perp}^{2}=\frac{\alpha_{3}\left(3-\alpha_{1}-\alpha_{3}\right)}{\alpha_{1}\left(\alpha_{1}-3\right)}\left[\tilde{M}_{N}^{2}-\frac{\alpha_{1}}{3} M_{3 N}^{2}+\frac{\alpha_{1}\left(3-\alpha_{1}\right)}{\alpha_{3}\left(3-\alpha_{1}-\alpha_{3}\right)} M_{N}^{2}+\frac{3-\alpha_{3}}{3-\alpha_{1}-\alpha_{3}} \mathrm{p}_{1, \perp}^{2}\right]
$$

with the restriction

$$
\mathrm{p}_{03, \perp}^{2} \geq 0
$$

Similar to the case of $2 \mathrm{~N}$ SRC in section (3.2.1), integration of the delta function in the nuclear spectral function [Eq.(3.60)] by $d \mathrm{p}_{3, \perp}$ yields

$$
\begin{aligned}
P_{A, 3 N}^{p / n}\left(\alpha_{1}, \mathrm{p}_{1, \perp}, \tilde{M}_{N}\right) & =a_{2}^{2}(A, Z) C^{p / n}(A, Z) \int_{0}^{3-\alpha_{1}} \int_{0}^{2 \pi} \frac{3-\alpha_{3}}{\left(2-\alpha_{3}\right)^{2}} \\
& {\left[\rho_{d}\left(\beta_{2^{\prime}}, \tilde{\mathrm{p}}_{3, \perp}^{a}\right) \Theta\left(\tilde{\mathrm{k}}_{1}^{a}-\mathrm{k}_{s r c}\right) \rho_{d}\left(\beta_{1}, \tilde{\mathrm{k}}_{1, \perp}^{a}\right) \Theta\left(\tilde{\mathrm{k}}_{3}^{a}-\mathrm{k}_{s r c}\right) \tilde{\mathrm{p}}_{3, \perp}^{a}\right.} \\
& \left.+\rho_{d}\left(\beta_{2^{\prime}}, \tilde{\mathrm{p}}_{3, \perp}^{b}\right) \Theta\left(\tilde{\mathrm{k}}_{1}^{b}-\mathrm{k}_{s r c}\right) \rho_{d}\left(\beta_{1}, \tilde{\mathrm{k}}_{1, \perp}^{b}\right) \Theta\left(\tilde{\mathrm{k}}_{3}^{b}-\mathrm{k}_{s r c}\right) \tilde{\mathrm{p}}_{3, \perp}^{b}\right] \\
& \frac{\alpha_{3}\left(3-\alpha_{1}-\alpha_{3}\right)\left|\alpha_{1}-3\right|}{\alpha_{1}\left(3-\alpha_{1}\right)} \frac{1}{\sqrt{\alpha_{3}^{2} \mathrm{p}_{1, \perp}^{2} \cos ^{2} \phi_{13}+4\left(\alpha_{1}-3\right)^{2} \mathrm{p}_{03, \perp}^{2}}} d \alpha_{3} d \phi_{13},
\end{aligned}
$$


with the restriction

$$
\alpha_{3}^{2} \mathrm{p}_{1, \perp}^{2} \cos ^{2} \phi_{13}+4\left(\alpha_{1}-3\right)^{2} \mathrm{p}_{03, \perp}^{2}>0,
$$

and

$$
\begin{aligned}
& \tilde{\mathrm{p}}_{3, \perp}^{a}=\frac{\alpha_{3}}{2\left(\alpha_{1}-3\right)} \mathrm{p}_{1, \perp} \cos \phi_{13}+\frac{1}{2\left|\alpha_{1}-3\right|} \sqrt{\alpha_{3}^{2} \mathrm{p}_{1, \perp}^{2} \cos ^{2} \phi_{13}+4\left(\alpha_{1}-3\right)^{2} \mathrm{p}_{03, \perp}^{2}}, \\
& \tilde{\mathrm{p}}_{3, \perp}^{b}=\frac{\alpha_{3}}{2\left(\alpha_{1}-3\right)} \mathrm{p}_{1, \perp} \cos \phi_{13}-\frac{1}{2\left|\alpha_{1}-3\right|} \sqrt{\alpha_{3}^{2} \mathrm{p}_{1, \perp}^{2} \cos ^{2} \phi_{13}+4\left(\alpha_{1}-3\right)^{2} \mathrm{p}_{03, \perp}^{2}},
\end{aligned}
$$

are the roots of $\tilde{M}_{N}^{2}-\tilde{M}_{N}^{(3 N), 2}=0$.

From Eqs. (2.125), (2.126), and (2.118) it follows that

$$
\begin{gathered}
\tilde{\mathrm{k}}_{1}^{a(b)}=\frac{M_{N}^{2}+\left(\tilde{\mathrm{k}}_{1, \perp}^{a(b)}\right)^{2}}{\beta_{1}\left(2-\beta_{1}\right)}-M_{N}^{2}, \\
\tilde{\mathrm{k}}_{1, \perp}^{a(b)}=\sqrt{\mathrm{p}_{1, \perp}^{2}+\left(\frac{\beta_{1}}{2} \tilde{\mathrm{p}}_{3, \perp}^{a(b)}\right)^{2}+\beta_{1} \mathrm{p}_{1, \perp} \tilde{\mathrm{p}}_{3, \perp}^{a(b)} \cos \phi_{13},} \\
\tilde{\mathrm{k}}_{3}^{a(b)}=\frac{M_{N}^{2}+\left(\tilde{\mathrm{p}}_{3, \perp}^{a(b)}\right)^{2}}{\beta_{3}\left(2-\beta_{3}\right)}-M_{N}^{2} .
\end{gathered}
$$

Following a similar calculation as above, integration of the delta function in Eq.(3.60) by $d \phi_{13}$ yields

$$
\begin{aligned}
P_{A, 3 N}^{p / n}\left(\alpha_{1}, \mathrm{p}_{1, \perp}, \tilde{M}_{N}\right) & =a_{2}^{2}(A, Z) C^{p / n}(A, Z) \int_{3-\alpha_{1}}^{3} \int_{0}^{1} \frac{3-\alpha_{3}}{\left(2-\alpha_{3}\right)^{2}} \rho_{d}\left(\beta_{2^{\prime}}, \mathrm{p}_{3, \perp}\right) \Theta\left(\tilde{\mathrm{k}}_{1}-\mathrm{k}_{s r c}\right) \rho_{d}\left(\beta_{1}, \tilde{\mathrm{k}}_{1, \perp}\right) \\
& \Theta\left(\tilde{\mathrm{k}}_{3}-\mathrm{k}_{s r c}\right) \frac{\alpha_{3}\left(3-\alpha_{1}-\alpha_{3}\right)\left|\alpha_{1}-3\right|}{\alpha_{1}\left(3-\alpha_{1}\right)} \\
& \frac{1}{\sqrt{\alpha_{3}^{2} \mathrm{p}_{1, \perp}^{2} \mathrm{p}_{3, \perp}^{2}-\left(\alpha_{1}-3\right)^{2}\left(\mathrm{p}_{3, \perp}^{2}-\mathrm{p}_{03, \perp}^{2}\right)^{2}}} d \alpha_{3} \mathrm{p}_{3, \perp} d \mathrm{p}_{3, \perp}
\end{aligned}
$$


with the restriction

$$
\alpha_{3}^{2} \mathrm{p}_{1, \perp}^{2} \mathrm{p}_{3, \perp}^{2}-\left(\alpha_{1}-3\right)^{2}\left(\mathrm{p}_{3, \perp}^{2}-\mathrm{p}_{03, \perp}^{2}\right)^{2}>0
$$

and

$$
\cos \tilde{\phi}_{13}=\left(\frac{\alpha_{1}-3}{\alpha_{3}}\right) \frac{\mathrm{p}_{3, \perp}^{2}-\mathrm{p}_{03, \perp}^{2}}{\mathrm{p}_{1, \perp} \mathrm{p}_{3, \perp}}
$$

is the root of $\tilde{M}_{N}^{2}-\tilde{M}_{N}^{(3 N), 2}=0$, with the restriction

$$
\cos ^{2} \tilde{\phi}_{13} \leq 1
$$

From Eq (2.126) it follows that

$$
\tilde{\mathrm{k}}_{1, \perp}=\sqrt{\mathrm{p}_{1, \perp}^{2}+\left(\frac{\beta_{1}}{2} \mathrm{p}_{3, \perp}\right)^{2}-\beta_{1} \mathrm{p}_{1, \perp} \mathrm{p}_{3, \perp} \cos \tilde{\phi}_{13}}
$$

The momenta $\tilde{\mathrm{k}}_{1}$ and $\tilde{\mathrm{k}}_{3}$ are calculated from Eqs. (2.125), and (2.118) respectively.

The 3N SRC nuclear spectral functions [Eq.(3.74)], together with Eqs. (2.125), (2.118), (3.75) to (3.78), and the normalization conditions (2.132), constitute the simplest computational model from which numerical estimates will be calculated in the chapter 4 of the present dissertation. Eqs. (3.65) to (3.73), and (2.132), also constitute a computational model but more calculations are needed to obtain the same numerical estimates.

\subsection{Computational models for SRC nuclear spectral function in nonrelativistic approximation}

The computational models for high momentum nuclear spectral function in the non-relativistic limit of the $\mathrm{VN}$ approximation, $\alpha \approx 1$, with two and three nucleons in short range correlation, are developed in the present section. 


\subsubsection{Computational model for two nucleon SRC nuclear spectral function in nonrelativistic approximation}

The non relativistic $2 \mathrm{~N}$ SRC nuclear spectral functions for proton and neutron are obtained from Eq.(3.4), in the non-relativistic limit of the VN approximation, namely

$$
S_{A, 2 N}^{p / n}\left(\mathrm{p}_{1}, E_{m}\right)=\frac{a_{2}(A, Z)}{\left(2 x_{p / n}\right)^{\gamma}} \int n_{d}\left(\mathrm{p}_{\mathrm{rel}}\right) \Theta\left(\mathrm{p}_{\mathrm{rel}}-\mathrm{k}_{\mathrm{src}}\right) n_{C M}\left(\mathrm{p}_{\mathrm{NN}}\right) \delta\left[E_{m}-E_{m}^{2 N}\right] d \mathbf{p}_{\mathrm{NN}}
$$

where $p / n$ indicates proton and neutron respectively, the parameter $a_{2}(A, Z)$ is defined in Eq. (2.37), $x_{p / n}$ are given by Eq. (3.2), and $n_{d}$ is the deuteron momentum distribution calculated from a nonrelativistic wave function.

The removal energy $E_{m}^{2 N}$ for the 2N SRC [Eq. (2.23)] in the nonrelativistic limit is given by

$$
\begin{aligned}
E_{m}^{2 N} & =E_{t h r}^{(2)}+\frac{\mathrm{p}_{A-2}^{2}}{2\left(M_{A-1}-M_{N}\right)}+\frac{\mathrm{p}_{2}^{2}}{2 M_{N}}-\frac{\mathrm{p}_{1}^{2}}{2 M_{A-1}} \\
& =E_{t h r}^{(2)}+\frac{\mathrm{p}_{N N}^{2}}{2\left(M_{A-1}-M_{N}\right)}+\frac{\left(\mathbf{p}_{N N}-\mathbf{p}_{1}\right)^{2}}{2 M_{N}}-\frac{\mathrm{p}_{1}^{2}}{2 M_{A-1}} \\
& =E_{t h r}^{(2)}+\frac{M_{A-1}-M_{N}}{2 M_{N} M_{A-1}}\left(\mathbf{p}_{1}-\frac{M_{A-1}}{M_{A-1}-M_{N}} \mathbf{p}_{N N}\right)^{2},
\end{aligned}
$$

where $\mathbf{p}_{A-2}=-\mathbf{p}_{N N}, \mathbf{p}_{2}=\mathbf{p}_{N N}-\mathbf{p}_{1}$, and $M_{A-1} \approx M_{A-1}-M_{N}$, have been used. The argument of the delta function in the nuclear spectral function [Eq. (3.79] is therefore given by

$$
E_{m}-E_{m}^{2 N}=\frac{M_{A-1}-M_{N}}{2 M_{N} M_{A-1}}\left(\mathrm{p}_{0 N N}^{2}-\tilde{\mathrm{p}}_{N N}^{2}\right)
$$

where $\mathrm{p}_{0 \mathrm{NN}}$ is

$$
\mathrm{p}_{0 \mathrm{NN}}=\sqrt{2 M_{N} \frac{M_{A-1}}{M_{A-1}-M_{N}}\left(E_{m}-E_{t h r}^{(2)}\right)}
$$

with the restriction

$$
\mathrm{p}_{0 \mathrm{NN}}^{2} \geq 0,
$$


and $\tilde{\mathbf{p}}_{N N}$ is

$$
\tilde{\mathbf{p}}_{N N}=\mathbf{p}_{\mathbf{1}}-\frac{M_{A-1}}{M_{A-1}-M_{N}} \mathbf{p}_{\mathrm{NN}}
$$

The delta function in Eq. (3.79) is integrated over $\mathrm{p}_{\mathrm{NN}}$ by using the change of variables given by Eq. (3.84), hence

$$
2 \pi \mathrm{p}_{\mathrm{NN}}^{2} d \mathrm{p}_{\mathrm{NN}} d \cos \theta_{1 N N}=2 \pi\left(\frac{M_{A-1}-M_{N}}{M_{A-1}}\right)^{3} \tilde{\mathrm{p}}_{N N}^{2} d \tilde{\mathrm{p}}_{N N} d \cos \tilde{\theta}_{1 N N}
$$

where $\tilde{\theta}_{1 N N}$ is the angle between $\mathbf{p}_{\mathbf{1}}$ and $\tilde{\mathbf{p}}_{N N}$.

The integration of the delta function in the nuclear spectral function [Eq. (3.79] yields

$$
\begin{aligned}
\int \delta\left(E_{m}-E_{m}^{2 N}\right) d \mathbf{p}_{\mathrm{NN}} & =2 \pi\left(\frac{M_{A-1}-M_{N}}{M_{A-1}}\right)^{3} \int \delta\left(\mathrm{p}_{0 \mathrm{NN}}^{2}-\tilde{\mathrm{p}}_{\mathrm{NN}}^{2}\right) \tilde{\mathrm{p}}_{N N}^{2} d \tilde{\mathrm{p}}_{N N} d \cos \tilde{\theta}_{1 N N} \\
& =2 \pi M_{N}\left(\frac{M_{A-1}-M_{N}}{M_{A-1}}\right)^{2} \int \mathrm{p}_{0 \mathrm{NN}} d \cos \tilde{\theta}_{1 N N}
\end{aligned}
$$

and therefore

$S_{A, 2 N}^{p / n}\left(\mathrm{p}_{1}, E_{m}\right)=2 \pi M_{N}\left(\frac{M_{A-1}-M_{N}}{M_{A-1}}\right)^{2} \frac{a_{2}(A, Z)}{\left(2 x_{p / n}\right)^{\gamma}} \int_{-1}^{1} n_{d}\left(\mathrm{p}_{\mathrm{rel}}\right) \Theta\left(\mathrm{p}_{\mathrm{rel}}-\mathrm{k}_{\mathrm{src}}\right) n_{C M}\left(\mathrm{p}_{\mathrm{NN}}\right) \mathrm{p}_{0 \mathrm{NN}} d \cos \widetilde{\theta}_{1 N N}$.

The momentum of the correlated nucleons, $\mathrm{p}_{\mathrm{NN}}$, is given by

$$
\begin{aligned}
\mathrm{p}_{\mathrm{NN}} & =\frac{M_{A-1}-M_{N}}{M_{A-1}} \sqrt{\left(\mathbf{p}_{1}-\widetilde{\mathbf{p}}_{N N}\right)^{2}} \\
& =\frac{M_{A-1}-M_{N}}{M_{A-1}} \sqrt{\mathrm{p}_{0 N N}^{2}+\mathrm{p}_{1}^{2}-2 \mathrm{p}_{0 N N} \mathrm{p}_{1} \cos \widetilde{\theta}_{1 N N}},
\end{aligned}
$$

with the restriction

$$
\mathrm{p}_{0 N N}^{2}+\mathrm{p}_{1}^{2}-2 \mathrm{p}_{0 N N} \mathrm{p}_{1} \cos \widetilde{\theta}_{1 N N} \geq 0
$$


The cosine of the angle $\theta_{1 N N}$, between $\mathbf{p}_{1}$ and $\mathbf{p}_{\mathrm{NN}}$, is given by

$$
\begin{aligned}
\cos \theta_{1 N N} & =\frac{M_{A-1}-M_{N}}{M_{A-1} \mathrm{p}_{N N}}\left(\mathrm{p}_{0 N N} \cos \widetilde{\theta}_{1 N N}-\mathrm{p}_{1}\right) \\
& =\frac{\mathrm{p}_{0 N N}^{2}-\mathrm{p}_{1}^{2}-\left(\frac{M_{A-1}}{M_{A-1}-M_{N}}\right)^{2} \mathrm{p}_{\mathrm{NN}}^{2}}{2.0\left(\frac{M_{A-1}}{M_{A-1}-M_{N}}\right) \mathrm{p}_{1} \mathrm{p}_{\mathrm{NN}}}
\end{aligned}
$$

with the restriction

$$
\cos ^{2} \theta_{1 N N} \leq 1
$$

The relative momentum of the correlated pair, $\mathrm{p}_{\text {rel }}$, is

$$
\begin{aligned}
p_{\text {rel }} & =\sqrt{\frac{\left(\mathbf{p}_{\mathbf{1}}-\mathbf{p}_{\mathbf{2}}\right)^{2}}{4}}=\sqrt{\frac{\left(2 \mathbf{p}_{\mathbf{1}}-\mathbf{p}_{\mathrm{NN}}\right)^{2}}{4}} \\
& =\sqrt{\mathrm{p}_{1}^{2}+\frac{\mathrm{p}_{\mathrm{NN}}^{2}}{4}-\mathrm{p}_{1} \mathrm{p}_{\mathrm{NN}} \cos \theta_{1 N N}},
\end{aligned}
$$

with the restriction

$$
\mathrm{p}_{1}^{2}+\frac{\mathrm{p}_{\mathrm{NN}}^{2}}{4}-\mathrm{p}_{1} \mathrm{p}_{\mathrm{NN}} \cos \theta_{1 N N} \geq 0
$$

The cosine of the angle $\theta_{1 \text { rel }}$, between $\mathbf{p}_{1}$ and $\mathbf{p}_{\text {rel }}$, is

$$
\cos \theta_{\text {1rel }}=\frac{2 \mathrm{p}_{1}-\mathrm{p}_{\mathrm{NN}} \cos \theta_{1 \mathrm{NN}}}{2 \mathrm{p}_{\mathrm{rel}}}
$$

with the restriction

$$
\cos ^{2} \theta_{1 \mathrm{rel}} \leq 1
$$

The momentum of the correlated nucleon 2 is given by

$$
\begin{aligned}
\mathrm{p}_{2} & =\sqrt{\left(\mathbf{p}_{\mathbf{1}}-\mathbf{2} \mathbf{p}_{\text {rel }}\right)^{2}} \\
& =\sqrt{\mathrm{p}_{1}^{2}+4 \mathrm{p}_{\mathrm{rel}}^{2}-4 \mathrm{p}_{1} \mathrm{p}_{\mathrm{rel}} \cos \theta_{1 \text { rel }}},
\end{aligned}
$$


with the restrictions

$$
\begin{array}{r}
\mathrm{p}_{1}^{2}+4 \mathrm{p}_{\mathrm{rel}}^{2}-4 \mathrm{p}_{1} \mathrm{p}_{\mathrm{rel}} \cos \theta_{1 \text { rel }}>0, \\
p_{2}>\mathrm{k}_{\mathrm{F}} \sim 0.250 \mathrm{GeV} / \mathrm{c} .
\end{array}
$$

The 2N SRC nuclear spectral functions [Eq.(3.86)], together with Eqs. (2.36), (3.82), and (3.87) to (3.96), and the normalization condition [Eq.(2.35) ]with $\alpha_{1}=1$, constitute the computational model from which numerical estimates are obtained in the chapter 4 of the present dissertation.

\subsubsection{Computational model for three nucleon SRC nuclear spectral function in nonrelativistic approximation}

The non relativistic 3N SRC nuclear spectral functions for proton and neutron are obtained from Eq.(3.20), in the non-relativistic limit of the VN approximation, namely

$$
S_{A, 3 N}^{p / n}\left(\mathrm{p}_{1}, E_{m}\right)=a_{2}^{2}(A) C^{p / n}(A, N) \int n_{d}\left(\mathrm{p}_{3}\right) n_{d}\left(\mathrm{p}_{12}\right) \Theta\left(\mathrm{p}_{3}-k_{s r c}\right) \Theta\left(\mathrm{p}_{12}-k_{s r c}\right) \delta\left(E_{m}-E_{m}^{3 N}\right) d^{3} \mathbf{p}_{3}
$$

where $p / n$ indicates proton and neutron respectively, the factors $C^{p / n}(A, Z)$ by Eq. (3.3), and and $n_{d}$ is the deuteron momentum distribution calculated from a nonrelativistic wave function.

The removal energy $E_{m}^{3 N}$ for the 2N SRC [Eq. (2.38)] in the nonrelativistic limit is given by

$$
\begin{aligned}
E_{m}^{3 N} & =E_{t h r}^{(3)}+\frac{\mathrm{p}_{3}^{2}}{2 M_{N}}+\frac{\mathrm{p}_{2}^{2}}{2 M_{N}}-\frac{\mathrm{p}_{1}^{2}}{2 M_{A-1}} \\
& =E_{t h r}^{(3)}+\frac{\mathrm{p}_{3}^{2}}{2 M_{N}}+\frac{\left(\mathbf{p}_{1}+\mathbf{p}_{3}\right)^{2}}{2 M_{N}}-\frac{\mathrm{p}_{1}^{2}}{2 M_{A-1}} \\
& =\frac{1}{M_{N}}\left[M_{N} E_{t h r}^{(3)}-\left(\frac{2 M_{N}-M_{A-1}}{M_{A-1}}\right) \frac{\mathrm{p}_{1}^{2}}{4}+\left(\frac{\mathbf{p}_{1}}{2}+\mathbf{p}_{3}\right)^{2}\right],
\end{aligned}
$$


where the VN collinear approximation $\mathbf{p}_{1}+\mathbf{p}_{2}+\mathbf{p}_{3}=0$, have been used. The argument of the delta function in the nuclear spectral function [Eq. (3.97] is therefore given by

$$
E_{m}-E_{m}^{3 N}=\frac{1}{M_{N}}\left(\mathrm{p}_{03 N}^{2}-\mathrm{p}_{3 N}^{2}\right)
$$

where $\mathrm{p}_{03 \mathrm{~N}}$ is

$$
p_{03 N}=\sqrt{M_{N}\left(E_{m}-E_{t h r}^{(3)}\right)-\frac{M_{A-1}-2 M_{N}}{M_{A-1}} \frac{\mathrm{p}_{1}^{2}}{4}}
$$

with the restriction

$$
\mathrm{p}_{03 \mathrm{~N}}^{2} \geq 0
$$

and $\mathbf{p}_{\mathbf{3 N}}$ is

$$
\mathbf{p}_{3 N}=\mathbf{p}_{1} / 2+\mathbf{p}_{3}
$$

The delta function in Eq. (3.97) is integrated over $\mathrm{p}_{3}$ by using the change of variables given by Eq. (3.102), hence

$$
\begin{aligned}
\int \delta\left(E_{m}-E_{m}^{3 N}\right) d \mathbf{p}_{3} & =2 \pi \int \delta\left(\mathrm{p}_{03 N}^{2}-\mathrm{p}_{3 N}^{2}\right) \mathrm{p}_{3 N}^{2} d \mathrm{p}_{3 N} d \cos \theta_{13 N} \\
& =\pi M_{N} \int \mathrm{p}_{03 N} d \cos \theta_{13 N}
\end{aligned}
$$

where $\theta_{13 N}$ is the angle between $\mathbf{p}_{1}$ and $\mathbf{p}_{N N}$, and therefore

$S_{A, 3 N}^{p / n}\left(\mathrm{p}_{1}, \mathrm{E}_{\mathrm{m}}\right)=\pi M_{N} a_{2}^{2}(A, y) C^{p / n}(A, Z) \int_{-1}^{1} n_{d}\left(\mathrm{p}_{3}\right) n_{d}\left(\mathrm{p}_{12}\right) \Theta\left(\mathrm{p}_{3}-k_{s r c}\right) \Theta\left(\mathrm{p}_{12}-k_{s r c}\right) \mathrm{p}_{03 N} d \cos \theta_{13 N}$. 
The momenta $\mathrm{p}_{3}$ and $\mathrm{p}_{12}$ are given by

$$
\begin{aligned}
\mathrm{p}_{3} & =\sqrt{\left(\mathbf{p}_{3 N}-\frac{\mathbf{p}_{1}}{2}\right)^{2}} \\
& =\sqrt{\mathrm{p}_{03 \mathrm{~N}}^{2}-\mathrm{p}_{03 \mathrm{~N}} \mathrm{p}_{1} \cos \theta_{13 N}+\frac{\mathrm{p}_{1}^{2}}{4}} \\
\mathrm{p}_{12} & =\sqrt{\left(\frac{\mathbf{p}_{1}-\mathbf{p}_{2}}{2}\right)^{2}}=\frac{1}{2} \sqrt{\left(\mathbf{p}_{3 N}+\frac{3 \mathbf{p}_{1}}{2}\right)^{2}} \\
& =\frac{1}{2} \sqrt{\mathrm{p}_{03 \mathrm{~N}}^{2}+3 \mathrm{p}_{03 \mathrm{~N}} \mathrm{p}_{1} \cos \theta_{13 N}+\frac{9 \mathrm{p}_{1}^{2}}{4}},
\end{aligned}
$$

with the restriction

$$
\mathrm{p}_{03 \mathrm{~N}}^{2}-\mathrm{p}_{03 \mathrm{~N}} \mathrm{p}_{1} \cos \theta_{13 N}+\frac{\mathrm{p}_{1}^{2}}{4} \geq 0
$$

The cosine of the angle $\theta_{13}$, between $\mathbf{p}_{\mathbf{1}}$ and $\mathbf{p}_{\mathbf{3}}$, is

$$
\cos \theta_{13}=\frac{2 \mathrm{p}_{03 \mathrm{~N}} \cos \theta_{13 N}-\mathrm{p}_{1}}{2 \mathrm{p}_{3}},
$$

with the restriction

$$
\cos ^{2} \theta_{13} \leq 1
$$

The cosine of the angle $\theta_{112}$, between $\mathbf{p}_{1}$ and $\mathbf{p}_{\mathbf{1 2}}$, is

$$
\cos \theta_{112}=\frac{3 \mathrm{p}_{1}+2 \mathrm{p}_{03 \mathrm{~N}} \cos \theta_{13 \mathrm{~N}}}{4 \mathrm{p}_{12}}
$$

with the restriction

$$
\cos ^{2} \theta_{112} \leq 1
$$


The momentum of the correlated nucleon 2 is

$$
\begin{aligned}
\mathrm{p}_{2} & =\sqrt{\left(\mathbf{p}_{\mathbf{1}}-\mathbf{2} \mathbf{p}_{\mathbf{1 2}}\right)^{2}} \\
& =\sqrt{\mathrm{p}_{1}^{2}+4 \mathrm{p}_{12}^{2}-2 \mathrm{p}_{1} \mathrm{p}_{12} \cos \theta_{112}},
\end{aligned}
$$

with the restrictions

$$
\begin{array}{r}
\mathrm{p}_{1}^{2}+4 \mathrm{p}_{12}^{2}-2 \mathrm{p}_{1} \mathrm{p}_{12} \cos \theta_{112} \geq 0 \\
\mathrm{p}_{2}>\mathrm{k}_{\mathrm{F}} \sim 0.250 \mathrm{GeV} / \mathrm{c} .
\end{array}
$$

The 3N SRC nuclear spectral functions (3.104), together with Eqs. (3.100), and (3.105) to (3.113), and the normalization condition (2.48) with $\alpha_{1}=1$, constitute the computational model from which numerical estimates will be calculated in chapter 4 of the present dissertation.

\subsection{Summary}

The computational models for $2 \mathrm{~N}$ and $3 \mathrm{~N}$ SRC high momentum nuclear spectral functions in $\mathrm{VN}$ and LF approximation, as well as in the non-relativistic limit of the VN approximation, have been obtained in the present chapter. The computational models have been developed by integration of the delta functions in the mathematical models of chapter 2.

The computational models are a set of computable integrals that represent the high momentum nuclear spectral functions, as well as equations for momenta and cosine of angles between momentum vectors, and for auxiliary variables. The restrictions in the values of momenta and cosine of angles have been also defined to ensure that the results are physically valid. The computational models are used to obtain the numerical estimates of the nuclear spectral functions, density matrices and momentum distributions, given in the next chapter of the present dissertation. 


\section{CHAPTER 4}

\section{Numerical estimates of nuclear spectral functions, density matrices and momentum distributions}

The computational models developed in chapter 3 of the present dissertation are used to obtain numerical estimates of $2 \mathrm{~N}$ and $3 \mathrm{~N}$ SRC nuclear spectral functions for $\mathrm{LF}, \mathrm{VN}$ and VN in the nonrelativistic limit approximations. The numerical estimates for the LF density matrix for light and heavy nuclei are also included. The numerical estimates and the parametrization of the momentum distribution of the bound nucleon are also presented. The momenta distribution estimates are obtained by the integration of the corresponding nuclear spectral functions for $\mathrm{VN}$ and $\mathrm{VN}$ in the nonrelativistic limit approximations.

The outline of the chapter is as follows. Section 4.1 contains a brief discussion about the range of validity and set of parameters which are used for the numerical estimates of the the nuclear spectral functions. The deuteron momentum distribution used in the nuclear spectral function calculations is described in section 4.2, which also includes the numerical estimates for the deuteron LF density matrix. The numerical estimates for the nuclear spectral functions in $\mathrm{VN}, \mathrm{VN}$ in the nonrelativistic limit, and LF approximations are included in the sections 4.3 and 4.4 respectively. The section 4.5 includes the numerical estimates for the SRC density matrices in LF approximation. The section 4.6 presents the numerical estimates for the SRC momentum distribution in $\mathrm{VN}$ approximation, and $\mathrm{VN}$ approximation in the nonrelativistic limit. The section 4.6 also contains the numerical estimates for the SRC nonrelativistic momentum distributions for light nuclei $(\mathrm{A} \leq 12)$ which are compared with ab initio quantum Monte Carlo calculations $[44,59,65,67,69,71]$, and for some heavy nuclei $(\mathrm{A}>12)$ with the calculations for proton given in [52].

The parametrization of the SRC and the mean field momentum distributions in VN and VN in the nonrelativistic approximations are presented in section 4.7. The section 4.8 summarizes the results of the chapter. 


\subsection{Parameters for numerical estimates of the nuclear spectral functions}

Before presenting the numerical estimates of the nuclear spectral functions, it is convenient to discuss briefly the range of validity and set of parameters which will be used to obtain such numerical estimates.

The main assumption for the nuclear spectral function models is the dominance of NN SRC in the nuclear dynamics for internal momenta $\mathrm{p} \gtrsim \mathrm{k}_{\mathrm{src}}>\mathrm{k}_{\mathrm{F}}$, where $\mathrm{k}_{\mathrm{src}}$ is the relative momentum threshold at which a NN system with such relative momentum can be considered in the short-range correlation. The next major assumption is the dominance of the isosinglet $p n$ component in the NN SRC. The empirical evidence of the dominance of NN SRCs was accumulated during the last several decades (see e.g. $[43,49,64,74,78,84,103])$ in high energy electroand hadroproduction reactions. Recent triple-coincident experiments [85, 90, 119] indicated that the $p n$ dominance in the nucleon-nucleon SRC persists for up to the heavy nuclei such as $A=208$. Hence, it is expected that the models developed in the present dissertation should be valid for a wide range of atomic nuclei.

The parameters discussed below are independent of the use of the VN or LF approximations. Therefore, further refinements are achieved in their values for lightest nuclei $(A \leq 12)$, by considering the nonrelativistic limit of the $\mathrm{VN}$ approximation model and comparing them with $a b$ initio calculations that result from the variational Monte Carlo methods given in [120]. These refinements are presented in section 4.3.

With the parameters for $2 \mathrm{~N}$ and 3N SRCs fixed, the normalization factors $n_{2 N}^{N}$ and $n_{3 N}^{N}$ within VN and LF approximations can be calculated. Note that the normalization factors will be model dependent since the $2 \mathrm{~N}$ and $3 \mathrm{~N}$ momentum distributions predicted in $\mathrm{VN}$ and $\mathrm{LF}$ approximations are different. Once these normalizations are calculated, the norm of the mean

field distributions can be estimated from the relation $n_{M F}^{N}=1-n_{2 N}^{N}-n_{3 N}^{N}$. Thus the estimates for the normalization of mean field distributions will be indirectly VN or LF model dependent. 


\subsubsection{Parameter $a_{2}(A, Z)$}

The most important parameter that defines the strength of $2 \mathrm{~N} \mathrm{SRC}$ is $a_{2}(A, Z)$. Within the short-range correlation framework, the parameter can be extracted from the ratios of the cross sections of high momentum transfer inclusive electronuclear scattering off nuclei $A$ and the deuteron $[43,49]$. Recent measurements at Jefferson $\operatorname{Lab}[78,84,103]$ provided the magnitudes of $a_{2}(A, Z)$ for a rather wide spectrum of atomic nuclei.

\subsubsection{Relative momentum threshold $k_{s r c}$}

The 2N SRC relative momentum threshold, $k_{s r c}$, defines the momentum distribution of NN SRC in Eqs. (2.37) and (2.99). The value of $k_{s r c}$ is fixed from the condition that it ishould be sufficiently large for mean-field contribution to be insignificant, as well as close to the threshold value for which $p n$ dominance is observed empirically [85, 90, 119]. Another condition in defining $k_{s r c}$ is the onset of the dominance of the $D$-wave contribution in the high momentum part of the deuteron wave function as described in section 4.2 below. The conditions imply a value for $k_{s r c} \sim 400 \mathrm{MeV} / c$ to be applied for the calculation of numerical estimates of the SRC nuclear spectral function.

\subsubsection{Parameter $\gamma$}

In the nuclear spectral function model developed in the present dissertation, the contributions of $p p$ and $n n$ SRCs were neglected, however they are expected to increase with the mass number A [112]. To account for the effects due to the $p p$ and $n n$ SRCs correlations, the parameter $\gamma$ is introduced. On the basis of the experimental observation [119] that in the 2N SRC regions $p n$ dominates by almost a factor of 20 for a wide range of nuclei (up to $A=208$ ), the following approximations are adopted: $\gamma \approx 0.8$ for asymmetric nuclei, and $\gamma \approx 1.0$ for symmetric nuclei. 


\subsubsection{Parameters for 2N SRC center of mass momentum distribution}

The width of the $2 \mathrm{~N}$ SRC center of mass distribution, $\beta(A)$, and the parameter $N_{0}$ in Eq. (2.36), are chosen from the estimates that result from the convolution of the mean-field distribution of two independent nucleons presented in Ref. [52].

\subsubsection{N SRC suppression factor $C^{N}(A, Z)$}

For the case of 3N SRCs, the only additional parameter needed to estimate the nuclear spectral function is the suppression factor $C^{N}(A, Z)$ defined in Eq. (2.49). The factor $C^{N}(A, Z)$ accounts for the suppression of the $3 \mathrm{~N}$ configurations with two identical spectators like $p p$ and $n n$ pairs, and affects only the distribution of the minority component in the asymmetric nucleus. For example, according to the considered model, the neutron can not be generated from 3N SRC in the ${ }^{3} \mathrm{He}$ nucleus, since it will produce two "parallel" protons in the final state. The factors $C^{p}(A, Z)$ and $C^{n}(A, Z)$ are defined in Eqs. (3.3) for proton and neutron respectively.

\subsection{Momentum distribution and light-front density matrix for deuteron}

The numerical estimates of the momentum distribution and the light-front density matrix for deuteron are included in the present section,

\subsubsection{Momentum distribution for deuteron}

The D-wave deuteron momentum distribution $\left(n_{d}\right)$ used for the nuclear spectral function calculations, results from a wave function modeled with the Argone V18 (AV18) two-nucleon potential, that is specially suitable for the spectral function model since fits both $p p$ and $n p$

data [51]. The total momentum distribution, as well as the S-wave and the D-wave momentum distribution components, calculated with the AV18 potential is shown in Fig. 4.1 [69]. 


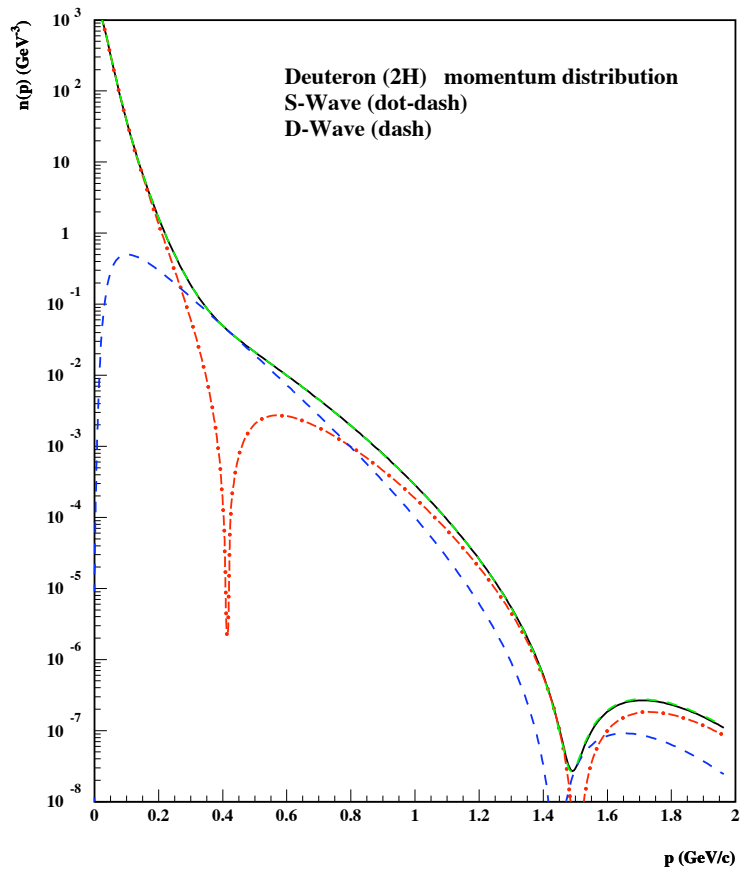

Figure 4.1: The momentum distribution of deuteron (s-wave: dot-dash, d-wave:dash) .

The D-wave of the deuteron momentum distribution, shown in Fig. 4.1, starts to dominate (by a factor of $\approx 8$ ) the $\mathrm{S}$-wave momentum distribution at $\mathrm{p} \approx 350 \mathrm{MeV} / \mathrm{c}$, and for $\mathrm{p} \approx 400$ $\mathrm{MeV} / \mathrm{c}$ is more than two orders of magnitude greater than the S-wave momentum distribution. Hence, for a proper modeling of the D-wave deuteron momentum distribution $\left(n_{d}\right)$, the value of $400 \mathrm{MeV} / \mathrm{c}$ for the $2 \mathrm{~N} \mathrm{SRC} \mathrm{relative} \mathrm{momentum} \mathrm{threshold,} k_{s r c}$, was chosen for the numerical estimates of the nuclear spectral function.

\subsubsection{Light-front density matrix for deuteron}

The numerical estimates for light-front density matrix of the deuteron used for the LF nuclear spectral function calculations, was obtained by using the model for this density matrix given by Eq. (3.37). The graph for the density matrix as well as the graph of the momentum distribution used in its calculation is given in Fig. 4.2. 


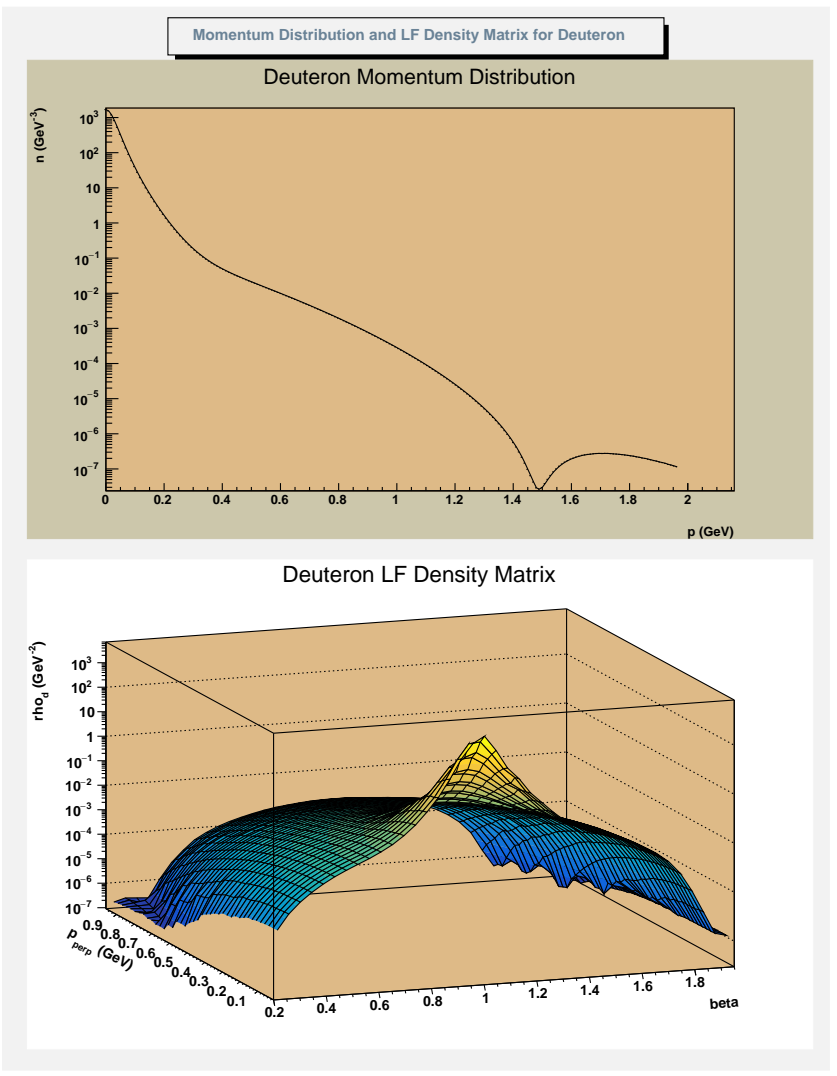

Figure 4.2: Upper graph: Deuteron momentum distribution. Lower graph: Deuteron lightfront density matrix

The graph shows that the deuteron density matrix is the joint probability of the transverse momentum $\left(\mathrm{p}_{\perp}\right)$ and the momentum fraction of the $2 \mathrm{~N}$ SRC, $\beta$, carried by the bound nucleon, with a maximum value for $\beta=1$, as it is expected. The momentum distribution graph reproduces the total momentum distribution showed in the Fig. 4.1 above, as it was also expected.

\subsection{Numerical estimates for SRC nuclear spectral function in virtual nucleon approximation}

The $2 \mathrm{~N}$ and $3 \mathrm{~N}$ SRC nuclear spectral functions for proton and neutron in $\mathrm{VN}$ approximation are calculated with the computational models given by Eqs.(3.8) to (3.19), and Eqs.(3.26) to (3.35) respectively. The same calculations for $2 \mathrm{~N}$ and $3 \mathrm{~N}$ SRC nuclear spectral function in VN approximation in the nonrelativistic limit are made with Eqs.(3.82) to (3.96), and with Eqs.(3.100) to 
(3.113) respectively. The SRC parameters for the calculation of the nuclear spectral functions are given in the Tables 4.1 and 4.2 described in the section 4.6 of the present chapter.

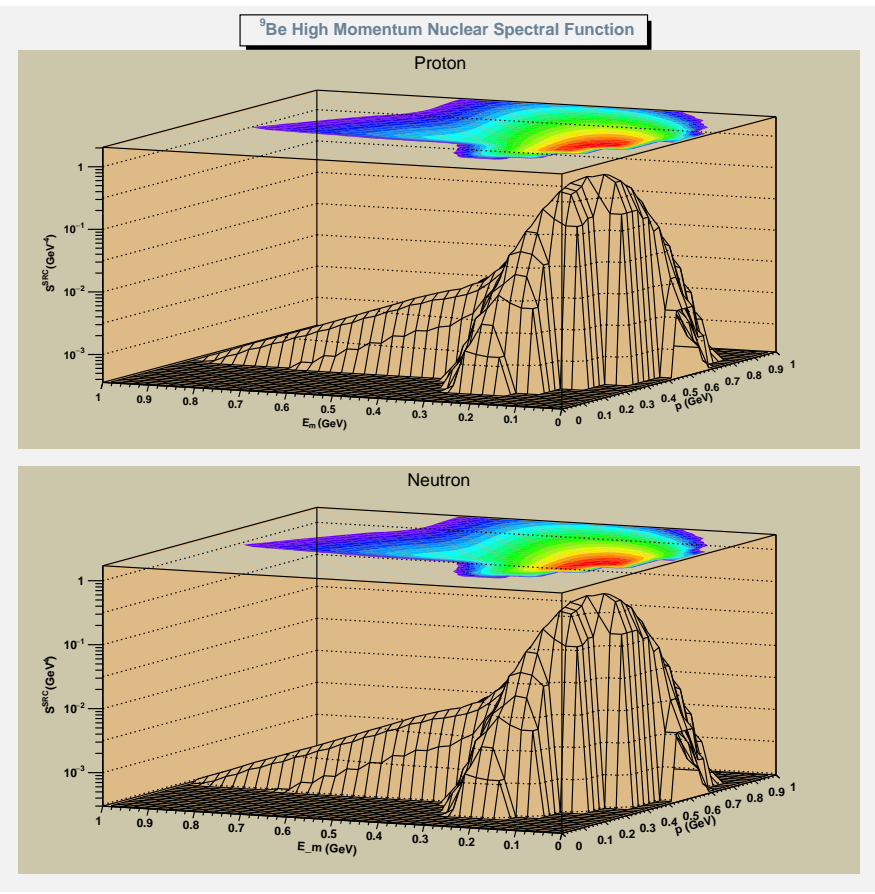

Figure 4.3: Nuclear spectral function for ${ }^{9} \mathrm{Be}$ in the nonrelativistic limit of the VNA

The numerical results for nuclear spectral function for ${ }^{9} \mathrm{Be}$ and ${ }^{56} \mathrm{Fe}$ in the non relativistic limit of the VN approximation are represented by the three dimensional graphs depicted in Figs. 4.3 and 4.4 respectively. The graphs show that the nuclear spectral function as expected, is the joint probability of the momentum (p) and removal energy $\left(\mathrm{E}_{\mathrm{m}}\right)$ of the bound nucleon. 


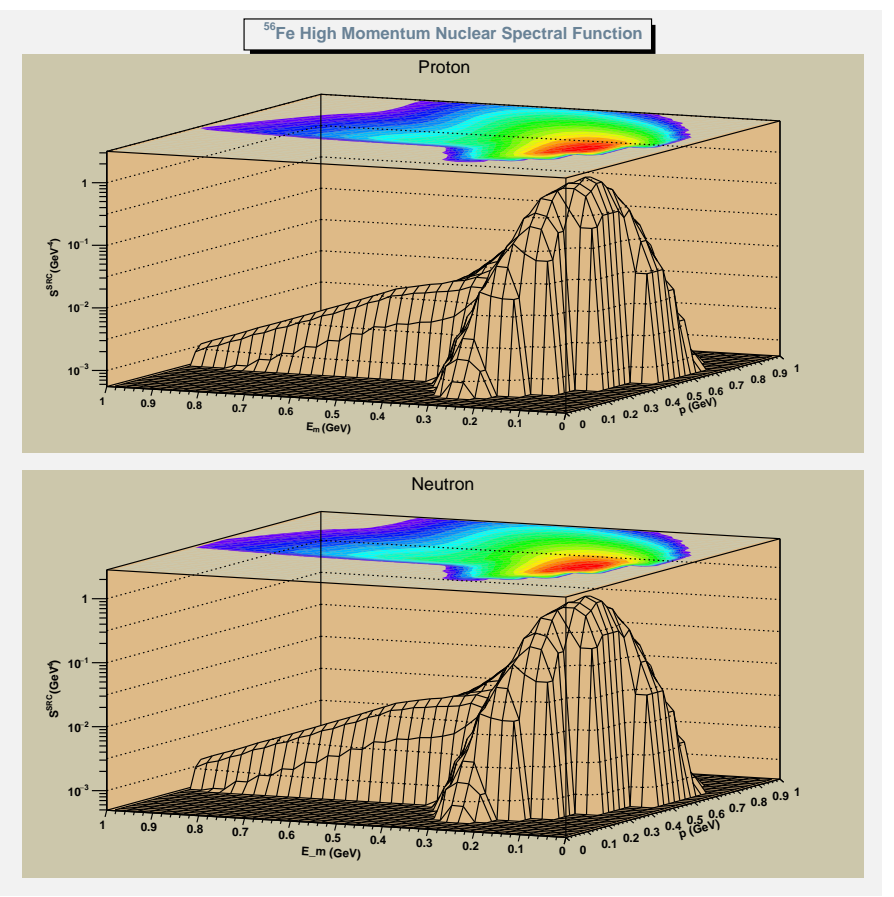

Figure 4.4: Nuclear spectral function for ${ }^{56} \mathrm{Fe}$ in the nonrelativistic limit of the VNA

The spectral function graphs also present a region of maximum values for $0.3 \leq p \leq 0.6$ $\mathrm{GeV} / \mathrm{c}$, that, as expected, corresponds to the dominance of the D-wave of the deuteron momentum distribution (see Fig. 4.1), over the S-wave momentum distribution, that implies maximum probability of having 2N SRC in the nucleus.

The graphs for the numerical estimates of nuclear spectral function with constant values of momentum for VNA and VNA in the nonrelativistic limit, are shown in Fig. 4.5 for ${ }^{3} \mathrm{He}$ and ${ }^{9} \mathrm{Be}$. The spectral function for proton in ${ }^{3} \mathrm{He}$, and for proton and neutron in ${ }^{9} \mathrm{Be}$ shows a tail for $\mathrm{p}>0.45 \mathrm{GeV} / \mathrm{c}$ due to the presence of $3 \mathrm{~N}$ SRC for these nucleons. This tail is not present for the spectral function for the neutron in the ${ }^{3} \mathrm{He}$ nucleus, due to the fact that $3 \mathrm{~N}$ SRC is not allowed for this nucleon due to the Pauli exclusion principle. The maximum value of the spectral function has a monotonic decrease with the values of momentum, also, in general, the dispersion of the removal energy $\mathrm{E}_{m}$ increases when the momentum increases. 

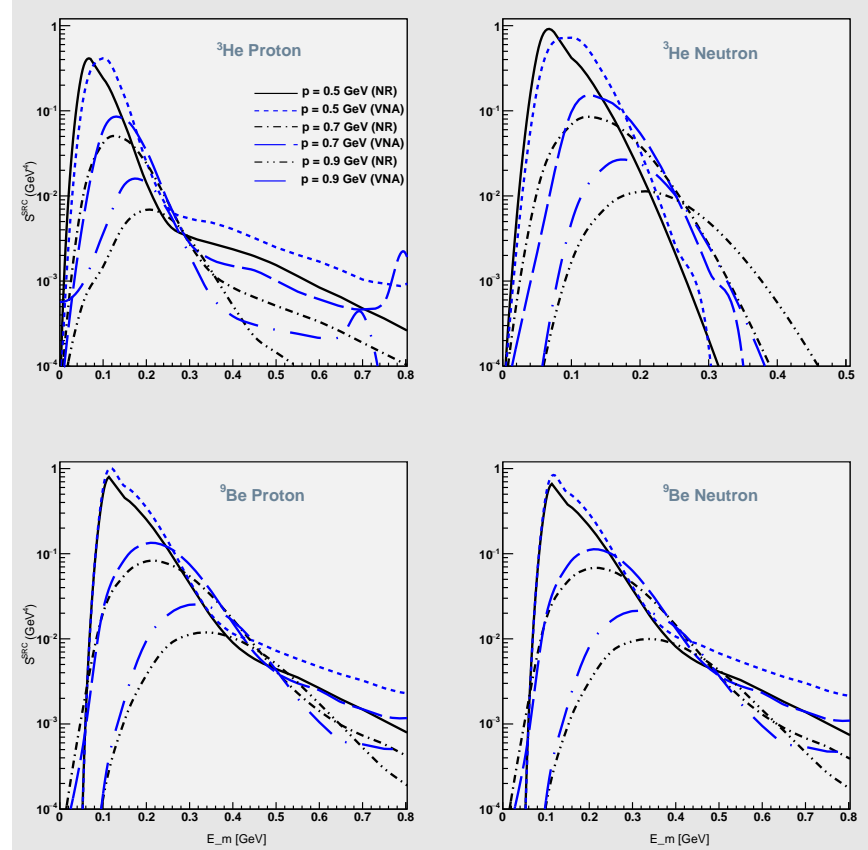

Figure 4.5: Nuclear spectral function, with constant values of momentum for ${ }^{3} \mathrm{He}$ and ${ }^{9} \mathrm{Be}$, in VNA and in the nonrelativistic limit of the VNA

\subsection{Numerical estimates for SRC nuclear spectral function in light-front approximation}

The 2N and 3N SRC nuclear spectral functions for proton and neutron in the LF approximation are calculated with the computational models given by Eq.(3.54) (to (3.58), and Eqs.(3.74) to (3.78) respectively. The SRC parameters for the calculation of the spectral function are given in Tables 4.1 and 4.2 described in the section 4.6 of the present chapter. 


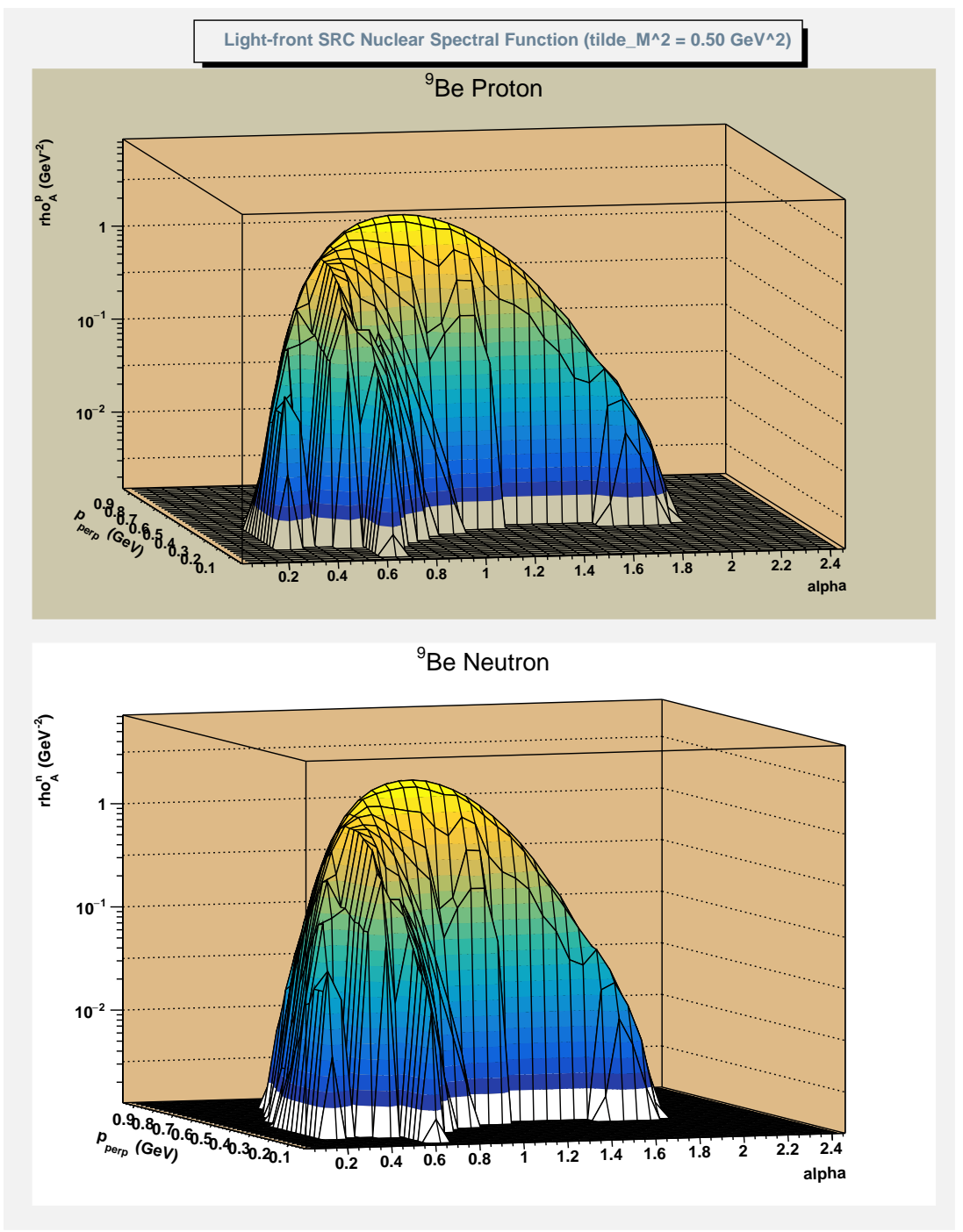

Figure 4.6: Nuclear spectral function for ${ }^{9} \mathrm{Be}$ in light-front approximation, for $\tilde{M}_{N}^{2}=0.5 \mathrm{GeV}^{2}$

The numerical results for the SRC nuclear spectral function for ${ }^{9} \mathrm{Be}$ in the LF approximation are represented by the three dimensional graphs depicted in Fig. 4.6, for an invariant mass $\tilde{M}_{N}^{2}=0.5 \mathrm{GeV}^{2}$. A similar graph is shown in Fig. 4.7, for the SRC nuclear spectral function for ${ }^{197} \mathrm{Au}$ in the LF approximation, for an invariant mass $\tilde{M}_{N}^{2}=0.2 \mathrm{GeV}^{2}$. The graphs show that the LF nuclear spectral function for constant values of the invariant mass, is the joint probability of finding a bound nucleon in the nucleus with light-front "+" momentum fraction $(\alpha)$ and transverse momentum $\left(\mathrm{p}_{\perp}\right)$, as it is expected,. 


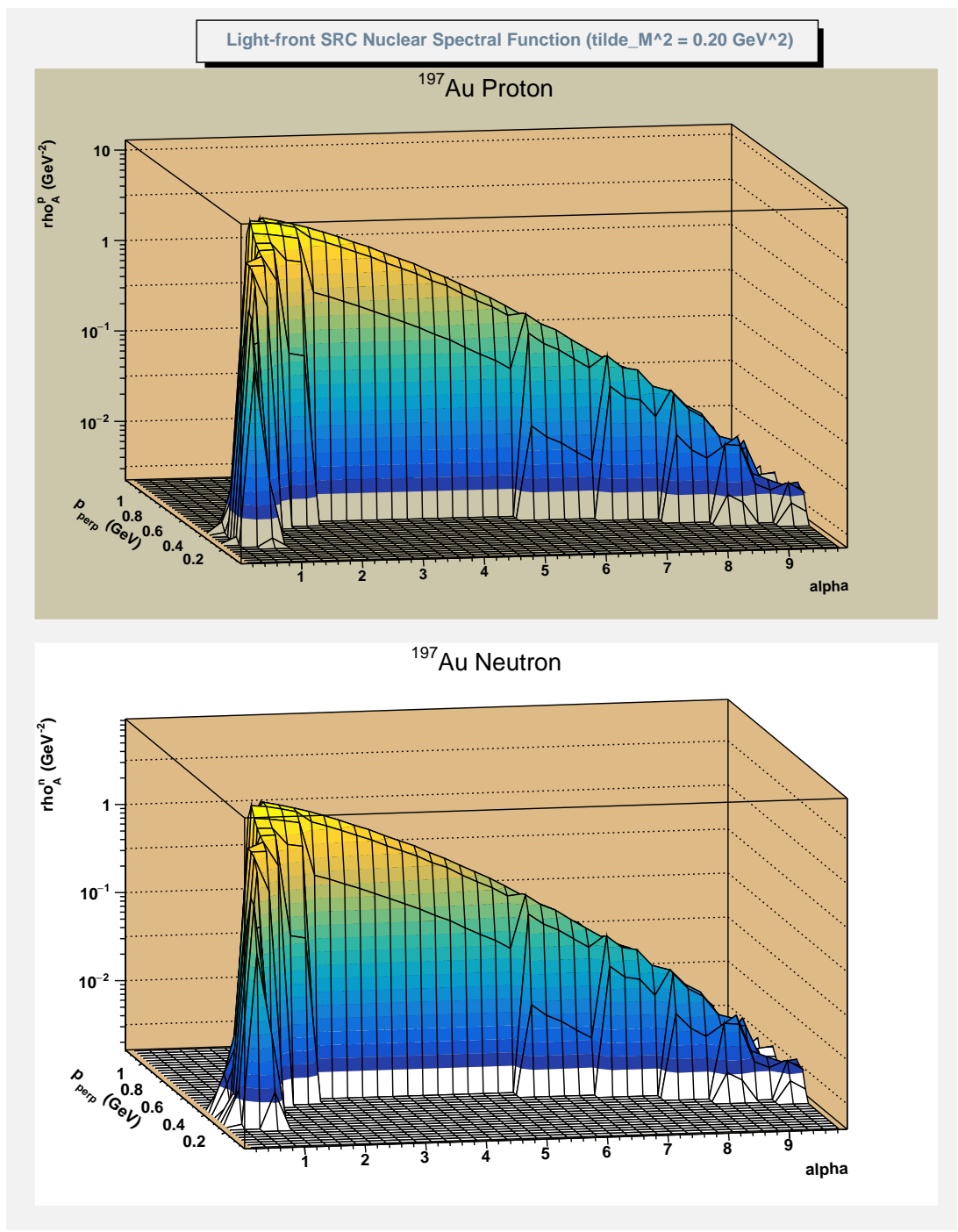

Figure 4.7: Nuclear spectral function for ${ }^{197} \mathrm{Au}$ in light-front approximation, for $\tilde{M}_{N}^{2}=0.2 \mathrm{GeV}^{2}$

The SRC nuclear spectral function graphs show that the range of values of $\alpha$ for which the spectral function is greater than zero, increases with the value of the mass number $A$ of the nucleus. The graphs also show that the maximum values of the LF nuclear spectral function for ${ }^{9} \mathrm{Be}$ are in the range $0.8<\alpha<1.2$, whereas for ${ }^{197} \mathrm{Au}$ are in the range $1.0<\alpha<3.0$, so that the range also increases with the mass number $A$ of the nucleus. The results are as expected, since the range of possible values of the light-front "+" momentum fraction for any nucleus with mass number $A$ is $0<\alpha<A$. 


\subsection{Numerical estimates for SRC density matrices in light-front approximation}

The SRC density matrices for proton and neutron in the LF approximation are calculated with Eq. (2.54), on which the density matrix is obtained by the integration of the SRC nuclear spectral function in the LF approximation over the invariant mass $\tilde{M}_{N}^{2}$.

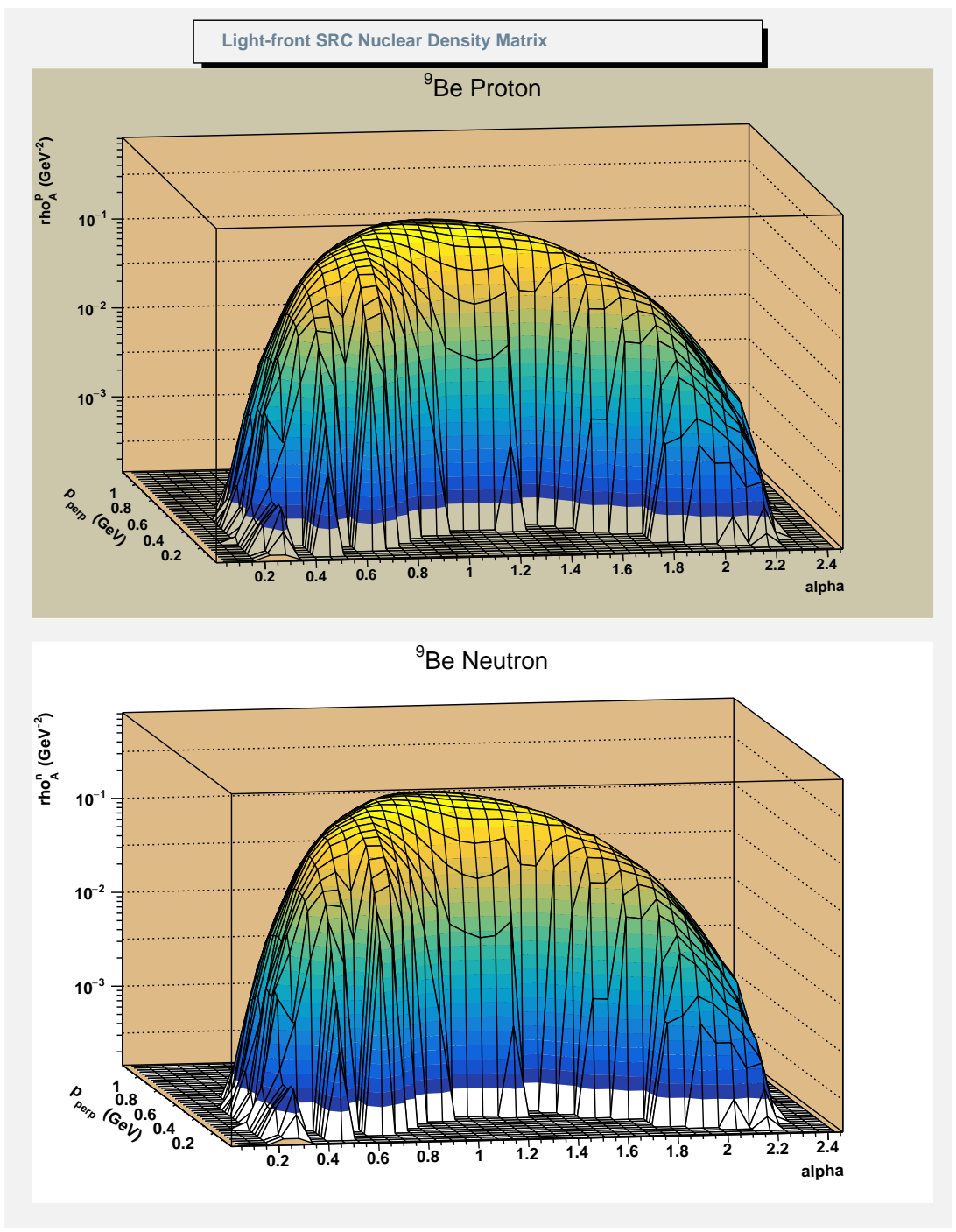

Figure 4.8: SRC density matrix for ${ }^{9} \mathrm{Be}$ in light-front approximation

The numerical results for the SRC density matrices for ${ }^{9} \mathrm{Be}$ and ${ }^{197} \mathrm{Au}$ in the LF approximation are represented by the three dimensional graphs depicted in Figs. 4.8 and 4.9. The graphs show that the LF SRC density matrix is the joint probability of finding a bound nucleon in the 
nucleus with the light-front "+" momentum fraction $(\alpha)$ and the transverse momentum $\left(\mathrm{p}_{\perp}\right)$, as it is expected.
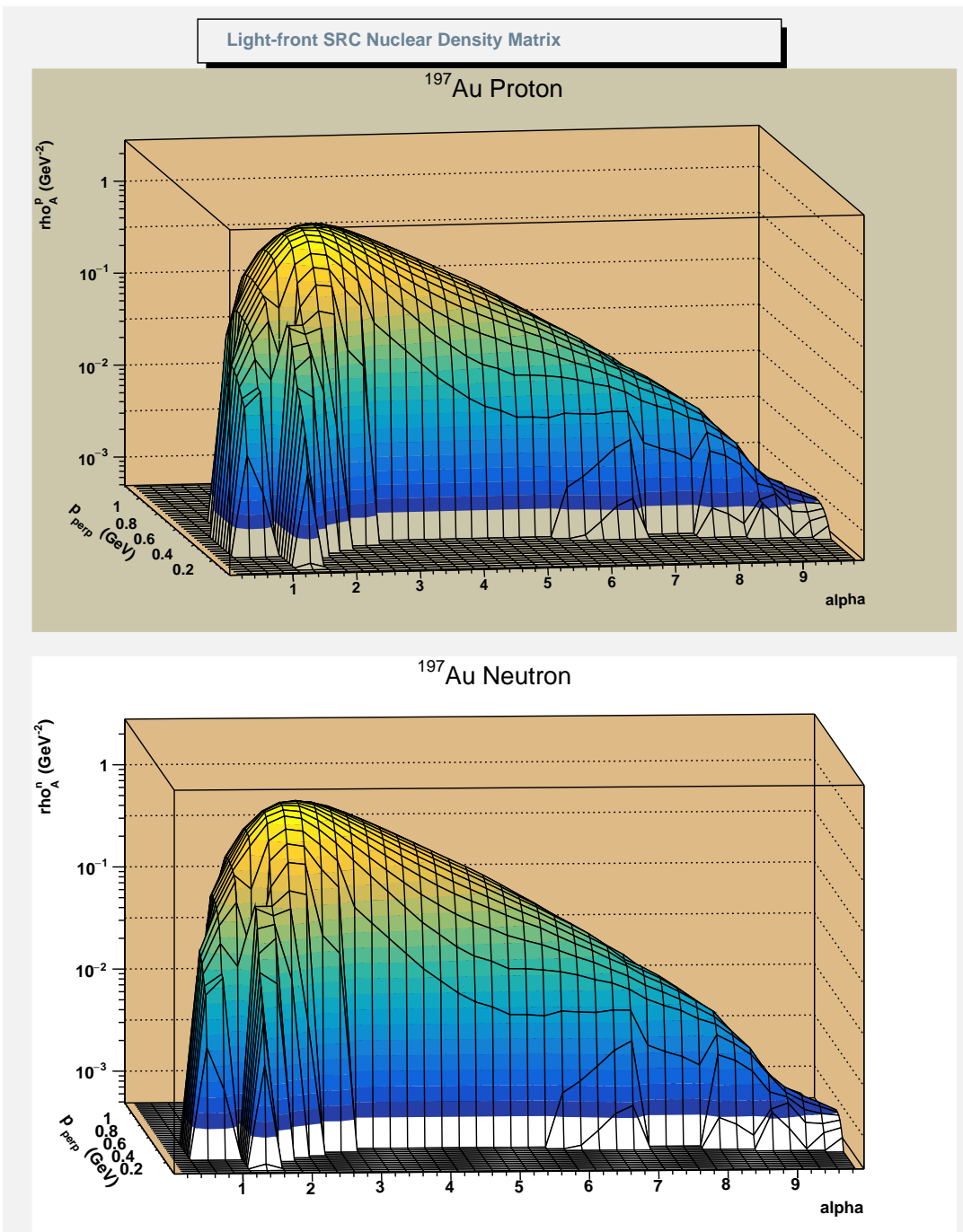

Figure 4.9: SRC density matrix for ${ }^{197} \mathrm{Au}$ in light-front approximation

Similar to the case of the SRC nuclear spectral function, the graphs for the SRC density matrices show that the range of values of $\alpha$ for which the spectral function is greater than zero, increases with the value of the mass number $A$ of the nucleus. The graphs also show that the maximum values of the LF nuclear spectral function for ${ }^{9} \mathrm{Be}$ are in the range $0.8<\alpha<1.2$, whereas for ${ }^{197} \mathrm{Au}$ are in the range $1.0<\alpha<3.0$, so that the range also increases with the mass 
number $A$ of the nucleus. The results are as expected, since the range of possible values of the light-front "+" momentum fraction for any nucleus with mass number $A$ is $0<\alpha<A$.

\subsection{Numerical estimates for SRC momentum distribution in virtual nucleon approximation}

The numerical estimates for $2 \mathrm{~N}$ and $3 \mathrm{~N}$ SRC momentum distribution for proton and neutron are calculated with the following equations:

$$
\begin{aligned}
& n_{A, 2 N}^{p / n}\left(\mathrm{p}_{1}\right)=\int_{0}^{1} S_{A, 2 N}^{p / n}\left(\mathrm{p}_{1}, E_{m}\right) d E_{m} \\
& n_{A, 3 N}^{p / n}\left(\mathrm{p}_{1}\right)=\int_{0}^{1} S_{A, 3 N}^{p / n}\left(\mathrm{p}_{1}, E_{m}\right) d E_{m}
\end{aligned}
$$

where the nuclear spectral functions $S_{A, 2 N}^{p / n}$ and $S_{A, 3 N}^{p / n}$ are given by Eqs. (3.13) and (3.29) for the VN approximation approach respectively, and by Eqs. (3.86) and (3.104) for the VN approximation in the nonrelativistic limit respectively.

The SRC parameters for the calculation of the spectral function are given in Tables 4.1 and 4.2, where the auxiliary parameters $a_{2 \mathrm{p}(\mathrm{n}) \text {-eff }}, a_{3 \mathrm{p}(\mathrm{n})}$, and $a_{3 \mathrm{p}(\mathrm{n}) \text {-eff }}$ have been included to simplify the notations. The equations used to calculate the auxiliary parameters are

$$
\begin{aligned}
a_{2 \mathrm{p}(\mathrm{n}) \text {-eff }} & =\frac{a_{2}(A, Z)}{\left(2 X_{p(n)}\right)^{\gamma}} \\
a_{3 \mathrm{p}} & =a_{2}^{2}(A, Z) f_{p} \\
a_{3 \mathrm{n}} & =a_{3 \mathrm{p}} f_{n} \\
a_{3 \mathrm{p}-\mathrm{eff}} & =a_{3 p} C^{p}(A, Z) \\
a_{3 \mathrm{n}-\mathrm{eff}} & =a_{3 n} C^{n}(A, Z)
\end{aligned}
$$

where $\mathrm{p}(\mathrm{n})$ stands for proton and neutron respectively, $a_{2}(A, Z)$ is defined in Eq. (2.37), $x_{p / n}$ are given by Eq. (3.2), and the $C^{p / n}(A, N)$ factors by Eq. (3.3). The proportionality factors $\mathrm{f}_{p / n}$ were chosen to verify the prediction that the overall probability of finding 3N SRC in the nu- 
cleus is proportional to the factor $a_{2}^{2}(A, Z)$ (Eq. (2.49). The expected range of values for the proportionality factors was defined as $.85 \leq f_{p / n} \leq 1.15$.

Table 4.1: 2N SRC parameters for light and heavy Nuclei $\left(\mathrm{k}_{\mathrm{F}}\right.$ in $\left.\mathrm{GeV}\right)$

\begin{tabular}{|c||c|c|c|c|c|c|c|}
\hline Nucleus & $a_{2}$ & $a_{\text {2peff }}$ & $a_{\text {2neff }}$ & $a_{\text {2exp }}[103]$ & $\alpha_{c m}$ & $\gamma$ & $\mathrm{k}_{\mathrm{F}}$ \\
\hline${ }^{3} \mathrm{He}$ & 1.65 & 1.31 & 2.28 & 2.13 & 3.70 & 0.80 & 0.15 \\
${ }^{4} \mathrm{He}$ & 3.10 & 3.10 & 3.10 & 3.60 & 2.40 & 1.00 & 0.16 \\
${ }^{6} \mathrm{He}$ & 2.35 & 3.25 & 1.87 & & 2.20 & 0.80 & 0.18 \\
${ }^{8} \mathrm{He}$ & 2.10 & 3.66 & 1.52 & & 2.20 & 0.80 & 0.20 \\
${ }^{6} \mathrm{Li}$ & 2.75 & 2.75 & 2.75 & & 2.20 & 1.00 & 0.20 \\
${ }^{7} \mathrm{Li}$ & 2.72 & 3.08 & 2.44 & & 2.10 & 0.80 & 0.20 \\
${ }^{8} \mathrm{Li}$ & 2.60 & 3.27 & 2.17 & & 2.00 & 0.80 & 0.20 \\
${ }^{9} \mathrm{Li}$ & 2.65 & 3.67 & 2.11 & & 1.90 & 0.80 & 0.20 \\
${ }^{8} \mathrm{Be}$ & 3.40 & 3.40 & 3.40 & & 1.50 & 1.00 & 0.20 \\
${ }^{9} \mathrm{Be}$ & 3.10 & 3.41 & 2.85 & 3.91 & 1.20 & 0.80 & 0.20 \\
${ }^{10} \mathrm{Be}$ & 2.90 & 3.47 & 2.50 & & 1.10 & 0.80 & 0.22 \\
${ }^{10} \mathrm{~B}$ & 3.00 & 3.00 & 3.00 & & 1.10 & 1.00 & 0.22 \\
${ }^{11} \mathrm{~B}$ & 3.20 & 3.45 & 2.98 & & 1.10 & 0.80 & 0.22 \\
${ }^{12} \mathrm{C}$ & 4.25 & 4.25 & 4.25 & 4.75 & 1.00 & 1.00 & 0.221 \\
${ }^{16} \mathrm{O}$ & 4.20 & 4.20 & 4.20 & & 1.20 & 1.00 & 0.220 \\
${ }^{27} \mathrm{Al}$ & 4.50 & 4.64 & 4.37 & & 1.00 & 0.80 & 0.235 \\
${ }^{40} \mathrm{Ca}$ & 4.40 & 4.40 & 4.40 & & 1.00 & 1.00 & 0.251 \\
${ }^{56} \mathrm{Fe}$ & 4.95 & 5.25 & 4.68 & & 1.10 & 0.80 & 0.256 \\
${ }^{64} \mathrm{Cu}$ & 5.02 & 5.43 & 4.67 & 5.21 & 1.00 & 0.80 & 0.260 \\
${ }^{197} \mathrm{Au}$ & 4.56 & 5.44 & 3.95 & 5.16 & 1.00 & 0.80 & 0.265 \\
${ }^{208} \mathrm{~Pb}$ & 4.80 & 5.80 & 4.12 & & 1.00 & 0.80 & 0.265 \\
\hline
\end{tabular}

The criteria used to estimate the values of $a_{2}$ and $a_{3}$ for light nuclei (A $\left.\leq 12\right)$ was that, in the momentum range $0.45<\mathrm{p}<1.00 \mathrm{GeV} / \mathrm{c}$, the allowed maximum deviation of the momentum distribution numerical estimates, obtained with Eq. 4.1, from the quantum Monte Carlo (QMC) results given in [69] was of $\pm 15 \%$. For nuclei with experimental values, $a_{2 e p}$ for $a_{2}$, the values $a_{2}$ were chosen to be, up to $30 \%$, equal to the experimental values given in references [84, 103]. For heavier nuclei with $\mathrm{A}>12$, the values of $a_{2}$ were chosen equal to the available experimental values in $[84,103]$. 
Table 4.2: 3N SRC parameters for light and heavy nuclei

\begin{tabular}{|c||c|c|c|c|c|c|}
\hline Nucleus & $f_{p}$ & $a_{3 \mathrm{p}}$ & $f_{n}$ & $a_{3 \mathrm{n}}$ & $a_{3 \mathrm{p} \text {-eff }}$ & $a_{3 \mathrm{n} \text {-eff }}$ \\
\hline${ }^{3} \mathrm{He}$ & 3.00 & 8.17 & 1.00 & 8.17 & 5.45 & 0.0 \\
${ }^{4} \mathrm{He}$ & 2.00 & 19.22 & 1.00 & 19.22 & 9.61 & 9.61 \\
${ }^{6} \mathrm{He}$ & 5.70 & 31.48 & 0.20 & 6.30 & 10.49 & 6.30 \\
${ }^{8} \mathrm{He}$ & 12.00 & 52.92 & 0.08 & 4.23 & 13.23 & 5.29 \\
${ }^{6} \mathrm{Li}$ & 1.50 & 11.34 & 1.00 & 11.34 & 7.56 & 7.56 \\
${ }^{7} \mathrm{Li}$ & 2.25 & 16.65 & 1.00 & 16.65 & 9.51 & 14.27 \\
${ }^{8} \mathrm{Li}$ & 3.16 & 21.36 & 0.75 & 16.02 & 10.68 & 16.02 \\
${ }^{9} \mathrm{Li}$ & 4.30 & 30.20 & 0.35 & 10.57 & 13.42 & 11.74 \\
${ }^{8} \mathrm{Be}$ & 1.15 & 13.29 & 1.00 & 13.29 & 9.97 & 9.97 \\
${ }^{9} \mathrm{Be}$ & 1.60 & 15.38 & 0.70 & 10.76 & 10.25 & 9.57 \\
${ }^{10} \mathrm{Be}$ & 2.20 & 18.50 & 0.70 & 12.95 & 11.10 & 12.95 \\
${ }^{10} \mathrm{~B}$ & 1.25 & 11.25 & 1.00 & 11.25 & 9.00 & 9.00 \\
${ }^{11} \mathrm{~B}$ & 3.50 & 35.84 & 0.85 & 30.46 & 26.07 & 27.69 \\
${ }^{12} \mathrm{C}$ & 1.20 & 21.68 & 1.00 & 21.68 & 18.06 & 18.06 \\
${ }^{16} \mathrm{O}$ & 1.00 & 17.64 & 1.00 & 17.64 & 15.43 & 15.43 \\
${ }^{27} \mathrm{Al}$ & 1.00 & 20.25 & 1.00 & 20.25 & 18.00 & 19.50 \\
${ }^{40} \mathrm{Ca}$ & 1.00 & 19.36 & 1.00 & 19.36 & 18.39 & 18.39 \\
${ }^{56} \mathrm{Fe}$ & 1.00 & 24.50 & 1.00 & 24.50 & 21.88 & 25.38 \\
${ }^{64} \mathrm{Cu}$ & 1.00 & 25.20 & 1.00 & 25.20 & 22.05 & 26.77 \\
${ }^{197} \mathrm{Au}$ & 1.00 & 20.79 & 1.00 & 20.79 & 16.47 & 24.70 \\
${ }^{208} \mathrm{~Pb}$ & 1.00 & 23.04 & 1.00 & 23.04 & 17.94 & 27.69 \\
\hline
\end{tabular}

The values of the proportionality factors $\mathrm{f}_{n}$ in the Table 4.2 are within the expected range $.85 \leq f_{n} \leq 1.15$. However, the values of the proportionality factors $\mathrm{f}_{p}$ are outside the expected range for some light nuclei.

The predictions for the SRC momentum distribution for proton and neutron for light nuclei $(\mathrm{A} \leq 12)$, calculated with Eqs. (4.1) and with the SRC parameters given in Tables 4.1 and 4.2, are compared with the momentum distributions from ab initio quantum Monte Carlo calculations (QMC) [69], the comparison is shown in Figs. $4.10,4.11$, and 4.12 for ${ }^{3} \mathrm{He},{ }^{8} \mathrm{He}$, and ${ }^{9}$ Be respectively. A good agreement, up to $\pm 15 \%$, is found between the predictions and the QMC calculations for momenta in the range $450-1000 \mathrm{MeV} / \mathrm{c}$, both for proton and neutron. The results show that the SRC high momentum nuclear spectral function models developed in the present dissertation, describe reasonably well the high momentum spectral function and the high momentum distributions for light nuclei in the nonrelativistic limit of the VN approximation. 


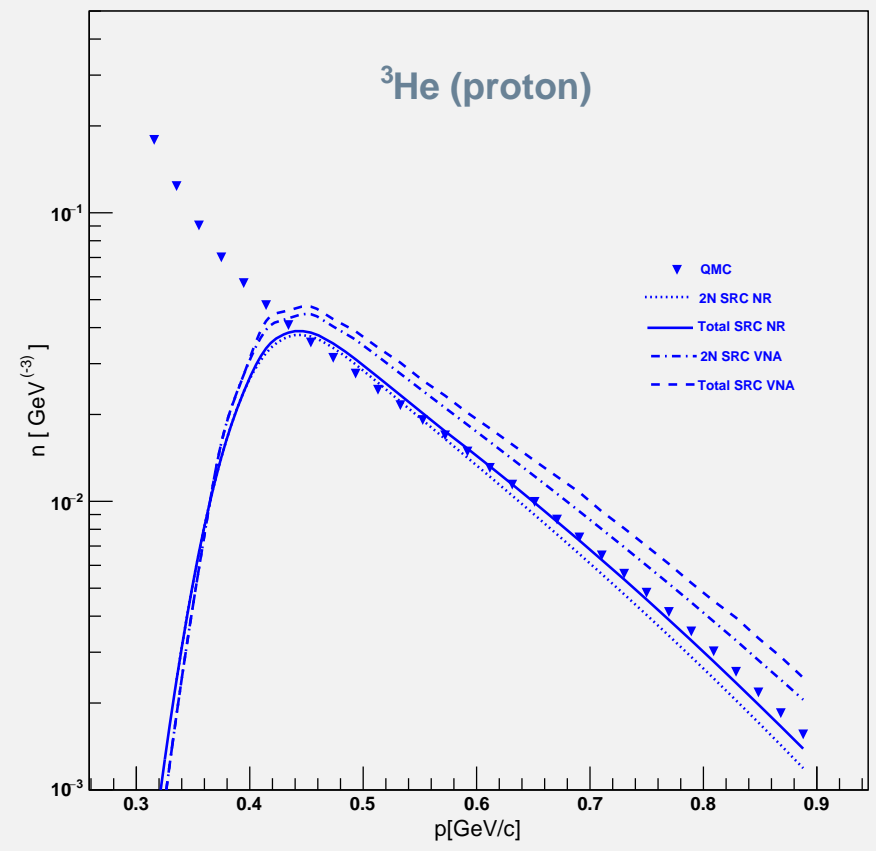

Figure 4.10: SRC momentum distribution for proton in ${ }^{3} \mathrm{He}$, in VNA and in the nonrelativistic limit of the VNA, the predictions in the nonrelativistic limit of the VNA are compared to QMC calculations

The values of momentum distribution for proton in ${ }^{8} \mathrm{He}$ are higher that for neutron in Fig.4.11. This result is expected since the relative fraction factor for proton, $x_{p}$, is smaller than the same factor for neutron, $x_{n}$, in a neutron-rich nucleus as ${ }^{8} \mathrm{He}$. Thus, since there are more neutrons than protons, the probability for a proton to be in SRC to a neutron is much higher than the probability for a neutron to be in SRC to a proton. 


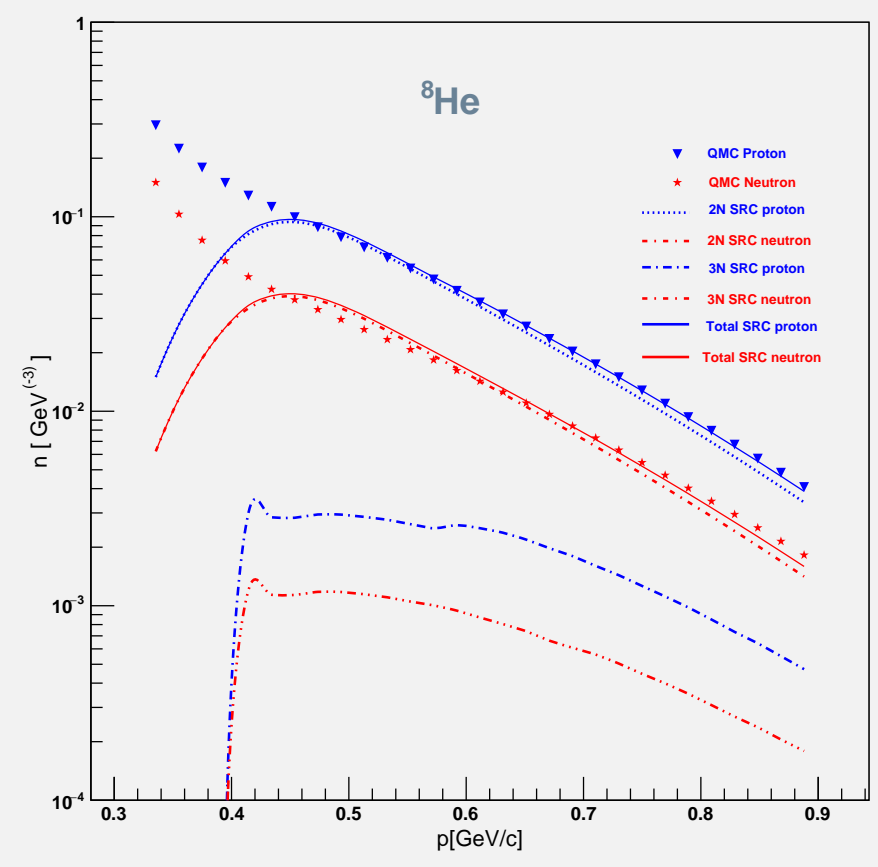

Figure 4.11: SRC momentum distribution for ${ }^{8} \mathrm{He}$ in the nonrelativistic limit of the VNA compared to QMC calculations

The nonrelativistic predictions of momentum distribution, calculated with Eqs. (4.1) and with the SRC parameters given in the Tables 4.1 and 4.2, for ${ }^{56} \mathrm{Fe}$ is depicted in the Fig. 4.13. The predictions are compared to the 2N SRC momentum distribution Ciofi/Simula results for proton given in [52]. The predictions have the same slope and greater values as compared to the Ciofi/Simula results for proton. The result is explained by considering that the predictions include the 3N SRC contributions as well as the corrections represented by the proton and neutron relative fraction factor $x_{p / n}$ (3.2), contributions and corrections that are not present in the Ciofi/Simula results. 


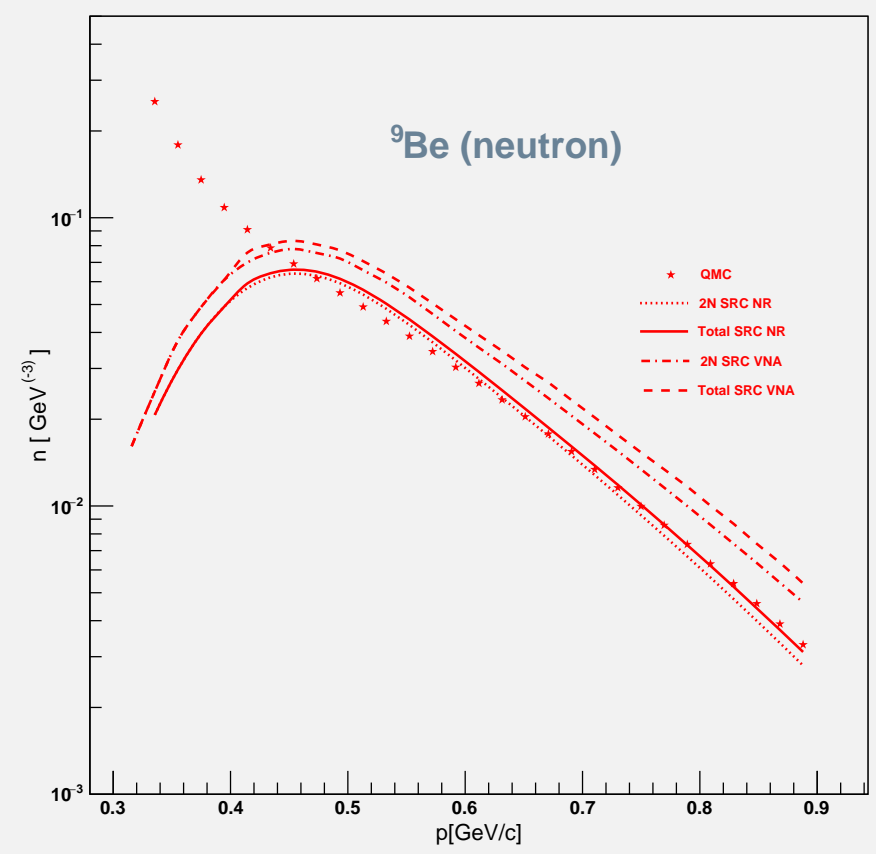

Figure 4.12: SRC momentum distribution for neutron in ${ }^{9} \mathrm{Be}$, in VNA and in the nonrelativistic limit of the VNA, the predictions in the nonrelativistic limit of the VNA are compared to QMC calculations

The predictions for the SRC momentum distribution for proton and neutron in the VN approximation are compared to the momentum distribution in the nonrelativistic limit of the VNA for ${ }^{208} \mathrm{~Pb}$. The results are calculated with the Eqs. (4.1) and with the SRC parameters given in the Tables 4.1 and 4.2. The VNA momentum distributions are as expected between $30 \%$ and $80 \%$ greater than the momentum distributions for the non relativistic case, the difference being greater for higher momentum. Similar results are obtained for the momentum distribution for proton in ${ }^{3} \mathrm{He}$ (Fig. 4.10), and for neutron in ${ }^{9} \mathrm{Be}$ (Fig. 4.12). 


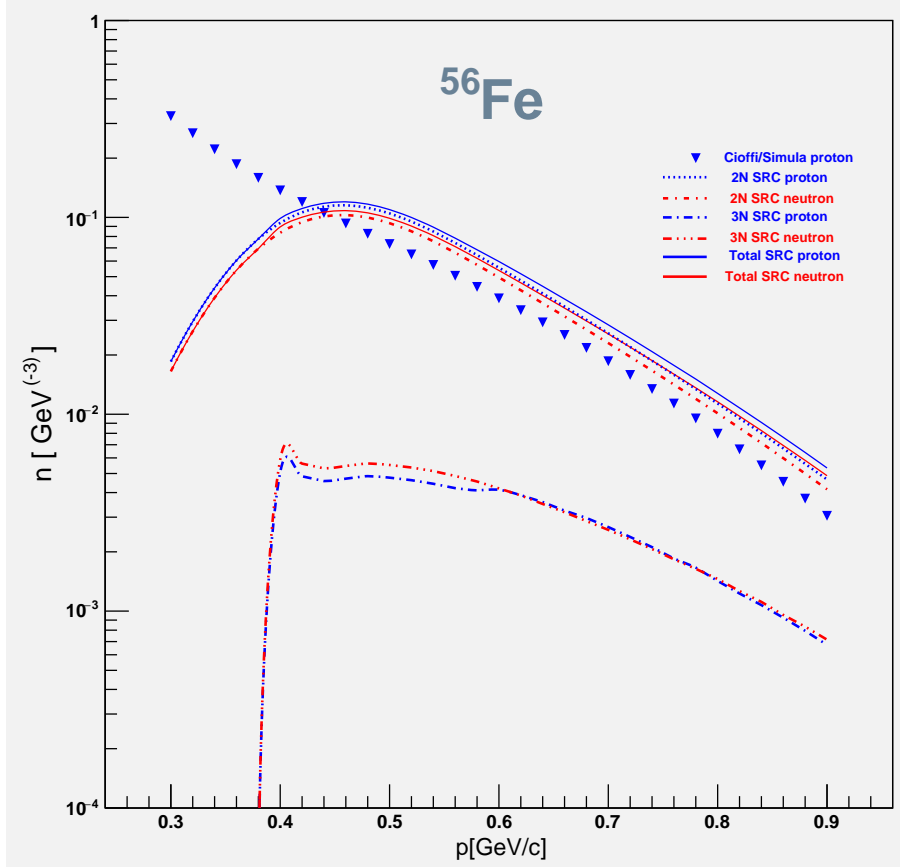

Figure 4.13: SRC momentum distribution for ${ }^{56} \mathrm{Fe}$ in the nonrelativistic limit of the VNA compared to 2N SRC momentum distribution for proton in Ciofi/Simula [52]

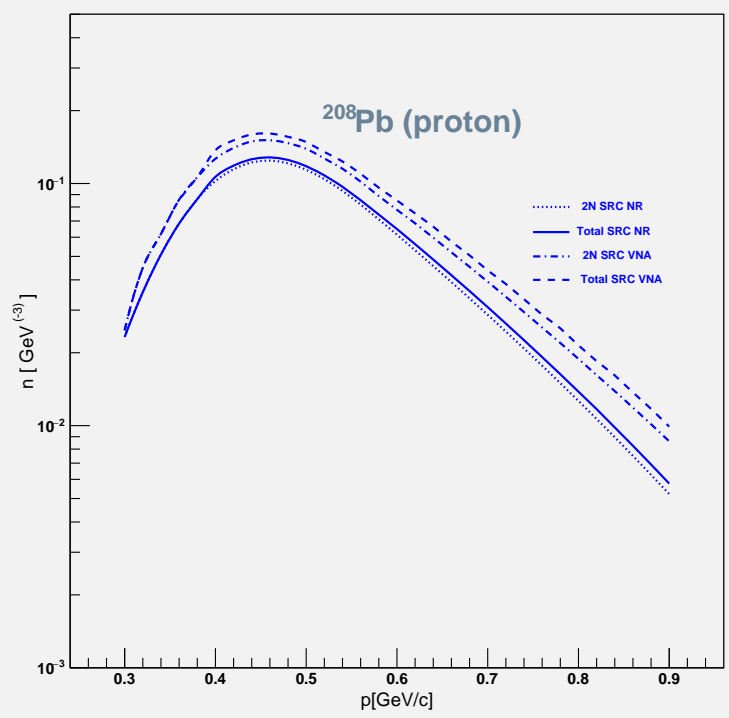

Figure 4.14: SRC momentum distribution for ${ }^{208} \mathrm{~Pb}$ in $\mathrm{VN}$ approximation compared to predictions in the nonrelativistic limit of the VNA 


\subsection{Parametrization of SRC and mean field momentum distribution}

The parametrization results for the mean field and the SRC momentum distributions are given in the present section. The parametrization of the nucleon momentum distribution for light $(\mathrm{A} \leq 12)$ and heavy nuclei $(16 \leq \mathrm{A} \leq 208)$ are obtained for the mean field momenta in the range $\left(0 \leq \mathrm{p} \leq 1.5 \mathrm{fm}^{-1}\right)$ and for the SRC momenta in the range $\left(1.5 \leq \mathrm{p} \leq 4.5 \mathrm{fm}^{-1}\right)$.

The nonrelativistic mean field momentum distribution $\mathrm{n}_{M F}$ for each nucleus is calculated with the following expressions

$$
\mathrm{n}_{\mathrm{MF}}(\mathrm{p})=\frac{1-4 \pi \int_{0}^{1} \mathrm{p}^{2}\left[\mathrm{n}_{\mathrm{A}, 2 \mathrm{~N}}^{\mathrm{p} / \mathrm{n}}(\mathrm{p})+\mathrm{n}_{\mathrm{A}, 3 \mathrm{~N}}^{\mathrm{p} / \mathrm{n}}(\mathrm{p})\right] \mathrm{dp}}{4 \pi \int_{0}^{1} \mathrm{k}^{2} \mathrm{n}_{0}(\mathrm{p})} \mathrm{n}_{0}(\mathrm{p})
$$

so that the total (MF plus SRC) momentum distribution, $\mathrm{n}_{\text {total }}$, is normalized to unity, namely

$$
4 \pi \int \mathrm{p}^{2} \mathrm{n}_{\text {total }}(\mathrm{p}) \mathrm{dp}=4 \pi \int_{0}^{1} \mathrm{p}^{2}\left[\mathrm{n}_{\mathrm{MF}}(\mathrm{p})+\mathrm{n}_{\mathrm{A}, 2 \mathrm{~N}}^{\mathrm{p} / \mathrm{n}}(\mathrm{p})+\mathrm{n}_{\mathrm{A}, 3 \mathrm{~N}}^{\mathrm{p} / \mathrm{n}}(\mathrm{p})\right] \mathrm{d} \mathrm{p}=1.0
$$

The SRC momentum distributions $n_{A, 2 N}^{p / n}$ and $n_{A, 3 N}^{p / n}$ are calculated by Eq.(4.1), with the SRC nuclear spectral functions in the VN approximation and VN approximation in the nonrelativistic limit. The mean field momentum distributions $\mathrm{n}_{0}$ for light nuclei are obtained from the QMC results given in Ref. [69]; for ${ }^{16} \mathrm{O}$ and ${ }^{40} \mathrm{Ca}$ from reference [52]; and for ${ }^{27} \mathrm{Al},{ }^{56} \mathrm{Fe}$, and ${ }^{208} \mathrm{~Pb}$ from the mean field wave function given for Zverev [123]

The equations for the parametrization of the mean field and the SRC momentum distributions are those given by Ciofi and Simula in Ref. [52]. The parametrization of the mean-field momentum distribution, in the range $0.0 \leq \mathrm{p} \leq 1.50 \mathrm{fm}^{-1}$ for light nuclei $(\mathrm{A} \leq 12)$, are obtained with the following equations

$$
n_{M F}(\mathrm{p})=\frac{A_{1}^{(0)} \frac{e^{-B_{1}^{(0)} \mathrm{p}^{2}}}{\left(1+C_{1}^{(0)} \mathrm{p}^{2}\right)^{2}}+A_{2}^{(0)} \frac{e^{-B_{2}^{(0)} \mathrm{p}^{2}}}{\left(1+C_{2}^{(0)} \mathrm{p}^{2}\right)^{2}}}{4 \pi}
$$


and for heavy nuclei $(16 \leq \mathrm{A} \leq 208)$ with

$$
n_{M F}(\mathrm{p})=\frac{A^{(0)} e^{-B^{(0)} \mathrm{p}^{2}}\left(1+C^{(0)} \mathrm{p}^{2}+D^{(0)} \mathrm{p}^{4}+E^{(0)} \mathrm{p}^{6}+F^{(0)} \mathrm{p}^{8}\right)}{4 \pi},
$$

where $4 \pi \int \mathrm{dpp}^{2} n_{M F}(\mathrm{p})=1$. For the parametrization of the SRC momentum distributions, in the range $1.5 \leq \mathrm{p} \leq 4.50 \mathrm{fm}^{-1}$, the equations are

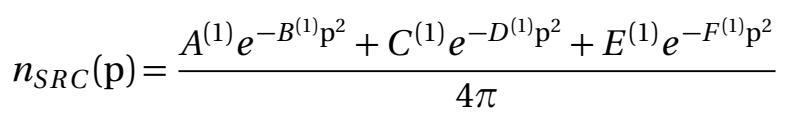

where $4 \pi \int \mathrm{dpp}^{2} n_{S R C}(\mathrm{p})=1$

\subsubsection{Parametrization of the SRC and the mean field momentum distribu- tion for the VN approximation in the nonrelativistic limit}

The parametrization of the momentum distribution for nuclei with $3 \leq \mathrm{A} \leq 208$ calculated with the nuclear spectral functions in the nonrelativistic limit of the VN approximation is obtained with Eqs. (4.5) to ( 4.7) for the mean-field and the SRC momentum distributions respectively. The corresponding parameters for the mean field and the SRC momentum distributions are given in Tables 4.3 to 4.6 , for light and heavy nuclei respectively. 
Table 4.3: Parameters for the non-relativistic mean-field momentum distribution for light nuclei A $\leq 12$

\begin{tabular}{|c||c|c|c|c|c|c|}
\hline Nucleus & $A_{1}^{(0)}\left(\mathrm{fm}^{3}\right)$ & $B_{1}^{(0)}\left(\mathrm{fm}^{2}\right)$ & $C_{1}^{(0)}\left(\mathrm{fm}^{2}\right)$ & $A_{2}^{(0)}\left(\mathrm{fm}^{3}\right)$ & $B_{2}^{(0)}\left(\mathrm{fm}^{2}\right)$ & $C_{2}^{(0)}\left(\mathrm{fm}^{2}\right)$ \\
\hline${ }^{3} \mathrm{He}(\mathrm{p})$ & 36.20 & 1.07 & 5.09 & 0.36 & 5.22 & 0.17 \\
${ }^{3} \mathrm{He}(\mathrm{n})$ & 21.60 & 0.49 & 5.13 & 5.54 & 4.80 & \\
${ }^{4} \mathrm{He}$ & 9.78 & 1.04 & 1.38 & 0.67 & 5.20 & \\
${ }^{6} \mathrm{He}(\mathrm{p})$ & 3.06 & 4.50 & 0.68 & 8.60 & 1.14 & 1.23 \\
${ }^{6} \mathrm{He}(\mathrm{n})$ & 27.68 & 1.19 & 3.29 & -20.91 & 7.08 & 6.50 \\
${ }^{8} \mathrm{He}(\mathrm{p})$ & 12.14 & 0.99 & 1.72 & -1.80 & 8.65 & \\
${ }^{8} \mathrm{He}(\mathrm{n})$ & 16.45 & 1.78 & 1.21 & -13.58 & 7.79 & 3.07 \\
${ }^{6} \mathrm{Li}$ & 14.04 & 1.34 & 1.20 & -4.30 & 13.20 & \\
${ }^{7} \mathrm{Li}(\mathrm{p})$ & 11.11 & 1.33 & 1.13 & -2.70 & 8.58 & \\
${ }^{7} \mathrm{Li}(\mathrm{n})$ & 10.08 & 1.60 & 0.71 & -3.59 & 9.45 & \\
${ }^{8} \mathrm{Li}(\mathrm{p})$ & 8.17 & 1.72 & 0.46 & -1.55 & 8.37 & \\
${ }^{8} \mathrm{Li}(\mathrm{n})$ & 14.17 & 1.23 & 1.37 & -10.48 & 7.97 & \\
${ }^{9} \mathrm{Li}(\mathrm{p})$ & 7.88 & 1.34 & 0.70 & -1.80 & 7.65 & \\
${ }^{9} \mathrm{Li}(\mathrm{n})$ & 7.68 & 2.09 & 0.094 & -4.92 & 10.82 & \\
${ }^{8} \mathrm{Be}(\mathrm{p})$ & 7.98 & 1.34 & 0.70 & -1.64 & 7.50 & \\
${ }^{9} \mathrm{Be}(\mathrm{p})$ & 8.17 & 1.44 & 0.63 & -1.92 & 6.57 & \\
${ }^{9} \mathrm{Be}(\mathrm{n})$ & 7.99 & 1.89 & 0.27 & -3.21 & 8.64 & \\
${ }^{10} \mathrm{Be}(\mathrm{p})$ & 7.08 & 2.32 & -0.026 & -2.02 & 10.37 & \\
${ }^{10} \mathrm{Be}(\mathrm{n})$ & 8.36 & 2.01 & 0.20 & -5.41 & 8.89 & \\
${ }^{12} \mathrm{C}$ & 6.70 & 1.26 & 0.45 & -3.41 & 4.90 & \\
\hline
\end{tabular}

Table 4.4: Parameters for the non-relativistic mean-field momentum distribution for heavy nuclei $16 \leq \mathrm{A} \leq 208$

\begin{tabular}{|c||c|c|c|c|c|c|}
\hline Nucleus & $A^{(0)}\left(\mathrm{fm}^{3}\right)$ & $B^{(0)}\left(\mathrm{fm}^{2}\right)$ & $C^{(0)}\left(\mathrm{fm}^{2}\right)$ & $D^{(0)}\left(\mathrm{fm}^{4}\right)$ & $E^{(0)}\left(\mathrm{fm}^{6}\right)$ & $F^{(0)}\left(\mathrm{fm}^{8}\right)$ \\
\hline${ }^{16} \mathrm{O}(\mathrm{p})$ & 2.93 & 3.01 & 6.60 & & & \\
${ }^{27} \mathrm{Al}(\mathrm{p})$ & 1.83 & 3.10 & 7.00 & 3.51 & & \\
${ }^{27} \mathrm{Al}(\mathrm{n})$ & 2.30 & 3.24 & 3.75 & 6.18 & & \\
${ }^{40} \mathrm{Ca}(\mathrm{p})$ & 3.24 & 3.60 & 0.00 & 11.10 & & \\
${ }^{56} \mathrm{Fe} \mathrm{(p)}$ & 3.03 & 3.84 & -0.17 & 11.42 & 3.63 & \\
${ }^{56} \mathrm{Fe} \mathrm{(n)}$ & 2.65 & 3.80 & 2.60 & 1.89 & 12.18 & \\
${ }^{208} \mathrm{~Pb}(\mathrm{p})$ & 2.59 & 5.55 & 1.08 & 49.22 & -121.16 & 210.69 \\
${ }^{208} \mathrm{~Pb}(\mathrm{n})$ & 1.89 & 3.61 & 5.66 & -9.36 & 24.30 & \\
\hline
\end{tabular}

The momentum distributions calculated with the SRC nuclear spectral functions in the nonrelativistic limit of the VN approximation, compared to the curve fitting obtained with the parameters in Table 4.3 to Table 4.6, are presented in Figs. 4.15 and 4.16, for some light and heavy nuclei respectively. The agreement between the curve fitted with the parameters and 
those from numerical calculations is, in general, good for all the range of momentum considered.
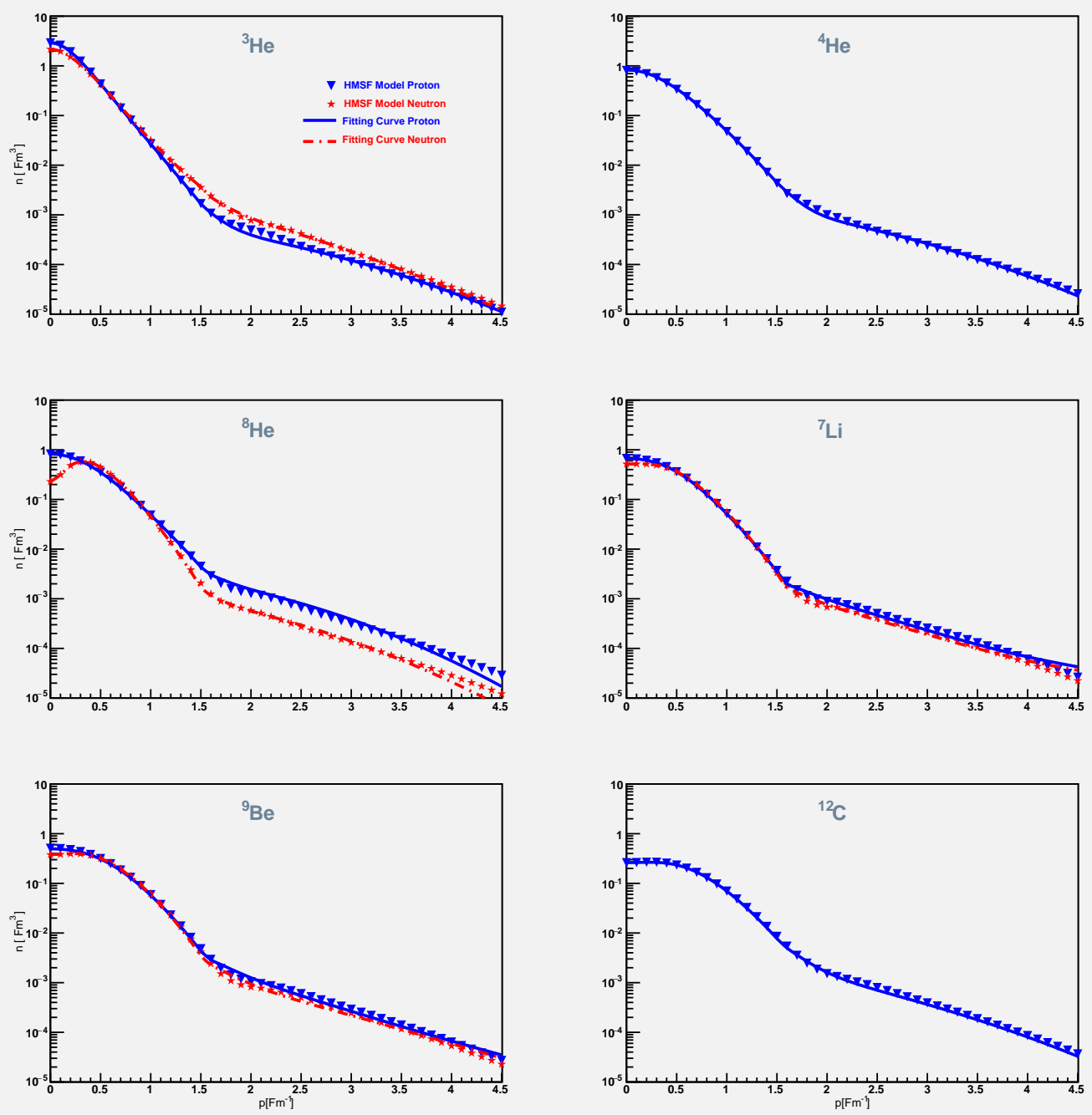

Figure 4.15: Momentum distribution for light nuclei calculated with the SRC nuclear spectral functions in the nonrelativistic limit of the VNA, compared to the curve fitting obtained with the mean field parameters in Table 4.3 and SRC parameters in Table 4.5 

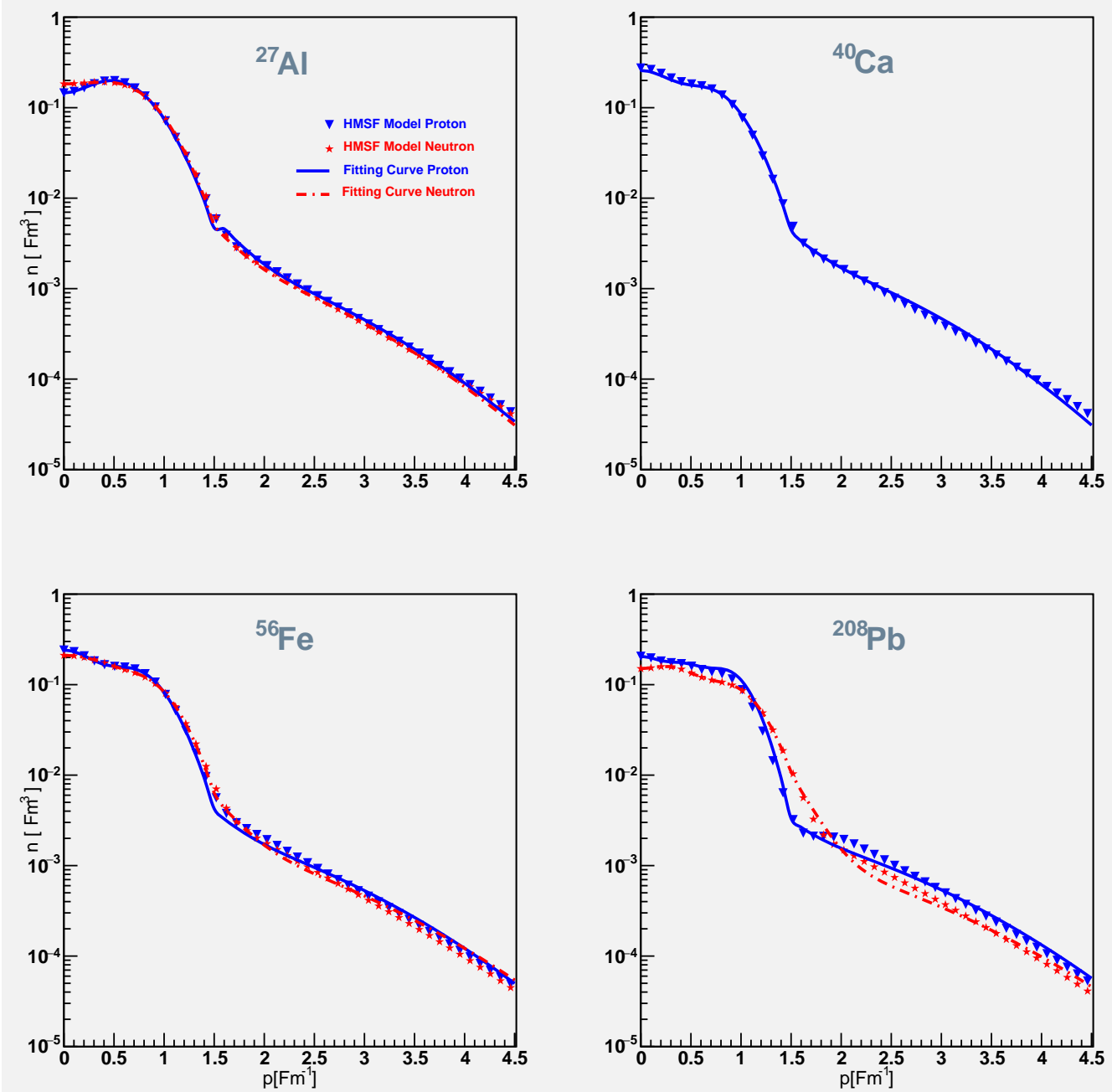

Figure 4.16: Momentum distribution for heavy nuclei calculated with the SRC nuclear spectral functions in the nonrelativistic limit of the VNA, compared to the curve fitting obtained with the mean field parameters in Table 4.4 and SRC parameters in Table 4.6 
Table 4.5: Parameters for the SRC momentum distribution in the nonrelativistic limit of the VNA for light nuclei $\mathrm{A} \leq 12$

\begin{tabular}{|c||c|c|c|c|c|c|}
\hline Nucleus & $A^{(1)}\left(\mathrm{fm}^{3}\right)$ & $B^{(1)}\left(\mathrm{fm}^{2}\right)$ & $C^{(1)}\left(\mathrm{fm}^{3}\right)$ & $D^{(1)}\left(\mathrm{fm}^{2}\right)$ & $E^{(1)}\left(\mathrm{fm}^{3}\right)$ & $F^{(1)}\left(\mathrm{fm}^{2}\right)$ \\
\hline${ }^{3} \mathrm{He}(\mathrm{p})$ & 0.93 & 1.87 & 0.009 & 0.22 & 0.0014 & 0.18 \\
${ }^{3} \mathrm{He}(\mathrm{n})$ & 0.94 & 1.64 & 0.026 & 0.34 & 0.006 & 0.19 \\
${ }^{4} \mathrm{He}$ & 3.16 & 1.92 & 0.021 & 0.22 & 0.0023 & 0.19 \\
${ }^{6} \mathrm{He}(\mathrm{p})$ & 0.35 & 0.95 & 0.015 & 0.19 & & \\
${ }^{6} \mathrm{He}(\mathrm{n})$ & 0.60 & 1.60 & 0.0114 & 0.20 & & \\
${ }^{8} \mathrm{He}(\mathrm{p})$ & 0.87 & 1.67 & -0.040 & 0.34 & 0.091 & 0.29 \\
${ }^{8} \mathrm{He}(\mathrm{n})$ & 0.50 & 1.83 & 0.02 & 0.27 & & \\
${ }^{6} \mathrm{Li}(\mathrm{p})$ & 3.00 & 1.90 & 0.02 & 0.22 & 0.001 & 0.30 \\
${ }^{7} \mathrm{LI}(\mathrm{p})$ & 0.20 & 1.21 & 0.033 & 0.32 & 0.002 & 0.07 \\
${ }^{7} \mathrm{Li}(\mathrm{n})$ & 0.26 & 1.32 & 0.024 & 0.30 & 0.0013 & 0.058 \\
${ }^{8} \mathrm{LI}(\mathrm{p})$ & 0.24 & 1.12 & 0.041 & 0.30 & 0.0013 & 0.072 \\
${ }^{8} \mathrm{Li}(\mathrm{n})$ & 0.27 & 1.18 & 0.021 & 0.27 & 0.001 & 0.09 \\
${ }^{9} \mathrm{LI}(\mathrm{p})$ & 0.26 & 1.10 & 0.041 & 0.30 & 0.0017 & 0.07 \\
${ }^{9} \mathrm{Li} \mathrm{(n)}$ & 0.25 & 1.20 & 0.02 & 0.27 & 0.0012 & 0.08 \\
${ }^{8} \mathrm{Be}$ & 0.36 & 1.07 & 0.015 & 0.22 & 0.0052 & 0.16 \\
${ }^{9} \mathrm{Be}(\mathrm{p})$ & 0.31 & 1.11 & 0.035 & 0.33 & 0.0045 & 0.12 \\
${ }^{9} \mathrm{Be}(\mathrm{n})$ & 0.29 & 1.12 & 0.019 & 0.30 & 0.0047 & 0.13 \\
${ }^{10} \mathrm{Be}(\mathrm{p})$ & 0.31 & 1.11 & 0.035 & 0.33 & 0.0045 & 0.12 \\
${ }^{10} \mathrm{Be}(\mathrm{n})$ & 0.28 & 1.13 & 0.018 & 0.31 & 0.0045 & 0.14 \\
${ }^{12} \mathrm{C}$ & 1.79 & 1.50 & 0.032 & 0.57 & 0.029 & 0.21 \\
\hline
\end{tabular}

Table 4.6: Parameters for the SRC momentum distribution in the nonrelativistic limit of the VNA for heavy nuclei $16 \leq \mathrm{A} \leq 208$

\begin{tabular}{|c||c|c|c|c|c|c|}
\hline Nucleus & $A^{(1)}\left(\mathrm{fm}^{3}\right)$ & $B^{(1)}\left(\mathrm{fm}^{2}\right)$ & $C^{(1)}\left(\mathrm{fm}^{3}\right)$ & $D^{(1)}\left(\mathrm{fm}^{2}\right)$ & $E^{(1)}\left(\mathrm{fm}^{3}\right)$ & $F^{(1)}\left(\mathrm{fm}^{2}\right)$ \\
\hline${ }^{16} \mathrm{O}(\mathrm{p})$ & 0.48 & 1.33 & 0.057 & 0.25 & -0.011 & 0.35 \\
${ }^{27} \mathrm{Al}(\mathrm{p})$ & 0.80 & 1.25 & 0.045 & 0.23 & & \\
${ }^{27} \mathrm{Al}(\mathrm{n})$ & 0.60 & 1.25 & 0.041 & 0.23 & & \\
${ }^{40} \mathrm{Ca}(\mathrm{p})$ & 0.45 & 1.31 & 0.058 & 0.246 & -0.011 & 0.35 \\
${ }^{56} \mathrm{Fe}(\mathrm{p})$ & 0.40 & 1.27 & 0.044 & 0.21 & & \\
${ }^{56} \mathrm{Fe} \mathrm{(n)}$ & 0.75 & 1.20 & 0.032 & 0.19 & & \\
${ }^{208} \mathrm{~Pb}(\mathrm{p})$ & 0.36 & 1.45 & 0.041 & 0.20 & & \\
${ }^{208} \mathrm{~Pb}(\mathrm{n})$ & 3.12 & 1.48 & 0.0022 & 0.18 & & \\
\hline
\end{tabular}

\subsubsection{The parametrization of the SRC and the mean field momentum distri- bution in virtual nucleon approximation}

The parametrization of the momentum distribution for nuclei with $3 \leq \mathrm{A} \leq 208$ calculated with the nuclear spectral functions in the VN approximation is obtained with Eqs. (4.5) to ( 4.7) for 
the mean-field and the SRC momentum distributions respectively. The corresponding parameters for the mean field and the SRC momentum distributions are given in the Tables 4.7 to 4.10 , for light and heavy nuclei respectively.

Table 4.7: Parameters for the mean-field momentum distribution in VNA for light nuclei A $\leq 12$

\begin{tabular}{|c||c|c|c|c|c|c|}
\hline Nucleus & $A_{1}^{(0)}\left(\mathrm{fm}^{3}\right)$ & $B_{1}^{(0)}\left(\mathrm{fm}^{2}\right)$ & $C_{1}^{(0)}\left(\mathrm{fm}^{2}\right)$ & $A_{2}^{(0)}\left(\mathrm{fm}^{3}\right)$ & $B_{2}^{(0)}\left(\mathrm{fm}^{2}\right)$ & $C_{2}^{(0)}\left(\mathrm{fm}^{2}\right)$ \\
\hline${ }^{3} \mathrm{He}(\mathrm{p})$ & 36.20 & 0.97 & 5.09 & 0.36 & 6.22 & 0.17 \\
${ }^{3} \mathrm{He}(\mathrm{n})$ & 21.60 & 0.49 & 5.13 & 5.54 & 4.80 & \\
${ }^{4} \mathrm{He}$ & 9.78 & 1.04 & 1.38 & 0.67 & 5.20 & \\
${ }^{6} \mathrm{He}(\mathrm{p})$ & 3.06 & 4.50 & 0.68 & 9.00 & 1.14 & 1.23 \\
${ }^{6} \mathrm{He}(\mathrm{n})$ & 27.68 & 1.19 & 3.15 & -20.91 & 7.08 & 6.50 \\
${ }^{8} \mathrm{He}(\mathrm{p})$ & 12.14 & 0.99 & 1.72 & -1.80 & 8.65 & \\
${ }^{8} \mathrm{He}(\mathrm{n})$ & 16.45 & 1.78 & 1.13 & -13.58 & 7.79 & 3.07 \\
${ }^{6} \mathrm{Li}$ & 13.40 & 1.34 & 1.20 & -4.30 & 13.20 & \\
${ }^{7} \mathrm{Li}(\mathrm{p})$ & 11.11 & 1.33 & 1.13 & -2.70 & 8.58 & \\
${ }^{7} \mathrm{Li}(\mathrm{n})$ & 10.08 & 1.60 & 0.71 & -3.59 & 9.45 & \\
${ }^{8} \mathrm{Li}(\mathrm{p})$ & 8.17 & 1.62 & 0.46 & -1.55 & 8.37 & \\
${ }^{8} \mathrm{Li}(\mathrm{n})$ & 14.17 & 1.23 & 1.37 & -10.48 & 7.97 & \\
${ }^{9} \mathrm{Li}(\mathrm{p})$ & 7.88 & 1.34 & 0.70 & -1.80 & 7.65 & \\
${ }^{9} \mathrm{Li}(\mathrm{n})$ & 7.68 & 2.09 & 0.094 & -4.92 & 10.82 & \\
${ }^{8} \mathrm{Be}(\mathrm{p})$ & 7.98 & 1.34 & 0.70 & -1.64 & 7.50 & \\
${ }^{9} \mathrm{Be}(\mathrm{p})$ & 8.17 & 1.44 & 0.63 & -1.92 & 6.57 & \\
${ }^{9} \mathrm{Be}(\mathrm{n})$ & 7.99 & 1.89 & 0.27 & -3.21 & 8.64 & \\
${ }^{10} \mathrm{Be}(\mathrm{p})$ & 7.08 & 2.32 & -0.026 & -2.02 & 10.37 & \\
${ }^{10} \mathrm{Be}(\mathrm{n})$ & 8.36 & 2.01 & 0.20 & -5.41 & 8.89 & \\
${ }^{12} \mathrm{C}$ & 6.70 & 1.26 & 0.45 & -3.41 & 4.90 & \\
\hline
\end{tabular}

Table 4.8: Parameters for the mean-field momentum distribution in VNA for heavy nuclei $16 \leq$ A $\leq 208$

\begin{tabular}{|c||c|c|c|c|c|c|}
\hline Nucleus & $A^{(0)}\left(\mathrm{fm}^{3}\right)$ & $B^{(0)}\left(\mathrm{fm}^{2}\right)$ & $C^{(0)}\left(\mathrm{fm}^{2}\right)$ & $D^{(0)}\left(\mathrm{fm}^{4}\right)$ & $E^{(0)}\left(\mathrm{fm}^{6}\right)$ & $F^{(0)}\left(\mathrm{fm}^{8}\right)$ \\
\hline${ }^{16} \mathrm{O}(\mathrm{p})$ & 2.73 & 3.00 & 6.60 & & & \\
${ }^{27} \mathrm{Al}(\mathrm{p})$ & 1.65 & 3.10 & 7.00 & 6.00 & & \\
${ }^{27} \mathrm{Al}(\mathrm{n})$ & 2.30 & 3.24 & 3.75 & 7.18 & & \\
${ }^{40} \mathrm{Ca}(\mathrm{p})$ & 3.27 & 3.55 & 0.07 & 10.41 & & \\
${ }^{56} \mathrm{Fe}(\mathrm{p})$ & 2.66 & 3.85 & -0.20 & 11.46 & 6.99 & \\
${ }^{56} \mathrm{Fe}(\mathrm{n})$ & 2.50 & 3.81 & 2.55 & 2.05 & 12.20 & \\
${ }^{208} \mathrm{~Pb}(\mathrm{p})$ & 2.15 & 5.20 & 1.16 & 44.33 & -120.00 & 180.96 \\
${ }^{208} \mathrm{~Pb}(\mathrm{n})$ & 1.76 & 3.59 & 5.61 & -9.47 & 24.12 & \\
\hline
\end{tabular}


Table 4.9: Parameters for the SRC momentum distribution in VNA for light nuclei A $\leq 12$

\begin{tabular}{|c||c|c|c|c|}
\hline Nucleus & $A^{(1)}\left(\mathrm{fm}^{3}\right)$ & $B^{(1)}\left(\mathrm{fm}^{2}\right)$ & $C^{(1)}\left(\mathrm{fm}^{3}\right)$ & $D^{(1)}\left(\mathrm{fm}^{2}\right)$ \\
\hline${ }^{3} \mathrm{He}(\mathrm{p})$ & 0.39 & 1.35 & 0.014 & 0.21 \\
${ }^{3} \mathrm{He}(\mathrm{n})$ & 0.10 & 0.59 & 0.015 & 0.19 \\
${ }^{4} \mathrm{He}$ & 0.20 & 0.83 & 0.027 & 0.20 \\
${ }^{6} \mathrm{He}(\mathrm{p})$ & 0.24 & 0.95 & 0.030 & 0.20 \\
${ }^{6} \mathrm{He}(\mathrm{n})$ & 0.17 & 1.10 & 0.020 & 0.21 \\
${ }^{8} \mathrm{He}(\mathrm{p})$ & 0.24 & 0.85 & 0.030 & 0.19 \\
${ }^{8} \mathrm{He} \mathrm{(n)}$ & 0.14 & 1.15 & 0.018 & 0.22 \\
${ }^{6} \mathrm{Li} \mathrm{(p)}$ & 0.19 & 0.83 & 0.027 & 0.20 \\
${ }^{7} \mathrm{LI}(\mathrm{p})$ & 0.19 & 0.90 & 0.028 & 0.20 \\
${ }^{7} \mathrm{Li} \mathrm{(n)}$ & 0.12 & 0.77 & 0.020 & 0.19 \\
${ }^{8} \mathrm{LI}(\mathrm{p})$ & 0.19 & 0.90 & 0.028 & 0.19 \\
${ }^{8} \mathrm{Li} \mathrm{(n)}$ & 0.14 & 0.73 & 0.020 & 0.19 \\
${ }^{9} \mathrm{LI}(\mathrm{p})$ & 0.21 & 0.77 & 0.028 & 0.19 \\
${ }^{9} \mathrm{Li} \mathrm{(n)}$ & 0.13 & 0.76 & 0.017 & 0.18 \\
${ }^{8} \mathrm{Be}$ & 0.21 & 0.80 & 0.030 & 0.20 \\
${ }^{9} \mathrm{Be} \mathrm{(p)}$ & 0.21 & 0.77 & 0.028 & 0.19 \\
${ }^{9} \mathrm{Be}(\mathrm{n})$ & 0.14 & 0.75 & 0.019 & 0.175 \\
${ }^{10} \mathrm{Be}(\mathrm{p})$ & 0.15 & 0.83 & 0.026 & 0.18 \\
${ }^{10} \mathrm{Be}(\mathrm{n})$ & 0.13 & 0.78 & 0.018 & 0.17 \\
${ }^{12} \mathrm{C}$ & 0.33 & 0.73 & 0.025 & 0.16 \\
\hline
\end{tabular}

Table 4.10: Parameters for the SRC momentum distribution in VNA for heavy nuclei $16 \leq \mathrm{A} \leq$ 208

\begin{tabular}{|c||c|c|c|c|c|c|}
\hline Nucleus & $A^{(1)}\left(\mathrm{fm}^{3}\right)$ & $B^{(1)}\left(\mathrm{fm}^{2}\right)$ & $C^{(1)}\left(\mathrm{fm}^{3}\right)$ & $D^{(1)}\left(\mathrm{fm}^{2}\right)$ & $E^{(1)}\left(\mathrm{fm}^{3}\right)$ & $F^{(1)}\left(\mathrm{fm}^{2}\right)$ \\
\hline${ }^{16} \mathrm{O}(\mathrm{p})$ & 0.20 & 0.96 & -0.028 & 0.22 & 0.069 & 0.21 \\
${ }^{27} \mathrm{Al}(\mathrm{p})$ & 0.52 & 1.11 & 0.047 & 0.20 & -0.035 & 0.89 \\
${ }^{27} \mathrm{Al}(\mathrm{n})$ & 0.52 & 1.11 & 0.043 & 0.20 & -0.020 & 0.73 \\
${ }^{40} \mathrm{Ca}(\mathrm{p})$ & 0.21 & 0.90 & -0.027 & 0.24 & 0.069 & 0.21 \\
${ }^{56} \mathrm{Fe} \mathrm{(p)}$ & 0.24 & 0.86 & -0.025 & 0.23 & 0.069 & 0.20 \\
${ }^{56} \mathrm{Fe} \mathrm{(n)}$ & 0.42 & 1.04 & 0.048 & 0.20 & -0.05 & 1.00 \\
${ }^{208} \mathrm{~Pb}(\mathrm{p})$ & 0.23 & 0.90 & -0.025 & 0.25 & 0.071 & 0.20 \\
${ }^{208} \mathrm{~Pb}(\mathrm{n})$ & 1.46 & 1.18 & 0.026 & 0.16 & & \\
\hline
\end{tabular}

The momentum distribution calculated with the SRC nuclear spectral functions in the VN approximation, compared to the curve fitting obtained with the parameters in Table 4.7 to Table 4.10, is presented in Figs. 4.17 and 4.18, for some light and heavy nuclei respectively. The agreement between the curve fitted with the parameters and those from numerical calculations is, in general, good for all the range of momentum considered. 

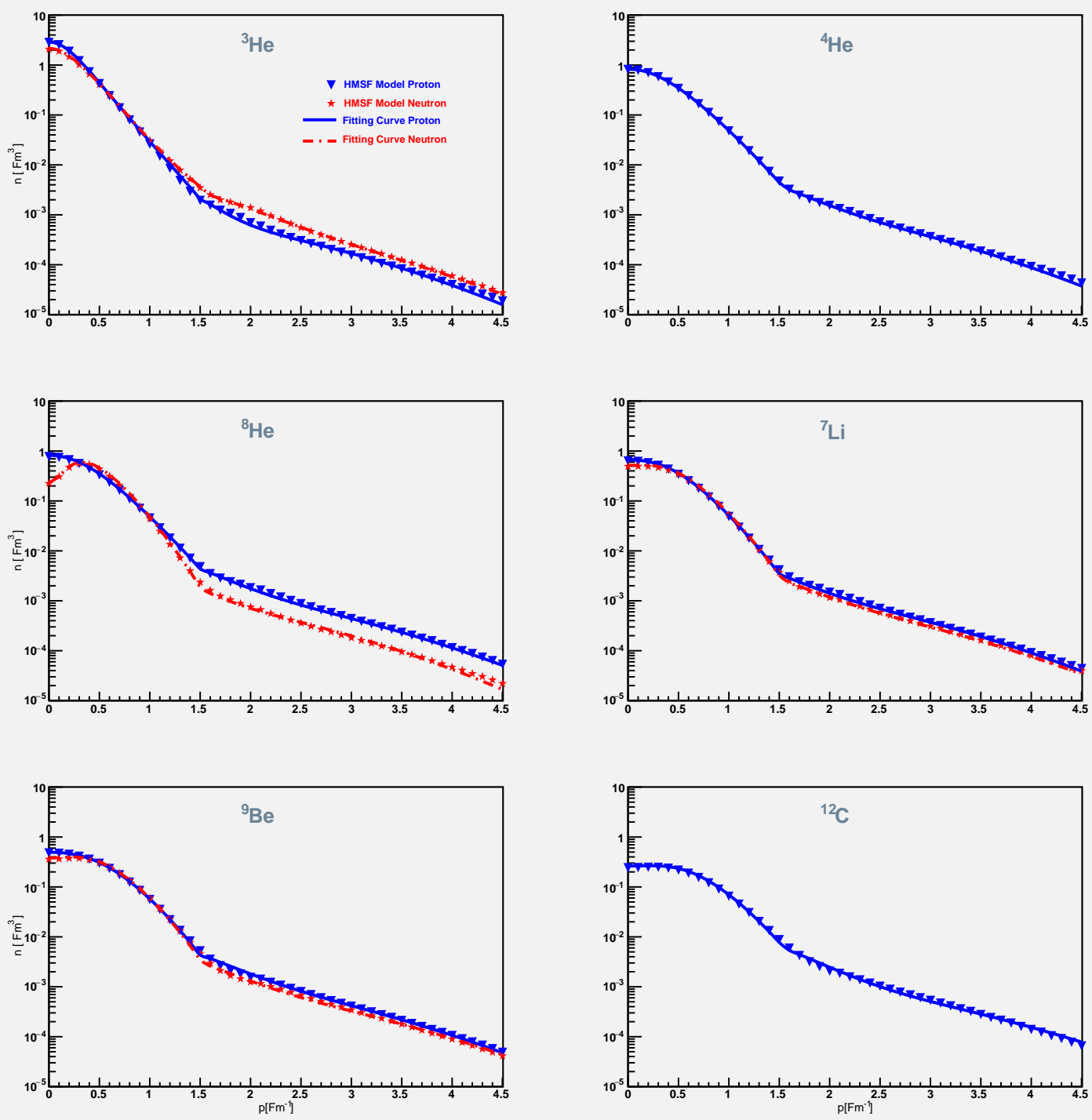

Figure 4.17: Momentum distribution for light nuclei calculated with the SRC nuclear spectral functions for VNA, compared to the curve fitting obtained with the mean field parameters in Table 4.7 and the SRC parameters in Table 4.9 

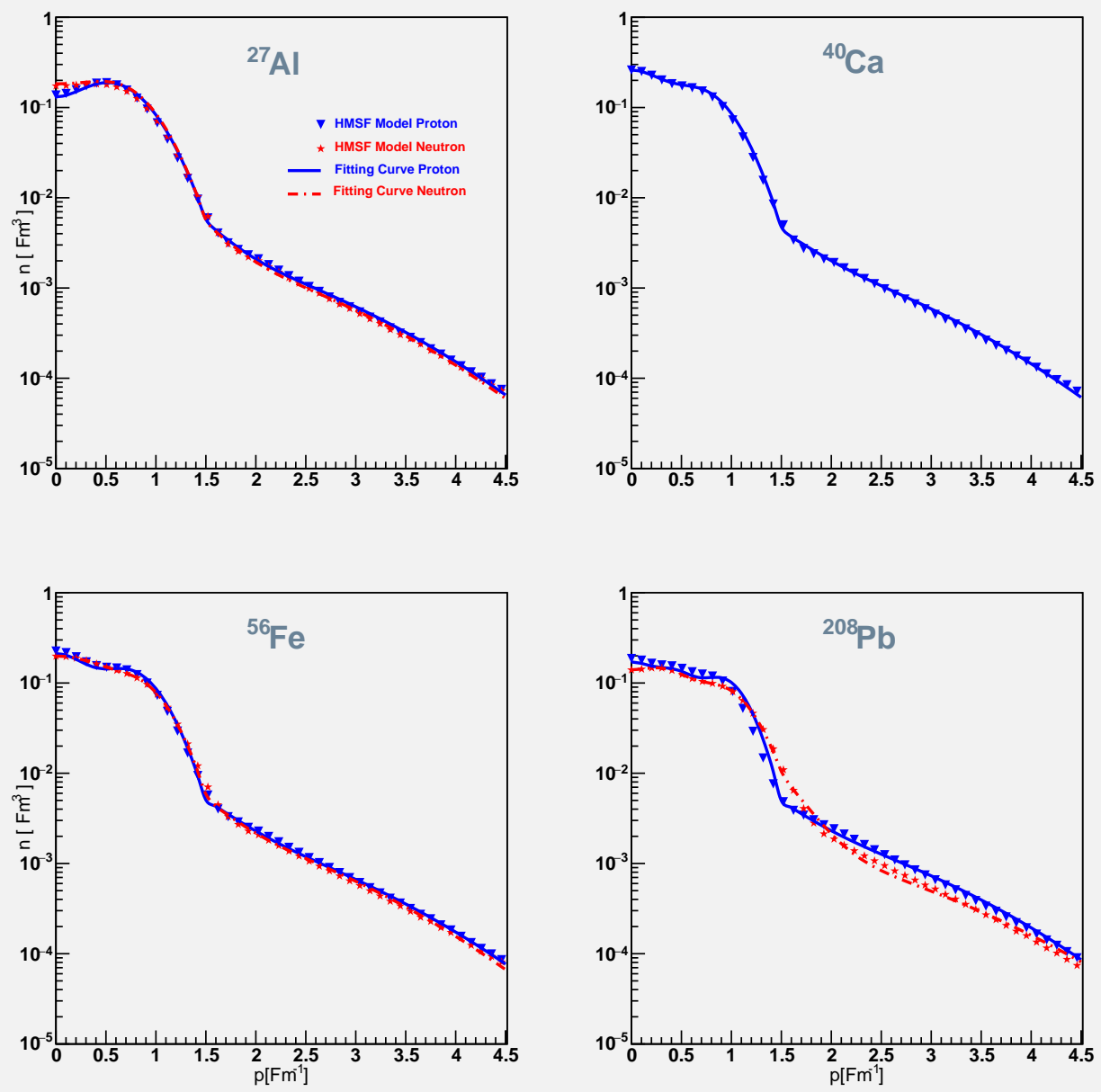

Figure 4.18: Momentum distribution for heavy nuclei calculated with the SRC nuclear spectral functions for VNA, compared to the curve fitting obtained with the mean field parameters in Table 4.8 and the SRC parameters in Table 4.10

\subsection{Summary}

The numerical estimates of the $2 \mathrm{~N}$ and $3 \mathrm{~N}$ SRC nuclear spectral functions in the LF and VN approximations, as well as the numerical estimates and the parametrization of momentum distribution of the bound nucleon, both for $\mathrm{VN}$ and $\mathrm{VN}$ in the nonrelativistic limit approximations, are obtained in the present chapter. The numerical estimates for the LF SRC density matrices are also calculated. The nuclear spectral functions and the LF SRC density matrices are calculated with the computational models developed in chapter 3. 
Before calculating the numerical estimates of the nuclear spectral functions, the range of validity and set of parameters used for the numerical estimates are briefly discussed.

The $\mathrm{D}$ and S-waves deuteron momentum distribution $\left(n_{d}\right)$ are defined as the result from a wave function modeled with the Argone V18 (AV18) two-nucleon potential, that is specially suitable for the spectral function model since fits both $p p$ and $n p$ data [51]. The numerical estimates for the deuteron LF density matrix are also included in the present chapter.

The numerical estimates for the $2 \mathrm{~N}$ and $3 \mathrm{~N}$ SRC momentum distribution for proton and neutron are calculated by integration of the nuclear spectral functions obtained in the computational models of chapter 3. These momentum distributions are compared for light nuclei (A $\leq 12$ ) with the $a b$ initio quantum Monte Carlo calculations given in [69], and for some heavy nuclei $(A>12)$ with the calculations for proton included in [52].

The mean-field and SRC momentum distributions in VNA and VN in the nonrelativistic limit are parametrized for a wide range of nuclei $(3 \leq \mathrm{A} \leq 208)$, resulting in a set of curve fitting parameters useful for further calculations. 


\section{CHAPTER 5}

\section{Conclusions}

For momenta below the Fermi momentum $\mathrm{k}_{F} \sim 250 \mathrm{MeV} / c$, the nuclear shell model, derived from the mean field picture of the nucleus, has been successful in correctly predicting all nuclear magic numbers, as well as in describing a large amount of nuclear data. The main assumption of the nuclear mean field model is that the nucleons are independent particles moving in an average or mean field generated by the remaining $(A-1)$ nucleons in the nucleus. As a result each nucleon is independent of the exact instantaneous position of all other nucleons. The nuclear shell model is valid for nucleons with long range ( $\geq 2 \mathrm{fm}$ ) mutual separations.

The nuclear shell model however is found to break down for inter nucleon distances smaller than $2 r_{N}$, where $r_{N} \approx 0.85 \mathrm{fm}$ is the radius of the nucleon, for which two nucleons start to overlap and the notion of the mean field become invalid. Their dynamics are mainly defined by the NN interaction at short distances which is dominated by the tensor interaction $(\sim 0.8-1.2 \mathrm{fm})$ and repulsive core $(\leq 0.5-0.7 \mathrm{fm})$. Such configurations are generally referred to as 2N Short Range Correlations (SRCs). The domain of multi-nucleon SRCs is characterized by relativistic momenta of the probed nucleon. Another high energy nuclear phenomena for which the nuclear shell model is not valid, is the modification of quark distribution of a bound nucleon in the nucleus, as compared to that of a free nucleon, the so called EMC effect.

In 2011, the strong correlation between the EMC effect and the 2N SRC in the nucleus was observed through high energy experiments (well above $\mathrm{k}_{F}$ ). Since the SRC structure in the nucleus implies high momentum bound nucleons, the experiments indicate that the EMC effect is only due to the high momentum component of the nuclear wave function, so that the possible modification of the parton distributions in nucleons in the nucleus occur only in nucleons belonging to SRCs.

A further understanding of the dynamics involved in the above described areas of high energy nuclear physics require theoretical models to predict the high momenta and binding energies of bound nucleons in the nuclei that describe 2N and 3N SRC in a consistent way. Such 
models are described as nuclear spectral functions that define the joint probability of finding a nucleon in the nucleus with momentum $\mathrm{p}$ and removal (binding) energy $E_{m}$.

The main motivation of the research presented in the present dissertation was therefore to develop a self consistent theoretical model for the calculation of nuclear spectral functions in the domain of the $2 \mathrm{~N}$ and $3 \mathrm{~N}$ short range correlations. For momenta above $400 \mathrm{MeV} / \mathrm{c}$, a relativistic multi-nucleon short range correlation model of the spectral function was obtained. This model describes the high momentum and high missing energy of two and three nucleons in short range correlations ( $2 \mathrm{~N}$ and $3 \mathrm{~N}$ SRC), for symmetric and asymmetric nuclei.

The main assumption for the nuclear spectral function models developed in the present dissertation is the dominance of NN SRC in the nuclear dynamics for internal momenta $\mathrm{p} \gtrsim \mathrm{k}_{\mathrm{src}}>\mathrm{k}_{\mathrm{F}}$, where $\mathrm{k}_{\mathrm{src}}$ is the relative momentum threshold at which a NN system with such relative momentum can be considered in the short-range correlation. The next major assumption is the dominance of the isosinglet $p n$ component in the NN SRC. It was also assumed that the single proton or neutron momentum distributions in the 2N SRC domain are inversely proportional to their relative fractions in nuclei. The inverse proportionality of the high momentum component to the relative fraction of proton or neutron is important for asymmetric nuclei and they need to be included in the modeling of nuclear spectral functions in the $2 \mathrm{~N}$ SRC region. An additional benefit of considering the inverse proportionality is that distinct nuclear spectral function models for neutron and for proton were obtained in the present dissertation.

Considering the dominance of NN SRC described above, a main development in the present dissertation was that the contribution of 3N SRCs to the nuclear spectral function was modeled assuming that such correlations are generated by two sequential $2 \mathrm{~N}$ short range correlations. Hence, the phenomenological knowledge of the properties of 2N SRCs was sufficient to calculate both the $2 \mathrm{~N}$ and $3 \mathrm{~N}$ SRC contributions to the nuclear spectral function. This assumption added to the strong dominance of $p n$ SRCs in nucleus, made it possible to use the momentum distribution of the deuteron as a fundamental component of the developed nuclear spectral function models.

A main focus in the developed models was to treat the relativistic effects which are important for the bound nucleon momenta exceeding characteristic Fermi momentum, $k_{F}$, in 
the nucleus. The relativistic effects in the present dissertation were treated based on the effective Feynman diagrammatic approach, in which the Lorentz covariant amplitudes are reduced to the nuclear spectral functions, in a process of calculation designed to trace the relativistic effects entering in such functions.

One of the main ambiguities related to the treatment of relativistic effects considered in the present dissertation was to account for the vacuum fluctuations ( $Z$ graphs) which ultimately alter the definition of the spectral function as a probability of finding a bound nucleon in the nucleus with the given momentum and removal energy. Two suitable approaches were employed to deal with the relativistic vacuum fluctuations: the virtual nucleon and the lightfront approximations. In the virtual nucleon approximation the vacuum fluctuations were neglected, while in the light-front approximation the vacuum fluctuations are kinematically suppressed.

The amplitudes for the covariant Feynman diagrams for bound nucleons in the mean field of the nucleus, and in 2N and 3N SRCs, were defined by applying the effective Feynman diagrammatic rules. The covariant amplitudes were consistently transformed into nuclear spectral functions to represent bound nucleons in the mean field of the nucleus, and in $2 \mathrm{~N}$ and 3N SRCs. The obtained mean field models are valid for momenta below the Fermi momentum, $\mathrm{k}_{F}$, and were constructed by using a nonrelativistic approach to estimate the ground state wave functions. The models for $2 \mathrm{~N}$ and $3 \mathrm{~N}$ SRCs are valid for momenta above $400 \mathrm{MeV}$. It is therefore expected that the nuclear spectral function models developed in the present dissertation should be valid for the analysis of phenomena important to understand the internal dynamics and structure of a wide range of atomic nuclei.

The computational models for $2 \mathrm{~N}$ and $3 \mathrm{~N}$ SRC high momentum nuclear spectral functions in VN and LF approximations, as well as in the non-relativistic limit of the VN approximation, were obtained in the present dissertation by integration of the delta functions in the mathematical models described above.

The computational models are a set of computable integrals that represent the high momentum nuclear spectral functions, as well as equations for momenta and cosine of angles between momentum vectors, and for auxiliary variables. Restrictions in the values of momenta 
and cosine of angles were also defined to ensure that the results are physically valid. The computational models are ready to be programed in a computer to obtain numerical estimates of nuclear spectral functions, density matrices and momentum distributions.

By applying the developed computational models, the numerical estimates of $2 \mathrm{~N}$ and $3 \mathrm{~N}$ SRC nuclear spectral functions, as well as the numerical estimates and the parametrization of momentum distributions of the bound nucleon, both for $\mathrm{VN}$ and $\mathrm{VN}$ in the nonrelativistic limit approximations, were obtained in the present dissertation.

The predictions for the SRC momentum distribution for proton and neutron for light nuclei $(A \leq 12)$, calculated with the computational models were compared with the momentum distributions from ab initio quantum Monte Carlo calculations. A good agreement, up to \pm 15 $\%$, was found between the predictions and the QMC calculations for momenta in the range 450$1000 \mathrm{MeV} / \mathrm{c}$, both for proton and neutron. The results showed that the SRC high momentum nuclear spectral function models developed in the present dissertation, describe reasonably well the high momentum spectral function and the high momentum distributions for the light nuclei in the nonrelativistic limit of the VN approximation.

The mean-field and SRC momentum distributions in VNA and VN in the nonrelativistic limit were parametrized for a wide range of nuclei $(3 \leq A \leq 208)$, resulting in a set of fitting parameters useful for further calculations.

\subsection{Application of the multi-nucleon short range correlation model for the nuclear spectral functions}

Some of the experiments for which the multi-nucleon short range correlation model for the nuclear spectral functions developed in the present dissertation may be applied for their analysis are the following.

\subsubsection{Experiments at Jefferson Lab Hall A}

- E08-14: $x>2$ Short range correlations: Three-nucleon short range correlations studies in Inclusive scattering for $0.8<Q^{2}<2.8(\mathrm{Gev} / \mathrm{c})^{2}$ 
- E07-006 : Short Range Correlation: Studying short-range correlations in nuclei at the repulsive core limit via the triple coincidence $\left(e, e^{\prime} p N\right)$ reaction.

- E01-015: Short Range Correlation Experiment: Studying the internal small distance structure of nuclei via the triple coincidence $\left(e, e^{\prime} p+N\right)$ measurement.

- E97-111 : Systematic Probe of Short-Range Correlations via 4He(e,e'p)3He: The experiment consists of making measurements of the cross section for the reaction $4 \mathrm{He}\left(\mathrm{e}, \mathrm{e}^{\prime} \mathrm{p}\right) 3 \mathrm{H}$.

- E89-004 : Selected studies of the ${ }^{3} \mathrm{He}$ and ${ }^{4} \mathrm{He}$ Nuclei Through Electrodisintegration at High Momentum Transfer

\subsubsection{Experiments at the BooNE collaboration}

The experiments of interest at the BooNE (Booster Neutrino Experiment) collaboration are the neutrino-nucleus scattering which has the goal of understanding neutrino oscillations. The experiments are described in reference [95] .

\subsubsection{Experiments at the NUTEV collaboration}

The experiments of interest at the NUTEV collaboration are the neutrino-nuclei scatterings on which a discrepancy have been found between measurements of $\sin ^{2} \theta_{w}\left(\theta_{w}\right.$ is the weak mixing angle) involving free particles and those involving bound nucleons in the nuclear medium, the so-called NuTev anomaly $[73,77,111]$. A possible explanation to the experimental results is the presence of more energetics protons in neutron rich, large A, asymmetric nuclei which implies that $\mathrm{u}$-quarks are more modified than d-quarks, resulting in a negative correction for the experimental value of $\sin ^{2} \theta_{W}$ for bound nucleons [111]. The application of the models of the nuclear spectra functions may increase the understanding of the medium modification of the parton distribution functions (PDFs) as well as the realistic treatment of SRCs involved in such experiments. 


\section{Bibliography}

[1] E. Rutherford, "The Scattering of $\alpha$ and $\beta$ Particles by Matter and the Structure of Atoms", Phil. Mag. 21, 669 (1911).

[2] E. Rutherford, "Collision of $\alpha$ Particles With Light Atoms, part IV. An Anomalous Effect in Nitrogen", Phil. Mag. 37, 581 (1919).

[3] E. Rutherford, "Bakerian Lecture: Nuclear Constitution of Atoms", Proc. Roy. Soc. A97, 374(1920).

[4] N. F. Mott, "The Scattering of Fast Electrons by Atomic Nuclei", Proc. Roy. Soc. A 124, 425(1929).

[5] J. Chadwick, "The Existence of a Neutron", Proc. Roy. Soc. A 136, 692 (1932).

[6] I. Curie-Joliot and F. Joliot-Curie, "The Emission of High energy Photons from Hydrogenous Substances Irradiated with Very Penetrating Alpha Rays", Compt. Rend. 194, 273 (1932).

[7] W. Heisenberg, "On the Structure of Atomic Nuclei. I. ", Z.Phys. 77,1 (1932).

[8] H. Yukawa, "On the Interaction of Elementary Particles", Proc. Phys. Math. Soc. Japan 17, 48 (1935).

[9] C.M.G. Lates, H. Muirhead, G.P.S. Occhialini, and C.F. Powell, "Processes Involving Charged Mesons", Nature 159, 694 (1947).

[10] P.A.M. Dirac, "Forms of Relativistic Dynamics", Rev. Mod. Phys. 21, 392 (1949).

[11] M.Rosenbluth, "High Energy Elastic Scattering of Electrons on Protons", Phys. Rev. 79, 615 (1950).

[12] E.E. Salpeter, and H.A. Bethe , "A Relativistic Equation for Bound-State Problems", Phys. Rev. 84, 1232 (1951).

[13] C.N. Yang and R.L. Mills, "Conservation of Isotopic Spin and Isotopic Gauge Invariance", Phys. Rev. 96, 191 (1954).

[14] M.G. Mayer and J.H.D. Jensen, Elementary Theory of Nuclear Shell Structure, (John Wiley and Sons, New York, 1955).

[15] R. Hofstadter, F. Bumiller, and M.R. Yearian, "Electromagnetic Structure of the Proton and Neutron", Rev. Mod. Phys. 30, 482 (1958).

[16] M. Gell-Mann, The Eightfold Way: A Theory of Strong Interaction Symmetry, (Washington DC, U.S. Dept. of Energy, 1961).

[17] Y. Ne'eman, "Derivation of strong interactions from a gauge invariance", Nuc. Phys. 26, $222(1961)$. 
[18] L.N. Hand, D.G. Miller, and R. Wilson, "Electric and Magnetic Form Factors of the Nucleon", Rev. Mod. Phys. 35, 335 (1963).

[19] A. de-Shalit and I. Talmi, Nuclear Shell Theory, ( Academic Press, New York, 1963).

[20] M. Gell-Mann, "A Schematic Model of Baryons and Mesons", Phys. Lett. 8, 214 (1964).

[21] G. Zweig, "An SU3 model for strong interaction symmetry and its breaking", Version 1, CERN-8182/Th.401 (Jan.1964), unpublished.

[22] G. Zweig, "An SU3 model for strong interaction symmetry and its breaking", Version 2, CERN-8182/Th.412 (Feb.1964), unpublished.

[23] S.D. Drell, and J.D. Walecka, "Electrodynamic processes with nuclear targets", Ann.Phys. 28, 18 (1964).

[24] N.F. Mott and H.S.W. Massey, The Theory of Atomic Collisions, (Oxford University Press, New York, 1965).

[25] S. Weinberg, "Dynamics at infinite momentum", Phys. Rev. 150, 1313 (1966).

[26] J.D. Bjorken and E.A. Paschos, "Inelastic Electron-Proton and $\gamma$ Proton Scattering and the Structure of the Nucleon", Phys. Rev. 185, 1975 (1969).

[27] V. N. Gribov, "Interaction of gamma quanta and electrons with nuclei at high-energies", Sov. Phys. JETP 30, 709 (1970).

[28] E. J. Moniz, I. Sick, R. R. Whitney, J. R. Ficenec, R. D. Kephart, and W. P. Trower "Nuclear Fermi Momenta from Quasielastic Electron Scattering", Phys. Rev. Lett. 26, 445 (1971).

[29] R. Feynman, Photon-Hadron Interactions, ( Addisson-Wesley,Massachusetts, 1972).

[30] L. Bertocchi, "Graphs and glauber", Nuovo Cimento A 11, 45 (1972).

[31] H. Fritzsch, M. Gell-Mann, and H. Leutwyler, "Advantages of the color octet gluon picture", Phys. Lett. B 47, 365 (1973).

[32] F. Gross, "New theory of nuclear forces. Relativistic origin of the repulsive core", Phys. Rev. D 10,223 (1974).

[33] M.A. Preston and R.K. Bhaduri, Structure of the Nucleus, (Addison-Wesley Publishing Company, Reading, 1975).

[34] F. E. Close, An Introduction to Quarks and Partons, (Academic Press,London, 1979).

[35] A. Bodek et al., "Experimental studies of the neutron and proton electromagnetic structure functions", Phys. Rev. D 20, 1471 (1979).

[36] L. L. Frankfurt and M. I. Strikman, "High-Energy Phenomena, Short Range Nuclear Structure and QCD," Phys. Rept. 76, 215 (1981).

[37] F. Gross, "The Relativistic Few Body Problem. 1. Two-Body Equations," Phys. Rev. C 26, 2203 (1982). 
[38] J. J. Aubert et. al., "The ratio of the nucleon structure functions $F_{2}^{N}$ for iron and deuterium", Phys. Lett. B 123, 275 (1983).

[39] Taber De Forest, "Off-shell electron-nucleon cross sections: The impulse approximation", Nucl. Phys. A 392, 232 (1983).

[40] M. Hanzel and A. D. Martin, Quarks and Leptons: An Introductory Course in Modern Particle Physics, (John Wiley and Sons, New York, 1984).

[41] L. L. Frankfurt and M. I. Strikman, "On the Normalization of Nucleus Spectral Function and the EMC Effect", Phys. Lett. B 183, 254 (1987).

[42] J. Ashman et. al., (European Muon Collaboration), "Measurement of the ratios of deep inelastic muon-nucleus cross sections on various nuclei compared to deuterium", Phys. Lett. B 202, 603 (1988).

[43] L. L. Frankfurt and M. I. Strikman, "Hard Nuclear Processes and Microscopic Nuclear Structure", Phys. Rept. 160, 235 (1988).

[44] R.B. Wiringa, "Variational calculations of few-body nuclei", Phys. Rev. C 43,1585 (1991).

[45] C. Ciofi degli Atti, S. Simula, L. L. Frankfurt and M. I. Strikman, ”Two nucleon correlations and the structure of the nucleon spectral function at high values of momentum and removal energy", Phys. Rev. C 44, R7(R) (1991).

[46] L. Frankfurt and M. Strikman, "Short range correlations in nuclei as seen in hard nuclear reactions and light cone dynamics", in Modern topics in electron scattering, edited by B. Frois and I. Sick (World Scientific, Singapore, 1991), pp. 645-694.

[47] C. Ciofi degli Atti, B.D. Day, and S. Liuti, "Quasielastic and inelastic inclusive electron scattering by nuclear systems: Nucleon momentum distributions, spectral functions, and offshell effects", Phys. Rev. C 46, 1045 (1992).

[48] F. Gross, J. W. Van Orden and K. Holinde, "Relativistic one boson exchange model for the nucleon-nucleon interaction", Phys. Rev. C 45, 2094 (1992).

[49] L. L. Frankfurt, M. I. Strikman, D. B. Day and M. Sargsian, "Evidence for short range correlations from high $\mathrm{Q}^{2}$ (e, e-prime) reactions", Phys. Rev. C 48, 2451 (1993)

[50] I. Talmi, Simple Models of Complex Nuclei, (Hardwood Academic Publishers GmbH, Switzerland, 1993).

[51] R.B. Wiringa, V.G.J. Stoks and R.Schiavilla, "Accurate nucleon-nucleon potential with charge-independence breaking", Phys. Rev. C 51, 38 (1995).

[52] C. Ciofi degli Atti and S. Simula, "Realistic model of the nucleon spectral function in few and many nucleon systems", Phys. Rev. C 53, 1689 (1996).

[53] L. L. Frankfurt, M. M. Sargsian and M. I. Strikman, "Feynman graphs and generalized eikonal approach to high-energy knockout processes", Phys. Rev. C 56, 1124 (1997) 
[54] L.M. Brown, M. Riordan, M. Dresden, and L. Hodddeson in: The Rise of the Standard Model: Particle Physics in the 1960s and 1970s, eds. L.M. Brown, M. Riordan, M. Dresden, and L. Hodddeson, (Cambridge University Press, Cambridge, 1997) p3.

[55] N. Samios in: The Rise of the Standard Model: Particle Physics in the 1960s and 1970s, eds. L.M. Brown, M. Riordan, M. Dresden, and L. Hodddeson, (Cambridge University Press, Cambridge, 1997) p525.

[56] J. Friedman in: The Rise of the Standard Model: Particle Physics in the 1960s and 1970s, eds. L.M. Brown, M. Riordan, M. Dresden, and L. Hodddeson, (Cambridge University Press, Cambridge, 1997) p566.

[57] J.Bjorken in: The Rise of the Standard Model: Particle Physics in the 1960s and 1970s, eds. L.M. Brown, M. Riordan, M. Dresden, and L. Hodddeson, (Cambridge University Press, Cambridge, 1997) p589.

[58] G.A. Miller, "Light front treatment of nuclei: Formalism and simple applications", Phys. Rev. C 56, 2789 (1997).

[59] B. S. Pudliner, V. R. Pandharipande, J.Carlson, S.C. Pieper, and R. B. Wiringa, "Quantum Monte Carlo calculations of nuclei with $A<7$ ", Phys. Rev. C 56, 1720(1997).

[60] M.S. Fayache, L. Zamick, and B. Castel, "The nuclear tensor interaction", Phys. Rep. 290, 201 (1997).

[61] L. Frankfurt, M. Strikman, G. Piller and M. Sargsian, "Feynman diagram approach to highenergy scattering from lightest nuclei”, Nucl. Phys. A 631, 502 (1998).

[62] S. J. Brodsky, H. C. Pauli and S. S. Pinsky, "Quantum chromodynamics and other field theories on the light cone", Phys. Rept. 301, 299 (1998).

[63] A. Harindranath, "An Introduction to Light-Front Dynamics for Pedestrians", arXiv:hepph: 9612244v2 (1998).

[64] J. L. S. Aclander et. al., "The large momentum transfer reaction $C-12(p, 2 p+n)$ as a new method for measuring short range NN correlations in nuclei”, Phys. Lett. B 453, 211 (1999).

[65] R. B. Wiringa , S.C. Pieper, J.Carlson, and V. R. Pandharipande, "Quantum Monte Carlo calculations of A = 8 nuclei ", Phys. Rev. C 62, 014001 (2000).

[66] G. A. Miller, "Light front quantization: A Technique for relativistic and realistic nuclear physics", Prog. Part. Nucl. Phys. 45, 83 (2000)

[67] S. C. Pieper and R. B. Wiringa, "Quantum Monte Carlo Calculations of Light Nuclei", Ann. Rev. Nucl. Part. Sci. 51, 53 (2001).

[68] M. M. Sargsian, "Selected topics in high energy semiexclusive electronuclear reactions", Int. J. Mod. Phys. E 10, 405 (2001)

[69] R. B. Wiringa, http://www.phy.anl.gov/theory/research/momenta/. 
[70] M.M. Sargsian, S. Simula, and M.I. Strikman, "Neutron structure function and inclusive deep inelastic scattering from ${ }^{3} \mathrm{H}$ and ${ }^{3} \mathrm{He}$ at large Bjorken $x$ ", Phys. Rev. C 66, 024001 (2002).

[71] S.C. Pieper, K.Varga, and R. B. Wiringa, "Quantum Monte Carlo calculations of $A=9,10$ nuclei", Phys. Rev. C 66, 044310 (2002).

[72] The LEP collaborators, "A Combination of Preliminary Electroweak Measurements and Constraints on the Standard Model", Report No. CERN-EP/2001-98, arXiv:hepex:0112021v2 (2002).

[73] G.P.Zeller et. al. (NuTeVCollaboration), "Precise Determination of Electroweak Parameters in Neutrino-Nucleon Scattering", Phys. Rev. Lett. 88, 091802 (2002).

[74] I. Yaron, J. Alster, L. Frankfurt, E. Piasetzky, M. Sargsian and M. Strikman, "Investigation of the high momentum component of nuclear wave function using hard quasielastic $A(p, 2 p) X$ reactions", Phys. Rev. C 66, 024601 (2002).

[75] M. M. Sargsian et. al., "Hadrons in the Nuclear Medium”, J. Phys. Phys. G 29, R1 (2003)

[76] P. R. Norton, ”The EMC effect”, Rep. Prog. Phys. 66, 1253 (2003)

[77] G. P. Zeller et. al. (NuTeVCollaboration), "A Precise determination of electroweak parameters in neutrino nucleon scattering", Phys. Rev. Lett. 90, 239902 (2003)

[78] K. S. Egiyan et. al. (CLAS Collaboration), "Observation of nuclear scaling in the A(e, eprime) reaction at x(B) greater than 1", Phys. Rev. C 68, 014313 (2003).

[79] A. Tang et. al., " $n-p$ Short-Range Correlations from $(p, 2 p+n)$, Phys Rev. Lett. 90, 042301 (2003).

[80] P. Ring and P. Schuck, The Nuclear Many Body Problem ( Springer-Verlag, New York, 2004).

[81] M. M. Sargsian, T. V. Abrahamyan, M. I. Strikman and L. L. Frankfurt, "Exclusive electrodisintegration of He-3 at high $\mathrm{Q}^{2}$. I. Generalized eikonal approximation”, Phys. Rev. C 71, 044614 (2005).

[82] M. M. Sargsian, T. V. Abrahamyan, M. I. Strikman and L. L. Frankfurt, ”Exclusive electrodisintegration of He-3 at high Q2 ${ }^{2}$ II. Decay function formalism”, Phys. Rev. C 71, 044615 (2005).

[83] G.B. Arfken and H.J. Weber, Mathematical Methods for Physicists, Sixth edition (Elsevier Academic Press, Burlington, 2005).

[84] K. S. Egiyan et. al. (CLAS Collaboration), "Measurement of 2- and 3-nucleon short range correlation probabilities in nuclei", Phys. Rev. Lett. 96, 082501 (2006).

[85] E. Piasetzky, M. Sargsian, L. Frankfurt, M. Strikman and J. W. Watson, "Evidence for the strong dominance of proton-neutron correlations in nuclei”, Phys. Rev. Lett. 97, 162504 (2006).

[86] R. Schiavilla, R. B. Wiringa, S. C. Pieper and J. Carlson, "Tensor Forces and the GroundState Structure of Nuclei”, Phys. Rev. Lett. 98, 132501 (2007). 
[87] R. Shneor et al. (Jefferson Lab Hall A Collaboration) "Investigation of Proton-Proton ShortRange Correlations via the C12(e,e?pp) Reaction", Phys. Rev. Lett. 99, 072501 (2007).

[88] L. Frankfurt, M. Sargsian and M. Strikman, "Recent observation of short range nucleon correlations in nuclei and their implications for the structure of nuclei and neutron stars", Int. J. Mod. Phys. A 23, 2991 (2008).

[89] L. Frankfurt, M. Sargsian M. Strikman, "Future directions for probing two and three nucleon short-range correlations at high energies", AIP.Conf.Proc.1056, 322 (2008).

[90] R. Subedi, R. Shneor, P. Monaghan, B. D. Anderson, K. Aniol, J. Annand, J. Arrington and H. Benaoum et al., "Probing Cold Dense Nuclear Matter", Science 320, 1476 (2008)

[91] J. Seely et. al., "New Measurements of the European Muon Collaboration Effect in Very Light Nuclei", Phys. Rev. Lett. 103, 202301 (2009).

[92] D.V. Higinbotham, J.Gomez and E. PiasetzKy, "Nuclear Scaling and the EMC Effect", arXiv:hep-ph: 1003.4497v2 (2010).

[93] M. M. Sargsian,"Large $Q^{2}$ Electrodisintegration of the Deuteron in Virtual Nucleon Approximation", Phys. Rev. C 82, 014612 (2010)

[94] M.M. Sargsian, private communication, 2011.

[95] R. Tayloe, Indiana U. APS-DPF 2011 Providence, RI, 8/11, http://www-boone.fnal.gov

[96] W. U. Boeglin et. al. [Hall A Collaboration], "Probing the high momentum component of the deuteron at high $\mathrm{Q}^{2}$ ”, Phys. Rev. Lett. 107, 262501 (2011)

[97] L.B. Weinstein, E. Piasetzhy, D.W. Higinbotham, J.Gomez, O. Hen, and R. Shneor, "Short Range Correlations and the EMC Effect”, Phys. Rev. Lett. 106, 052301 (2011).

[98] W. Cosyn and M. Sargsian, "Final-state interactions in semi-inclusive deep inelastic scattering off the Deuteron", Phys. Rev. C 84, 014601 (2011).

[99] J. Arrington, D.V. Higinbotham, G. Rosner, and M. Sargsian, "Hard probes of short-range nucleon-nucleon correlations", Prog. Part. Nucl. Phys.67,898 (2012).

[100] O. Hen, E. Piasetzky and L. B. Weinstein, "New data strengthen the connection between Short Range Correlations and the EMC effect", Phys. Rev. C 85, 047301 (2012)

[101] S.J. Beringer et. al. (Particle Data Group, Structure Functions), Phys. Rev. D6, 010001(2012)

[102] S.J. Beringer et. al. (Particle Data Group, Electro-weak model) , Phys. Rev. D6, 010001(2012).

[103] N. Fomin, J. Arrington, R. Asaturyan, F. Benmokhtar, W. Boeglin, P. Bosted, A. Bruell and M. H. S. Bukhari, "New measurements of high-momentum nucleons and short-range structures in nuclei", Phys. Rev. Lett. 108, 092502 (2012).

[104] M. McGauley and M. M. Sargsian, ”Off-Fermi Shell Nucleons in Superdense Asymmetric Nuclear Matter", arXiv:1102.3973v3 (2012). 
[105] M. Vanhalst, J. Ryckebusch and W. Cosyn, "Quantifying short-range correlations in nuclei”, Phys. Rev. C 86, 044619 (2012).

[106] J. Arrington, D. W. Higinbotham, G. Rosner and M. Sargsian, "Hard probes of short-range nucleon-nucleon correlations", Prog. Part. Nucl. Phys. 67, 898 (2012).

[107] M. Sargsian, "The EMC effect and short-range correlation”, AIP Conf.Proc. 1560, 480 (2013).

[108] O. Hen, D. W. Higinbotham, G. A.Miller, E. Piasetzhy and L. B. Weinstein "The EMC effect and high momentum nucleons in nuclei”, Int. J. Mod. Phys. E 22, 1330017 (2013)

[109] M. Alvioli, C. Ciofi degli Atti, L. P. Kaptari, C. B. Mezzetti and H. Morita, "Nucleon momentum distributions, their spin-isospin dependence and short-range correlations", Phys. Rev. C 87, 034603 (2013).

[110] M. Alvioli, C. Ciofi Degli Atti, L. P. Kaptari, C. B. Mezzetti and H. Morita, "Universality of nucleon-nucleon short-range correlations and nucleon momentum distributions", Int. J. Mod. Phys. E 22, 1330021 (2013).

[111] M. Sargsian, "Neutrino interactions in the nuclear environment", APS April Meeting 2013, April 13-16, 2013 (unpublished)

[112] M. M. Sargsian, "New properties of the high-momentum distribution of nucleons in asymmetric nuclei”, Phys. Rev. C 89, no. 3, 034305 (2014).

[113] W. Cosyn, W. Melnitchouk and M. M. Sargsian, "Final-state interactions in inclusive deep-inelastic scattering from the deuteron”, Phys. Rev. C 89, 014612 (2014).

[114] M. M. Sargsian, "Nucleon-Nucleon Interactions at Short Distances", arXiv:nuclth:1403.0678v1 (2014).

[115] W. U. Boeglin et. al., "Deuteron Electro-Disintegration at Very High Missing Momenta", arXiv:nucl-ex:1410.6770v1 (2014).

[116] A. Rios, A. Polls and W. H. Dickhoff, "Density and isospin asymmetry dependence of highmomentum components", Phys. Rev. C 89, 044303 (2014).

[117] S.J. Beringer et. al., (Particle Data Group, Quantum Chromodynamics) Chin. Phys. C,86, 090001(2014).

[118] G. R. Jansen, J. Engel, G. Hagen, P. Navratil and A. Signoracci, "Ab-initio coupled-cluster effective interactions for the shell model: Application to neutron-rich oxygen and carbon isotopes", Phys. Rev. Lett. 113, 142502 (2014).

[119] O. Hen, M. Sargsian, L. B. Weinstein, E. Piasetzky, H. Hakobyan, D. W. Higinbotham, M. Braverman and W. K. Brooks, "Momentum sharing in imbalanced Fermi systems", et al.,Science 346, 614 (2014)

[120] R. B. Wiringa, R. Schiavilla, S. C. Pieper and J. Carlson, "Nucleon and nucleon-pair momentum distributions in $A \leq 12$ nuclei", Phys. Rev. C 89, no. 2, 024305 (2014). 
[121] I. Korover et al. (Jefferson Lab Hall A Collaboration), "Probing the Repulsive Core of the Nucleon-Nucleon Interaction via the He4(e,e?pN) Triple-Coincidence Reaction", Phys. Rev. Lett. 113, 022501 (2014).

[122] C. Colle, W. Cosyn, J. Ryckebusch and M. Vanhalst, "Factorization of exclusive electroninduced two-nucleon knockout", Phys. Rev. C 89, 024603 (2014).

[123] M. Sargsian, "Zverev mean field wave function", private communication.

[124] J. Ryckebusch, W. Cosyn and M. Vanhalst, "Stylized features of single-nucleon momentum distributions", J. Phys. G 42, 055104 (2015).

[125] A. J. Freese, M. M. Sargsian and M. I. Strikman, "Probing superfast quarks in nuclei through dijet production at the LHC”, Eur. Phys. J. C 75, 534 (2015).

[126] A. J. Freese and M. M. Sargsian, "QCD Evolution of Superfast Quarks", arXiv:1511.06044v1 (2015).

[127] W. Boeglin and M. Sargsian, "Modern Studies of the Deuteron: from the Lab Frame to the Light Front", Int. J. Mod. Phys. E 24, no. 03, 1530003 (2015)

[128] C. Ciofi degli Atti, "'In-medium short-range dynamics of nucleons: Recent theoretical and experimental advances", Phys. Rept. 590, 1 (2015).

[129] T. Neff, H. Feldmeier and W. Horiuchi, "Short-range correlations in nuclei with similarity renormalization group transformations", Phys. Rev. C 92, 024003 (2015)

[130] C. Colle, O. Hen, W. Cosyn, I. Korover, E. Piasetzky, J. Ryckebusch and L. B. Weinstein, "Extracting the mass dependence and quantum numbers of short-range correlated pairs from $A(e, e p)$ and $A(e, e p p)$ scattering", Phys. Rev. C 92, 024604 (2015).

[131] O. Hen, L. B. Weinstein, E. Piasetzky, G. A. Miller, M. M. Sargsian and Y. Sagi, ”Correlated fermions in nuclei and ultracold atomic gases", Phys. Rev. C 92, 045205 (2015).

[132] S. Scopetta, A. Del Dotto, L. Kaptari, E. Pace, M. Rinaldi and G. Salm, ”A Light-Front Approach to the ${ }^{3}$ He Spectral Function”, Few Body Syst. 56, 425 (2015).

[133] D. W. Higinbotham and O. Hen, "Comment on Measurement of Two- and Three-Nucleon Short-Range Correlation Probabilities in Nuclei”, Phys. Rev. Lett. 114, 169201 (2015). 
VITA

\title{
OSWALDO ARTILES
}

\author{
1964-1970 \\ Electrical Engineering \\ Universidad Central de Venezuela \\ Caracas, Venezuela \\ 1976-1978 \\ M.Sc., Power Systems Analysis \\ The University of Manchester, \\ Institute of Science and Technology (UMIST), \\ Manchester, England \\ 1996-1997 \\ Specialist in Finance \\ Instituto de Estudios Superiores de Administración (IESA), \\ Caracas, Venezuela \\ $1972-1999$ \\ Electrical Engineer and Manager \\ C.V.G. Electrificación del Caroni (EDELCA) \\ Caracas, Venezuela \\ $1999-2003$ \\ President \\ C.V.G. Electrificación del Caroni (EDELCA) \\ Caracas, Venezuela \\ $2005-2007$ \\ B.Sc., Physics \\ University of Miami \\ Miami, Florida, USA. \\ $2009-2012$ \\ M.Sc., Physics \\ Florida International University \\ Miami, Florida, USA. \\ $2012-2017$ \\ Ph.D., Physics \\ Florida International University \\ Miami, Florida,USA. \\ 1979 - present \\ Life Senior Member of the Institute of Electrical and Electronic \\ Engineers (IEEE) \\ 2012 - present \\ Member of the American Physical Society (APS)
}




\section{SELECTED PUBLICATIONS AND PRESENTATIONS}

1. Oswaldo Artiles and Misak Sargsian, Multinucleon short-range correlation model for nuclear spectral functions: Theoretical framework, Physical Review C88, 044604 (2013)

2. Oswaldo Artiles and Misak Sargsian, Multinucleon short-range correlation model for nuclear spectral functions: Numerical estimates, in preparation

3. Oswaldo Artiles, Multinucleon short-range correlation model for nuclear spectral functions, talk presented at APS April meeting, Washington,DC, January 2015. 